\title{
ZnO thin-film optimization towards the fabrication of \\ high-frequency ultrasound transducers
}

by

Ihab Sinno

\begin{abstract}
A thesis
submitted to the Victoria University of Wellington

in fulfilment of the requirements for the degree of

Doctor of Philosophy
\end{abstract}

Victoria University of Wellington

2017 


\section{Abstract}

Zinc oxide is a popular wide bandgap semiconductor material with versatile electrical and optical properties. In its wurtzite crystal form, this semiconductor is piezoelectric, and has material properties that make it an attractive candidate for fabricating high frequency ultrasound transducers. This thesis describes the development of an RF sputtering process for creating zinc oxide films with thicknesses ranging from $3 \mu \mathrm{m}$ to $10 \mu \mathrm{m}$, aiming for transducer frequencies of $300 \mathrm{MHz}$ to $1 \mathrm{GHz}$. Sputtering parameters are optimized to meet the dual requirements of a c-axis film orientation while maintaining a high deposition rate. These constraints and the dimensional characteristics of the utilized sputtering system, such as the short substrate-to-target distance, introduce high levels of strain in the deposited zinc oxide films. Various anneal procedures are developed to reduce film strain and optimize the resulting microstructure. It is found that annealing temperatures $>600^{\circ} \mathrm{C}$ eliminate the inherent film strain, but simultaneously result in the dewetting of the bottom metal contact, making this thermal treatment unsuitable for device processing. As an alternative to traditional metal contacts used in ultrasound transducers, the use of highly doped zinc oxide contacts is then investigated. It is shown that aluminium doped zinc oxide contacts provide an improved seed layer for device growth while eliminating the dewetting problems associated with metal contacts at high anneal temperatures. In addition, the use of such transparent conductive oxide contacts can lead to novel ultrasound applications, which benefit from the integration of optical and acoustic imaging in a single lens. A proof of concept all-zinc oxide single element ultrasound transducer 
structure is finally fabricated, to highlight the potential of an integrated opticalacoustic lens design. 
This page is intentionally left blank 


\section{Acknowledgements}

I would first like to thank my supervisors Gideon Gouws and Ciaran Moore for the invaluable advice over the course of my PhD. I greatly appreciate the effort, time, and resources you have put into supporting my research, and the feedback on the work carried throughout. Your patience, insight, and positive mindsets were crucial for the success of this work.

I am grateful to Callaghan Innovation and Victoria University of Wellington for awarding me the PhD, FSRG, and submission Scholarships, and to my supervisors for supporting these applications. Without the financial support, this research would not be possible.

I would also like to thank the technical staff at VUW, Paul Harris at Callaghan Innovation, and Sarah Spencer at the Robinson Research Institute for their valuable support. To my friends with whom I have had the pleasure of sharing life with during these quick years, thank you for your kind gestures, interesting stories, and tolerating my long-winded discussions and complaints. Thank you in particular to Ahmad Al-Imam, Mohsen Sichani, Hanieh Ghominejad, Ahmad Sheikh Deeb, Eden Rafaelov, Brett Ryan, Caleb Keene, Diana Siwiak, Xu He, Farzad Radmehr, Mozhgan Mohammadpour, Diego Navarro, Conor Burke-Govey, Mohammad Zarei, and Zakwan Al-Arnaout.

Last but not least, I give thanks to my parents and family for supporting what I stand for. Your sacrifice and unconditional love are driving forces to my existence. I 
love you. To my better half, Farzaneh Fadakar Masouleh, your love, kindness, support, and friendship were my one-way ticket to happily ever after. Az hameh bishtar dooset daram Shabijunam ;) 


\section{Contents}

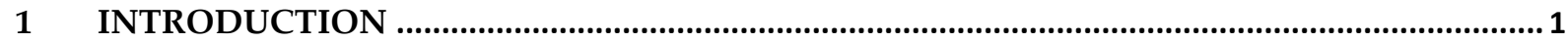

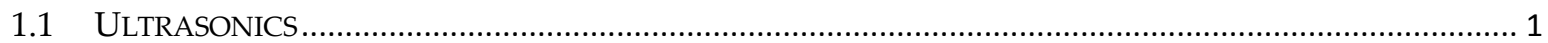

1.2 Ultrasound TRANSDUCERS AND ACOUSTIC MicrosCopy CONSIDERATIONS..................................... 3

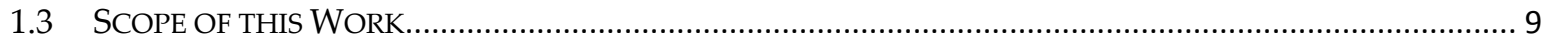

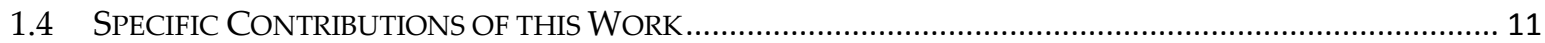

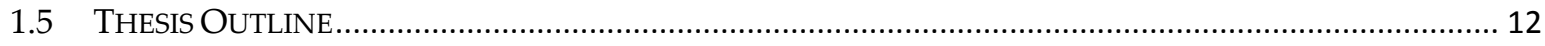

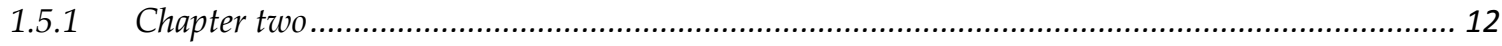

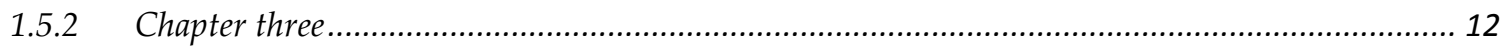

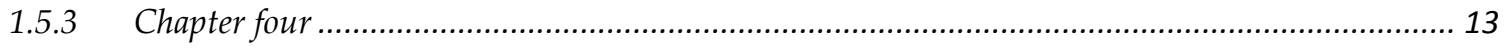

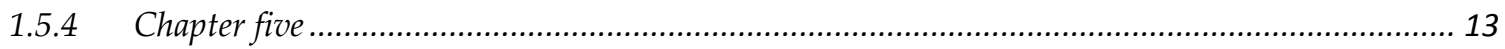

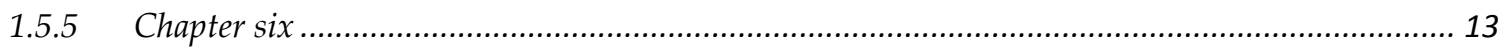

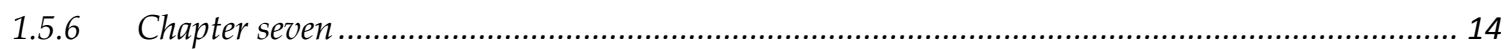

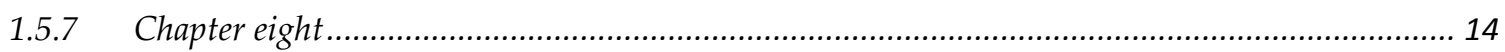

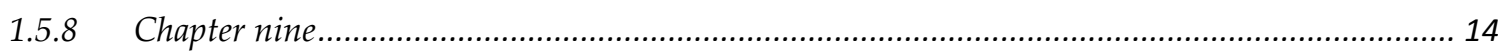

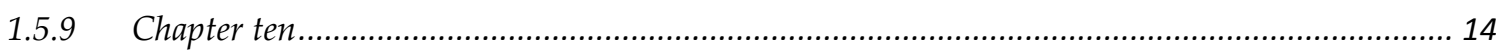

2 ZNO AS THE PIEZOELECTRIC MATERIAL …..................................................................

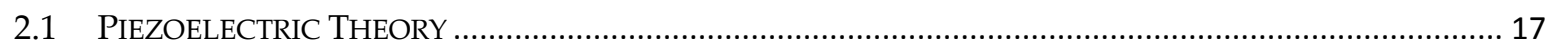

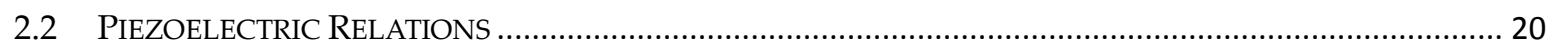

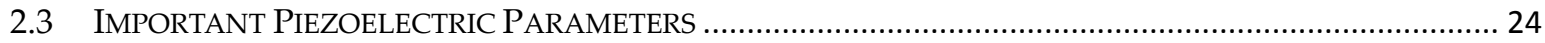

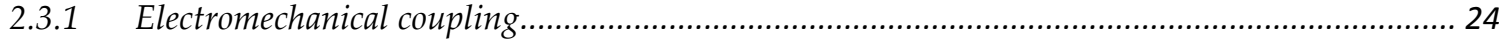

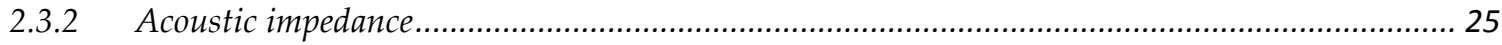

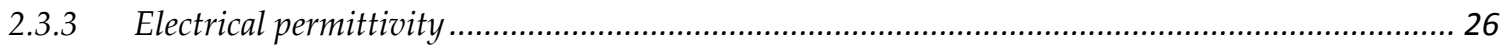

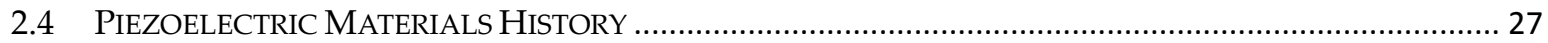

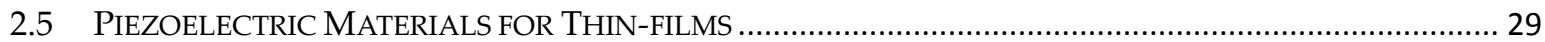

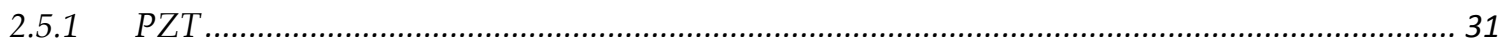


2.5.2 AIN …

2.5.3 $\mathrm{ZnO}$

2.6 StATUS QUO OF High-FREQUENCY UlTRASOUND TRANSDUCERS ...................................................... 43

3 FABRICATION

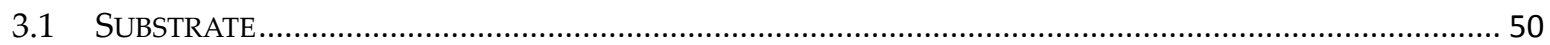

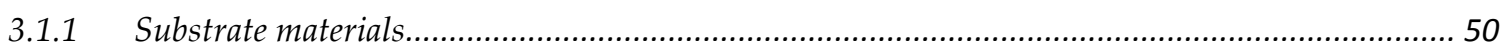

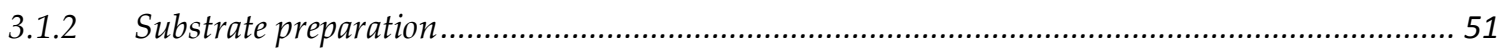

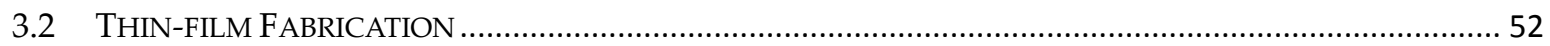

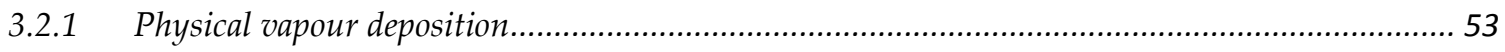

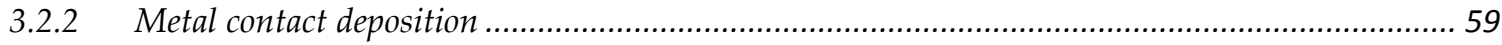

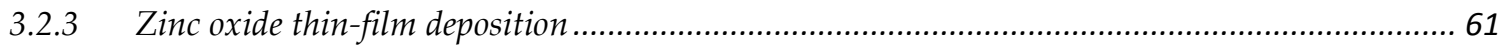

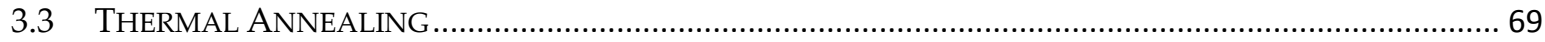

$4 \quad$ CHARACTERIZATION TECHNIQUES ............................................................................................. 71

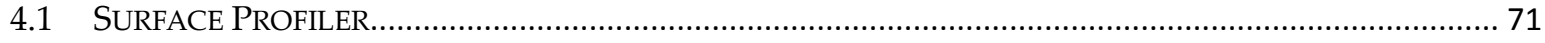

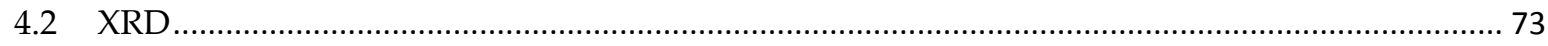

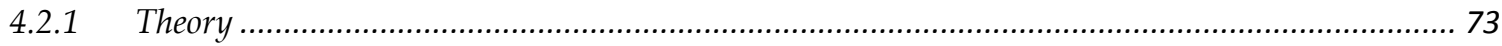

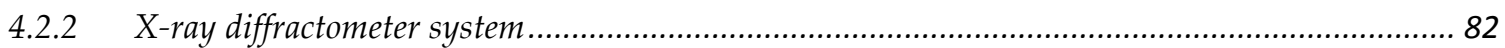

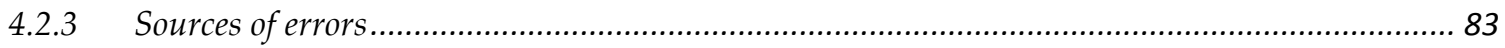

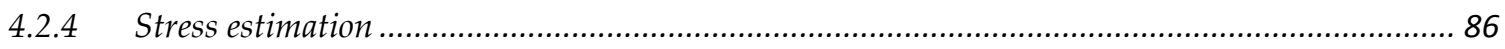

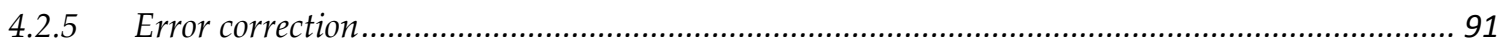

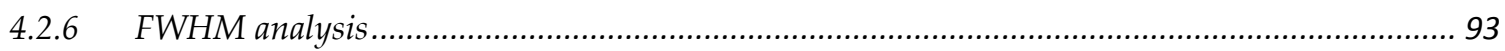

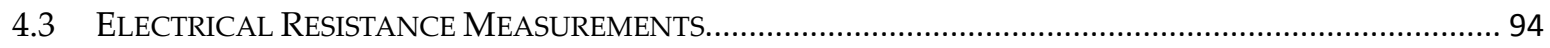

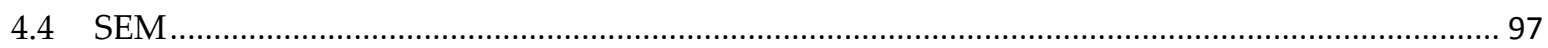

4.4.1 Imaging using the secondary electron detector ............................................................... 97

4.4.2 Imaging using the backscattered electron detector ......................................................... 98

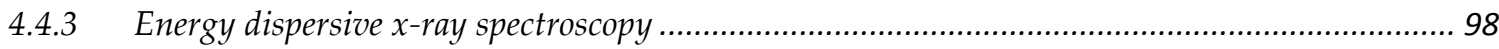

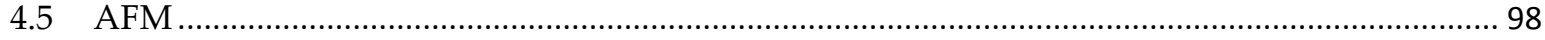

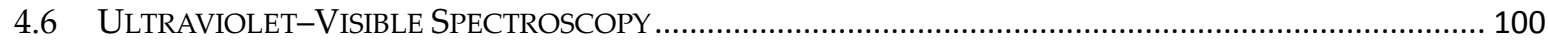

$5 \quad$ RESULTS A: SPUTTERING PARAMETER OPTIMIZATION ...................................................107

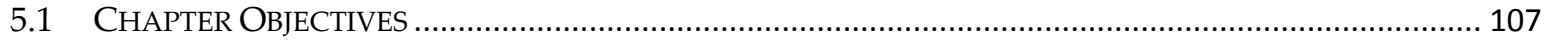

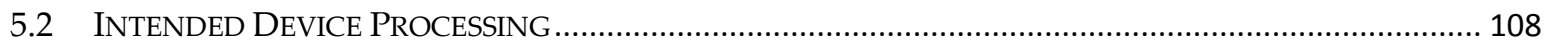




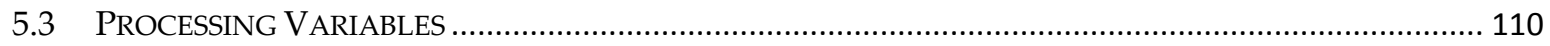

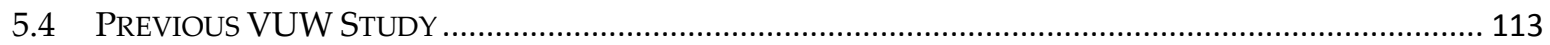

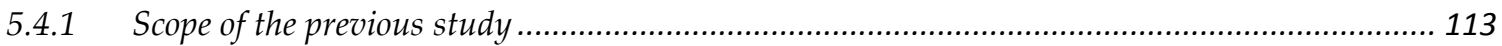

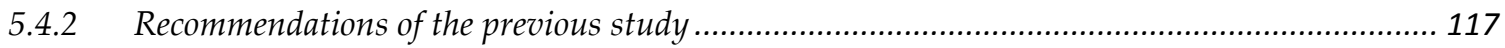

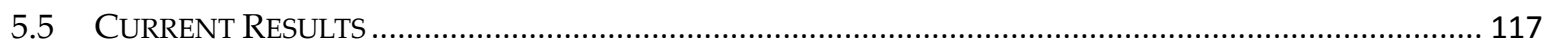

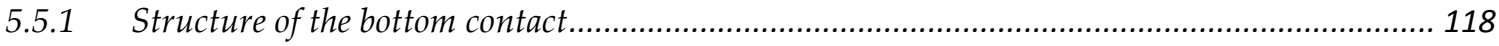

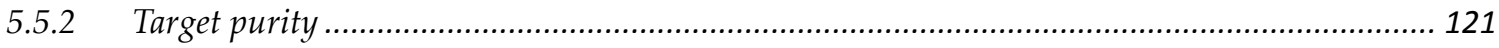

5.5.3 Substrate-target placement and thickness uniformity .......................................................... 122

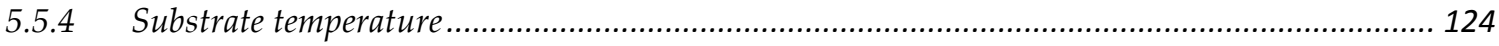

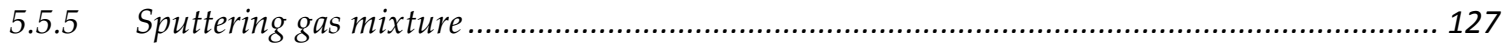

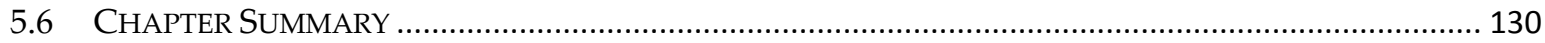

6 RESULTS B: THERMAL ANNEALING EFFECTS ON THE ZNO FILMS' STRUCTURAL AND

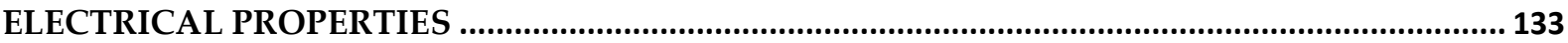

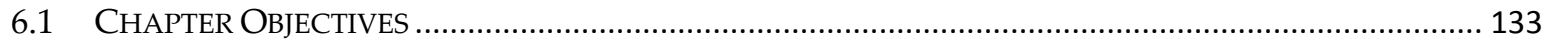

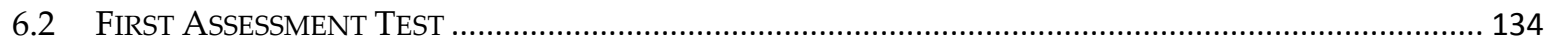

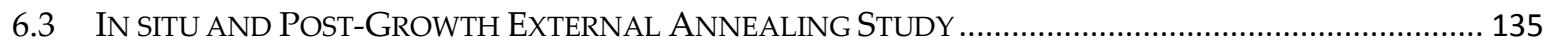

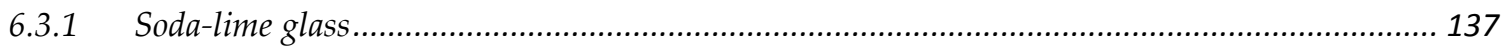

6.3.2 In situ anneal of borosilicate and sapphire samples.............................................................. 141

6.3.3 Post-growth anneal of borosilicate and sapphire samples .................................................... 147

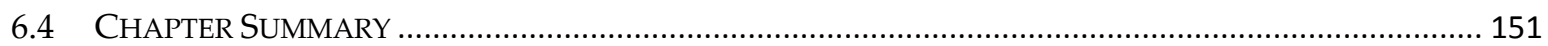

7 RESULTS C: EFFECTS OF THERMAL ANNEALING ON THE METAL CONTACTS............ 153

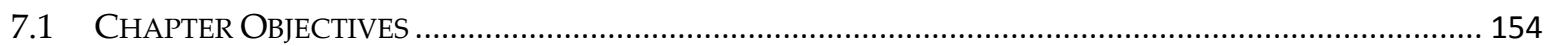

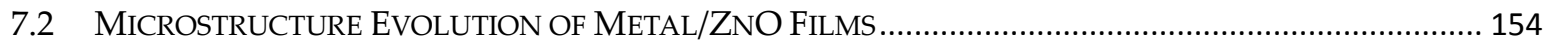

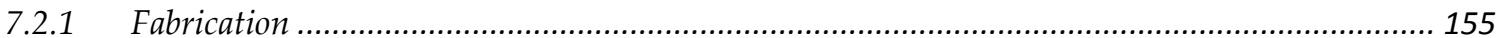

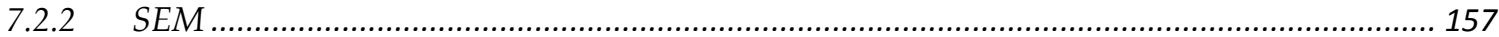

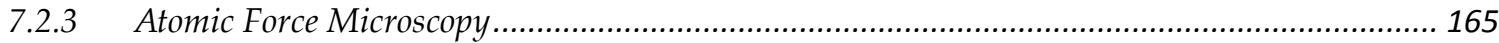

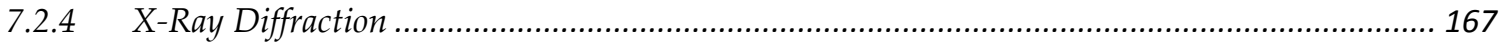

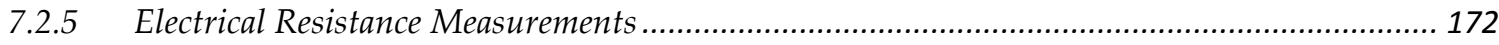

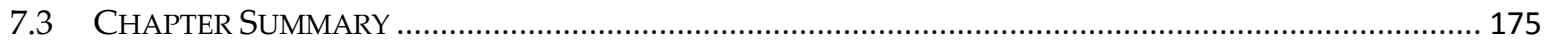

$8 \quad$ RESULTS D: CONDUCTIVE ZINC OXIDE CONTACTS ......................................................... 177

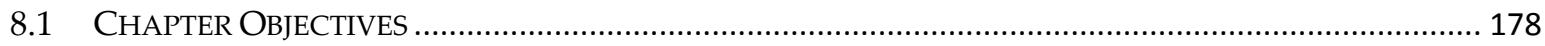




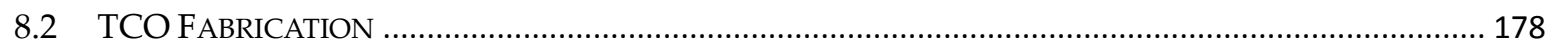

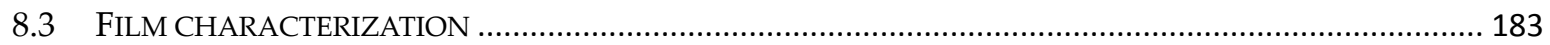

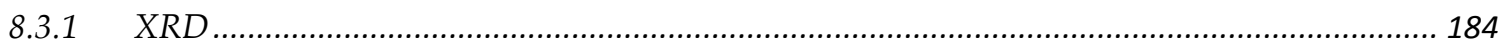

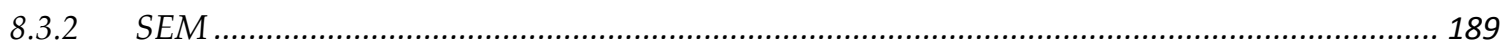

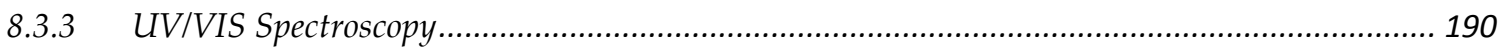

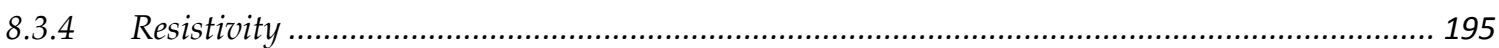

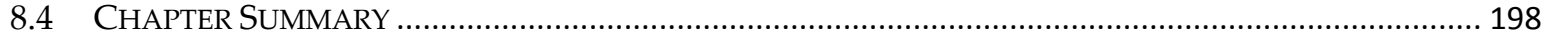

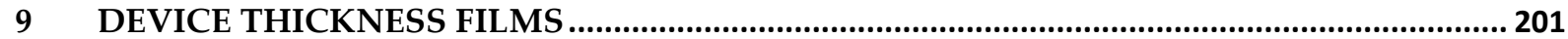

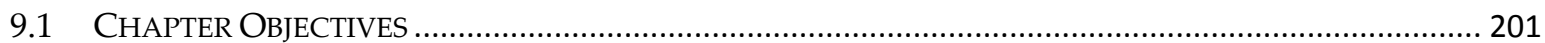

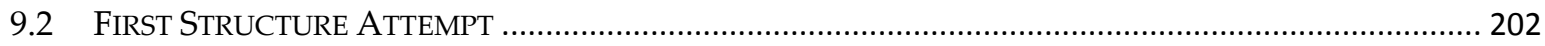

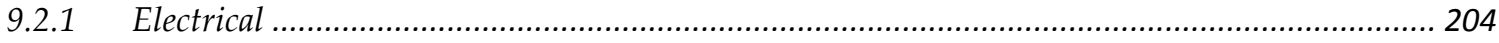

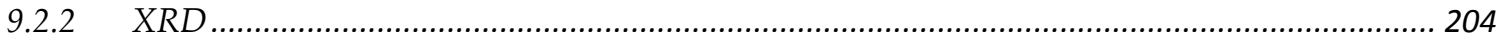

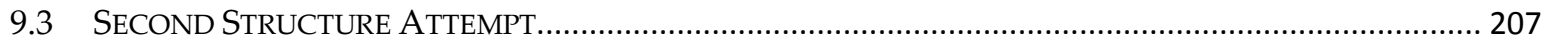

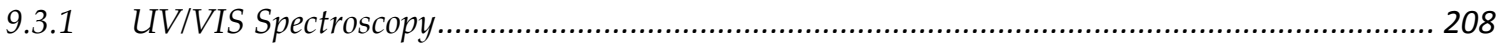

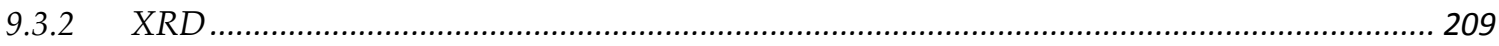

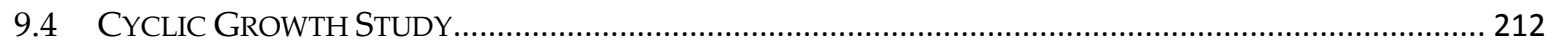

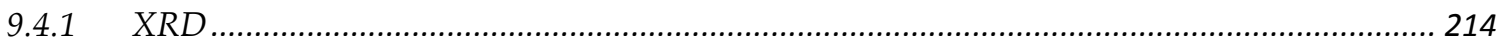

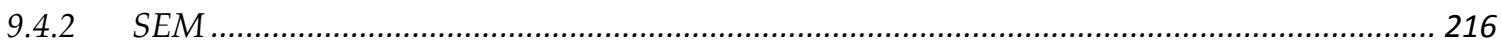

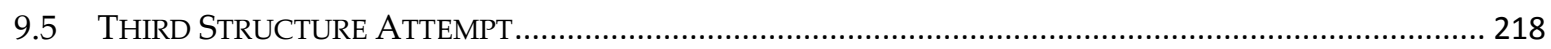

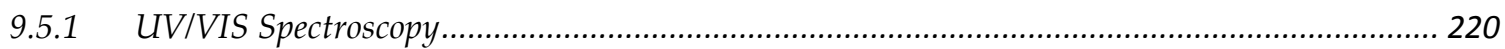

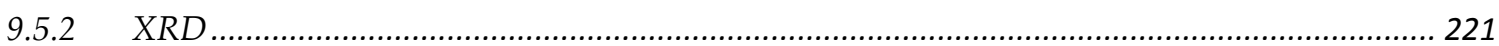

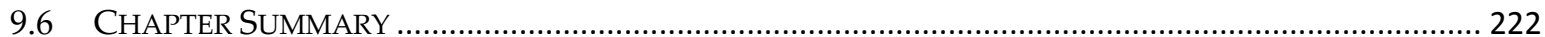

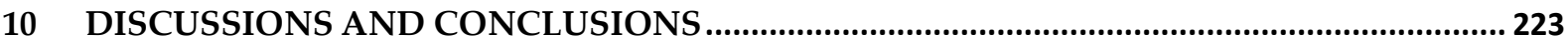

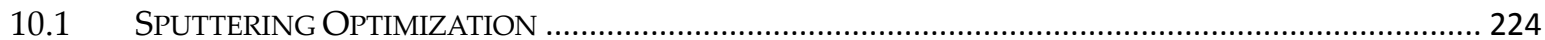

10.2 THERMAL ANNEALING EFFECTS ON THE ZNO LAYER.................................................................... 225

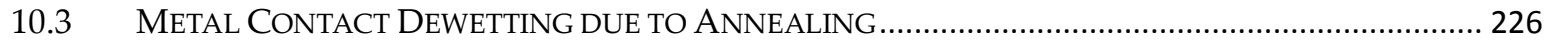

10.4 TRANSPARENT CONDUCTIVE OXIDE CONTACTS.......................................................................... 227

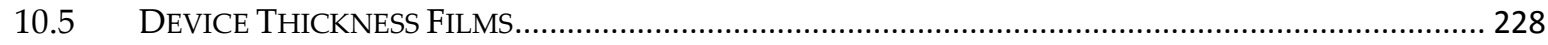

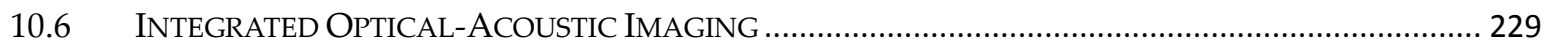

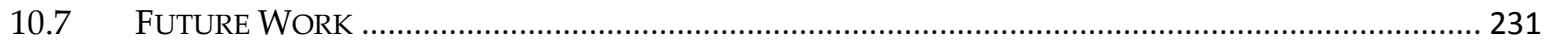

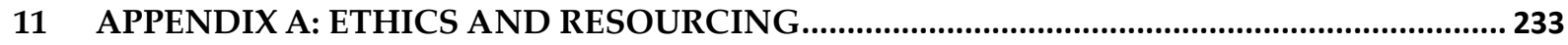




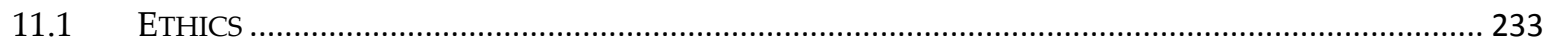

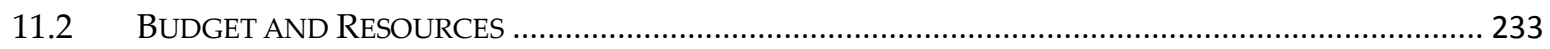

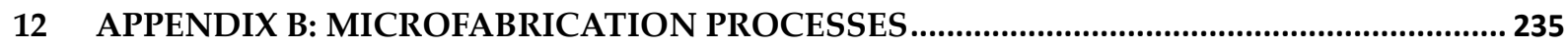

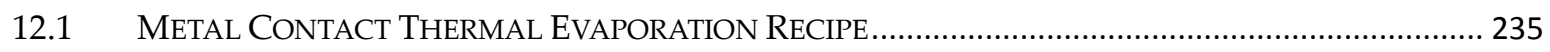

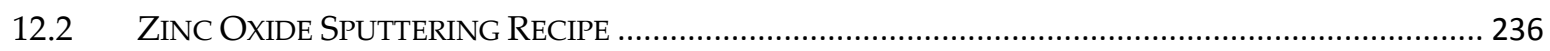

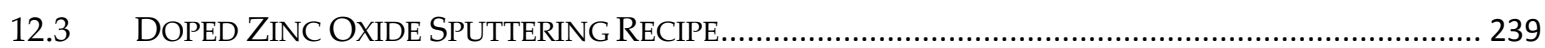




\section{Figures}

Figure 1.1: The ultrasound application spectrum ..................................................................... 2

Figure 1.2: A schematic representation of a simple piezoelectric resonator .................................. 4

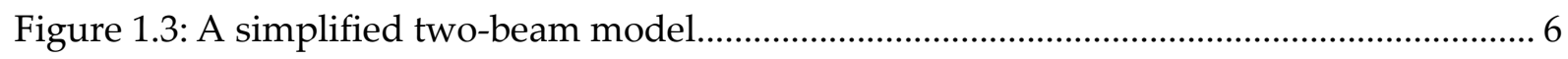

Figure 2.1: A simplified schematic showing piezoelectric action in a crystal........................... 18

Figure 2.2: A diagram illustrating the relations ........................................................................ 21

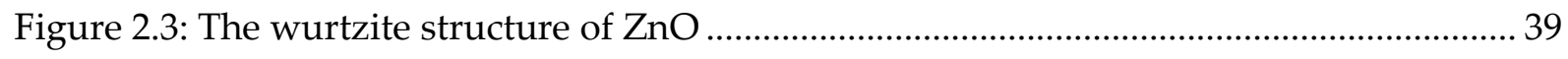

Figure 2.4: Main low indexed crystal planes in a hexagonal structure...................................... 40

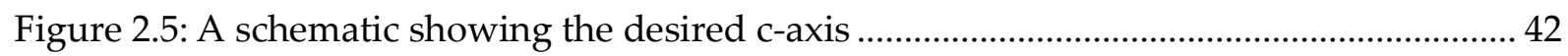

Figure 3.1: Schematic showing our simplified transducer structure ......................................... 49

Figure 3.2: A schematic drawing showing the superposition ...................................................... 56

Figure 3.3: Deposited films can possess tensile or compressive ................................................ 58

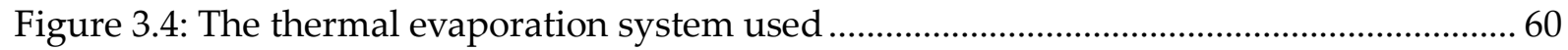

Figure 3.5: A simplified schematic of a DC sputtering system ................................................... 64

Figure 3.6: A simplified schematic of an RF magnetron sputtering system ............................. 67

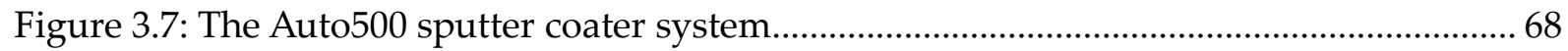

Figure 3.8: A schematic showing the external annealing setup ............................................... 70

Figure 4.1: A schematic illustrating the operation of the Dektak profiler .................................. 72

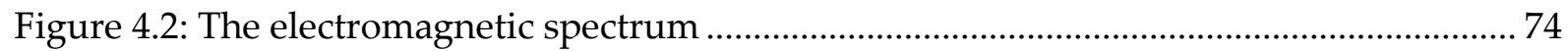

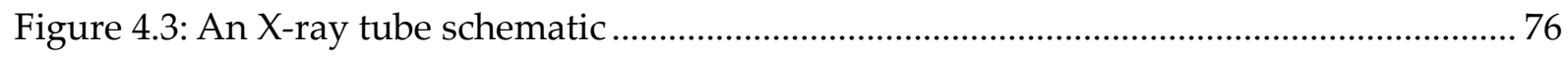

Figure 4.4: Top schematic shows the most common electronic transitions ............................... 77

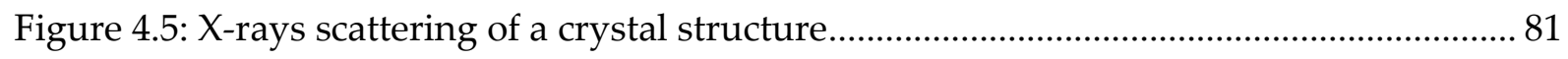

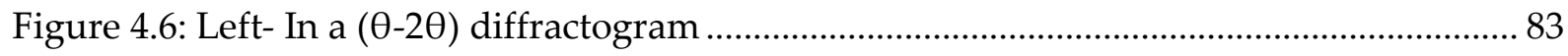

Figure 4.7: Sample displacement error gives incorrect peak positions....................................... 85 
Figure 4.8: The effects of different strain types on diffraction

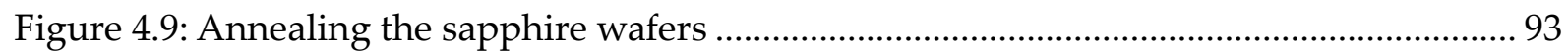

Figure 4.10: A schematic showing the setup used to measure the electrical ............................. 95

Figure 4.11: A schematic diagram showing the simplified structure of a UV/VIS ................ 101

Figure 4.12: The transmittance spectrum of a ZnO film .......................................................... 102

Figure 4.13: The Tauc plot for a zinc oxide sample .................................................................... 105

Figure 5.1: Schematic showing the different processing steps................................................ 108

Figure 5.2: Main extrinsic variables and parameters affecting ................................................ 111

Figure 5.3: Main intrinsic variables and parameters affecting .............................................. 112

Figure 5.4: A schematic showing the deposited transducer structure ..................................... 120

Figure 5.5: Properties of the deposited $\mathrm{ZnO}$ buffer layers on top …....................................... 121

Figure 5.6: Each batch had two adjacent soda-lime substrates ............................................... 123

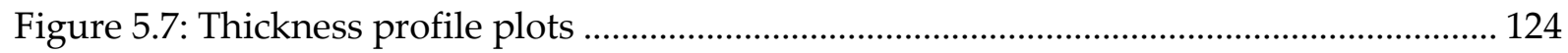

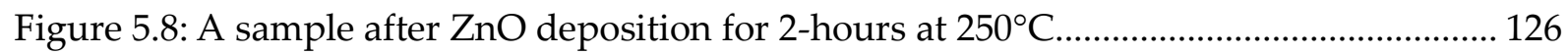

Figure 5.9: Crystal orientation selectivity of $\mathrm{ZnO}$ films ......................................................... 127

Figure 5.10: The plots show the effect of adding oxygen ....................................................... 129

Figure 6.1: Film peeling is clearly visible on top of ................................................................. 135

Figure 6.2: X-ray diffractograms of the different $\mathrm{ZnO}$ samples............................................... 138

Figure 6.3: The estimated residual stress levels for the different $\mathrm{ZnO}$.................................... 139

Figure 6.4: The average measured electrical resistivity values ................................................. 140

Figure 6.5: Plots showing the estimated structural properties.................................................. 143

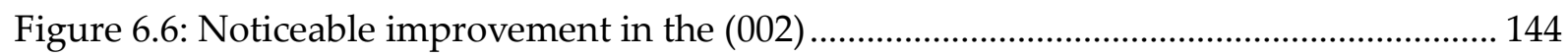

Figure 6.7: Plots showing the structural properties of the different ......................................... 145

Figure 6.8: Noticeable improvement in the (002) crystal ......................................................... 146

Figure 6.9: Stress for films deposited on sapphire versus ..................................................... 148

Figure 6.10: The estimated CDS of the ZnO (002) peaks.......................................................... 149

Figure 6.11: The estimated (002):(101) selectivity ................................................................... 150

Figure 7.1 A sketch highlighting the different fabrication steps............................................. 157

Figure 7.2: Top-view secondary electrons SEM micrographs of ............................................. 158

Figure 7.3: Top-view backscattered electron SEM micrographs............................................... 160 
Figure 7.4: Top-view backscattered electron SEM micrographs of $\mathrm{Cr} / \mathrm{Au} / \mathrm{Cr}$ films 161

Figure 7.5: Top-view backscattered electron SEM micrographs of $\mathrm{ZnO}$ films 162

Figure 7.6: Top-view SEM micrographs of $\mathrm{ZnO}$ films 164

Figure 7.7: AFM images of the exposed metal contact 165

Figure 7.8: AFM images showing the grains of the $\mathrm{ZnO}$ films 166

Figure 7.9: AFM measurements plot showing $\mathrm{ZnO}$ grain growth 167

Figure 7.10: The estimated stress of the $\mathrm{ZnO}$ films. 168

Figure 7.11: The deposited $\mathrm{ZnO}$ (002):(100) selectivity 169

Figure 7.12: The estimated $\mathrm{ZnO}(002)$ crystallite domain size ..... 170

Figure 7.13: The estimated crystallite domain size of $\mathrm{Au}(111)$. 171

Figure 7.14: Measured Au (111) peak intensity for different 172

Figure 7.15: A sketch showing the impedance measurement 173

Figure 7.16: The measured surface resistance of our samples 174

Figure 8.1: Direct and processed (for clarity) sample images 179

Figure 8.2: Schematics showing the sample placement inside the sputter coater. 180

Figure 8.3: Photographs showing the different regions of each sample 183

Figure 8.4: The average $\mathrm{ZnO}(002)$ crystallite domain size. 185

Figure 8.5: The measured $\mathrm{ZnO}$ (002) peak intensity..... 186

Figure 8.6: The estimated $\mathrm{ZnO}$ (002):(100) selectivity 187

Figure 8.7: The estimated $\mathrm{ZnO}$ (002):(101) selectivity 188

Figure 8.8: SEM images of the different regions 189

Figure 8.9: The measured optical transmission level (left) and estimated bandgap. 190

Figure 8.10: The estimated bandgap in each region 192

Figure 8.11: The estimated optical bandgap in each region 193

Figure 8.12: The estimated optical bandgap in each region 194

Figure 8.13: The measured resistivity in each region 196

Figure 8.14: The measured resistivity in each region 197

Figure 9.1: The $5 \mu \mathrm{m}$ thick $\mathrm{ZnO}$ films were sputtered 203

Figure 9.2: Resistance values across the $\mathrm{ZnO}$ film at each contact 204

Figure 9.3: The estimated residual stress levels of the $5 \mu \mathrm{m} \mathrm{ZnO}$ 205 
Figure 9.4: The estimated CDS values..... 206

Figure 9.5: The (002)/(100) selectivity for the $5 \mu \mathrm{m} \mathrm{ZnO.........................................................} 206$

Figure 9.6: (left) a sketch illustrating the top view of ................................................................... 208

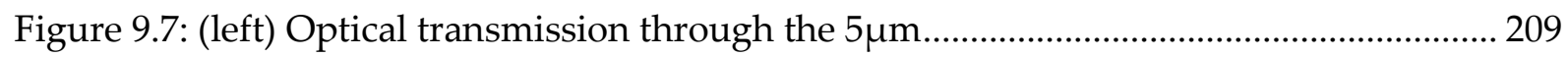

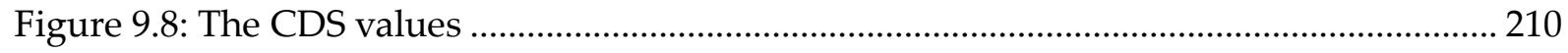

Figure 9.9: The (002)/(100) selectivity for the different layers .................................................. 211

Figure 9.10: A sketch illustrating the mask design ................................................................... 214

Figure 9.11: The average $\mathrm{ZnO}(002)$ crystallite domain size...................................................... 215

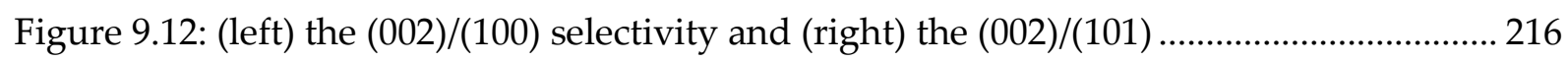

Figure 9.13: Cross-sectional and $20^{\circ}$ tilted secondary electrons SEM ...................................... 217

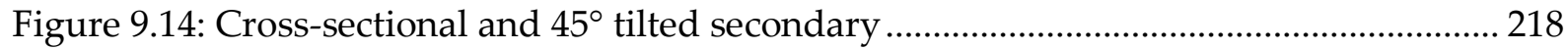

Figure 9.15: An image showing our transparent single element ............................................. 220

Figure 9.16: (left) Optical transmission through the single element ........................................ 221

Figure 10.1: Illustration of the proposed acoustic-optical lens ................................................. 230 


\section{List of symbols according to appearance}

D Active layer thickness / Parallel sample displacement / Film Thickness

$n \quad$ Integer / Diffraction order / Index of refraction

$\lambda \quad$ Resonance wavelength / Photon wavelength

$v_{L} \quad$ Propagation velocity of the longitudinal compressional wave

$f \quad$ Frequency / Emitted photon frequency

$f_{r} \quad$ Fundamental resonant frequency

$Z_{0} \quad$ Characteristic acoustic impedance

E Electric field / Energy

$\sigma \quad$ Stress

$\varepsilon \quad$ Strain

$P \quad$ Polarization density

$e \quad$ The piezoelectric polarization / Strain coefficient

$Q \quad$ Heat

$b \quad$ The thermoelastic coefficient

$T \quad$ Temperature / Transmission

a The coefficient of thermal expansion / Length of the hexagonal cell edge 
$p \quad$ The pyroelectric constant, or the electrocaloric coefficient

$S \quad$ The elastic compliance coefficient

C The elastic stiffness coefficient / Capacitance / Sellmeier Coefficient

$n$ The dielectric susceptibility constant / Principal quantum number / Number of points in an AFM scan / Index of refraction

$c \quad$ The specific heat coefficient / Speed of light / Length along the zdirection of the hexagonal cell edge

$d \quad$ The electromechanical constants

$k_{t} \quad$ The electromechanical coupling coefficient for the thickness mode

$Z_{a c} \quad$ Acoustic impedance

$p_{a c} \quad$ Acoustic pressure

$v_{p} \quad$ Phase velocity

$\rho_{d} \quad$ Density

$T_{a c} \quad$ Acoustic transmission coefficient

$R_{a c} \quad$ Acoustic reflection coefficient

$\varepsilon_{r} \quad$ Relative electrical permittivity

$D_{i} \quad$ Electric displacement field

$\varepsilon_{0} \quad$ Vacuum electrical permittivity

$\chi \quad$ Electric susceptibility

$E_{g} \quad$ Energy gap

$E_{e x} \quad$ Excitation energy 
$T_{h} \quad$ Homologous temperature

$T_{s} \quad$ Substrate temperature

$T_{m} \quad$ Melting temperature

$V_{C} \quad$ Voltage drop across the capacitance at the target contact

$V_{d} \quad$ Voltage drop across the capacitance at the substrate contact

$A_{C} \quad$ Target contact area

$A_{d} \quad$ Substrate contact area

$q \quad$ Particle charge

V Particle acceleration voltage / Voltage

$m \quad$ Mass of the particle

$h \quad$ Planck's constant / Miller's index

$v_{i} \quad$ Initial particle speed

$v_{f} \quad$ Final particle speed after deceleration

$\lambda_{\text {char }}$ Characteristic wavelength

$\Delta E \quad$ Energy gap

$\alpha \quad$ Characteristic line classification for adjacent shell transitions / Light absorption coefficient

$\beta \quad$ Characteristic line classification for transition at a shell distance of 2

$E_{m_{e}} \quad$ Electron's rest mass energy

$k \quad$ Miller's index

l Miller's index 
$d_{h k l} \quad$ Lattice distance between the parallel diffracting crystal planes having the lattice planes with the $(h k l)$ Miller indices

$\theta \quad$ The angle between the incident beam and the diffracting lattice planes

$I_{S} \quad$ Intensity of a scattered x-ray beam

$r \quad$ Distance / Transition type exponent

$I_{i} \quad$ Intensity of the incident x-ray beam

$B \quad$ Full-width at half-maximum of a diffraction peak / Sellmeier Coefficient

$R \quad$ Radius of the diffractometer's circle / Electric resistance

$\Delta 2 \theta \quad$ Peak broadening

$\varepsilon_{z} \quad \mathrm{ZnO}$ macrostrain along the c-axis

$c_{0} \quad$ The unstrained unit cell distance along the $\mathrm{z}$-direction

$t \quad$ Mean crystallite domain size

CDS Mean crystallite domain size

K Shape factor

I Electric current

$R_{q} \quad$ Root mean squared roughness

$y_{i} \quad$ Vertical deviation of the $i^{\text {th }}$ point from the mean

$R_{k u} \quad$ Measured Kurtosis

M Number of oscillations between two transmission extrema

A Sellmeier Coefficient

F Sellmeier Coefficient 
G Sellmeier Coefficient

$R \quad$ Reflection 
This page is intentionally left blank 


\section{CHAPTER 1}

\section{Introduction}

\subsection{Ultrasonics}

Sound waves are pressure oscillations that propagate through compressible media due to a sound source. The human ear can generally distinguish loud sounds in a medium, if their frequencies fall between $20 \mathrm{~Hz}$ and $20 \mathrm{kHz}$. Once the waves oscillate at higher frequencies, they will be in the superaudible or ultrasonic region and go undetected by the normal ear structure.

Ultrasonic processes involve considerations of phenomena related to the ultrasound wave propagation, and the subsequent interactions of such waves with the matter being irradiated by them [1]. The energy of ultrasound waves can affect chemical reactions, be transferred into other forms of energy, or be used in non-destructive testing. This gives rise to a broad range of ultrasonic applications in various areas of science, medicine, and industry, with the global ultrasonic technology market currently valued at 31.6 billion USD with a 
forecasted compound annual growth rate (CAGR) of 11.5\% until 2021 [2]. The main current ultrasound applications across the frequency spectrum are shown in figure 1.1. When combined with signal processing techniques, highfrequency $(>1 \mathrm{MHz})$ ultrasound transducers can be used for non-destructive testing and acoustic imaging $[3,4,5]$. Acoustic imaging usually refers to the processes that allow the creation of images of objects by means of measuring their mechanical (elastic) properties on a given spatially-correlated scale. Acoustic imaging is considered non-invasive, and provides the ability to image a sample's surface and subsurface through a live-feed $[6,7]$; hence it is a popular characterization technique for industrial and biological samples.

Wave diffraction dictates that the smallest resolvable feature in a far-field imaging technique is dependent on the applied wavelength [8]; hence, to achieve higher spatial resolution in ultrasound imaging, an increase in the ultrasound frequency is required. A shortcoming of this frequency increase is a decrease in the waves' penetration depth into the examined material, due to frequency-dependent attenuation.

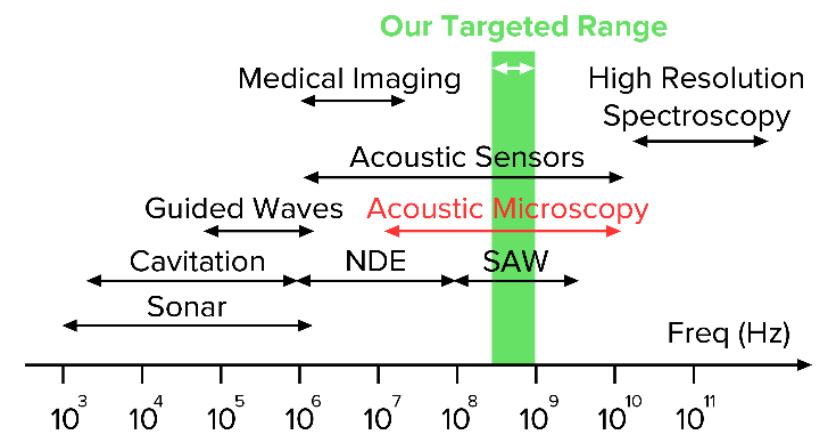

Figure 1.1: The ultrasound application spectrum, based on [9]. The targeted frequency range of operation of our thin-film structures is highlighted. 
In this project, we aim to fabricate thin-film structures, which could be used as ultrasound transducers that operate within the $300 \mathrm{MHz}-1 \mathrm{GHz}$ frequency range. These frequencies fall in the medium frequency range of acoustic microscopy [9], where a vast variety of acoustic sensors and non-destructive evaluation (NDE) techniques are carried out for both industrial and biological applications. Recent applications of acoustic microscopy include imaging blood vessel walls and the anterior segments of the eye and the skin, the characterization of neoplastic and inflammatory lesions of lymph nodes [10], and the non-invasive investigation of the mechanical properties of living cells [11].

\subsection{Ultrasound Transducers and Acoustic Microscopy Considerations}

High-frequency ultrasound waves are usually generated using the inverse piezoelectric effect, where mechanical vibrations emanate from piezoelectric materials due to an applied electric potential. The simplest piezoelectric transducer comprises a piezoelectric crystal sheet, sandwiched between two conductive contacts, as seen in figure 1.2. This structure was first proposed and fabricated by the French Professor Paul Langevin during the First World War [12]. Resonance will occur in such a simple structure once the phase of the reflected longitudinal wave matches that of the incident longitudinal wave, to allow the waves to reinforce. Thus, for a piezoelectric element operating in the thickness mode and having a thickness $D$, the resonance condition is given by:

$$
2 \times D=n \times \lambda=\frac{n \times v_{L}}{f}
$$

Equation 1.1 
where $n$ is a positive integer, $\lambda$ is the resonance wavelength, $v_{L}$ is the propagation velocity of the longitudinal compressional wave through the piezoelectric material, and $f$ is the resonant wave frequency.

Therefore, the smallest thickness $D_{\min }$ that satisfies resonance at the fundamental frequency $f_{r}$ is given by substituting $n=1$ in the previous equation:

$$
D_{\min }=\frac{v_{L}}{2 \times f_{r}}
$$

Equation 1.2

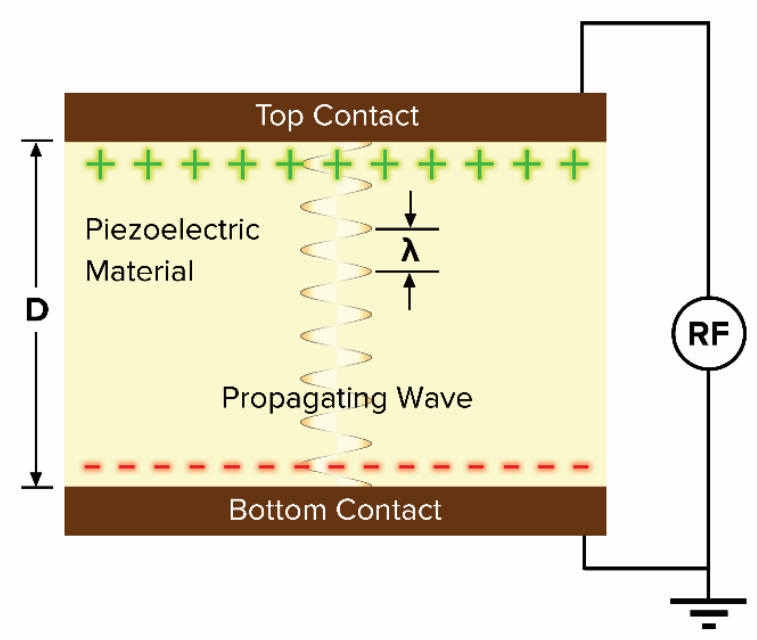

Figure 1.2: A schematic representation of a simple piezoelectric resonator

High-frequency ultrasound transducers used for acoustic microscopy typically resonate at frequencies higher than $50 \mathrm{MHz}[11,13,14]$, which means that the resonant structure will only be several tens of micrometres long, depending on the piezoelectric material of choice (table 1.1). 
Table 1.1: Acoustic impedance $\left(Z_{0}\right)$ and longitudinal wave velocity $\left(v_{L}\right)$ values for some popular piezoelectric materials. The required transducer thickness is calculated for each material based on equation 1.2 for three frequencies, $15 \mathrm{MHz}, 300 \mathrm{MHz}$, and $1 \mathrm{GHz}$. Data is taken from $[9,15,16,17]$

\begin{tabular}{llllll}
\hline \hline Material & $\boldsymbol{Z}_{\mathbf{0}}(\mathrm{MRayl})$ & $\boldsymbol{v}_{\boldsymbol{L}}\left(\mathbf{1 0 ^ { 3 }} \mathbf{\mathbf { m }} / \mathbf{s}\right)$ & $\boldsymbol{D}_{\mathbf{1 5 M H Z}}(\boldsymbol{\mu m})$ & $\boldsymbol{D}_{\mathbf{3 0 0 M H z}}(\mu \mathrm{m})$ & $\boldsymbol{D}_{\mathbf{1 G H z}}(\mu \mathrm{m})$ \\
\hline Quartz (X-cut) & 15.21 & 5.74 & 191.3 & 9.56 & 2.87 \\
Rochelle salt & 5.48 & 3.1 & 103.3 & 5.16 & 1.55 \\
$\mathrm{ADP}$ & 5.85 & 3.25 & 108.3 & 5.41 & 1.62 \\
$\mathrm{LiNbO}$ ( $\left(36^{\circ}\right.$ Y-cut) & 34.15 & 7.36 & 245.1 & 12.26 & 3.68 \\
$\mathrm{AlN}$ & 32.68 & 10.12 & 337.3 & 16.86 & 5.06 \\
$\mathrm{PVDF}$ & 4.12 & 2.3 & 76.7 & 3.83 & 1.15 \\
$\mathrm{ZnO}$ & 35.95 & 6.33 & 211 & 10.55 & 3.16 \\
$\mathrm{PZT}(5 \mathrm{H})$ & 35.65 & 4.6 & 15.3 & 7.66 & 2.3
\end{tabular}

The first scanning acoustic microscope (SAM) was developed by Lemons and Quate in 1973 [18]. This microscope was driven by $35^{\circ}$ Y-cut $\mathrm{LiNbO}_{3}$ transducers that operated in their fifth harmonic at a frequency of $160 \mathrm{MHz}$ [18], and was essentially a broadband scanned ultrasonic imaging system that used a spherical lens of high F-number to image shallow details in opaque samples, with a diffraction limited lens resolution of $10 \mu \mathrm{m}$ [9].

In a Lemons-Quate type SAM, electrical pulses of a single radio frequency $(\mathrm{RF})$ are applied to the piezoelectric transducer on top of the acoustic lens, as shown in figure 1.3. The transducer would then convert the electrical RF pulse into an ultrasonic wave having the same frequency, which is coupled to the lens structure. These acoustic waves are focused by means of a polished spherical cavity at the opposite face of the lens. Thereafter, a liquid, usually water, is used to acoustically couple the lens cavity to the sample surface. Based 
on the acoustic properties of the sample, a portion of the acoustic signal is reflected through the same path.

Once at the ultrasound transducer, the inverse piezoelectric effect converts the reflected ultrasound wave into an electrical pulse, which is electronically processed and fed into a computer imaging system. To form an image, the lens is mechanically moved from one point to another in a raster fashion, with the measurement and acquisition processes being repeated for each point.

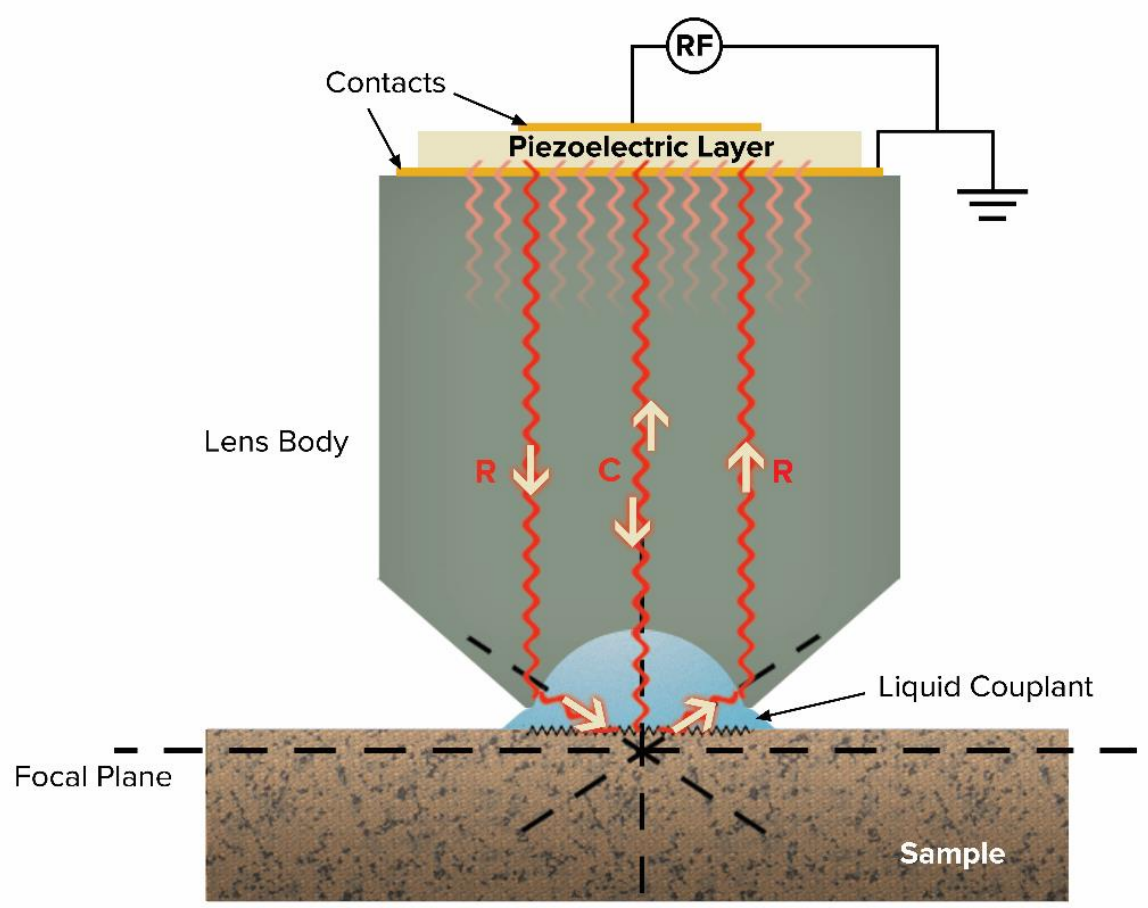

Figure 1.3: A simplified two-beam model showing the structure and the longitudinal wave-propagation in a scanning acoustic microscope. $\mathrm{C}$ represents the central beam that is reflected onto the same path, whereas $\mathrm{R}$ represents the outside cone of rays. 
As acoustic microscopy is usually performed at a single frequency and on a fixed axis, most of the usual aberrations that complicate the design of optical microscopes are absent. Spherical aberrations are minimized by making sure that the wave propagation velocity through the couplant liquid is slower than the acoustic velocity of the lens, where velocity is proportional to the wavelength for a given frequency. Due to such inherent advantages, SAM devices often offer imaging at spatial resolution values close to that of the ideal theoretical limit.

When the ultrasound wavelength is decreased, the spatial resolution is increased; however, acoustic losses due to scattering and mismatches are increased even faster [9]. Thus, a main design consideration of acoustic microscopes is the reduction of system losses.

Transducers suited to acoustic microscopy are required to generate a high mechanical power density, provide a sensitive detection for the reflected signal, and operate within the targeted frequency range [9]. Current SAM systems rely on utilizing the higher frequency harmonics for ultrasound generation and detection, which imposes severe limitations to the contrast and signal level [18]. This deficiency is directly related to the status quo of transducers fundamentally operating at these frequencies, with very few options being commercially available when the operation frequency exceeds $200 \mathrm{MHz}$, as the fabrication of even simple single-element transducers poses a lot of difficulties and challenges to overcome [11]. Moreover, the integration of such transducers in final device structures places stern design and processing considerations to achieve the required specifications. Hence, there still is a considerable room for improvement in terms of high-frequency piezoelectric transducer fabrication and device integration $[19,20]$, while there is no reason 
to believe that such transducers will not be commercially available in the near future [11].

The fabrication of piezoelectric ultrasound transducers usually relies on tools cutting down, milling, lapping, and bonding a thick bulk of a piezoelectric crystal, until the desired shape and dimensions are achieved; i.e., top-down microfabrication. The dimensional constraint of high-frequency ultrasonic transducers, as shown in table 1.1, implies that a piezoelectric element has to be delicately thin, which places stringent limits that exceed the tolerances of most top-down fabrication techniques [21, 22].

On the other hand, bottom-up microfabrication, with its high level of dimensional control, provides an array of promising techniques and tools to facilitate the making of high-frequency ultrasound transducers in the $\mathrm{GHz}$ range. However, achieving a good level of control over bottom-up processes is difficult when the area and thickness of the fabricated films increase, since film uniformity, stress and cracking, as well as crystal defects become problematic $[23,24]$. For example, this limit is around one micrometer for simple structured lead-zirconate-titanate (PZT) piezoelectric films [25, 23, 26].

As shown in table 1.1, our targeted transducers frequency range of $300 \mathrm{MHz}-1 \mathrm{GHz}$ requires an active film thickness of around $1-10 \mu \mathrm{m}$ for most conventional thin-film piezoelectric materials, such as aluminium nitride (AlN), PZT, and zinc oxide ( $\mathrm{ZnO})$. Thus, neither top-down nor bottom-up microfabrication techniques are well-suited for the creation of such piezoelectric devices, where very limited and expensive commercial options exist [27, 28, 29]. The supply scarcity of such high-frequency ultrasound transducers is not solely attributed to the limited number of experienced and well equipped producers of high-frequency ultrasound transducers, but also due to the challenging and complex considerations faced when trying to integrate the constituting films 
into final device structures [30]. Ultimately, the availability of such transducers facilitates the development of novel ultrasound applications, particularly in the sensing and acoustic microscopy fields.

\subsection{Scope of this Work}

The work of this thesis aims to develop a process to produce $\mathrm{ZnO}$ films with a thickness ranging from $3-10 \mu \mathrm{m}$, which lies in the aforementioned thickness range that is problematic to produce by either bottom-up or top-down approaches. If such films are used as piezoelectric transducers, they are expected to produce a fundamental frequency ranging from $300 \mathrm{MHz}$ to $1 \mathrm{GHz}$, an ideal range for acoustic microscopy.

Zinc oxide was selected as the material of choice for this work due to its simple crystal structure, good piezoelectric response, high electrical resistivity, and vast amounts of processing related literature [31, 32]. In addition, it does not contain toxic or harmful elements, which makes it an appealing alternative to popular PZT based piezoelectric materials.

However, if $\mathrm{ZnO}$ films are to be used as piezoelectric transducers, their structural, electrical, and chemical properties should be optimized for maximizing their active response. This requires having high degrees of uniformity and homogeneity for the films while minimizing their inherent stresses, and ensuring a good physical coupling to the contacts. Most importantly, the ZnO films should be deposited with their crystallographic caxis perpendicular to the substrate, and possess a large electrical resistance, to ensure proper piezoelectric activity. In practice, it is essential that our zinc oxide films are reproducibly created at a high deposition-rate, to enable device 
fabrication within a reasonable time-scale, and to keep the process relatively inexpensive.

Processing variables are largely determined by the selected deposition process, which also dictates the general properties of the resultant films. Sputtering is a well-established technique for depositing both conductive and dielectric layers. In this study the use of radio frequency (RF) magnetron sputtering was employed for depositing the $\mathrm{ZnO}$ films. This choice was largely motivated by the fact that a new RF sputtering system was acquired by our group approximately one year before the start of this work. An initial master's project was conducted by Kivell [22] to evaluate the deposition of thin $\mathrm{ZnO}$ films on soda lime glass substrates using this deposition system. This work showed promising results for establishing a basic $\mathrm{ZnO}$ deposition process. However, it also indicated many processing problems, particularly with regards to high levels of inherent film stress, which lead to catastrophic delamination in films thicker than $\sim 1.5 \mu \mathrm{m}$.

The current project extended the initial work by focusing on the deposition of $\mathrm{ZnO}$ films on borosilicate glass and sapphire substrates, both potential substrate candidates for ultrasound device fabrication. It aimed to optimize the deposition parameters to facilitate the creation of films with suitable structural and electrical characteristics for device fabrication. In addition, the project evaluated various anneal procedures for relieving the inherent film strain to enable the production of $\sim 10 \mu \mathrm{m}$ thick $\mathrm{ZnO}$ films. These anneal procedures led to a study of the degradation of conventional metal contacts during high temperature treatment. As a result, the potential of an all$\mathrm{ZnO}$ transducer structure was proposed. 


\subsection{Specific Contributions of this Work}

The following points are specific contributions to the general field of $\mathrm{ZnO}$ thinfilm deposition and in particular to the application of $\mathrm{ZnO}$ as a high frequency ultrasound transducer material for acoustic microscopy applications:

- A set of parameters for creating $\mathrm{ZnO}$ films at high deposition rates in a small RF magnetron sputtering system was developed. It is concluded that these deposition conditions are particularly challenging, given the lack of the substrate holder rotation and the short target-to-substrate distance, which lead to high fluxes of energetic ions within the plasma and high levels of inherent film stress. Post-deposition anneal procedures were developed to minimize the strain in these films.

- The identification and examination of metal contact degradation during high temperature annealing steps, which eventually led to the complete dewetting of the metal contacts. This catastrophic failure shows that conventional metallic ohmic contacts for $\mathrm{ZnO}$ based devices are not suitable if high temperature anneal steps are required.

- In order to solve the latter metal contact dewetting problem, an all-ZnO transducer structure was proposed and shown to be feasible. In this structure, the traditional metal contacts used in ultrasound transducers are replaced with highly doped $\mathrm{ZnO}$ thin-films. Such a structure provided improved properties of the active $\mathrm{ZnO}$ layer, as well as a solution to the metallic contact dewetting problem. Most importantly, this structure holds the potential for developing a novel imaging device, which enables simultaneous ultrasonic and optical imaging of a given specimen. The dual functionality [33, 34, 35] of such a device is highly 
useful for sample characterization, as many NDE and medical related characterization techniques already rely on the combination of both imaging modalities, using separate acoustic and optical microscopes [10].

\subsection{Thesis Outline}

\subsubsection{Chapter two}

An introduction to the history of piezoelectricity is provided, and the relations between piezoelectricity, pyroelectricity, and thermal expansion are supplied. In addition, the piezoelectric material parameters that are relevant to electromechanical transduction applications are presented. A brief historical overview of piezoelectric materials and applications is then provided, followed by a discussion that highlights the main piezoelectric material candidates suited for thin-film applications. We state the advantages and disadvantages of each candidate material, and present an argument to justify our material selection. Finally, the status quo of high-frequency ultrasound transducers is described; thereby reflecting the lack of any commercial transducers capable of operating at our targeted frequency range. This justifies the project motives, and clarifies our contribution to the field of ultrasonics.

\subsubsection{Chapter three}

The materials and tools used to fabricate our thin-film transducer structures are described in this chapter. This includes introductions to physical vapour deposition, thermal evaporation, sputtering, and thermal annealing. Thin-film growth modes and the relevant morphological structure zone models are also introduced. 


\subsubsection{Chapter four}

The tools and methods used to characterize and inspect the produced samples are listed and described in this chapter. This includes an introduction to $\mathrm{x}$-ray diffraction, atomic force microscopy, electron microscopy, electrical impedance measurement, ultraviolet-visible spectroscopy, and other used equipment.

\subsubsection{Chapter five}

This chapter highlights the microfabrication steps involved in creating our targeted transducer structures. It also provides an insight to the previous relevant work done at VUW. Finally, the chapter presents the results of several studies aimed at optimizing the different processing parameters for zinc oxide deposition. These parameters included the structure of the bottom contact, the purity of the sputtering target, the substrate-target placement, the substrate temperature during sputtering, and the composition of the sputtering gas mixture.

\subsubsection{Chapter six}

In this chapter, the results concerning the effects of thermal annealing on the deposited $\mathrm{ZnO}$ film's structure are presented. Both in situ and post-growth external annealing steps were examined for samples having sapphire and borosilicate glass substrates. During in situ annealing, both argon and oxygen gases were used, with a varying composition of the gas mixture, while a fixed argon to oxygen ratio was used during external annealing. 


\subsubsection{Chapter seven}

This chapter investigates the limitations due to having metallic contacts for our proposed transducer structure. Thus, the effects of thermal annealing on the bottom contact layer are examined. In addition, the study compares the thermal evolution of bare metal films to metal films capped with a $\mathrm{ZnO}$ layer.

\subsubsection{Chapter eight}

In this chapter, several processing studies that aim to create transparent and conductive device contacts are presented. The candidate materials of choice to create those contacts were gallium and aluminium doped zinc oxide. X-ray diffraction, optical spectroscopy, and resistivity measurements are used to judge the quality of the films, and their suitability for our fully transparent transducer structure.

\subsubsection{Chapter nine}

This chapter provides an account for the different attempts we carried out to

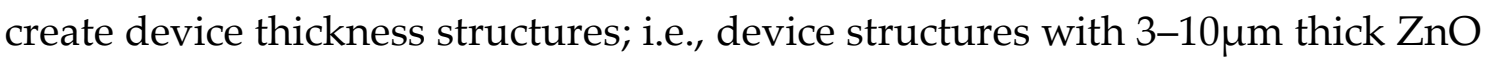
films. An additional study aiming to create (002) oriented thick $\mathrm{ZnO}$ films is also presented.

\subsubsection{Chapter ten}

This concluding chapter provides a summary of the different obtained results, and highlights the importance of integrated optical-acoustic imaging. In addition, various alternatives for possible future work are also proposed. 



\section{CHAPTER 2}

\section{ZnO as the Piezoelectric Material}

\subsection{Piezoelectric Theory}

In 1880, Pierre and Jacque Curie found that by varying the pressure along the hemihedral axes of certain crystals such as zinc blende, tourmaline, Rochelle salt, and quartz, electric polarization was produced, where measureable electric charges accumulated on certain portions of the crystals' surfaces [36]. The polarization was found proportional to the applied strain, and changed sign with it. Great interest was immediately aroused in the scientific community, and Hankel proposed piezoelectricity as a name for the effect; the name was promptly accepted by the Curie brothers [37]. This phenomenon became known as the direct piezoelectric effect. Conversely, in 1881 Lippmann predicted the inverse piezoelectric effect [37], which occurs when an electric charge is applied to a piezoelectric crystal, yielding a mechanical response. This was verified later 
that year by the Curie brothers, who showed that the piezoelectric coefficient had the same value for both the direct and inverse (indirect) effects.

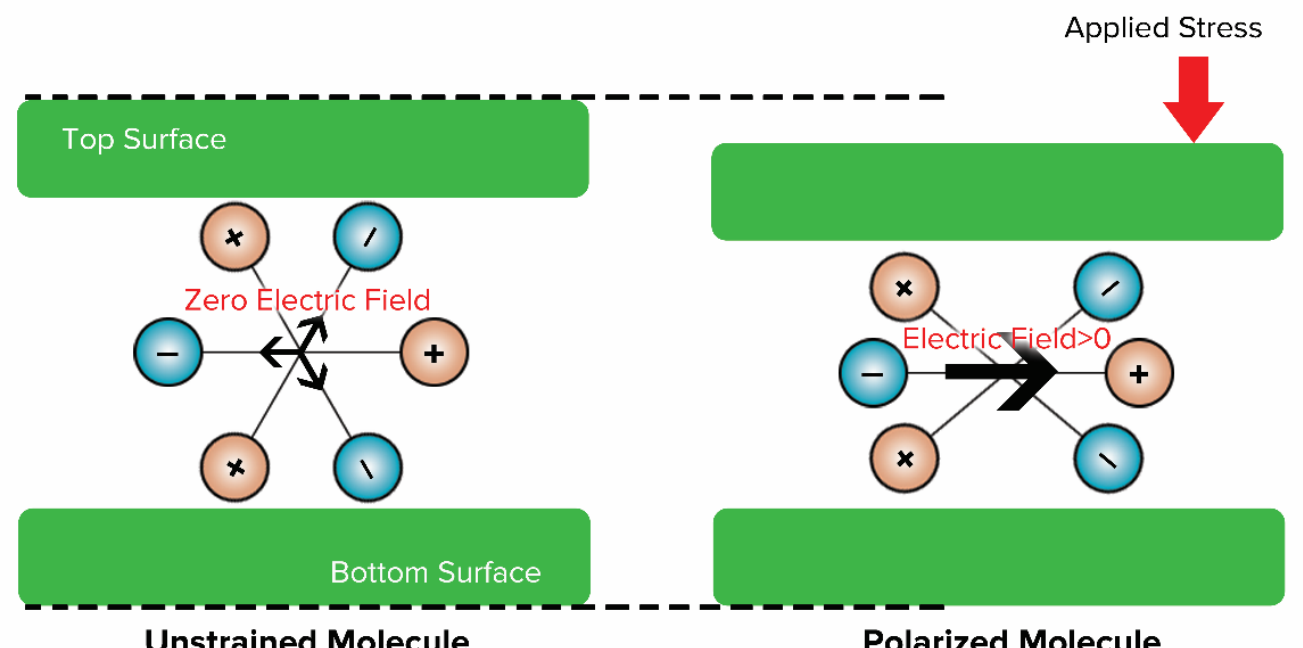

Unstrained Molecule

Polarized Molecule

Figure 2.1: A simplified schematic showing piezoelectric action in a crystal. By applying stress in a given direction to a neutral non-centrosymmetric crystal, the crystal deforms, and the centres of the cations and anions separate. This generates electric dipole moments, and a non-zero net electric polarization results.

The piezoelectric formulation was developed rigorously by Woldemar Voigt in 1894, where he combined the elements of symmetry of elastic tensors and of electric vectors with the geometrical symmetry elements of crystals [37]. He also showed which of the 32 crystal classes were piezoelectric, and for each class determined the non-zero piezoelectric coefficients [38].

Using x-rays, Bragg and Gibbs arrived at a qualitative explanation of the piezoelectric polarization in a quartz crystal in 1925 [39]; hence x-ray analysis was proven to be an essential tool for developing an atomic theory of 
piezoelectricity. In the 1940s [40], it was found that the piezoelectric effect could exist in polycrystalline ferroelectric ceramics, through the poling process. During poling, the ceramic is heated just above the Curie temperature, and then allowed to cool down slowly in the presence of a strong electric field (poling field), applied in a direction in which the piezoelectric field is required. As a result of such process, an initially macroscopically centrosymmetric ceramic loses the inversion centre and becomes piezoelectric.

It should be noted that for single crystals, the crystallographic axes $x, y$, and $\mathrm{z}$ are sometimes represented by the numbers 1-3 respectively. A crystal plate cut with its surface perpendicular to the $\mathrm{x}$-axis of the crystal lattice is called x-cut, and so forth. In the case of a piezoelectric ceramic, the z-axis is defined as the direction in which the ceramic is polarized [41].

Langevin's piezoelectric transducers relied on the indirect effect to produce powerful ultrasonic waves for sonar systems development [12]. While this effect remains to be the most dominant phenomena used for ultrasound generation, other mechanisms are also used to produce acoustic waves in materials, notably the magnetostrictive effect in ferroelectric materials, which was identified by James Joule in 1842 [42]. However, magnetostrictive and electromagnetic acoustic transducers generally suffer from high insertion losses when compared with piezoelectric transducers [28]. This limits their usage to applications that require couplant-free operation such as high-temperature ultrasonics, or the ability to generate elastic modes that are otherwise difficult [28]. Moreover, the practical upper frequency limit for electromagnetic acoustic transducers is in the region of 5 to $20 \mathrm{MHz}$ [28], which limits their usage to medium and low frequency ultrasonics. 
Currently, piezoelectricity is mainly applied in ultrasonics applications $[43,44,45,46,3]$, as well as piezoelectric sensors [47] and energy harvesting devices $[48,49]$.

\subsection{Piezoelectric Relations}

To have a better understanding of the piezoelectric effect in a given structure, it is essential to consider the interactions of this effect with other natural phenomena; namely the pyroelectric and the thermal expansion effects. The simplified relationships between these three effects are illustrated in figure 2.2, and are given by [37]:

- An electric field $E$ will cause piezoelectric stress $\sigma$. This stress is given by $\sigma=-e E$, where $e$ is the appropriate piezoelectric stress tensor.

- A strain $\varepsilon$ in a given direction will cause a non-zero net electric polarization density $P$ to exist, given by $P=e \varepsilon$, where $e$ is the appropriate piezoelectric polarization coefficient.

- A stress $\sigma$ will cause a variation in the volume of a structure, which will affect its thermal properties (variation in the quantity of heat $Q$ for a constant steady-state temperature). This thermoelastic effect is described by $\delta Q=b \sigma$, where $\delta Q$ is the heat quantity variation, and $b$ is the appropriate thermoelastic coefficient.

- A variation in the temperature $\delta T$ will cause thermal expansion or contraction to take place, thereby yielding a net strain $\varepsilon$. This is given by $\varepsilon=a \delta T$, where $a$ is the coefficient of thermal expansion. 
- The pyroelectric constant $p$ relates the change in temperature $\delta T$ with the net polarization $P$, where $P=p \delta T$.

- When an electric field $E$ is applied to a structure, a change in the total heat $\delta Q$ is observed due to the electrocaloric effect. This is given by $\delta Q=$ $p E$, where $p$ is the appropriate electrocaloric coefficient. It should be noted that this phenomenon is usually described through a relation between the change of the temperature $T$ and $E$.

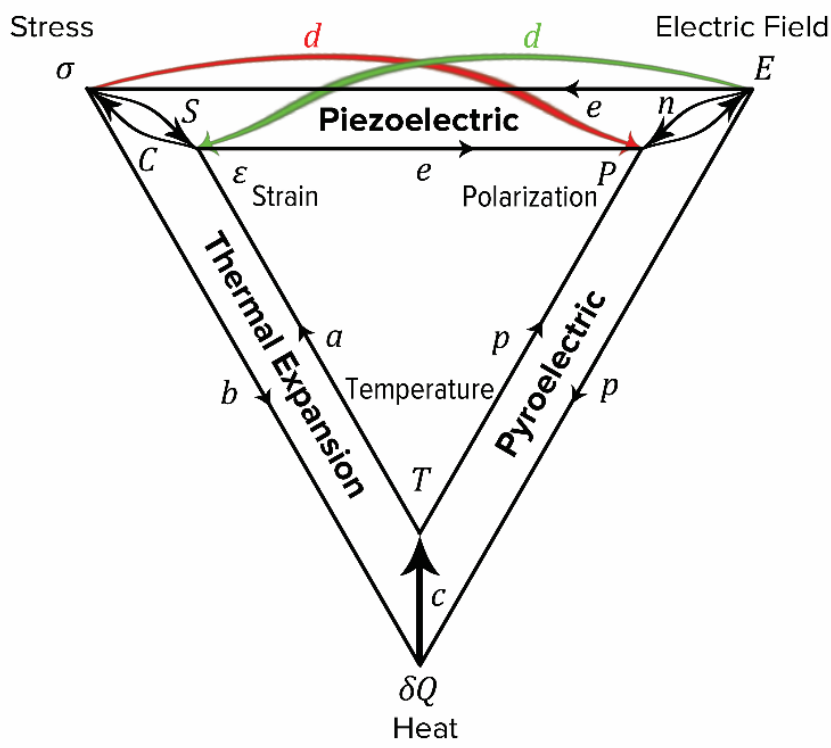

Figure 2.2: A diagram illustrating the relations between the elastic, dielectric, and thermal phenomena, adopted from Heckmann [50].

- When a stress $\sigma$ is applied, a resulting strain $\varepsilon$ is observed. This is related through the appropriate elastic compliance coefficient $S$, where $\varepsilon=S \sigma$. 
- Similarly, when a strain $\varepsilon$ exists within a structure, a stress $\sigma$ results. This is described by Hooke's law $\sigma=C \varepsilon$, where $C$ is the appropriate elastic stiffness coefficient.

- The dielectric susceptibility constant $n$ relates an applied electric field $E$ with the resulting polarization $P$. Similarly, the specific heat coefficient $c$ relates the variation in the heat stored within a structure $\delta Q$, to the resulting temperature change $\delta T$.

The effects described above are all considered to be primary effects. In every case however, there exists at least one alternative path through which the process can take place; those roundabout effects are called secondary effects. For a more thorough discussion of the relations governing these effects, please refer to Cady's book [37].

For a more thorough and realistic modelling, the primary effects of piezoelectricity should consider three different types of quantities:

- Electric field and polarization vectors, which are first order tensors.

- The second order tensors of the elastic stress and strain.

- The corresponding piezoelectric coefficients, which are third order tensors.

Hence, the generalized Hooke's law is given by:

$$
\sigma_{i j}=C_{i j k l} \varepsilon_{k l}
$$

Equation 2.1 
where $C_{i j k l}$ is a fourth rank tensor containing the elastic stiffness coefficients, $\sigma_{i j}$ is the matrix describing the stress, and $\varepsilon_{k l}$ is the matrix describing the corresponding strain. The inverse relation is written in terms of the elastic compliance coefficients $S_{i j k l}$ as:

$$
\varepsilon_{k l}=S_{i j k l} \sigma_{i j}
$$

Equation 2.2

For a piezoelectric crystal, the resulting electrical polarization $P_{i}$ due to an applied stress $\sigma_{j k}$ or strain $\varepsilon_{j k}$; i.e., the direct piezoelectric effect is given by:

$$
P_{i}=e_{i j k} \varepsilon_{j k}=d_{i j k} \sigma_{j k}
$$

Equation 2.3

where $e_{i j k}$ is the tensor describing the piezoelectric strain coefficients, and $d_{i j k}$ is the tensor describing the electromechanical constants.

Conversely, it is possible to produce a strain $\varepsilon_{j k}$ when the crystal is subjected to an electric field $E_{i}$; i.e., the inverse piezoelectric effect:

$$
\varepsilon_{j k}=d_{i j k} E_{i}
$$

Equation 2.4

By using Einstein's summation rule, $d_{i j k}$ can be reduced to $d_{k m}$, where the first subscript indicates the direction of the field, and the second subscript indicates the direction of stress. For instance, the piezoelectric strain constant $d_{33}$ relates the strain produced along the z-direction to an electric field applied along the 
same z-direction, whereas $d_{31}$ relates the strain produced in the $\mathrm{x}$-direction to an electric field applied in the z-direction, and so forth.

\subsection{Important Piezoelectric Parameters}

The advantages of using the piezoelectric effect for electromechanical transducer applications are better understood if the piezoelectric material characteristics that are most critical for performance optimization are defined.

\subsubsection{Electromechanical coupling}

The coupling constant of a piezoelectric material equals the square root of the fraction of energy converted from the electrical domain to the mechanical domain, or vice versa, in a single transduction cycle.

It is important to note that an electromechanical coupling factor will vary with the boundary conditions of a given transducer; hence, it is dependent on the vibrational modes considered, as well as the shape and dimensions of the transducer. For a bulky transducer resonating in the thickness mode, the thickness coupling constant is given by the general notation:

$$
k_{t}=\sqrt{\frac{\text { Output Mechanical Energy }}{\text { Input Electrical Energy }}}=\sqrt{\frac{\text { Output Electrical Energy }}{\text { Input Mechanical Energy }}}
$$

Equation 2.5

Coupling is a critical parameter since it limits the maximum gain and bandwidth of operation in a transducer [28]. 


\subsubsection{Acoustic impedance}

Acoustic impedance $\left(Z_{a c}\right)$ can be defined as the ratio of the acoustic pressure $p_{a c}$ to the corresponding particle velocity (phase velocity) $v_{p}$ in a given medium:

$$
Z_{a c}=\frac{p_{a c}}{v_{p}}
$$

Equation 2.6

When considering the case of longitudinal vibrational waves, the acoustic impedance of a medium is given by [51]:

$$
Z_{0}=\rho_{d} v_{L}
$$

Equation 2.7

where $Z_{0}$ is the characteristic acoustic impedance, $\rho_{d}$ is the medium's density, and $v_{L}$ is the longitudinal velocity. Acoustic impedance has a unit of $\mathrm{kg} \cdot \mathrm{m}^{-2} \cdot \mathrm{s}^{-1}$ or Rayl. The reflection of the acoustic energy that is incident normal to an interface is solely determined by the ratio of the specific acoustic impedances of the materials on each side; the better the match, the larger the vibrational energy transmission. For a pressure wave propagating through medium-1 towards a loading medium-2, at a direction normal to the interface, the transmission coefficient $T_{a c}$ is given by $[11,51]$ :

$$
T_{a c}=1-R_{a c}=1-\frac{Z_{2}-Z_{1}}{Z_{2}+Z_{1}}
$$

Equation 2.8 
where $R_{a c}$ is the reflection coefficient which represents the fraction of the reflected wave energy to the incident wave energy; $Z_{1}$ and $Z_{2}$ are the characteristic acoustic impedances of medium-1 and medium-2, respectively.

Maximum transmission $\left(T_{a c}=1\right)$ only occurs when both media have the same acoustic impedance value; i.e., matched impedance. For our ultrasound transducer application, the piezoelectric element vibrates symmetrically off the top and bottom contact surfaces. Thus, a matching layer is to be later added to the transducer's structure to improve acoustic transmission towards the load medium, while the substrate will provide support to the piezoelectric element and dampen the acoustic reverberations.

\subsubsection{Electrical permittivity}

The relative electrical permittivity $\left(\varepsilon_{r}\right)$ of a piezoelectric material determines its clamped capacitance, given its structure and dimensions. When an electric field $\left(E_{i}\right)$ is present, the bound charges within the material separate, inducing local electric dipoles. The effect of these charges is accounted for using the electric displacement field $\left(D_{i}\right)$, which is given by:

$$
D_{i}=\varepsilon_{0} E_{i}+P_{i}=\varepsilon_{0} E_{i}+n E_{i}=\varepsilon_{0}(1+\chi) E_{i}=\varepsilon_{0} \varepsilon_{r} E_{i}
$$

Equation 2.9

where $\varepsilon_{0}$ is the vacuum permittivity and $\chi$ is the electric susceptibility of the material. Just like the acoustic impedance case, matching the transducer's electrical impedance to that of the connected transmitters and receivers is essential to maximize the operational efficiency and bandwidth. 
In addition, for a given lossy piezoelectric material, electrical permittivity and conductivity provide a measure of the energy dissipation-rate during oscillation [52]. Thus, it is important to select a material that minimizes such losses.

\subsection{Piezoelectric Materials History}

Quartz and tourmaline crystals were the main piezoelectric materials of choice before World War II; however, mining naturally existing crystals was costly and location dependent. Rochelle salt was an early synthetic and cheap alternative that exhibited good piezoelectric properties [53], but its deliquescent properties meant that any transducers based on this material progressively deteriorated if exposed to damp conditions.

With the soaring demand to fabricate ultrasound transducers during World War II, intensive research was carried out to synthesize new piezoelectric materials, as high-quality quartz was hard to find or fabricate. This led to the development of new materials, notably ammonium and potassium dihydrogen phosphate (ADP and KDP), ethylene diamine tartrate (EDT), and dipotassium tartrate (DKT) [54,55]. ADP in particular, soon replaced quartz and Rochelle salt, and became widely used in sonar systems.

This ADP popularity did not last long, as polarization effects were noted in ferroelectric ceramic materials by $1945[55,56]$. Independent investigations by Von Hippel and associates at MIT [40], and by Vul and Goldman of the USSR [57] on barium titanate $\left(\mathrm{BaTiO}_{3}\right)$ confirmed that processed titania ceramics formed a new class of piezoelectric materials. These polycrystalline materials do not normally display a macroscopic piezoelectric effect as the crystallites' axes 
are randomly distributed and macroscopically averaged. However, dielectric constant measurements by the MIT group showed that the application of a high electric field across the ceramic (poling) will permanently polarize some of the randomly oriented ferroelectric domains, effectively turning the ceramic into a single ferroelectric crystal.

Then, if an alternating electric field was applied along the direction of polarization, dimensional expansion in the same direction and radial contraction in the transverse direction were observed, confirming the acquisition of piezoelectric properties. The piezoelectric response of titania based ceramics was by far superior to that of any previous piezoelectric crystal.

Titanates were relatively inexpensive, rugged, and had high piezoelectric performance. This made such ceramics very popular, where lead zirconate titanate (PZT) remains a favoured piezoelectric transducer material [25]. Later on, and for applications demanding superior piezoelectric transduction, more expensive and very high-performance novel crystals such as lithium niobate became popular [58].

A third wave of material development occurred due to the demand on piezoelectric films that can operate at higher frequencies. Zinc oxide $(\mathrm{ZnO})$ and aluminium nitride (AlN) to some extent became the standard piezoelectric films for such high-frequency applications. Both materials are complementary metal oxide semiconductor process compatible and possess low dielectric constants and low material losses, but have inferior piezoelectric properties than ferroelectric ceramics [59].

Polyvinylidine (PVDF) and other copolymers were later introduced, where such materials offered flexibility, wide bandwidth, and acoustic impedance that is highly matched to water. This made them highly favourable 
for medical ultrasonics. However, one shortcoming of PVDF was the high attenuation, which makes it unsuitable for SAW or high-frequency applications such as acoustic microscopy.

Recent improvements of piezoelectric materials are mainly due to the synergy provided by new microfabrication and material characterization techniques, and improved electronics. For example, the original PZT family has undergone numerous developments, where many application-specific PZTvariants are currently provided by suppliers [9].

\subsection{Piezoelectric Materials for Thin-films}

In this project, the piezoelectric films must possess the following properties:

- Being able to be reliably processed to films having a thickness within the range of $3-10 \mu \mathrm{m}$, and with little thickness variation and high homogeneity, to allow operation at the targeted frequency range.

- Have a high electromechanical coupling $k_{t}$.

- Have a high $d_{33}$ piezoelectric coefficient across the film thickness, to provide a strong acoustic signal in a direction perpendicular to that of the substrate surface, i.e. c-axis oriented growth in hexagonal wurtzite crystal structures (columnar) is an absolute necessity.

- Have high electrical impedance across the film contacts, to minimize the electrical current and maximize the mechanical energy path.

- Have a sufficient dielectric break-down strength to handle the maximum projected operating electric field. 
- Be strong and stable enough to withstand the processing steps such as annealing, contact deposition, plasma bombardment, etc., without flaking or peeling.

- Have a smooth and uniform surface morphology, and allow easy patterning to accommodate for the creation of relatively complex transducers in the future; i.e., annular arrays, segmented arrays, etc.

- Able to operate as a robust active layer while being driven for a large number of duty cycles before deterioration or failure; i.e., long fatigue life and a high fatigue limit.

- Allow practical device fabrication using the available equipment and resources at Victoria University of Wellington, within reasonable time and cost constraints.

In order to evaluate and compare the suitability of the main piezoelectric material candidates for our high frequency transducer application, table 2.1 provides a summary of their relevant bulk electromechanical properties.

Table 2.1: The relevant electromechanical properties of candidate piezoelectric materials [30, 9, 3, 60, 61].

\begin{tabular}{lll}
\hline \hline Piezoelectric Material & $\boldsymbol{d}_{\mathbf{3 3}}(\mathbf{p C} / \mathbf{N})$ & $\boldsymbol{k}_{\boldsymbol{t}}{ }^{2}$ \\
\hline Quartz (X-cut) & $2.3\left(d_{11}\right)$ & 0.0087 \\
LiNbO3 (36 ${ }^{\circ}$ Y-cut) & $19-27$ & 0.24 \\
AlN & 4.5 & 0.065 \\
ZnO (wurtzite) & 12 & 0.09 \\
PZT (5H) & 117 & 0.25 \\
\hline
\end{tabular}


It should be noted that the crystalline properties of thin-films vary from that of bulk material, due to the boundary conditions experienced by surface atoms. The surface energy of a solid shape is related to how well-bound the surface atoms are to the bulk atoms; typically, surface layers are less stiff and melt at lower temperatures than the solid bulk [24, 23].

Based on their electromechanical, acoustic, and electrical properties, as well as the popularity and simplicity of fabrication in thin-film form, three candidate materials were considered for this project: PZT, AlN, and ZnO. Ultimately, our piezoelectric material of choice was zinc oxide, where the following subsections provide the argument details that justify this selection.

\subsubsection{PZT}

PZT was discovered by Jaffe et al. in 1954 [62], and is a solid solution perovskite ceramic that has the chemical formula $\mathrm{Pb}\left(Z r_{x} T i_{1-x}\right) O_{3}$. PZT is a pyroelectric, ferroelectric, and piezoelectric material. It is typically produced in polycrystalline powder form, which then is press-formed into ingot shapes, sintered, poled, and finally processed into final component shapes [63].

The significance of PZT in piezoelectricity is better understood through examining its phase diagram, which is characterized by the morphotropic phase boundary (MPB) between the tetragonal and the rhombohedral phases, at a zirconate to titanate content ratio of $52 \%$ to $48 \%$. At that region, a poling field may draw upon 14 orientation states over a large temperature range, leading to exceptional ceramic polability [64]. Consequently, PZT's dielectric and piezoelectric properties show anomalous behaviours near the MPB, where the relative permittivity and the electromechanical coupling coefficients have maximal values [65]. 
Despite having high piezoelectric coefficients and dielectric strength, the commercialization and high-volume production of PZT thin-films have been delayed due to difficulties in meeting the performance and reliability requirements at an acceptable cost. However, few specialized applications such as position heads for magnetic recording, toner sensors for laser printers, fuelinjection systems, and scanning tunnelling microscopes provide the main market for PZT thin-films, as the relatively high-costs can be justified [66].

Both wet and dry processes can be reliably employed to produce PZT thin-films (table 2.2), with each process having its own advantages and disadvantages $[25,4,67]$.

Physical vapour deposition techniques offer vacuum cleanliness where high purity single crystal/epitaxial growth is possible. However, PVD suffers from slow deposition rates and difficult stoichiometry control. In contrast, chemical vapour deposition provides a high deposition rate alternative, with a well-controlled stoichiometry, but the high cost and toxicity of the involved precursors pose a real draw back for such techniques.

Table 2.2: Ferroelectric thin-film deposition techniques [25].

Dry Process

Physical vapour deposition (PVD)

Sputtering

Evaporation (e-beam, resistive, $\mathrm{MBE}$ )

Chemical vapour deposition (CVD)

Metallo-organic CVD (MOCVD)

Plasma-enhanced CVD (PECVD)

Low-pressure CVD (LPCVD)

\section{Wet Process}

Chemical solvent deposition

Sol-gel

Metallo-organic decomposition (MOD)

Chemical melt deposition

Liquid phase epitaxy (LPE) 
Wet chemical processes such as sol-gel and metallo-organic decomposition provide an excellent control over the ceramic composition at a low-cost, and are considered to be the most promising techniques for producing ferroelectric thin-films; however, film cracking is problematic during the drying process, and high temperature thermal treatment is normally required to obtain a good crystal structure.

These wet and dry deposition techniques are usually suited for forming PZT films thinner than $5 \mu \mathrm{m}$, as the preparation of thicker films poses various material processing challenges [25].

Producing thick PZT films by lapping and thinning techniques is not a viable option for thicknesses under $50 \mu \mathrm{m}$, due to the ceramic's brittleness with respect to the forces applied during processing. Moreover, the dimensional restrictions; i.e., the targeted film thickness, exceed the tolerance limits involved in top down fabrication techniques (thinning, tape-casting, screen printing, etc.) $[25,22]$.

To summarize, PVD and CVD techniques for PZT thick film fabrication have slow deposition rates, low control over the stoichiometry, require high temperature annealing, and are regarded to be too expensive [25]. In comparison, wet processes offer a lower production cost, yet the typical thickness per coating is only about 100-250nm, where high temperature treatment is required after each coating is applied to remove any organic residuals. Additional annealing/sintering steps are usually required to minimize cracking and to obtain the perovskite structure once the final film thickens $[68,69,70,71,5]$. For instance, the production of a PLZT film having a sintered thickness of $8 \mu \mathrm{m}$ requires the deposition of as many as 150 layers [72]. 
Novel techniques involving computer-controlled steps and automated processes have allowed the fabrication of thicker, crack-free films up to several microns thick [25]; however, the task is highly demanding and time-consuming, and microstructure instabilities associated with grain growth and morphology evolution in thick and dense polycrystalline films are at a higher risk [73, 74]. Aerosol deposition of PZT seems to be a promising technique to quickly create crack-free films with thickness in the range of $2-100 \mu \mathrm{m}$, nevertheless, the best obtained piezoelectric results are comparable to that of $\mathrm{ZnO}$, and further process optimization is required [75].

Thus, there is a lack of viable PZT thick film fabrication processes to provide our required transducer properties. Another reason for excluding PZT and its derivatives from consideration is the lead content, where international regulations consistently push towards the elimination of lead and other toxicants from products [76, 77].

\subsubsection{AIN}

Aluminium nitride is a high bandgap $(6.2 \mathrm{eV})$ semiconducting material [78] with a high-thermal conductivity, as high as $320 \mathrm{~W} / \mathrm{mK}$ for single crystals at $300 \mathrm{~K}$ [79]. It has a hexagonal wurtzite crystal structure, where atoms are tetrahedrally coordinated and arranged in puckered hexagonal rings. Those rings lie on the basal planes perpendicular to the crystallographic c-axis.

When stresses parallel to the c-axis are applied onto an AlN crystal, the tetrahedra deform mainly by changing the N-Al-N bond angle, rather than adjusting the Al-N bond length. This deformation causes charge separation within the crystal, which is the origin of the piezoelectric $d_{33}$ response in an AlN crystal [80]. The direction of the piezoelectric response in an AlN film 
mainly depends on the crystallite orientation, where any polarization reorientation requires breaking the primary Al-N chemical bonds; thus, aluminium nitride is not ferroelectric.

Consequently, the piezoelectric coefficients of $\mathrm{AlN}$ are stable and insensitive to variations in temperature, frequency, or amplitude of the driving electric field [80].

With regards to processing, wurtzite $\mathrm{AlN}$ is one of the rare materials that can be better synthesized in thin-film form rather than a bulk single crystal or a thick film form [81]. High quality piezoelectric AIN can be deposited by DC and $\mathrm{RF}$ sputtering [82] at very moderate temperatures, below $300^{\circ} \mathrm{C}$ [83], which is compatible for integration with most complementary metal oxide semiconductor fabrication processes [84].

These attributes make AlN an ideal material to develop thin-film piezoelectric transducers and MEMS, such as bulk and surface acoustic wave resonators $[84,80,30]$.

Despite these advantages, the electromechanical coupling $\left(k_{t}\right)$ and the piezoelectric $\left(d_{33}\right)$ coefficients of AlN are mediocre. In addition, literature suggests that the fabrication of thick piezoelectric AlN films is problematic [81, 30]. Therefore, aluminium nitride was not found to be the optimal choice for our high-frequency ultrasound transducer application.

\subsection{3 $\mathrm{ZnO}$}

The earliest production of zinc oxide $(\mathrm{ZnO})$ dates back to the Eneolithic age (5000B.C.), were it was a by-product material of copper smelting [85]. During that process the zinc proportion of a copper ore was reduced to zinc vapour, 
which then oxidized to form a non-pure form of zinc oxide. Zinc oxide became popular in the Roman Empire, as it was reduced with copper to produce brass $[86,22]$. Later on, zinc oxide powder was purified, where it was produced in wool-like form "philosopher's wool". This pure form was mainly used in ointments for medical and cosmetic purposes, as early as the first century AD [87]. By the 1800s, zinc oxide was used in paints due to its permanent whiteness, even when exposed to sun-light (Chinese white [88]), as well as providing an economical nontoxic lead alternative [86].

Interestingly, zinc was not identified until 1774 because, unlike tin and copper oxides, zinc oxide was difficult to reduce. Nowadays, zinc oxide is heated with powdered coke at $1400^{\circ} \mathrm{C}$ to produce zinc vapour, which then condenses to yield solid zinc $[88,86]$.

Soon after the first radio stations started broadcasting, $\mathrm{ZnO}$ came into popular demand due to its semiconducting properties in crystal form. Thus, by the 1920s, it was used along with an antenna and a fine copper whisker (cat's whisker) to rectify the radio's frequency signal [89]. In 1935, C.W. Bunn identified the crystal lattice parameters of $\mathrm{ZnO}$ [90], and later on, Wagner, Schottky, and co-workers in Germany proposed that the semiconducting properties of $\mathrm{ZnO}$ depended on its crystal imperfections [86]. The optical properties of $\mathrm{ZnO}$ were studied in 1954 [91], and by 1960 Hustson measured and quantified its piezoelectric properties [92]. The vibrational properties of zinc oxide were studied using Raman scattering in 1966 [93].

Despite these versatile properties, the second largest application of $\mathrm{ZnO}$ in the 1970s was photocopying, where it was added as a filler to whiten photocopying paper [94]. 
By the early 1990s, a renewed interest in $\mathrm{ZnO}$ was fuelled by the availability of high-quality substrates, and the many breakthroughs in the microfabrication and characterization techniques, such as atomic force microscopy and epitaxial growth $[23,24]$. Later, further interest was generated due to reports of ferromagnetic behaviour and p-type conduction when doped with transition metals [95, 96, 32], both of which remain controversial [97].

Concerning its properties, zinc oxide is a group II-VI piezoelectric wide bandgap semiconductor, with an energy gap $E_{g} \cong 3.4 \mathrm{eV}$ [31]. It is relatively cheap, transparent, and has a high exciton binding energy $E_{\text {ex }} \cong 60 \mathrm{meV}[98$, 97], a property that can pave the way for efficient room-temperature excitonbased lasers with low threshold currents [97]. Zinc oxide is also a polar piezoelectric material that is compatible with CMOS processes, and possesses a low dielectric constant and material losses; therefore, it allows a large bandwidth and a relatively high quality factor when used as a resonator material $[59,84]$. Moreover, several studies confirmed that $\mathrm{ZnO}$ is resistant to high-energy radiation damage $[99,100]$, and possesses an electrical resistivity $\rho$ as high as $10^{12} \Omega \mathrm{m}$ [101]. Furthermore, high quality $\mathrm{ZnO}$ crystals can be grown using simple and inexpensive techniques [31], while acids and alkalis can easily etch it [102].

This unique and favourable array of properties of zinc oxide made it a very popular material in recent device applications, particularly in optoelectronics, sensors, energy harvesting devices, piezoelectric transducers and MEMs [31, 103, 104, 105, 106]. In addition, the simple crystal structure and the stable stoichiometry of zinc oxide imply that the fabrication process of piezoelectric films is much easier when compared to more responsive piezoelectric materials such as PZT. Chemical vapour deposition, sol-gel, 
sputtering, and pulsed laser deposition can all be used to create high-quality piezoelectric ZnO films at reasonably low costs [107, 108, 109, 110].

Due to such versatile and suitable properties that match our application requirements, zinc oxide was selected to develop the active layer of our highfrequency transducer structures.

For further in-depth information on $\mathrm{ZnO}$, the readers are directed to the extensive books published by Morkoç [97] and Jagadish [89].

\subsubsection{Crystal Structure}

Under ambient conditions, zinc oxide usually crystalizes in a hexagonal wurtzite structure, as seen in figure 2.3 [111, 97]; however, zinc oxide forms a cubic zinc blende crystal when deposited onto a cubic substrate [97]. A cubic rocksalt structure of zinc oxide can result if the crystal was formed under pressures exceeding 9GPa [31].

In this project, we are only interested in the hexagonal wurtzite structure of $\mathrm{ZnO}$, since it has a superior piezoelectric response, and is thermodynamically stable [111, 112]. Wurtzite belongs to the space group $P 6_{3} m c$ in the HermannMauguin notation, and has a hexagonal unit cell with two lattice parameters $a$ and $c$, with the ratio $c / a=\sqrt{8 / 3}$, given an ideal crystal structure. 


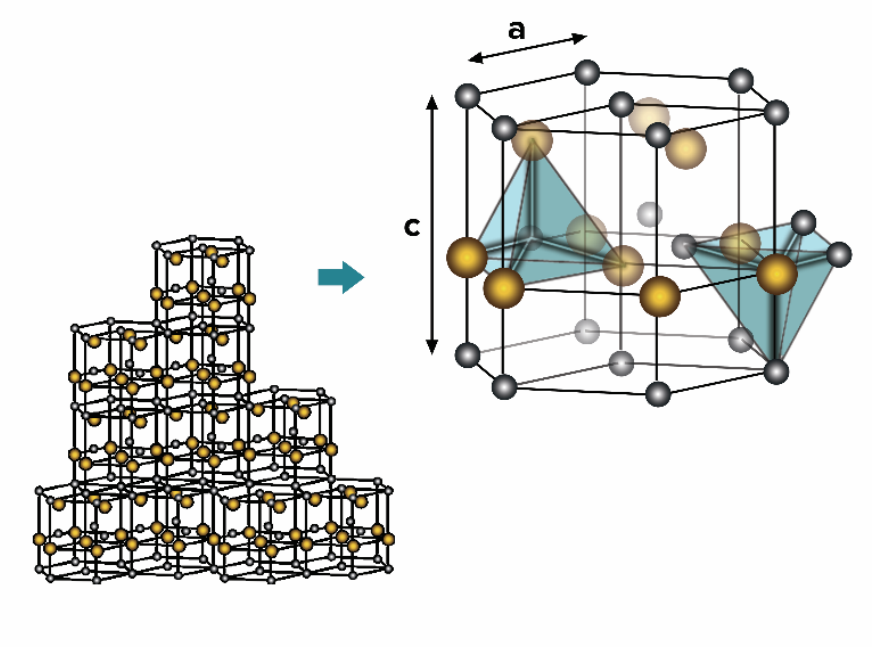

Figure 2.3: The wurtzite structure of $\mathrm{ZnO}$, with a magnified schematic at the top right section. Yellow and silver spheres represent $\mathrm{Zn}$ and $\mathrm{O}$ atoms respectively, while a and $\mathrm{c}$ are the lattice constants.

In this structure, each anion has an $s p^{3}$ covalent bond with four cations at the corners of a tetrahedron, and vice versa. However, the iconicity of zinc oxide resides at the borderline between a covalent and an ionic semiconductor [97]. Literature provides a range of values for each lattice parameter, with $3.2475 \AA \leq$ $a \leq 3.2860 \AA$ and $5.2042 \AA \leq c \leq 5.2410 \AA$ [31].

Miller-Bravais indices ( $h k i l$ ) can be used to simplify the description of the planes in a hexagonal crystal structure; this index system can be reduced to the conventional $(h k l)$ system using the symmetry $h+k=-i[22]$.

In the zinc oxide wurtzite structure, seen in figure 2.3 and figure 2.4, two interpenetrating hexagonal close-packed sublattices exist, where each lattice is composed of one element, either $\mathrm{Zn}$ or O. 


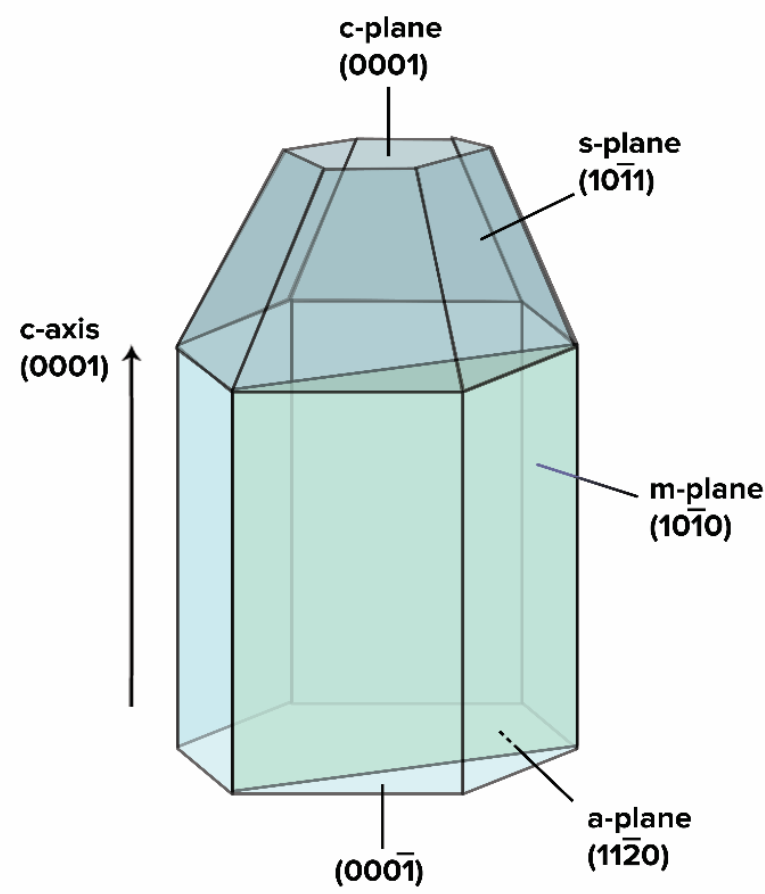

Figure 2.4: Main low indexed crystal planes in a hexagonal structure

Ideally, those lattices are displaced along the threefold c-axis by an amount $b=$ (3c/8) [97]. This structure enables polar symmetry to exist along the hexagonal c-axis, which is responsible for the piezoelectric property and the spontaneous polarization of wurtzite $\mathrm{ZnO}$ [86].

The most common surface terminations of this structure are shown in Figure 2.4. They are the Zn-polar face (0001), the O-polar face (0001), as well as the non-polar a-plane $(11 \overline{2} 0)$ and m-plane $(10 \overline{1} 0)$, which contain an equal number of zinc and oxygen atoms. These polar faces have different chemical and physical properties, as well as slight differences in the electronic structure $[113,31]$. 


\subsubsection{Mechanical and piezoelectric properties of $\mathrm{ZnO}$}

As stress is a symmetric second rank tensor, and due to its crystal symmetry, the hexagonal wurtzite phase of zinc oxide has only three independent components in its piezoelectric strain coefficients matrix; i.e., $e_{31}, e_{33}$, and $e_{15}$. Thus, equation 2.3 is given by [97]:

$$
\begin{gathered}
\left(\begin{array}{l}
P_{11} \\
P_{22} \\
P_{33}
\end{array}\right)=\left(\begin{array}{cccccc}
0 & 0 & 0 & 0 & e_{15} & 0 \\
0 & 0 & 0 & e_{15} & 0 & 0 \\
e_{31} & e_{31} & e_{33} & 0 & 0 & 0
\end{array}\right)\left(\begin{array}{l}
\varepsilon_{11} \\
\varepsilon_{22} \\
\varepsilon_{33} \\
\varepsilon_{12} \\
\varepsilon_{23} \\
\varepsilon_{31}
\end{array}\right) \\
=\left(\begin{array}{cccccc}
0 & 0 & 0 & 0 & d_{15} & 0 \\
0 & 0 & 0 & d_{15} & 0 & 0 \\
d_{31} & d_{31} & d_{33} & 0 & 0 & 0
\end{array}\right)\left(\begin{array}{l}
\sigma_{11} \\
\sigma_{22} \\
\sigma_{33} \\
\sigma_{12} \\
\sigma_{23} \\
\sigma_{31}
\end{array}\right)
\end{gathered}
$$

Hence, the induced electric polarization along the c-axis at a zero electric field, due to a uniform strain along the c-axis $\left(\varepsilon_{z}\right)$ and/or the basal plane $\left(\varepsilon_{\perp}\right)$ is given by:

$$
P_{z}=e_{33} \varepsilon_{z}+e_{31} \varepsilon_{\perp}=d_{33} \sigma_{z}+d_{31} \sigma_{\perp}
$$

Equation 2.10

The third component of the piezoelectric tensor, $e_{15}$ describes the electric polarization induced by shear strain. Similarly, due to its crystal symmetry, there are only five independent stiffness constants for wurtzite zinc oxide. The stress vector presented in equation 2.1 is thus given by [97]: 


$$
\left(\begin{array}{l}
\sigma_{11} \\
\sigma_{22} \\
\sigma_{33} \\
\sigma_{23} \\
\sigma_{31} \\
\sigma_{12}
\end{array}\right)=\left(\begin{array}{cccccc}
C_{11} & C_{12} & C_{13} & 0 & 0 & 0 \\
C_{12} & C_{11} & C_{13} & 0 & 0 & 0 \\
C_{13} & C_{13} & C_{33} & 0 & 0 & 0 \\
0 & 0 & 0 & C_{44} & 0 & 0 \\
0 & 0 & 0 & 0 & C_{44} & 0 \\
0 & 0 & 0 & 0 & 0 & \left(C_{11}-C_{12}\right) / 2
\end{array}\right)\left(\begin{array}{c}
\varepsilon_{11} \\
\varepsilon_{22} \\
\varepsilon_{33} \\
\varepsilon_{23} \\
\varepsilon_{31} \\
\varepsilon_{12}
\end{array}\right)
$$

Equation 2.11

Figure 2.5 shows a simplified illustration of the desired structure for our zinc oxide piezoelectric transducer, along with the expected electromechanical response $\left(d_{33}\right)$ along the c-axis due to stress along the same direction. This $\mathrm{ZnO}$ structure alignment maximizes the piezoelectric response along the c-axis direction; i.e., thickness mode, where this response would vary according to the applied stress.

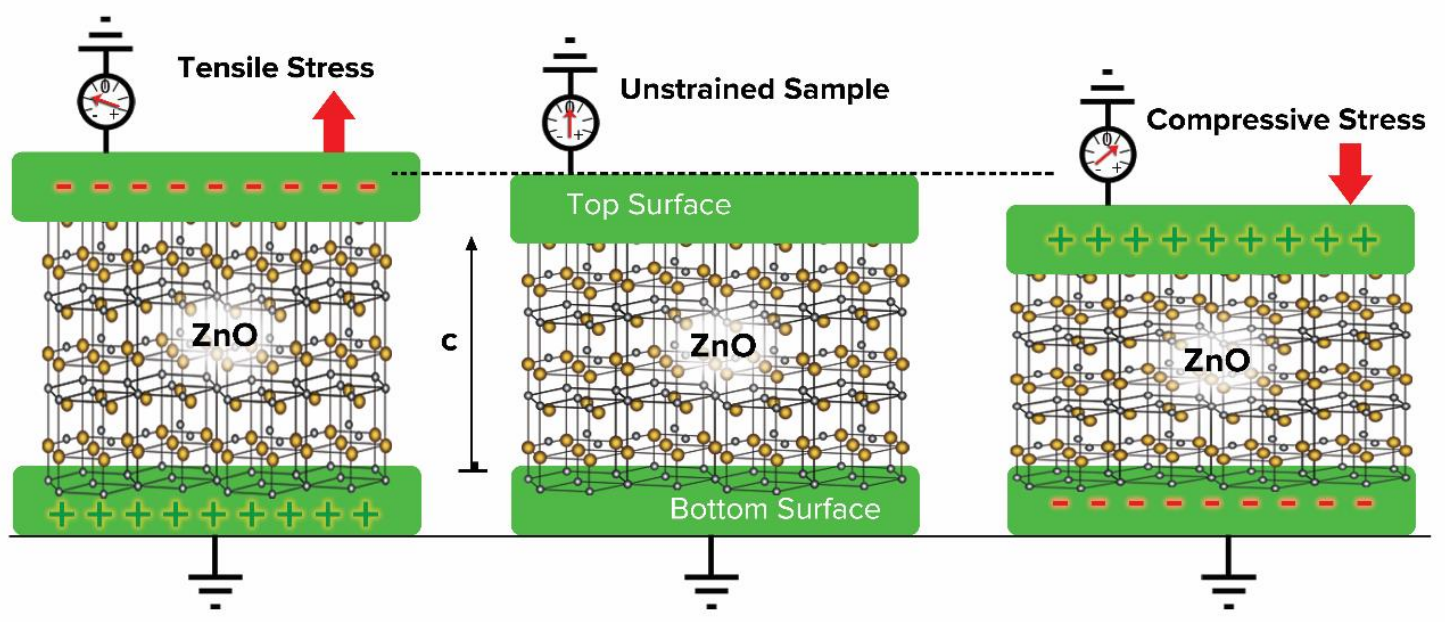

Figure 2.5: A schematic showing the desired c-axis oriented $\mathrm{ZnO}$ crystal between the top and bottom contacts of our device structure. 


\subsection{Status Quo of High-Frequency Ultrasound Transducers}

High-frequency single element piezoelectric transducers with frequencies exceeding $50 \mathrm{MHz}$ have been developed and used in ultrasound imaging systems since the early 1970s [9, 28, 114]; however, they can only focus an ultrasound beam at a fixed focal distance, and require mechanical beam steering. This means that the frequency dependent axial and lateral resolutions are only attainable within the transducer's focal depth range [11], whereas the frame rate is limited by the mechanical motion of the transducer. These problems can be avoided when transducer arrays are used, since arraying allows the electronic control of the focal depth and beam position, which enables imaging at higher resolutions and frame rates [11].

However, due to the delicate dimensional constraints and material properties of high-frequency piezoelectric transducers, there is still a lack of commercially available simple single-element transducers that can operate at frequencies higher than $200 \mathrm{MHz}$, as the involved fabrication and integration processes are complicated and costly, requiring skilled and well-equipped process engineers $[11,28,27,29]$.

In comparison, the fabrication of high-frequency transducing arrays presents further challenges, with array elements requiring a high-level of reproducibility in terms of electromechanical properties and structure, while ensuring minimal element-cross-talk levels. This requires the realization of very small kerfs, and places stringent demands on the electrical and acoustic matching [11]. The toll of such challenges is reflected by the lack of commercially available piezoelectric transducer arrays capable of operation beyond $100 \mathrm{MHz}[11,28]$. 
However, with the continuous advancement of MEMs microfabrication techniques, continuous efforts have been carried out to modify and tweak the conventional processing methods of piezoelectric materials, to push the frequency limit of the fabricated high-frequency transducer elements and arrays [11].

PVDF offers many advantages such as flexibility, low density, and low acoustic impedance (4MRayls) to facilitate matching to biological samples (acoustic impedance $\approx 1.5 \mathrm{MRayls}$ ), but it suffers from low capacitance [115] and low electromechanical coupling coefficient [11], which deem it of little use to ultrasound imaging applications. Nonetheless, PVDF is still relevant to other high-frequency ultrasound applications, with PVDF hydrophones operating at frequencies up to $100 \mathrm{MHz}$ being commercially available [116].

Conventional piezoelectric ceramics such as PZT offer a wide range of optimal electrical and electromechanical properties for high-frequency transduction applications, but suffer from having large grain sizes in the 5$10 \mu \mathrm{m}$ range. Thus, to prevent any unwanted lateral resonance, the relevant fabrication technique should be modified to ensure producing films with small and uniform grains [11]. The creation of high-frequency piezoelectric PZT films is also hindered by the inherent film cracking and the presence of voids within the ceramic's perovskite structure [117]. Therefore, film densification is required to allow the creation of functional high-frequency ultrasound transducers [11]. Numerous efforts were done to reduce the grain size in PZT sol-gel techniques, and ceramics having particle sizes in the 100-300nm range became available using composite sol-gel preparation methods [118, 119]. Accordingly, using solgel infiltration and pyrolysis, Dorey et al. [120] demonstrated the ability to densify PZT films through repetitive fabrication steps, to achieve crack-free films with a final thickness up to $10 \mu \mathrm{m}$. Such advancements were later put into 
use, along with the establishment of the aerosol [121] and hydrothermal [122] deposition techniques, allowing the reproducible creation of single PZT transducer elements capable of operation beyond $100 \mathrm{MHz}$ [123] and up to $200 \mathrm{MHz}[124,125]$.

Linear transducer arrays still rely on mechanical dicing to create kerfs to separate the array elements, where dicing was proven to be a viable process during the development of piezoceramics arrays operating at frequencies up to $35 \mathrm{MHz}[115,126]$. However, as the frequency of operation increases, the required kerfs become too small, and novel alternatives are sought. Recently, the realization of kerfless PZT linear arrays capable of operation up to $100 \mathrm{MHz}$ using composite sol-gel machining processes have been reported by several groups [127, 128]. However, despite the material suitability and the relative implementation success of high-frequency PZT transducers, the toxicity of lead poses a major threat to the environment, and governmental regulations continue to push towards the decrease and substitution of lead in applications [11]. Lead-free piezoelectric ceramic alternatives such as bismuth sodium titanate and (KNN)-based materials continue to be developed; however, they still suffer from poor electrical properties for practical device applications [11].

Concerning high-frequency $\mathrm{ZnO}$ transducers, MEMs fabrication techniques have recently shown great promise with regards to sophisticated device realization. MEMs fabrication technology relies on photolithography, etching, as well as $\mathrm{ZnO}$ deposition, with sputtering being the most common piezoelectric film deposition process, since it allows growing crystal oriented ZnO films with uniform thickness on a wide variety of substrates [11, 129].

Sputtered ZnO was used as early as 1995 to develop linear transducer arrays capable of operation at frequencies up to $100 \mathrm{MHz}$; however, such arrays suffered from having a large element pitch which made them of little use to 
medical imaging applications [45, 130]. Recent reports have emerged documenting the reliable production of simple and self-focused single piezoelectric $\mathrm{ZnO}$ elements capable of operation at frequencies exceeding $200 \mathrm{MHz}[27,3,11,131]$. $\mathrm{ZnO}$ based single element transducers capable of operation at $1 \mathrm{GHz}$ have also been reported; however, the output signal amplitude and performance of such transducers were found too low and impractical for acoustic microscopy purposes [5]. A summary of the recent work done on high-frequency $\mathrm{ZnO}$ piezoelectric transducers is provided in table 2.3.

Hence, given the simple structure and versatile properties of $\mathrm{ZnO}$ as well as the availability of various deposition and patterning techniques, and with further optimization, this material is a promising candidate to develop highfrequency ultrasound transducer elements and arrays capable of operation beyond $300 \mathrm{MHz}$. At such high frequencies, acoustic attenuation levels become very significant [5], forcing the focal point of a transducer to be located as close as possible to the transducer's surface, and making self-focused transducers the preferred structure of choice [11].

With the development of novel lenses and the optimization of thick piezoelectric film fabrication processes, it is expected that commercial transducer arrays capable of operation beyond $200 \mathrm{MHz}$ will be available in the near future [11]. Such commercial availability would allow the applications of high-frequency ultrasound transducers to expand towards the fields of cellular bioengineering and lab-on-chip devices [11]. 
Table 2.3: Summary of the recent results on high-frequency $\mathrm{ZnO}$ transducers

\begin{tabular}{|c|c|c|c|c|c|c|}
\hline Reference & Frequency & Sputtering method & Structure & ZnO Thickness & Substrate & Contact \\
\hline Ref. [30] & $100 \mathrm{MHz}$ & RF magnetron & 32-Element linear array & $10 \mu \mathrm{m}$ & (0001) Sapphire & $\mathrm{Cr} / \mathrm{Au} / \mathrm{Cr}$ \\
\hline Ref. [104] & $100 \mathrm{MHz}$ & $\mathrm{RF}$ & Single element & $18 \mu \mathrm{m}$ & Curved $\mathrm{Al}$ rod & $\mathrm{Al} \mathrm{rod}$ \\
\hline Ref. [125] & $215 \mathrm{MHz}$ & RF magnetron & Self-focused single element & $13 \mu \mathrm{m}$ & (100) Si & $\mathrm{Al}$ \\
\hline Ref. [80] & $50-130 \mathrm{MHz}$ & RF magnetron & Single & $25 \mu \mathrm{m}$ & Sapphire, fused silica & $\mathrm{Ni}$ \\
\hline Ref. [126] & & RF magnetron & Linear array & $8 \mu \mathrm{m}$ & $\mathrm{Si}$ & $\mathrm{Ti} / \mathrm{Pt}$ \\
\hline Ref. [127] & $204 \mathrm{MHz}$ & RF magnetron & Single element & $14.5 \mu \mathrm{m}$ & Curved Al rod & Al rod \\
\hline Ref. [128] & $112.5-186.5 \mathrm{MHz}$ & RF magnetron & Single element & $15-26 \mu \mathrm{m}$ & $\mathrm{Si} ; \mathrm{SiO}_{2} / \mathrm{Si}$ & $\mathrm{Al} ; \mathrm{Ti} / \mathrm{Pt}$ \\
\hline Ref. [12] & $1 \mathrm{GHz}$ & Reactive & Single element & $1.4 \mu \mathrm{m}$ & $(100) \mathrm{Si}$ & $\mathrm{Au}$ \\
\hline
\end{tabular}




\section{CHAPTER 3}

\section{Fabrication}

The realization of our high frequency zinc oxide transducer structure described in the previous chapters and shown in figure 3.1 relies on depositing the different constituent films on top of a substrate of choice.

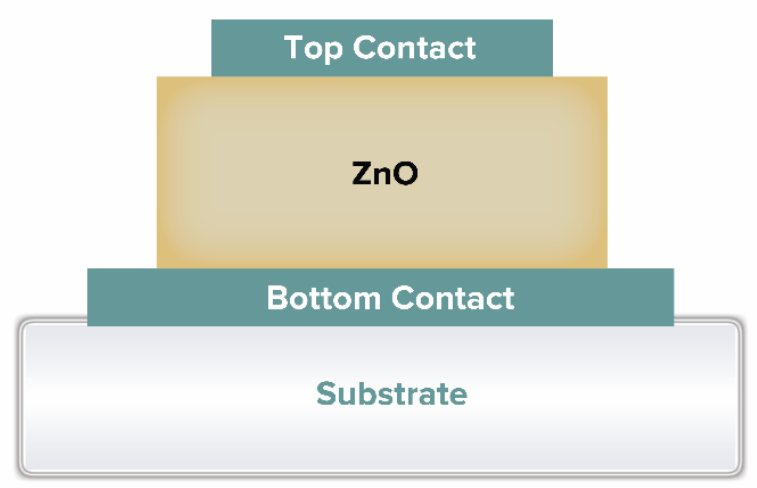

Figure 3.1: Schematic showing our simplified transducer structure. 
The chosen substrates were transparent, to facilitate our secondary objective for creating fully transparent transducers, as described in the first chapter. The constituting contact and active layers were deposited using physical vapour deposition techniques, namely by thermal evaporation and radio frequency magnetron sputtering. Thermal annealing inside and outside the sputtering chamber was applied to examine its effect on the fabricated material properties.

In the following parts of this chapter, the tools and materials used to create our transducer structures and samples are described.

\subsection{Substrate}

\subsubsection{Substrate materials}

In order to evaluate and compare the substrate effects on the grown zinc oxide film properties, three different transparent substrate materials were used throughout this work:

- Soda-lime glass, which is a cheap and relatively low-quality amorphous substrate material. At the first stages of this project, this substrate served as an economic alternative for evaluating and optimizing the $\mathrm{ZnO}$ deposition parameters.

- Borosilicate glass (borofloat 33), which offers a polished higher-quality amorphous wafer surface, with thermal shock resistance due to having a coefficient of linear thermal expansion as low as $a=3.25 \times 10^{-6} \mathrm{~K}^{-1}$. Wafers from University Wafers ${ }^{\circledR}$ having a 2-inch diameter, a $0.5 \mathrm{~mm}$ thickness, and a root mean squared roughness $\leq 1.3 \mathrm{~nm}$ were used. 
- C-plane oriented crystalline sapphire wafers, with a double-side polished finish. Wafers from University Wafers ${ }^{\circledR}$ having a 2-inch diameter, a $0.43 \mathrm{~mm}$ thickness, and a root mean squared roughness $\leq 0.55 \mathrm{~nm}$ were used.

When considering crystalline substrates, lattice mismatches between the deposited films and the substrate play a major role in defining the films' residual stress levels and dislocation density [31, 97]. Our choice for using cplane sapphire wafers was due to its popular use as a substrate material for $\mathrm{ZnO}$ heteroepitaxial growth on the basal plane (c-plane) orientation (0001) and on the a-plane orientation $(11 \overline{2} 0)[31,132]$; a popularity that can be attributed to the sapphire's relative low cost, wide energy bandgap, and availability in largearea wafer form [31].

Fons et al. [133], and Chen et al. [134] reported an epitaxial relation between the c-plane normals of grown $\mathrm{ZnO}$ films and sapphire wafers, as well as between the m-plane normal of a $\mathrm{ZnO}$ film and the a-plane normal of a sapphire wafer; such epitaxial alignment can be helpful for achieving a better selectivity for $\mathrm{ZnO}$ film growth along the c-axis orientation. Moreover, it was reported that a high degree of surface flatness was achieved for single-crystal $\mathrm{ZnO}$ films grown on top of sapphire wafers, which is an important property for our device structure fabrication.

\subsubsection{Substrate preparation}

In order to achieve the desired wafer shapes, a diamond tip scriber was used to cleave the crystalline sapphire wafers, whereas a dicing saw with a rotating diamond saw blade was used to cut the amorphous substrates. Solvent cleaning and ultrasonic baths were used to clean the wafers after dicing or scribing, to 
prevent contamination and improve the adhesion and uniformity of the deposited films. Lint-free wipes drenched in drum quality acetone were used to wipe the wafers surfaces; then, the wafers were dipped in an acetone bath. Subsequently, a nitrogen gas spray gun was used to dry the wafers, which were then sonicated at room temperature for several minutes in instrument grade isopropanol. Finally, the wafers were dried using the nitrogen gas spray gun, then placed on the sample holder relevant to the next processing step.

\subsection{Thin-film Fabrication}

Thin-films are primarily formed using wet processes like plating, chemical solvent deposition and chemical melt deposition, or dry processes such as physical vapour deposition and chemical vapour deposition.

Several techniques have been reported to produce high quality single crystal ZnO films, such as RF magnetron sputtering [135], molecular beam epitaxy [134, 133], pulsed laser deposition [136], hydride or halide vapour phase epitaxy [137, 138], and metal-organic chemical vapour deposition [139]. A comprehensive comparison of such techniques is found in reviews by Özgür and Triboulet $[31,108]$.

Since early investigations, sputtering techniques have been widely used to produce $\mathrm{ZnO}$ films, due to relative processing simplicity, low processing temperatures, low cost, good thickness uniformity, and relatively high deposition rates [31, 140, 141, 142]; therefore, RF magnetron sputtering was the chosen technique to create the high-quality piezoelectric $\mathrm{ZnO}$ films in this study. Different patterning alternatives were investigated to create the suitable masks for each deposited layer. This included masking by various kapton tapes, 
masking by laser-cut stainless steel sheets, and photolithography (more details in chapter 5).

\subsubsection{Physical vapour deposition}

Physical vapour deposition (PVD) techniques were used to create the metal contact thin-films (thermal evaporation) and the doped and undoped zinc oxide layers (RF magnetron sputtering). During such processes, source atoms are given enough energy to be transferred controllably from a purified source to a substrate, where film formation and growth proceed atomistically. Typically, this happens in a reduced pressure chamber, where the source atoms are brought into the gaseous vapour phase through physical mechanisms; mainly evaporation or collisional impact [24].

Soon after the relatively colder substrate is exposed to the incident vapour, a sufficient number of vapour atoms or molecules condense onto the substrate's surface. With a continuous supply of impinging atoms, the condensing atoms turn into a uniform distribution of small but highly mobile nucleation islands, which grow in size until island-merging (coalescence) occurs.

Crystallographic facets and orientations are usually preserved within an island (crystallite) and at interfaces between the coalesced particles. Coalescence continues until the islands are connected and the voids between them are filled, at that stage the film is said to be continuous; this typically happens when films are thicker than 5-10nm [24].

\subsubsection{Thin-film growth modes}

Three basic modes for film growth were identified by observation [24]: 
- When the deposited atoms or molecules are more strongly bound to each other than to the substrate, island (Volmer-Weber) based growth occurs, where the condensed cluster at each nucleation site grows in three dimensions, until a continuous film is achieved.

- Layer based growth (Frank-van der Merwe) dominate when the force binding the condensed atoms or molecules to the substrate is stronger than the force binding the atoms/molecules together. This growth mode is two dimensional, since planar sheets are consecutively grown on top of each other.

- An intermediate growth mode (Stranski-Krastanov) is a combination between the layer and island growth modes. This mode is essentially an unsustained layer growth mode, where the transition from sheet growth to three-dimensional growth is attributed to certain energy demanding/related factors, such as lattice mismatch, substrate temperature, and deposit atoms' energy.

\subsubsection{Structure zone model}

Irrespective of a deposited film's nucleation properties and growth mode, it was found that a film's surface and cross-sectional morphology will possess certain predictable features as growth continues beyond a certain film thickness (around 100nm [143]).

The earliest documentation of such a morphological model (structure zone model) was for evaporated metal and oxide films by Movchan and Demchishin in 1969 [144], where deposition parameters were reduced to as few as possible while illustrating their effects on the films' structure. In that case, it 
was found that the homologous temperature $T_{h}$ was the main parameter affecting the resulting film morphology.

The homologous temperature $T_{h}$ is defined as the normalized film growth temperature; i.e., substrate temperature, with respect to the deposited material's melting temperature $T_{m}$ :

$$
T_{h}=\frac{T_{s}}{T_{m}}
$$

Equation 3.1

$T_{h}$ is a dimensionless variable, whereas $T_{m}$ and $T_{s}$ are both expressed in Kelvin.

Movchan's and Demchishin's structure zone model (SZM) comprised three different morphological zones over the range of $T_{h}$. As sputtering became a popular film deposition technique, the structure zone model needed to accommodate a new parameter, the sputtering pressure of the inert gas, typically argon. This extended three-dimensional model is shown in figure 3.2, and was developed by J.A. Thornton in 1974, specifically for sputtered metal films [145].

According to this structure zone model theory, four basic phenomena are identified as responsible for determining a deposited film's morphology: shadowing, surface diffusion, bulk diffusion and desorption. Shadowing is attributed to the line of flight impingement of arriving atoms onto the rough surface of the deposited film, whereas diffusion and desorption rates directly scale with the melting temperature of the condensate [24].

The individual effects of shadowing, and bulk and surface diffusion are illustrated in figure 3.2, where the dominance of one or more of these processes is manifested by different structural morphologies. 


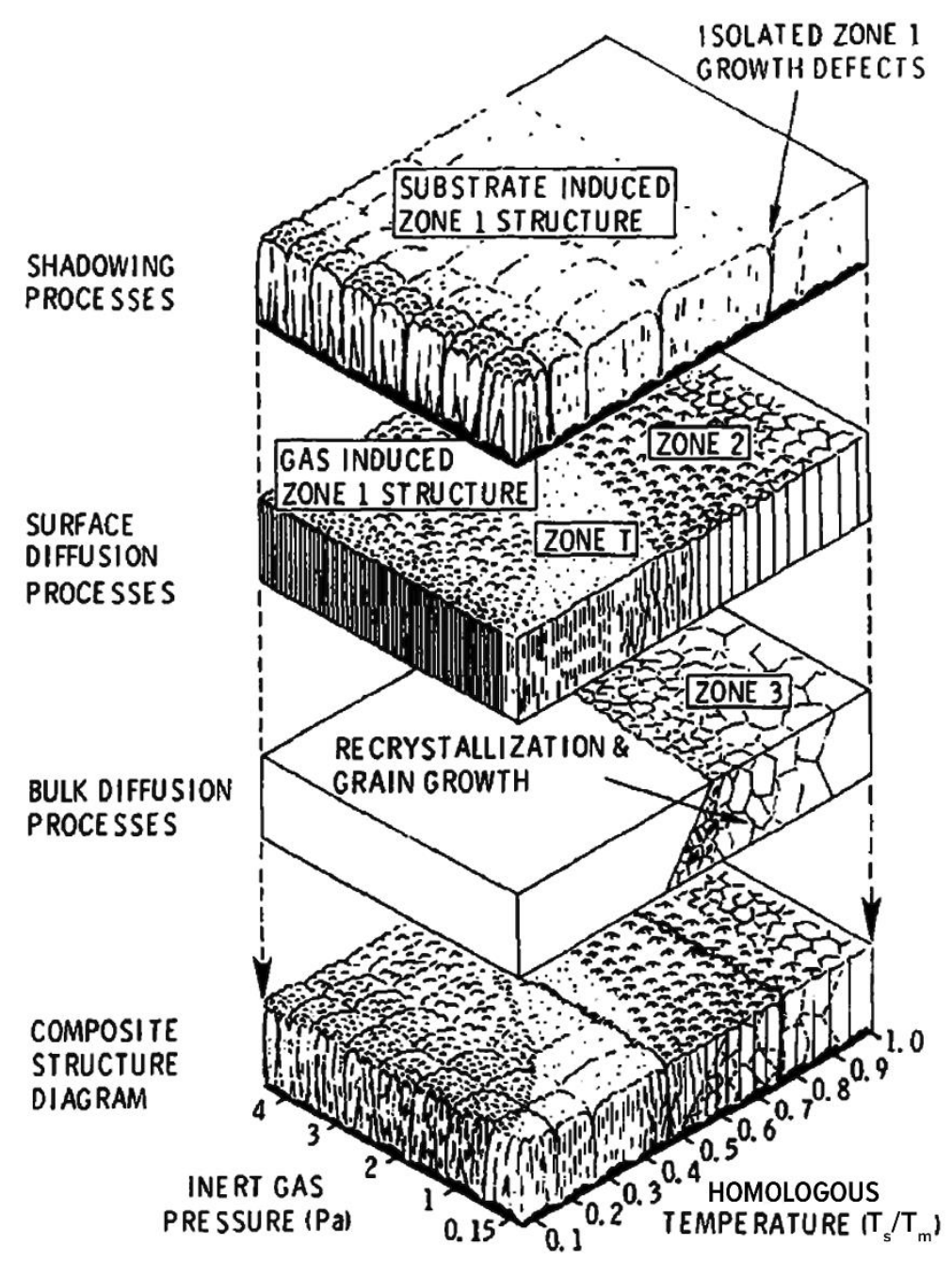

Figure 3.2: A schematic drawing showing the superposition of the physical processes, which contribute to a film's structural zones [24].

In addition to zones 1, 2, and 3, Thornton's extended model had an additional transition zone $(\mathrm{T})$, between the first and second zone. The main observations regarding the morphology of films grown in each zone are $[24,146]$ :

- Due to low substrate temperatures, films grown in zone 1 have low adatom mobility and are highly affected by shadowing; thus, they tend to have tapered and fibrous columns with dome shaped tops, voided 
grain boundaries, and high dislocation density. Such films are relatively harder if the condensate was a metal, while they tend to be relatively softer in the case of a ceramic.

- Films deposited in the transition regime have fibrous grains and dense grain boundaries, which translates to a smoother surface. The dislocation density is still high in this zone, and the resulting films have high strength and low ductility.

- In zone 2, surface diffusion is a dominant process during film growth; therefore, the grains are columnar and dense (granular epitaxy). Simply put, the condensate atoms in zone 2 have enough energy to allow surface diffusion within a given grain, but not enough energy to allow grain recrystallization; i.e., the available thermal energy is less than the grain surface energy.

- Zone 3 is characterized by lattice diffusion and low dislocation density, owing to the ample amounts of available energy. The grains tend to be large and non-columnar due to recrystallization. Contrary to zone 1, metal films grown in zone 3 are relatively soft, whereas ceramics are relatively harder when grown in this regime.

\subsubsection{Residual stress in films}

Deposited films are usually stressed even without the application of external loading [146], and are said to possess internal or residual stresses; such stresses can be classified as intrinsic or extrinsic. Extrinsic stresses are attributed to the boundary conditions of a deposited film, such as lattice mismatches with the neighbouring layers or substrate, or differences in thermal expansion properties as the condensate and the substrate cool down [146]. Intrinsic stresses on the 
other hand arise due to the internal structure of a deposited film, with main affecting factors including grain shape and size distribution, and void density. This implies that intrinsic stress is usually induced during film deposition, whereas extrinsic stresses can build up during deposition, as well as post deposition due to thermal effects.

The geometric effect of internal stresses during film deposition is illustrated in figure 3.3. Mechanical equilibrium dictates that the net force and bending moment vanish at the condensate-substrate cross-section, and compatibility requires that the covered substrate area equals that of the top of the deposited layer [24].

In the case when a film contains internal tensile stresses, the substrate experiences balancing compressive forces; this combination is not in mechanical equilibrium due to the uncompensated bending moment at the deposition edge. Thus, if the film-substrate pair movement was unrestricted, it will bend down; i.e., the substrate bends concavely upward, to counteract such bending moment. Similarly, a film possessing residual compressive stresses will cause the substrate to bend convexly outward [24].
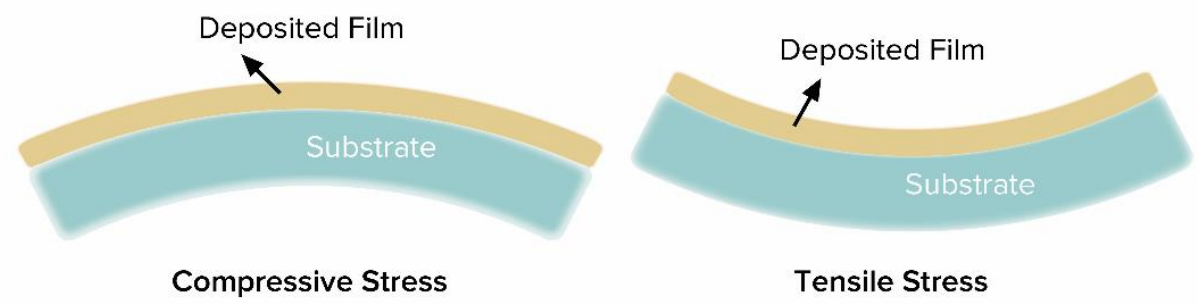

Figure 3.3: Deposited films can possess tensile or compressive residual stresses. For clarification, this schematic highly exaggerates the stress related effects on the shape of the filmsubstrate pair. 
Residual stresses affect the structural, mechanical, and electronic properties of a condensate, mostly in a negative and undesirable fashion, with large stresses typically resulting in film cracking and peeling off the substrate [147]. Depending on their material properties, stressed films will sometimes relax over time, which imply that the films' characteristics will keep changing until stresses become stable [148]. In some cases, however, it is favourable for a film to possess internal stresses, to counteract any predicted harmful forces during operation [24].

\subsubsection{Metal contact deposition}

Given its popularity [127, 31], gold was examined as a top and bottom metal contact material, where thin layers of chromium were used to improve adhesion between the bottom gold layer and the substrate, as well as between the gold layers and the zinc oxide film. Both metals were deposited using an Angstrom Engineering thermal evaporation system.

\subsubsection{Thermal evaporation}

During thermal evaporation, the source materials are placed on evaporation boats or are readily-plated on tungsten wires. The wafers are placed on a sample holder, and kept in place using kapton tape or metal holders.

Then, the chamber is pumped down to vacuum (around $2 \times 10^{-6}$ torr), usually overnight in our system. Thereafter, the boat/wire is heated using joule heating, which causes the source material to evaporate. Obviously, the heated boats or wires must reach the temperature of the evaporant in question while having a negligible vapour pressure to reduce contamination. The evaporated atoms are deposited onto the exposed surfaces; hence, a shutter keeps them away from the wafer until a certain chamber pressure and deposition rate are 
established. The deposition rate is typically monitored using a quartz crystal microbalance [149], and adjusted by varying the current passing through the evaporation source. As soon as the shutter is opened, the evaporating atoms impinge onto the wafer's surface, thereby creating a film as sketched in figure 3.4. Once the targeted film thickness is reached, the shutter is closed, then the current is shut down to stop the deposition process. The chamber is then vented back to atmospheric pressure using nitrogen gas.

A profilometer is then used to measure the deposited film thickness and to verify the tooling factor for that given material; i.e., the ratio of the measured deposited film thickness to the thickness monitor's reading.
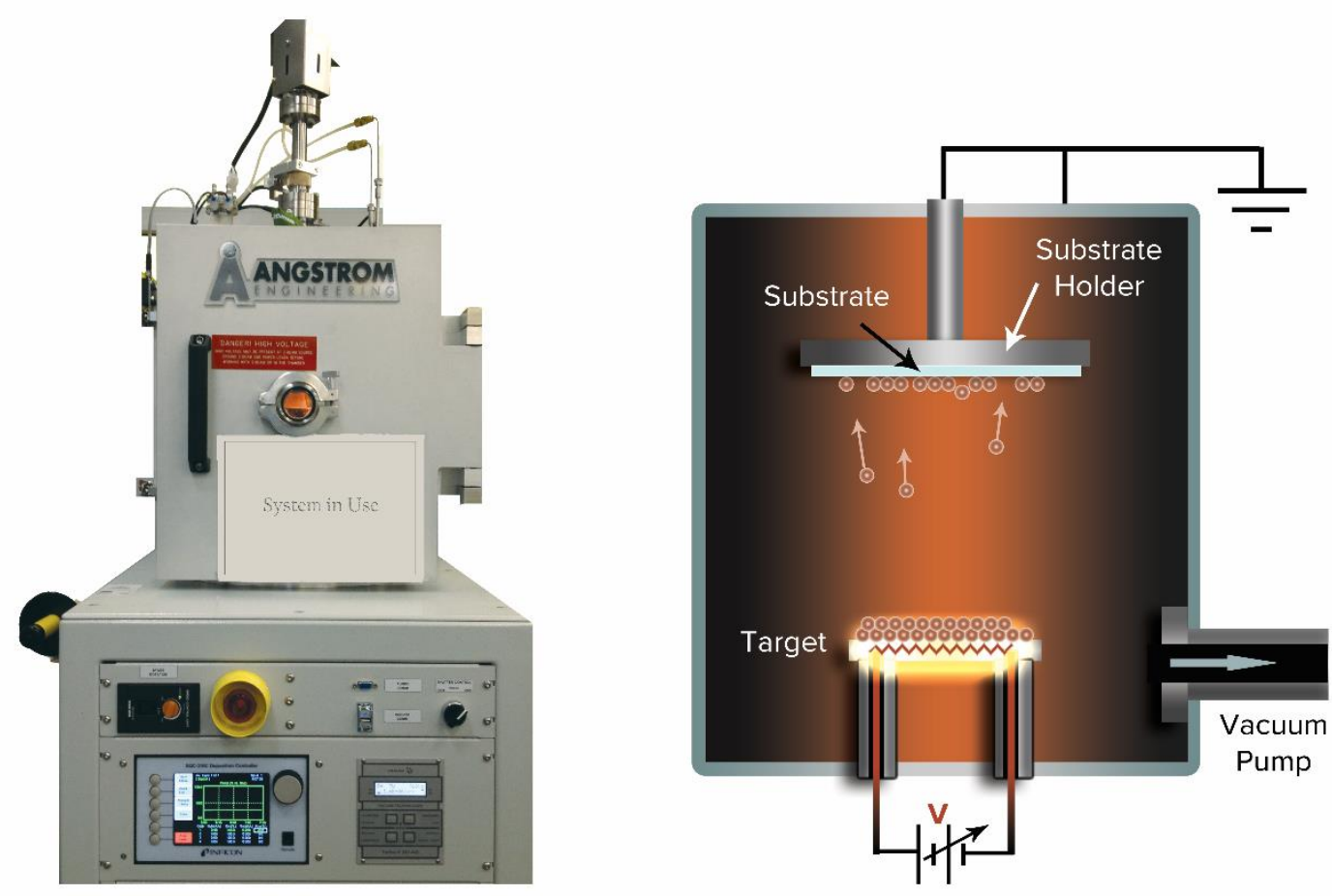

Figure 3.4: The thermal evaporation system used, with a simplified schematic of the evaporation chamber 


\subsubsection{Zinc oxide thin-film deposition}

\subsubsection{Top-down}

We have fabricated single-element PZT transducers at Callaghan Innovation in Lower-Hutt, New Zealand, in order to evaluate the upper limits for the topdown microfabrication techniques.

The polled PZT ceramic was purchased in a diced bulk rectangular form $\left(1 \times 1 \times 0.1 \mathrm{~cm}^{3}\right)$, attached to a $6 \mathrm{~mm}$ backing layer. First, the whole block was plated with $20 \mathrm{~nm} / 200 \mathrm{~nm}$ titanium/gold layers, to form the bottom electrical contact. Then, aluminium oxide powder was used to lap-down and polish the PZT block to the minimum possible thickness, which was defined as the thickness at which the whole PZT layer was visibly removed at one of the edges of the ceramic (due to the ceramic's uneven surface). The following step was the deposition of another titanium/gold contact to create the top contact, which was patterned using the relevant masking and etching techniques.

The measured thickness of the resulting PZT transducers was in the 30$60 \mu \mathrm{m}$ range, which indicated poor thickness control and reproducibility for this fabrication process.

\subsubsection{Sputtering}

Sputtering techniques have long been proven to produce high-quality, c-axis oriented and piezoelectric $\mathrm{ZnO}$ crystal films [43, 46, 150]; hence, sputtering was selected for depositing both the active zinc oxide films and the doped conductive zinc oxide contacts in this work.

During sputtering, ions are accelerated towards a target, causing the target atoms or molecules to be ejected by means of momentum transfer. The 
ejected atoms condense at the opposing surfaces, forming a film on a given substrate. Therefore, sputtering is a physical vapour deposition technique.

The target is a plate of the material from which the sputtered film is to be synthesized, and it is connected to the negative terminal (cathode) of a power supply.

Facing the target is the substrate, which can be grounded, biased positively or negatively, electrically floating, heated, cooled, rotated, or some combinations of these.

During a sputtering process, the system is pumped down to vacuum, typically around $1 \times 10^{-6}$ mbar, then an inert gas (typically argon) or a mixture of gases is introduced to the chamber, with partial pressures within the $10^{-3} \mathrm{mbar}$ to $10^{-2} \mathrm{mbar}$ range. The gas background serves as the medium through which the glow discharge is initiated and sustained.

Positive ions in the plasma discharge are accelerated towards the cathode, where they strike the target's surface atoms, and transfer a part of their energy/momentum throughout the process. When this energy/momentum transfer is large enough, it is possible to eject neutral target atoms towards the opposite chamber surfaces. A fraction of the ejected atoms eventually condenses on the substrate's surface, forming a deposited film.

The efficiency of a sputtering process is determined by measuring the sputter yield, which is defined as the number of ejected atoms or molecules from a target surface per incident ion.

For a composite-material target, different components usually have different vapour pressures and sputter at different yields. Nevertheless, the disparity in the sputter yield is usually smaller than that of the vapour pressure, which means that sputtering offers a better stoichiometry control than regular 
evaporation techniques [24]. In addition, the kinetics of sputtered atoms is different from evaporated atoms, as the average energy for sputtered atoms is much higher. Thus, for a given material, the properties of the deposited films will vary according to the deposition process [151].

Sputtering processes are broadly divided into four categories: DC, RF, magnetron, and reactive, with differences arising in the sputtering system complexity, sputtering rate, and possible target materials. Important variants within each category and even hybrids between the categories do exist. For the scope of this project, only DC, RF, and RF magnetron sputtering techniques are described below. An in-depth analysis and description of sputtering processes is provided in $[23,24,152,151]$.

\subsection{DC sputtering}

This simple sputtering technique relies on applying a static electric field between the target and the substrate; i.e., the cathode and anode, respectively. Free electrons inside the chamber will then be repelled by the target, and are accelerated towards the anode. Electrons quickly attain high velocities due to their low mass, causing them to collide with the surrounding neutral and relatively heavy argon atoms. These collisions result in the excitation of argon atoms, or even knocking-off extra electrons (ionization) to turn them into positively charged ions $\left(\mathrm{Ar}^{+}\right)$. The excitation-relaxation processes of argon atoms are accompanied by photon emission events, which are responsible for the characteristic discharge glow of plasma. The positively charged argon ions are then accelerated towards the target, where they strike its surface.

Depending on an argon ion's energy and angle of incidence, as well as the binding energy and mass of the target atoms, one or more of the following scenarios may occur after a collision event $[24,152,151]$ : 
- The impinging argon ion is reflected back.

- $\quad$ The argon ion pushes the target atoms into new lattice positions; thereby damaging the target surface.

- The argon ion loses enough kinetic energy to the target atoms, causing them to be ejected from the surface; i.e., sputtering.

- Secondary electrons are knocked off the target atoms.

The emission of secondary electrons from the target, as described in the last scenario, is essential to obtain a steady-state self-sustaining plasma for the sputtering process to take place.

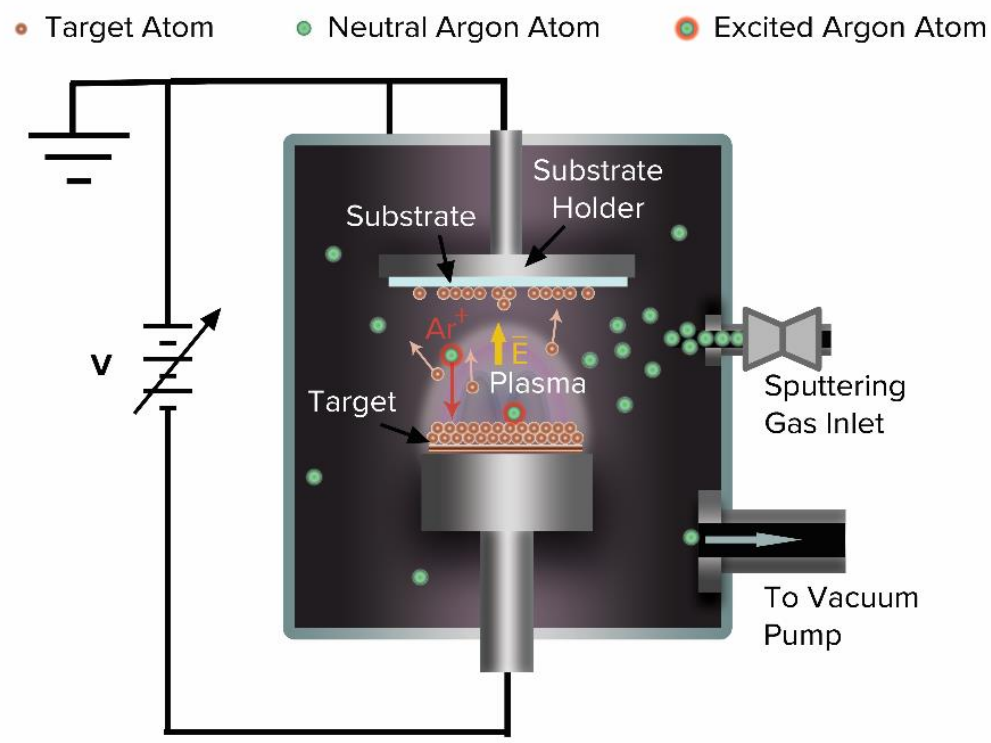

Figure 3.5: A simplified schematic of a DC sputtering system

Based on the arrangement shown in figure 3.5, it is evident that DC sputtering is unable to deposit semiconducting or insulating materials, as the target is required to be electrically conductive and to have a negative potential. Argon 
ions would simply positively-charge the surface of a non-conductive target, ultimately extinguishing the plasma.

\subsection{RF sputtering}

In order to deposit insulating, semiconducting, or high resistance target materials, radio frequency $(\mathrm{RF})$ sputtering is used. At frequencies higher than $50 \mathrm{kHz}[24]$, electrons in the glow discharge region have enough energy to cause ionizing collisions, reducing the need for secondary electron emission from target atoms to sustain the plasma discharge. In addition, at high-frequencies, an insulating target will conduct electricity in a capacitor-like fashion; thus, the applied RF voltage is coupled irrespective of the target material's impedance. The federal communications commission (FCC) has reserved the $13.56 \mathrm{MHz}$ frequency for RF plasma processing; hence, it is the most widely used frequency in RF sputtering systems [24].

Replacing the DC source with an RF source changes the way different plasma species react to the applied electric field, which explains why RF sputtering works. Essentially, even though an RF electric field lacks polarity, the target self-biases to a negative potential [24]. This is due to the fact that electrons are much more mobile than ions at such frequencies, making the discharge current-voltage characteristics similar to that of a leaky diode. Thus, as the RF signal is applied to the neutral target, a large electron current is drawn during the positive half-cycle, whereas a smaller amount of ions $\left(\mathrm{Ar}^{+}\right)$is drawn during the negative half cycle; i.e., net charge accumulation during an RF cycle is different from zero.

However, this mobility disparity applies at both contacts, which implies that both contacts should sputter. To circumvent this problem, the target must be an insulator that is capacitively coupled to the RF source. Then, the equivalent impedance across the sputtering contacts would resemble two 
capacitors connected in series, one at the substrate, and the other at the target sheath region. By having different areas for the sputter contacts, each of these two capacitors will have a different capacitance; thereby, the ratio of the voltage-drop through each capacitance will be far from unity. For a small capacitively-coupled target contact and a larger directly-coupled substrate contact, this voltage drop ratio is given by:

$$
\frac{V_{C}}{V_{d}}=\left(\frac{A_{d}}{A_{C}}\right)^{4}
$$

Equation 3.2

where $V_{C}$ is the voltage drop across the capacitance at the target contact, and $V_{d}$ is the voltage drop through the capacitance at the substrate contact; $A_{C}$ and $A_{d}$ are the respective contact areas.

In practice, the target has a small area in the order of several square inches, whereas the substrate contact includes all the other surfaces in the chamber (baseplates, chamber walls, etc.); this raises the target sheath potential while minimizing sputtering of the grounded chamber fixtures. Consequently, a steady state voltage distribution across the contacts will normally exist in an RF sputtering system.

\subsection{RF magnetron sputtering}

By placing magnets in the vicinity of the target, free electrons and secondary electrons emitted during sputtering will experience the well-known Lorentz force in addition to the electric field force. Using such forces, and by adjusting the magnetic field direction with respect to the electric field, it is possible to prolong the electron residence time in the plasma, effectively creating an electron trap around the target. This localized increase in the electron density 
improves the odds of ionizing argon atoms, which translates into enhanced sputtering rates. In an RF magnetron sputtering system, the magnets are typically placed underneath the target contact (figure 3.6).

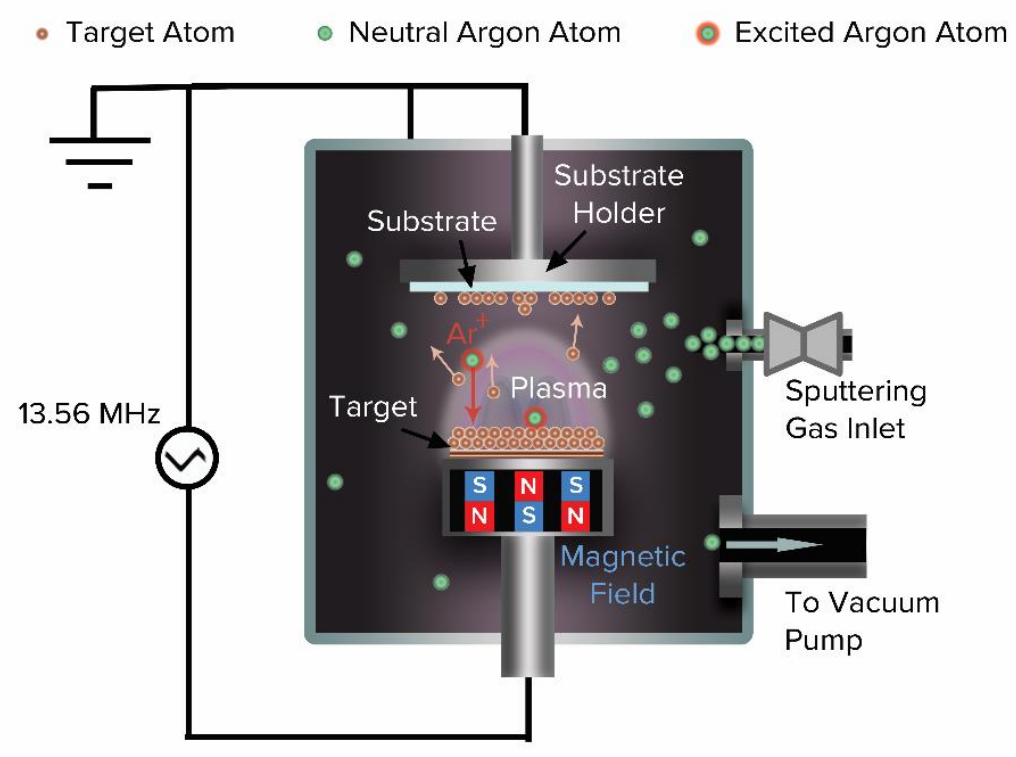

Figure 3.6: A simplified schematic of an RF magnetron sputtering system

\subsection{Sputtering set-up}

In this project, an Auto500 RF magnetron sputtering system from $H H V$ was used to deposit both doped and undoped zinc oxide films (figure 3.7). The system is equipped with three different target contacts, and has a single RF magnetron source. In addition, the system allows substrate rotation and heating. Several parameters could be adjusted to control the sputtering properties:

- RF power: a Dressler Cesar RF power supply was used to adjust the sputtering power between 0 and 600Watts.

- Sputtering and reactive gas flow rates; i.e., argon and oxygen, respectively. 
- Substrate temperature: a proportional integral derivative (PID) controller and a quartz heating lamp allowed temperature to be raised up to $350^{\circ} \mathrm{C}$, while a thermocouple was used for temperature monitoring.

- Substrate rotation: this could be applied at a constant 35rpm

- Sputtering pressure: this was regulated using the high vacuum valve.

- Substrate-to-target distance.

- Substrate-holder position with respect to the target.
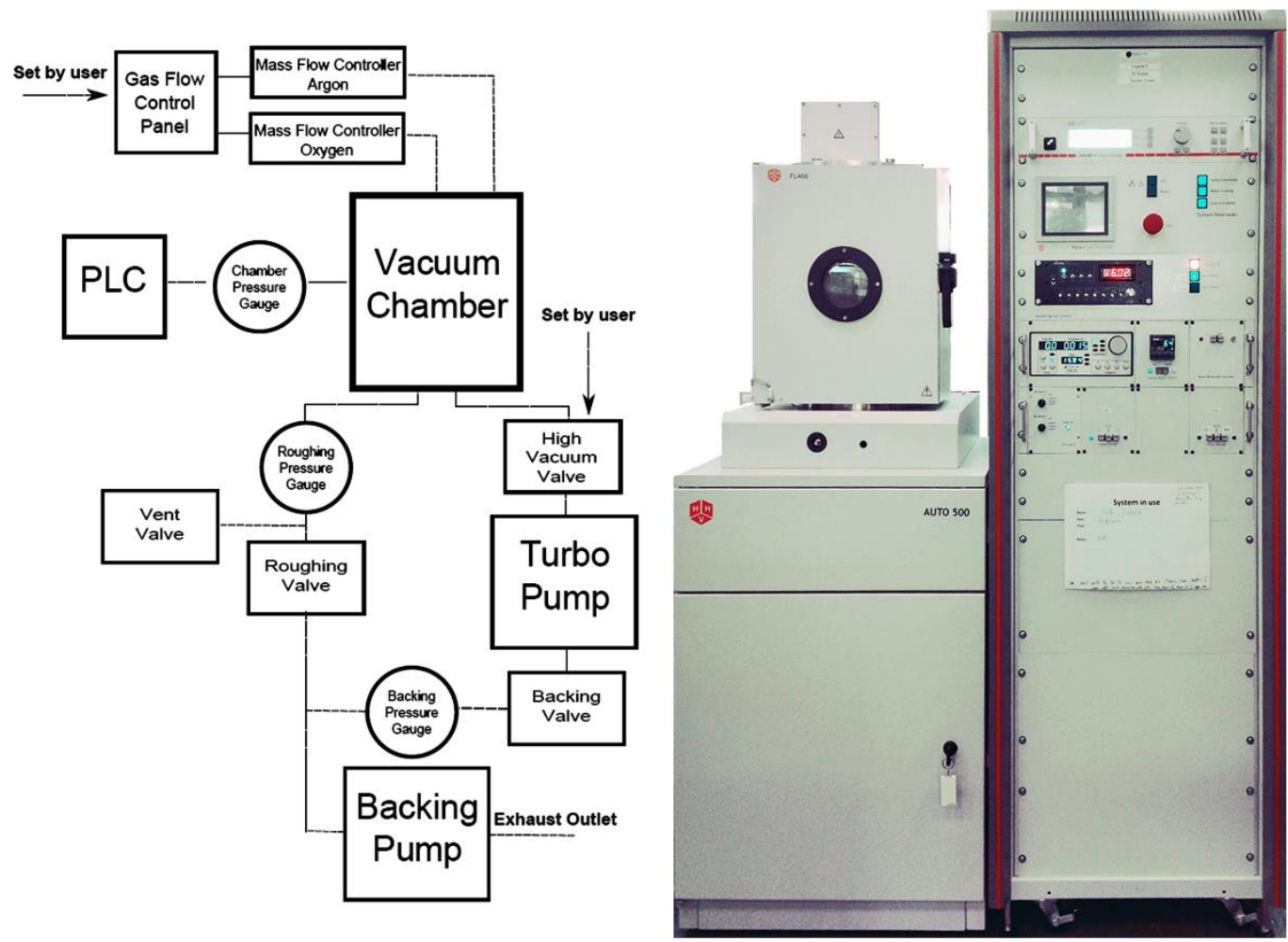

Figure 3.7: The Auto500 sputter coater system from HHV is able to support three different targets, and is fitted with an RF magnetron sputtering source. The main control panels are seen in the photo. The vacuum block diagram of the system is shown to the left. 
In addition, the system made it possible to deposit zinc oxide films using reactive sputtering, where a chemical reaction between the target material (zinc or zinc oxide) and a reactive gas (oxygen) could take place. Thus, film composition and stoichiometry could be controlled by adjusting the gas ratio (inert to reactive) while sputtering.

\subsection{Thermal Annealing}

Since RF magnetron sputtering is used to deposit our $\mathrm{ZnO}$ films, it is expected that the resulting films would have inherent compressive stresses due to the bombardment of energetic particles [11]. Therefore, annealing processes are carried out in this work to relieve the residual stresses in the deposited films. In addition, literature indicates that thermal annealing helps improve the electrical properties of piezoelectric films by prompting grain growth and improving the films' crystallinity $[153,26,154]$.

Our first annealing process is referred to as an in situ annealing step, and it is carried out inside the sputter coater chamber during the sputtering session, eliminating the need to break vacuum. A substrate quartz lamp heater is used for that purpose, where the maximum allowed substrate temperature was $350^{\circ} \mathrm{C}$ to prevent any chamber damage. The background gas mixture could be altered based on our needs, by controlling the argon and oxygen flow rates and partial pressures. This annealing step was used to reduce the residual stress, improve the crystal orientation, and increase the crystallites sizes of the zinc oxide seed layer.

The other annealing process is referred to as an external or post-growth annealing step, where the samples are placed in a quartz tube inside an 
insulated furnace, as shown in figure 3.8. The tube can be safely heated up to $1200^{\circ} \mathrm{C}$ for brief amounts of time; however a maximum annealing temperature of $1000^{\circ} \mathrm{C}$ was used in this study. The gas background inside the quartz tube could be altered during annealing, where a 95\%:5\% Ar: $\mathrm{O}_{2}$ mixture was used in our case. A PID controller is used to keep the furnace temperature close to the selected set-point during the annealing process. The furnace controller allowed us to set-up the annealing time and temperature, and the parameters of the temperature ramping events. Once the annealing process was complete, the furnace was allowed to slowly cool down to room temperature, as thermal shock can lead to film cracking due to thermal expansion mismatches between the wafer, the metal contact layers, and the zinc oxide film.

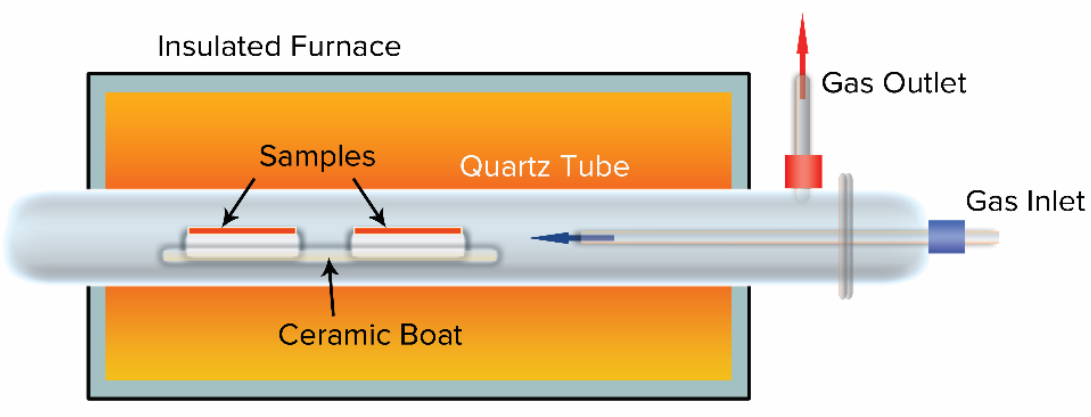

Figure 3.8: A schematic showing the external annealing setup used in this project 


\section{CHAPTER 4}

\section{Characterization Techniques}

Several characterization methods were used to identify the structural, electronic, and optical properties of the developed samples. Detailed and precise characterization was key for providing feedback and proof during the optimization of the relevant microfabrication processes. In this chapter, an introduction to the main characterization techniques and equipment used for this project is presented.

\subsection{Surface Profiler}

A Veeco Dektak 150 surface profiler [155] was used to measure the thickness of our thin-films using contact stylus profilometry. In this technique (figure 4.1), the substrate is placed on a flat sample stage that is equipped with suction capability to keep the sample in place. A sharp diamond-tipped stylus is then lowered onto the sample's surface, until contact is achieved. The stylus is 
attached to a flexible cantilever that senses and regulates the pressure applied by the tip onto the sample's surface. By laterally moving the stylus with respect to the stage, the cantilever keeps the tip in contact and detects any topographic variations relative to the initial baseline.

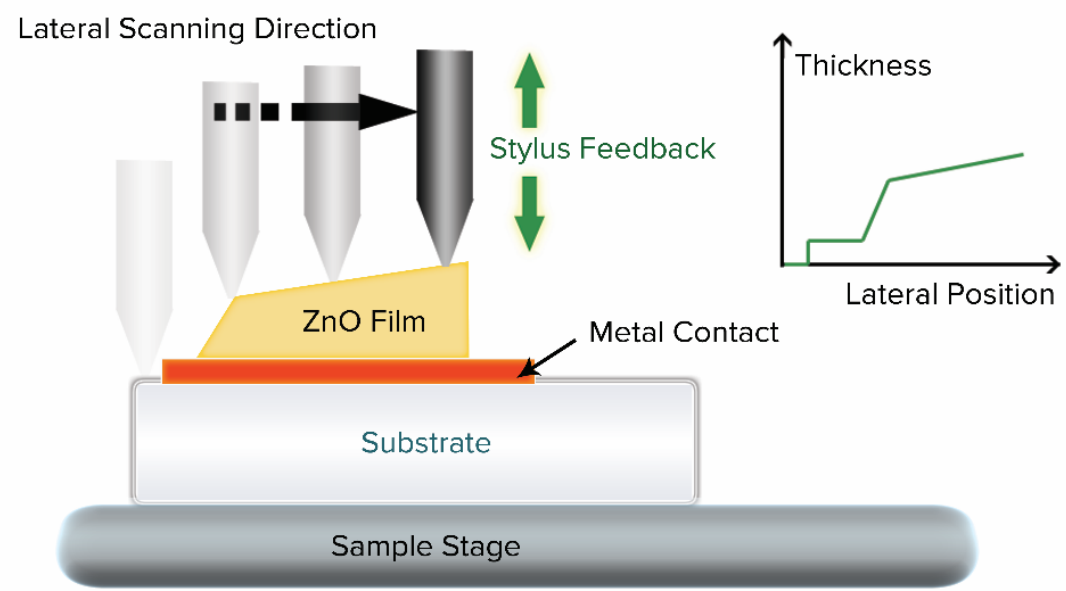

Figure 4.1: A schematic illustrating the operation of the Dektak profiler. Thickness variations on a flat substrate are directly measured by mapping the stylus's vertical position versus its lateral position with respect to the stage.

This technique provides a quick and reliable method for measuring a thin-film's relative thickness, surface roughness, and waviness. Furthermore, a crude estimate of a film's stress can be obtained by measuring the substrate's bow/curvature over large areas.

Although the system is capable of producing automated twodimensional profile plots, we only used it in the manual mode for thickness measurements across arbitrary cross-sections of our films. In order to minimize the surface damage due to tip contact, our stylus was equipped with a lowinertia sensor that kept the applied contact force at a constant preselected level 
between $1 \mathrm{mg}$ and $15 \mathrm{mg}$. Thickness variations up to $524 \mu \mathrm{m}$ could be measured with an acceptable vertical resolution [155]. The stylus's lateral speed was selected by specifying the scanning duration (between $3 \mathrm{~s}$ and 218s). The profilometer takes 300 data-points per second; hence, the lateral resolution was specified by adjusting the scanning duration and range. The maximum number of data points in a given scanning trace is 120,000. For our purposes, one shortcoming of contact stylus profilometry is the need of a baseline/step to carry out thickness measurements. This meant that reliable thickness measurements could only be performed near the edges of a film.

\subsection{XRD}

\subsubsection{Theory}

An x-ray diffraction (XRD) system from the X'Pert Pro series by PANalytical was used to characterize the crystal properties such as the available orientations, crystallite domain sizes, and residual stresses of our thin-films. X-rays are electromagnetic waves having a wavelength between $0.01 \AA$ and $10 \mathrm{~nm}$ (figure 4.2 ), thus occupying the region between gamma and ultraviolet rays in the electromagnetic spectrum [156].

They were discovered in 1895 by the German Professor Wilhelm Conrad Röntgen of the University of Würzburg [157], who realized that despite being invisible, those rays travelled in straight lines and affected photographic paper in a fashion similar to that of light. Röntgen could not understand the nature of the radiation, hence naming it an X-radiation; however, he soon realized that it can penetrate objects that are opaque to light, and made an "x-ray picture" of his wife's hand only two weeks after his discovery [158]. 
Despite the lack of proper understanding, $x$-rays instantly became a popular tool for physicians and engineers, who wanted to study the internal structures of opaque objects, and Röntgen's discovery landed him the first Nobel Prize in physics in 1901 [156].

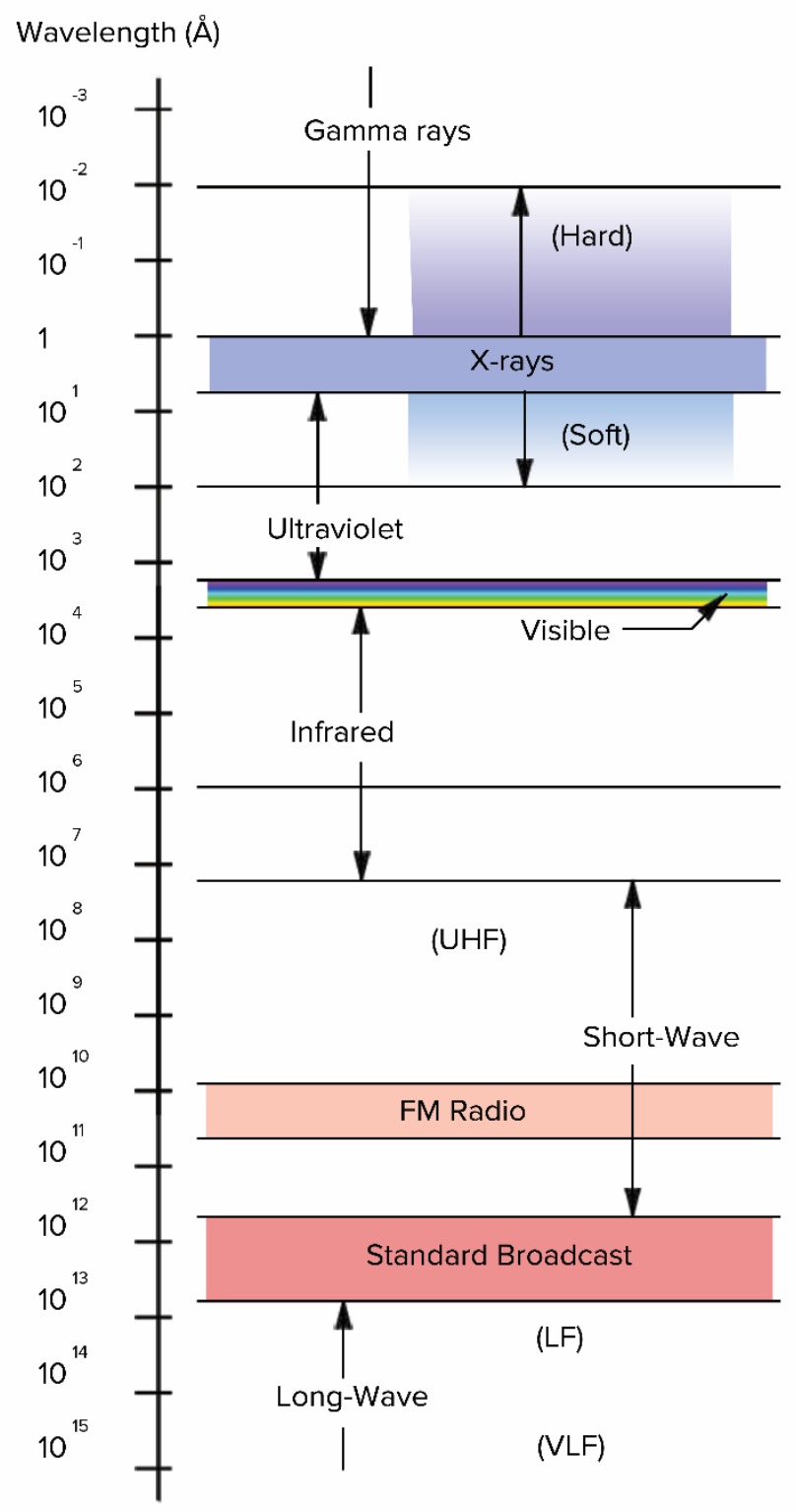

Figure 4.2: The electromagnetic spectrum 
By 1912, the phenomenon of x-ray diffraction by crystals was discovered [159], proving the wave nature of x-rays, and providing a method for investigating the atomic structure of matter.

X-rays are produced when an electrically charged particle rapidly decelerates, with the difference in the particle's kinetic energy being enough to yield an x-ray photon

$$
q V \geq \frac{1}{2} m\left(v_{i}^{2}-v_{f}^{2}\right) \geq h f=h \frac{c}{\lambda}
$$

Equation 4.1

where $q$ is the particle's charge, $V$ is the voltage required to accelerate the stationary particle to the initial speed $v_{i}, m$ is the particle's mass, and $v_{f}$ is the particle's speed after deceleration, $h$ is Planck's constant, $f$ is the emitted photon's frequency, $c$ is the speed of light, and $\lambda$ is the emitted photon's wavelength.

Typically, x-rays are produced in an x-ray tube (figure 4.3), where a current of electrons is accelerated between a cathode and a target anode, allowing the electrons to strike the target at very high speeds. Most of the absorbed kinetic energy is converted into heat, and less than $1 \%$ is transformed into x-rays [156].

The emitted x-radiation is polychromatic, since it is composed of photons having many different wavelengths, depending on the scattering events leading to the x-ray emission. This polychromatic radiation is usually referred to as white radiation, or Bremsstrahlung -German for breaking radiation, since it is due to electrons' deceleration. 


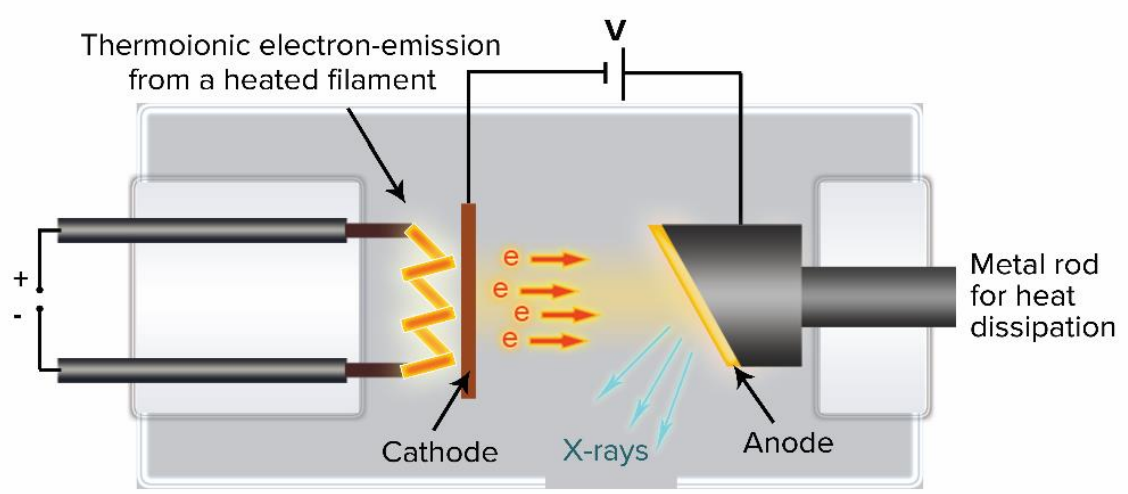

Figure 4.3: An X-ray tube schematic showing how electrons are accelerated by a voltage $\mathrm{V}$ towards the target anode, where they will scatter causing the generation of X-ray photons

If the energy of the accelerated electrons is higher than a certain threshold value -which depends on the target's material of choice- characteristic radiation is emitted and is superimposed on top of the white radiation (figure 4.4). Characteristic radiation occurs due to the ejection of electrons from the inner shells of the target's atoms, allowing the transition of higher energy-level electrons to fill the vacancies and give photons in return. Thus, this radiation is composed of discrete peaks that are positioned at the characteristic wavelengths $\lambda_{\text {char }}$ corresponding to the energy gaps $\Delta E$ between the two orbitals where transition occurs:

$$
\lambda_{\text {char }}=h \frac{c}{\Delta E}
$$

Equation 4.2

When the two orbitals involved in the transition are adjacent in energy, the characteristic line is classified as $\alpha$. However, when the transition occurs over a distance of two shells, the line is called $\beta$. The characteristic lines are 
categorized into several sets, referred to as $\mathrm{K}, \mathrm{L}, \mathrm{M}$, etc., according to the photon wavelength. This designation corresponds to the principal quantum number $n=1,2,3$, etc. in an atom.

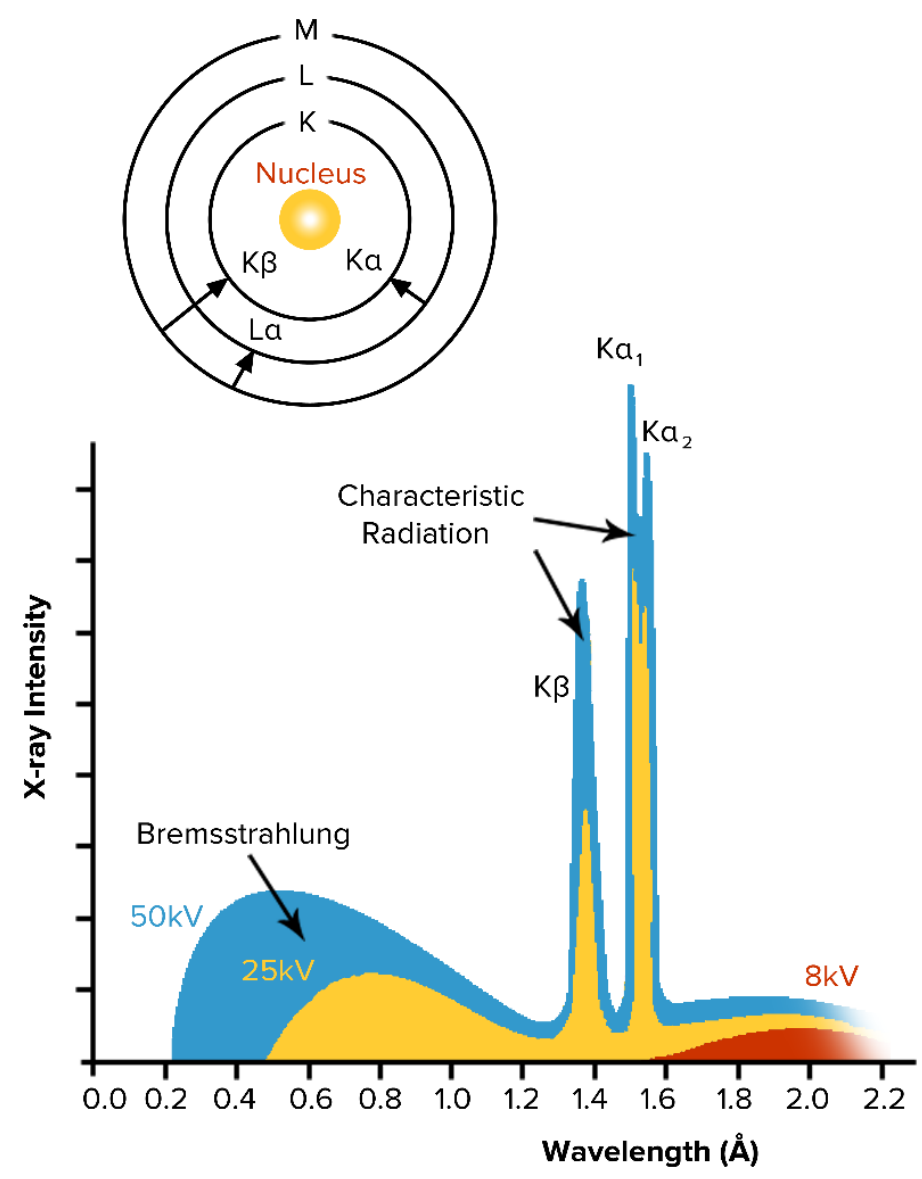

Figure 4.4: Top schematic shows the most common electronic transitions in an atom. The bottom diagram shows the typical emitted x-ray spectrum from a copper target. Notice that the characteristic radiation does not occur unless the accelerating voltage was beyond a certain threshold. Typically, the $\mathrm{K} \alpha$ lines are stronger than the $\mathrm{K} \beta$ lines, as it is more probable for a vacancy to be filled by an electron from an adjacent shell. 
For example, if an accelerated electron hits a target atom with sufficient kinetic energy, it can knock an electron out of the atom's K-shell, leaving the atom in a high energy-state. Consequently, an electron from a higher shell immediately falls into the K-vacancy, emitting a photon in the process; i.e., the characteristic $\mathrm{K}$ radiation in this case, and allowing the atom to go back to its ground energystate.

For x-ray diffraction (XRD) purposes, hard x-rays with wavelengths in the $0.5-2.5 \AA$ range are used [156], which are comparable to atomic distances, and where less-energetic photons are easily absorbed by a typical sample. In a conventional diffraction work, only the three strongest $\mathrm{K}$ lines are observed, as the $K \alpha$ line intensity is approximately two orders of magnitudes larger than the white radiation, and five times larger than the $K \beta$ line. For a copper target, the three dominant characteristic lines approximately occur at the following wavelengths [156]:

$$
\begin{aligned}
& K \alpha_{1}=1.54056 \AA \\
& K \alpha_{2}=1.54439 \AA \\
& K \beta=1.39222 \AA
\end{aligned}
$$

Usually, an XRD system would be optimized around the $K \alpha$ radiation, where filters and high resolution monochromators can be used to further filter out the $K \beta$ and $K \alpha_{2}$ spectral lines, if a $K \alpha_{1}$ monochromatic x-ray source was required.

X-rays used in diffraction are particularly harmful to living tissue, as they are easily absorbed by the exposed organs. However, since their wavelengths are comparable to the interatomic distances in solids, it is possible to qualitatively and quantitatively characterize a sample's crystal structure and 
chemical composition using diffraction. For that purpose, XRD is considered to be a non-destructive characterization technique for bulk, thin-film, and powder samples.

In an XRD system, a highly collimated and coherent $\mathrm{x}$-ray beam is directed at an angle $\theta$ onto the flat sample's surface. Incident photons will be scattered by the sample's atoms, primarily through the atoms' electrons.

When the energy of an incident photon $E=h f$ is comparable to the rest energy of the charged target particle $E=m c^{2}$, Compton scattering is assumed [160]. In such a quantum mechanical scattering, the incident photon loses some of its energy causing the charged particle to recoil; hence the reflected photon will have a longer wavelength due to the Compton shift.

However, if the incident photon is too cold; i.e., having a low frequency, it will not be able to relativistically interact with the charged particle. Therefore, a classical Thomson scattering takes place [161]. In this case, the charged particle experiences a force due to the incident photon's electromagnetic field, mostly due to the applied electric field. As the particle accelerates, it emits a photon of a similar frequency in return; hence, the x-ray wave is scattered "elastically".

Considering a monochromatic copper x-ray source for example, it will emit $K \alpha_{1}$ photons with an energy $E_{K \alpha_{1}}$ :

$$
E_{K \alpha_{1}}=h f=h \frac{c}{\lambda}=4.13566 \times 10^{-15} \times \frac{2.99 \times 10^{8}}{1.54056 \times 10^{-10}}=8.026 \times 10^{3} \mathrm{eV}
$$

An electron's rest energy $E_{m_{e}}$ is given by:

$$
E_{m_{e}}=m_{e} c^{2}=\frac{9.10938 \times 10^{-31} \times\left(2.99 \times 10^{8}\right)^{2}}{1.602 \times 10^{-19}}=511 \times 10^{3} \mathrm{eV}
$$


As the x-ray photon energy is much smaller than that of an electron's rest mass, which is four orders of magnitude lower than a proton's rest mass, x-ray diffraction can be described through a Thomson scattering model.

When an x-ray beam impinges onto a crystal's surface, two scattering modes are distinguished:

1. If path difference due to the scattering events is a wavelength multiple, the rays will be in phase and will reinforce one another. This constructive interference results in a diffracted beam having the same incidence angle. These diffracted beams are sometimes referred to as reflections.

2. If scattering occurs in any other direction, the scattered rays are out of phase and destructive interference results.

The condition required for achieving constructive interference due to multiple reflections from a crystal's parallel lattice planes (figure 4.5) was formulated by W. L. Bragg in 1913 [162]:

$$
n \lambda=2 d_{h k l} \sin \theta
$$

Equation 4.3

where $n$ is an integer specifying the order of diffraction, $\lambda$ is the photon's wavelength, $d_{h k l}$ is the lattice distance between the parallel diffracting crystal planes having the lattice planes with the (hkl) Miller indices, and $\theta$ is the angle formed between the incident beam's wave-vector and the diffracting lattice planes.

Thus, for a given monochromatic x-ray source with a fixed $\lambda$, and by varying the angle of incidence $\theta$, it is possible to measure a crystal's lattice spacings $d_{h k l}$, using an x-ray detector placed at an angle $(\pi-\theta)$ with respect to 
the same crystal surface reference, since intense diffraction will only occur at the specific angles satisfying Bragg's law.

In particular, this technique is used to study the composition of both crystalline and noncrystalline materials, and to evaluate the crystal properties of a solid sample [163]; available orientations, crystallite domains sizes, stress, texture, etc. An instrument which performs such characterization technique is aptly named a diffractometer [164].

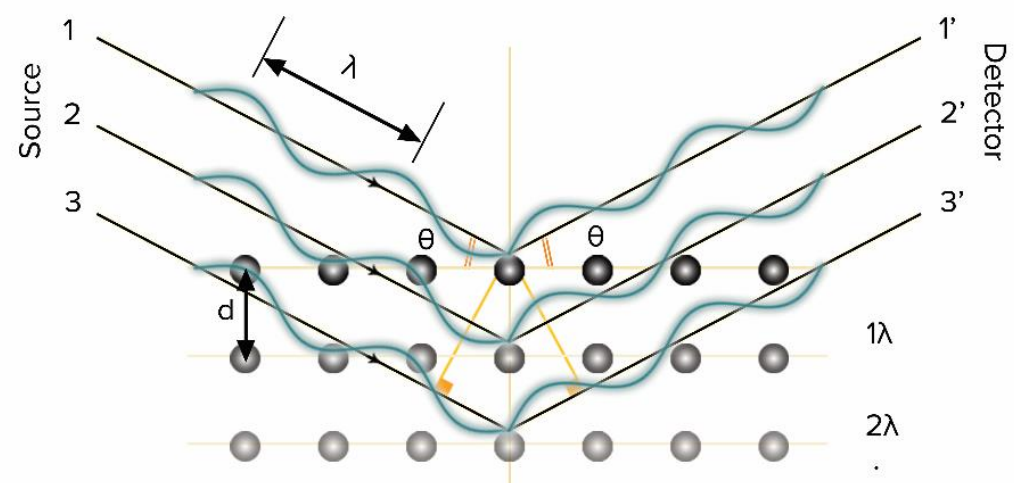

Figure 4.5: X-rays scattering of a crystal structure, causing bright diffraction spots (reflections) to be detected at the $\theta$ angles which satisfy Bragg's law

Although the diffracted beam intensity is significantly reinforced by the constructive interference due the crystal planes [156], it is extremely weak when compared to the incident beam, since only a small fraction of the $\mathrm{x}$-rays is scattered by the sample's atoms.

The intensity of a scattered x-ray beam $\left(I_{s}\right)$ by a single electron at a distance $r$ was found by Thomson [165] to be: 


$$
I_{S}=7.94 \times 10^{-30} \times \frac{I_{i}}{r^{2}}\left(\frac{1+(\cos 2 \theta)^{2}}{2}\right)
$$

Equation 4.4

where $I_{i}$ is the intensity of the incident x-ray beam. It should be noted that total beam reflection can occur when small angles of incidence are considered, below $1^{\circ}$ [156], where this x-ray reflectivity phenomenon does not follow the same scattering model, and is useful for studying surfaces and shallow internal interfaces in samples.

\subsubsection{X-ray diffractometer system}

The x-ray diffractometer system used in this study is an X'Pert Powder from the X'Pert Pro series by PANalytical [166]. Since our samples consisted of thin-films on top of flat substrates, we used the flat sample stage during our measurements. The x-ray source had a copper anode, where the accelerating voltage was set at $45 \mathrm{kV}$ with a constant current of $40 \mathrm{~mA}$. A $1^{\circ}$ anti-scattering slit was used for the incident beam, and a $10 \mathrm{~mm}$ wide divergence slit was used to restrict the width of the incident beam.

In a typical measurement, the sample is clamped to the flat and fixed sample holder, and a $(\theta-2 \theta)$ scan is carried out to generate the $\mathrm{x}$-ray diffractogram (figure 4.6). In such a scan, both the x-ray source and detector move while making an angle $\theta$ with the sample's surface; hence, their movement is symmetric with respect to a plane normal to the sample's surface, along the diffractometer circle. Accordingly, the resulting curve shows the diffracted beam intensity (arbitrary counts) versus $2 \theta$ (deg). 
Due to non-idealities, peak broadening results, where a given diffraction peak at an angle of $2 \theta$ will be measured as a broad packet having a full-width at half-maximum of $B(\mathrm{deg})$.
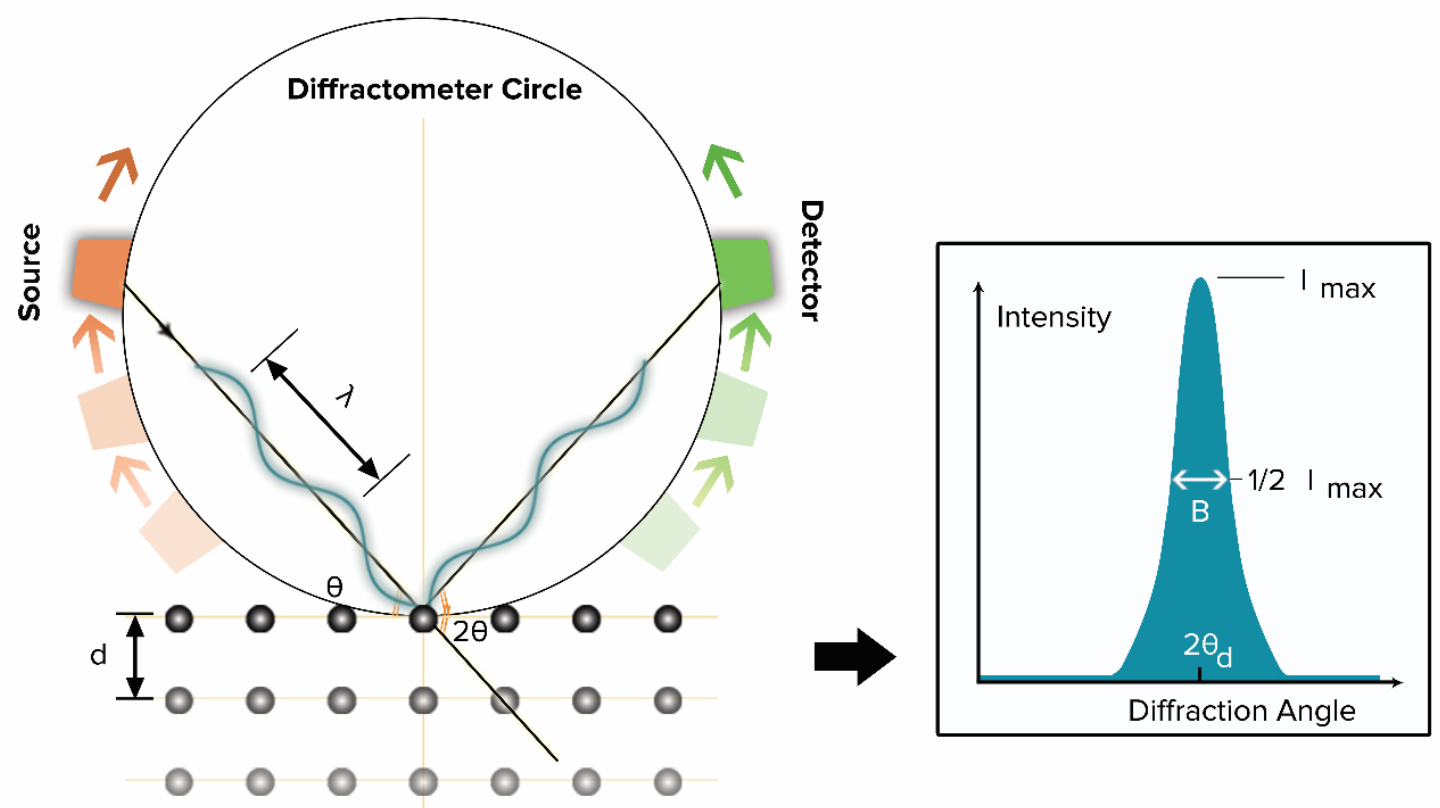

Figure 4.6: Left- In a $(\theta-2 \theta)$ diffractogram, the sample is kept stationary, while the source and the detector are symmetrically rotated along the diffractometer's circular path. Right- For a given crystal orientation, one peak will be detected at the incident angle satisfying Bragg's Law.

For the X'pert Powder system, the goniometer's minimum step size is $0.001^{\circ}$, the $2 \theta$ value can be varied between $-40^{\circ}$ and $220^{\circ}$, and the diffractometer's circle has a radius of $240 \mathrm{~mm}$ [166].

\subsubsection{Sources of errors}

While characterizing a solid specimen, several sources of systematic errors usually reduce the accuracy of diffractometers [167, 168]: 
- Instrument misalignment, in particular, if the source, detector, and sample are not on the focusing diffractometer circle. In addition, the centre of the incident beam should hit the sample's surface at a point belonging to the diffractometer circle.

- If the divergence slit used for the incident beam was not narrow enough, a broad irradiation width will result on the studied sample. This means that the sample's surface should be curved along the focusing circle to ensure accurate results.

- Sample transparency error results because not all x-rays are scattering from the same location, which causes peak position errors and peak asymmetry, with broadening towards the low $2 \theta$ angles. This error can be reduced by making the low absorbing parts of a specimen as thin as possible.

- When the sample's surface is off the focusing circle, the scattered beam does not converge at the correct position for the detector to pick up, as shown in figure 4.7.

Sample displacement error is usually the largest source of error in a diffractogram, and will result in incorrect peak positions. The error $\Delta d_{h k l}$ in the measured d-spacings of such a sample is given by [167]:

$$
\frac{\Delta d_{h k l}}{d_{h k l}}=-\frac{D \times(\cos \theta)^{2}}{R \times \sin \theta}
$$

Equation 4.5

where $D$ is the sample displacement parallel to the diffraction-plane normal, and $R$ is the radius of the diffractometer's circle. 


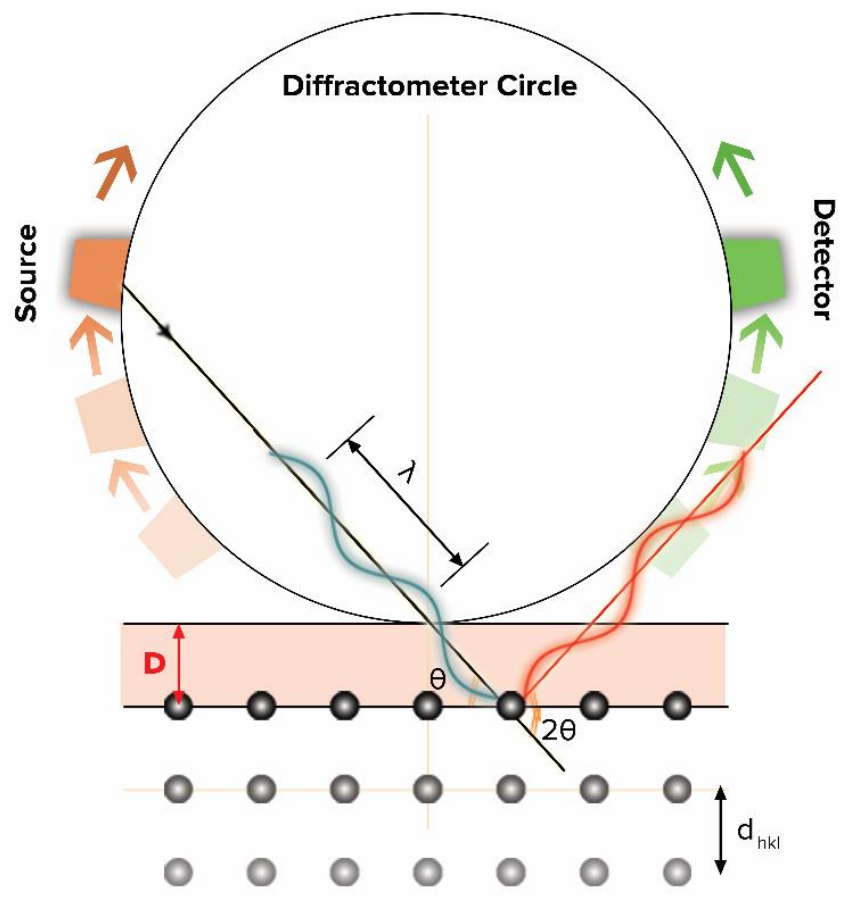

Figure 4.7: Sample displacement error gives incorrect peak positions in the resulting $\mathrm{x}$-ray diffractogram.

A relevant example for this project is evaluation of the magnitude of this error when measuring the d-spacing along the zinc oxide's (002) crystal plane orientation:

$$
\frac{\Delta d_{002}}{d_{002}}=-\frac{D \times(\cos \theta)^{2}}{R \times \sin \theta}
$$

We measured $d_{002}=2.6021917 \times 10^{-10} \mathrm{~m}, \theta_{002}=34.43508^{\circ}$; while $R$ has a value of $0.24 \mathrm{~m}$ :

$$
\Rightarrow \Delta d_{002}=-\frac{D \times(0.824767)^{2}}{0.24 \times 0.565472} \times 2.602192 \times 10^{-10}=-13.04307 \times 10^{-10} \times D
$$


Thus, if the ZnO sample was displaced by one tenth of a millimetre $(100 \mu \mathrm{m})$, the measured d-spacing along the (002) plane direction will be off by:

$$
\Delta d_{002}=-1.304307 \times 10^{-3} \stackrel{\circ}{A}
$$

\subsubsection{Stress estimation}

Lattice deformations like dislocations, grain-boundaries, and strains contribute to stresses embedded in a crystalline specimen, and have important consequences on diffraction measurements. Two types of stresses are identified in a sample, microstresses varying from one crystal grain to another, and macrostresses which extend uniformly over large distances [163]. Typical grains are slightly and randomly disoriented or rotated with respect to an ideal crystal lattice, which causes peak broadening since reflections will occur over a range of angles for a given crystal orientation. Macrostrains on the other hand, will cause variations in the lattice distances $d_{h k l}$ of a sample, which translates into peak position shifts in the corresponding diffractograms, as shown in figure 4.8. Non-uniform strains can cause a range of lattice distances to occur for a given crystal orientation, resulting in a broader and possibly non-symmetrical peak to be detected. The relation between the broadening $(\Delta 2 \theta)$ and the strain nonuniformity $\left(\Delta d_{h k l} / d_{h k l}\right)$ is given by [163]:

$$
\Delta 2 \theta=-2 \frac{\Delta d_{h k l}}{d_{h k l}} \tan \theta
$$

Equation 4.6

Thus, if extra broadening was observed -at a given diffractogram peak- above and below the instrumental width of a line, it is possible to calculate tensile and compressive strain variations along the corresponding crystallite domains. 

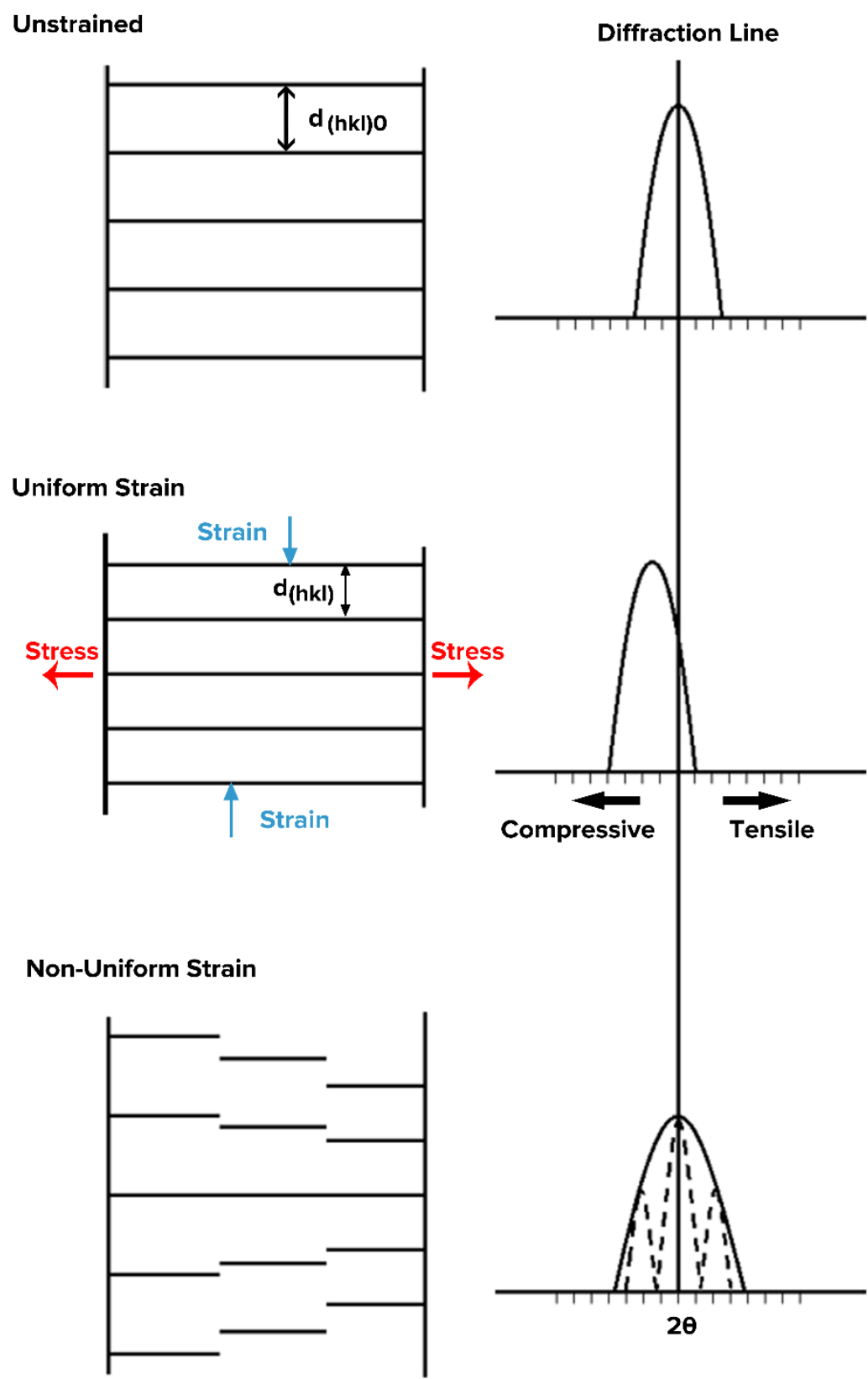

Figure 4.8: The effects of different strain types on diffraction peak position and peak width

In this project, the piezoelectric material of choice is zinc oxide, and diffractograms were used to evaluate the values of embedded stresses in the deposited films at the different processing stages. The $\mathrm{ZnO}$ crystal has a 
hexagonal lattice geometry, where the interplanar distance $d_{h k l}$ is given by [169]:

$$
\frac{1}{d_{h k l}^{2}}=\frac{4}{3}\left(\frac{h^{2}+h k+k^{2}}{a^{2}}\right)+\frac{l^{2}}{c^{2}}
$$

Equation 4.7

$h, k$, and $l$ are Miller's indices; $a$ and $c$ are the lengths of the hexagonal cell edges. Considering an unstressed $\mathrm{ZnO}$ powder sample, table 4.1 shows the available crystal orientations, with their respective lattice distances and relative peak intensities observed in a typical diffractogram [170].

Table 4.1: The different peaks expected in a standard stress-free $\mathrm{ZnO}$ power sample. Here $\mathrm{a}=3.24982 \AA$ and $\mathrm{c}=5.20661 \AA$; $\mathrm{a}$ copper anode is assumed for the $\mathrm{x}$-ray source

\begin{tabular}{llllll}
\hline \hline $\mathbf{2 \theta}\left({ }^{\circ}\right)$ & $\boldsymbol{d}_{\boldsymbol{h} \boldsymbol{k l}}(\mathbf{\AA})$ & $\boldsymbol{h}$ & $\boldsymbol{k}$ & $\boldsymbol{l}$ & Relative Intensity (\%) \\
\hline 31.802 & 2.81160 & 1 & 0 & 0 & 55.6 \\
34.447 & 2.60150 & 0 & 0 & 2 & 41.1 \\
36.290 & 2.47350 & 1 & 0 & 1 & 100 \\
47.582 & 1.90950 & 1 & 0 & 2 & 21.4 \\
56.661 & 1.62320 & 1 & 1 & 0 & 31.1 \\
62.913 & 1.47610 & 1 & 0 & 3 & 27.8 \\
66.452 & 1.40580 & 2 & 0 & 0 & 4.2 \\
68.018 & 1.37720 & 1 & 1 & 2 & 22.7 \\
69.167 & 1.35710 & 2 & 0 & 1 & 11.2 \\
72.623 & 1.30080 & 0 & 0 & 4 & 1.8 \\
77.044 & 1.23680 & 2 & 0 & 2 & 3.5 \\
81.464 & 1.18050 & 1 & 0 & 4 & 1.8 \\
89.714 & 1.09210 & 2 & 0 & 3 & 7.3
\end{tabular}


Relative peak intensities are given as percentages with respect to the highest detectable peak in a diffractogram.

The piezoelectric response in a zinc oxide crystal is aligned along the caxis, a direction which is normal to the $\{002\}$ crystal plane family; hence, we aim to produce $\mathrm{ZnO}$ films that show a high-quality dominant (002) peak in their diffractograms, with a minimal availability of any competing crystal orientations that would hinder the piezoelectric response.

Consequently, stresses in our $\mathrm{ZnO}$ films will be evaluated using the (002) peak data measured in the diffractometer. Substituting $h=k=0$ and $l=2$ in equation 4.7:

$$
\begin{gathered}
\frac{1}{d_{002}^{2}}=\frac{4}{3}\left(\frac{0+0+0}{a^{2}}\right)+\frac{4}{c^{2}}=\frac{4}{c^{2}} \\
\Leftrightarrow d_{002}=\frac{c}{2}
\end{gathered}
$$

Then, by replacing $d_{002}$ in equation 4.3 , and using the wavelength of the copper anode's characteristic line $K \alpha_{1}$, while considering the first order reflection, the relation between $d_{002}$ and $\theta_{002}$ is given by:

$$
\begin{aligned}
& 1.54056 \times 10^{-10}=2 \times d_{002} \times \sin \theta_{002}=c \times \sin \theta_{002} \\
& \Rightarrow c(\AA)=\frac{1.54056}{\sin \theta_{002}}
\end{aligned}
$$

Therefore, by measuring any deviations in the (002) peak position $\theta_{002}$, we can directly calculate the associated variation in the lattice distance $d_{002}$. The corresponding macrostrain $\varepsilon_{z}$ along the c-axis is given by: 


$$
\varepsilon_{z}=\frac{c-c_{0}}{c_{0}}
$$

Equation 4.8

where $c_{0}$ is the unstrained unit cell distance, and $c$ is the measured unit cell length. The residual in-plane stress $\sigma$ within a film can then be estimated by using Hooke's law:

$$
\sigma=\frac{1}{S} \varepsilon_{z}
$$

Equation 4.9

The constant of proportionality $S$ is the corresponding elastic compliance, which equals the inverse of stiffness. The compliance value can be estimated using the elastic constants of the material. For zinc oxide, the relevant elastic constants values are given in table 4.2 below:

Table 4.2: Elastic constants values for

a $\mathrm{ZnO}$ crystal $[154,171]$

\begin{tabular}{cc}
\hline \hline Elastic Constant & Value (GPa) \\
\hline$c_{11}$ & 208.8 \\
$c_{12}$ & 119.7 \\
$c_{13}$ & 104.2 \\
$c_{33}$ & 213.8 \\
\hline
\end{tabular}

Considering zinc oxide's hexagonal structure, the compliance's relation to the elastic constants is given by [154]: 


$$
\frac{1}{S}=\frac{2 c_{13}^{2}-c_{33}\left(c_{11}+c_{12}\right)}{2 c_{13}}=-232.812 G P a
$$

Equation 4.10

By combining equation 4.8, equation 4.9, and equation 4.10, $\mathrm{ZnO}^{\prime} \mathrm{s}$ in-plane residual stress $(\sigma)$ value is given by:

$$
\sigma(G P a)=-232.812 \times \frac{c-c_{0}}{c_{0}}
$$

Equation 4.11

\subsubsection{Error correction}

Careful visual inspection was carried out to ensure proper sample placement to minimize the dominant sample-displacement error. An estimate of the measured stress uncertainty due to the zinc oxide's sample offset by a distance of $100 \mu \mathrm{m}$ is given by:

$$
\begin{gathered}
\sigma(G P a)=-232.812 \times \frac{c-c_{0}}{c_{0}} \\
\Rightarrow \sigma(G P a)=-232.812 \times \frac{\Delta d_{002}}{d_{002_{0}}} \\
\sigma(G P a)=-232.812 \times \frac{-1.304307 \times 10^{-3}}{2.6021917} \\
\Rightarrow \sigma=0.1167 G P a
\end{gathered}
$$

Considering both scenarios where the sample could be above or below the diffractometer's circle, this stress uncertainty is given by: 


$$
\Rightarrow \Delta \sigma= \pm 0.1167 \mathrm{GPa}
$$

Thus, having even a tiny displacement error $(100 \mu \mathrm{m})$ would significantly affect the accuracy of our stress measurements, and further calibration was required.

Our solution was to make use of the sapphire substrate's (006) peak position as an anchor to a given diffractogram. Thus, a reference value for the dominant sapphire peak was identified, and any deviations from that value were assumed to be due to the wafer's displacement error. In that case, the whole diffractogram would be shifted to return the sapphire peak to its reference $2 \theta$ value.

The used sapphire substrates were cut and polished along the (006) plane orientation and were assumed to be stress-free, as provided in the supplier's specifications.

However, we were not sure if the sapphire substrates would remain stress-free after the various annealing steps, and it was important to carry out a study to determine the effects of annealing at different temperatures on the detected (006) peak position.

Several sapphire substrates were annealed at temperatures between $250^{\circ} \mathrm{C}$ and $1000{ }^{\circ} \mathrm{C}$, and several diffractograms (7 to 17) where taken for the samples at each annealing step. Then, the average and the standard-deviation for the measured sapphire's (006) peak positions were computed at each point.

The study showed that sapphire substrates remained stress-free as seen in figure 4.9, regardless of the annealing steps and temperatures. The overall average value for the measured sapphire peak position was taken as the reference anchor value: 


$$
2 \theta_{006}=41.67666^{\circ} \pm 0.02^{\circ}
$$

This $2 \theta_{006}$ value is slightly different than the $41.683^{\circ}$ reported in literature [172].

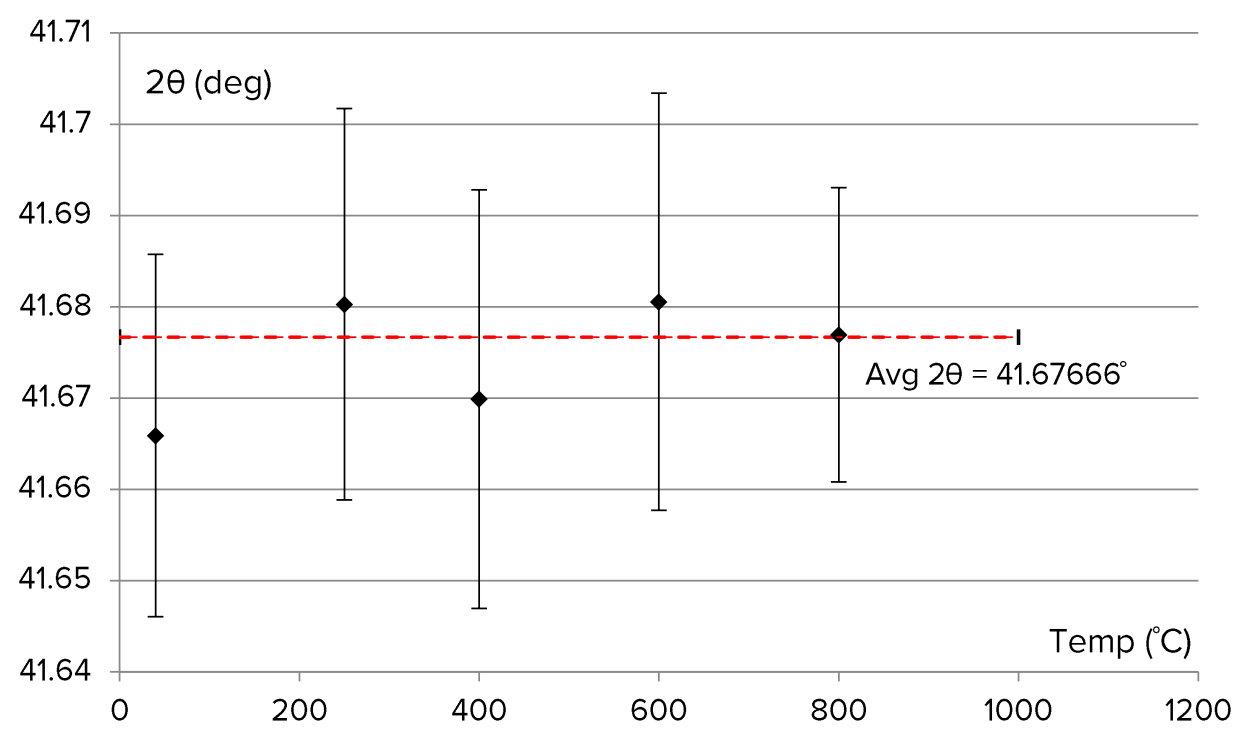

Figure 4.9: Annealing the sapphire wafers at different temperatures had no significant effect on the measured (006) peak position. The standard deviation had an approximate value of $0.02^{\circ}$

Obviously, this crucial calibration step was not applicable to samples using amorphous substrates.

\subsubsection{FWHM analysis}

Having finite crystallite domain sizes causes XRD peak broadening, as complete destructive interference will not result when Bragg's Law is not satisfied. The relation between peak broadening and the crystallite size is generally given by Scherrer's formula [173, 174, 175]: 


$$
t=\frac{K \lambda}{B \times \cos \theta}
$$

Equation 4.12

where $t$ is the mean crystallite domain size (CDS), which is less than or equal to the grain size, and is measured along a direction perpendicular to the Bragg planes; $K$ is a dimensionless shape factor with a typical value of 0.9 , but can vary from 0.62 to 2.08 [175]; $\lambda$ is the x-ray's wavelength; $\theta$ is the corresponding Bragg angle; and $B$ is the full-width at half-maximum of the peak, measured in radians.

Sherrer's formula generally applies to crystallite domains smaller than 100nm; thus, it is limited to nanoscaled crystal gain analysis [175]. During real measurements, the x-ray beam is hardly monochromatic, and non-parallel incident rays exist; these factors as well as any other imperfections will cause broader peaks to be detected.

For our $\mathrm{ZnO}$ films, it is desirable to have large crystalline domains along the c-axis, to yield a stronger and more coherent piezoelectric response across the top and bottom contacts; hence, we want our measured (002) peaks to be as narrow as possible.

\subsection{Electrical Resistance Measurements}

A two point electrical measurement was carried out to measure the DC resistance values across our zinc oxide films, as depicted in figure 4.10. The current $I(\mathrm{~A})$ was recorded while the voltage between the top and bottom contacts was swept between $-\mathrm{V}$ and $+\mathrm{V}$ and then back to $-\mathrm{V}$. The slope of the 
obtained plot equals the electrical conductance; hence, the resistance $R$ between the contacts was estimated using the slope's inverse.

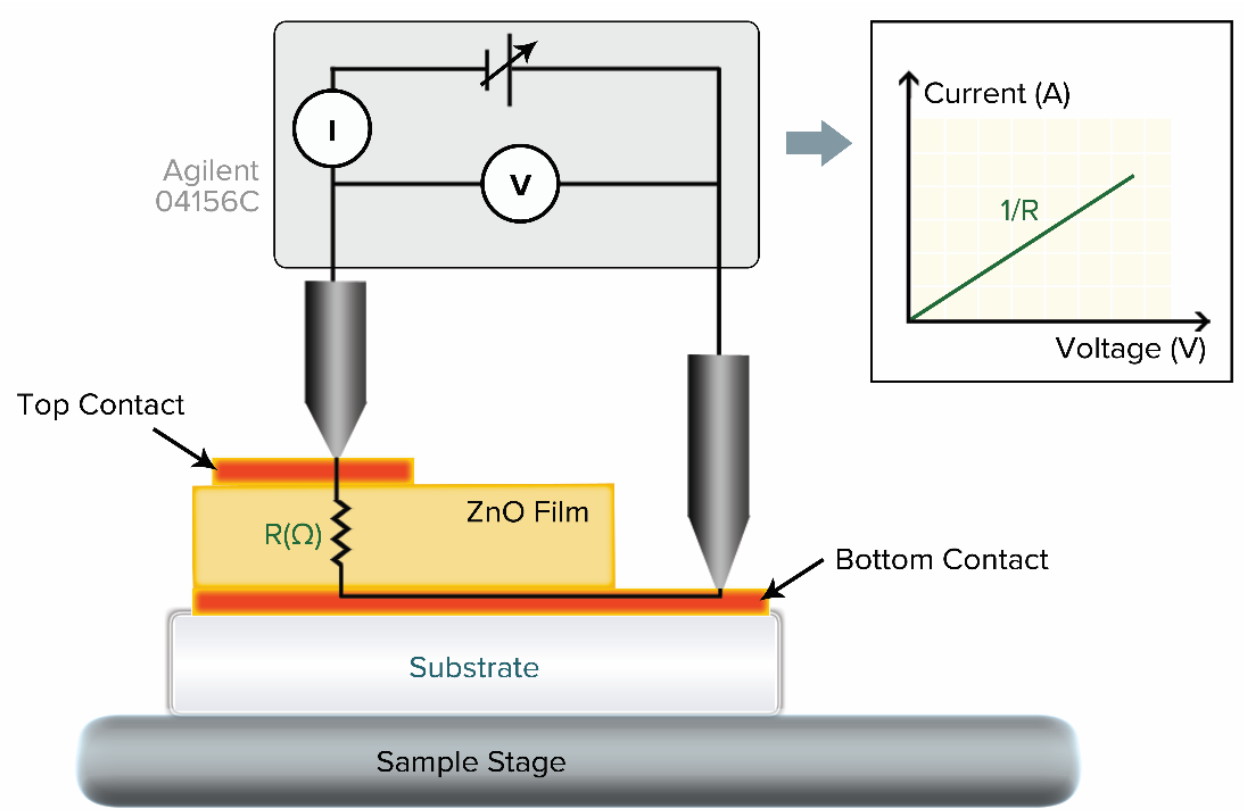

Figure 4.10: A schematic showing the setup used to measure the electrical resistance of our $\mathrm{ZnO}$ thin-films

The system used in our measurement is an Agilent 04156C, a precision semiconductor parameter analyser. It has a voltage measurement input resistance larger than $10^{15} \Omega$, and $10 \mathrm{pA}$ current resolution [176].

During measurements, the diode mode was selected. It was realized that the measured current across our thin-films was unstable in the first few seconds; therefore, at least one voltage sweeping cycle was elapsed before the current values where recorded. Regarding our settings, a medium integration time of $20 \mathrm{~ms}$ was used, and the current compliance was set to the maximum allowed value of $40 \mathrm{~mA}$. The voltage step size was set at $50 \mathrm{mV}$. 
When measuring the impedance of our samples made by the early mask designs, we had a problem due to having the top contacts on top of the $\mathrm{ZnO}$ film; thus, by slightly varying the pressure of the sensing probes, the detected current was varied by several orders of magnitude. Hence, it was very hard to get any consistent resistance values for our films. This problem was eliminated by changing the top contact structure design, which allowed probing directly on top of the substrate.

In Addition, an Agilent $4294 A$ precision impedance analyser $(40 \mathrm{~Hz}-$ $110 \mathrm{MHz}$ ) was used to measure the AC impedance magnitude and phase, across a given distance on a sample's surface.

The total of the series contact resistances was estimated by varying the distance between the probes to work out the resistivity dependency on this separation distance. The measured impedance was fitted into an $R \| C$ model, which reflected the ohmic and capacitive properties of the inspected sample.

The value of a film's sheet resistance $R_{S}(\Omega / \mathrm{sq})$ was obtained by solving the van der Pauw equation at the special case when both orthogonal resistance measurements are equal to $R$ [177]:

$$
R_{S}=\frac{\pi R}{\ln 2}
$$

Equation 4.13

Once the thickness $t$ of the measured film is known, the resistivity $\rho(\Omega \mathrm{m})$ was calculated from [178]:

$$
\rho=R_{S} \times t
$$




\subsection{SEM}

A FEI Nova NanoSEM 450 was used to obtain our high-resolution SEM images. The Nova has both a secondary electron detector for topographical contrast imaging, and a backscattered electron detector for compositional contrast imaging. Beam deceleration is optional when imaging semi or non-conductive samples. In addition, the system is equipped with an energy dispersive $x$-ray spectrometer (EDS) for elemental analysis.

A scanning electron microscope produces images by scanning a stable, well collimated, and focused electron beam along a sample's surface. These electrons interact with the sample's atoms, and various detectors are used to record the signals from these interactions [179]. Images are formed by mapping the measured signals to the lateral beam position in a raster-like fashion. Typically, the collected images contain information about a sample's surface topography and elemental composition. The three imaging modalities used in this work rely on detecting a sample's secondary electrons, backscattered electrons, and emitted characteristic x-rays.

\subsubsection{Imaging using the secondary electron detector}

Collecting low-energy $(<50 \mathrm{eV})$ secondary electrons ejected by the inelastic scattering events of the beam electrons results in images that are more sensitive to a sample's topography, as these secondary electrons originate within a few nanometers from the surface [179]. Steeper topographical variations appear brighter in this mode. 


\subsubsection{Imaging using the backscattered electron detector}

When the image is formed by detecting the highly energetic electrons that are reflected back from the sample by elastic scattering of the incident beam, compositional information regarding the sample are revealed, as the elastic back-scattering intensity depends on the atomic size and electrical properties of the interacting sample volume; heavier atoms appear brighter in this modality [179].

\subsubsection{Energy dispersive $x$-ray spectroscopy}

Other than mapped images constructed by the detected electrons resulting from the raster scanned electron beam, spectroscopic measurements can be conducted using an SEM. Energy dispersive x-ray spectroscopy relies on flooding the sample's surface with energetic electrons, having an energy larger than $12 \mathrm{KeV}$ in our study, and detecting the emitted characteristic x-rays from the sample's interaction volume. These x-rays can be used to relay information regarding the elemental composition of the sample. Point, line, and frame sampling was possible in our setup

\subsection{AFM}

A Nanosurf Naio AFM was used to provide atomic force microscopy scans at different areas of our samples. Atomic force microscopy is a type of scanning probe microscopy in which a tip is scanned along the surface of a sample while trying to maintain a constant applied force set-point. This results in a threedimensional image containing information about the sample's topography and stiffness. A typical AFM can resolve feature as small as a nanometer depending 
on the setup's isolation, insulation, and the tip's radius of curvature. The accuracy of AFM tip scanning relies on a combination of piezoelectric controllers and laser beam alignment.

Two scanning modes were used for AFM imaging in this work. In the contact mode, the tip is intended to maintain contact with the sample throughout the whole image. Therefore, this technique is prone to drifting and noise errors, where cantilevers with low stiffness are used to achieve large deflection signals while keeping the force set-point minimal. On the other hand, tapping mode was used to avoid the tip sticking to the liquid meniscus layer on a sample's surface at ambient conditions. In this mode, the cantilever is driven to oscillate near its resonance frequency, and the tip-sample force interactions impose an amplitude modulation on the resonant cantilever. This modulation can be effectively used to image a sample's surface.

To isolate random outliers and provide a better understanding of the general surface topography, values of the root mean squared roughness and profile kurtosis are provided for our AFM scans. The root mean squared roughness parameter $R_{q}$ provides an estimate for the absolute vertical deviation from the mean line measured across the surface:

$$
R_{q}=\sqrt{\frac{1}{n} \sum_{i=1}^{n} y_{i}^{2}}
$$

Equation 4.15

where the profile trace contains $\mathrm{n}$ ordered and equidistant points, and $y_{i}$ is the vertical deviation of the $i^{\text {th }}$ point from the mean. 
Kurtosis describes the distribution of the sample height data, where a surface with a few very high peaks or low valleys would give a high kurtosis, while a surface with many moderate height features gives low or negative kurtosis values. A normal distribution of features has a kurtosis of zero. The measured kurtosis parameter $R_{k u}$ is given by:

$$
R_{k u}=\frac{1}{n R_{q}{ }^{4}} \sum_{i=1}^{n} y_{i}^{4}
$$

Equation 4.16

\subsection{Ultraviolet-Visible Spectroscopy}

A Cary 100 spectrophotometer was used to measure transmission, reflection, and absorption through our samples in the ultraviolet and visible (UV/VIS) spectral regions. In this system, a deuterium lamp is used for ultraviolet illumination down to a wavelength of $200 \mathrm{~nm}$, while a tungsten halogen lamp is used to provide visible and near-infrared illumination up to a wavelength of 900nm, as shown in figure 4.11. Light is dispersed by a diffraction grating, then a narrow band is selected using a monochromator, where the band typically corresponds to $1^{\circ}$ in wavelength difference. The monochromatic beam is then split by means of a half mirror to two identical beams. Those beams traverse the same length, with one beam passing through the sample, while the other passes through a suitable reference medium. Each beam's intensity is then measured using a photo-detector, typically identical photodiodes. Thereafter, data processing is used to estimate the absorbance, reflectance, and transmittance properties of the examined sample. 
Transmission was the main UV/VIS measurement used in this work, providing a qualitative measure of sample beam intensity relative to the reference beam, as a function of the incident light frequency. Optical transmission is directly dependent on a sample's structure and composition, where photons with an energy $E=h c / \lambda$ can be absorbed to excite the sample's electrons at certain characteristic wavelengths; $h$ being Planck's constant, $c$ is the speed of light, and $\lambda$ is the incident photon's wavelength. Therefore, a transmission plot, such as that seen in figure 4.12, provides a quantitative and characteristic measure of a sample's transparency over the examined frequency range. Considering this work, we ultimately desire the whole transducer structure to be highly transparent and uniform in the visible range, to allow both optical and acoustic modalities of the SAM, as described in section 1.4.

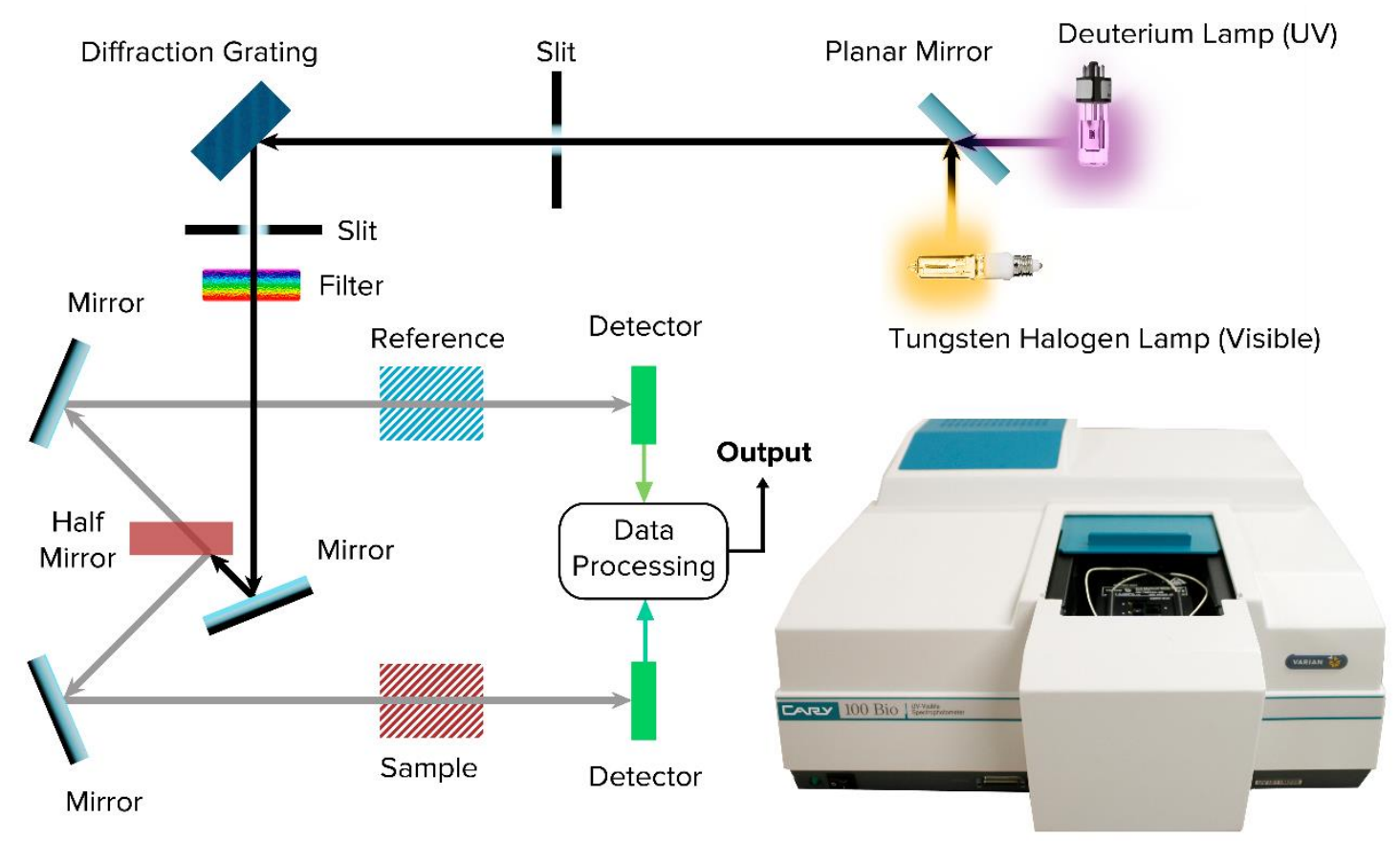

Figure 4.11: A schematic diagram showing the simplified structure of a UV/VIS spectrophotometer, with a photo of the used Cary 100 system 
Useful approximate models have been developed to estimate a sample's properties, such as the index of refraction, band gap, and thickness, using transmission measurements in low-absorbing thin-films, bounded by planeparallel surfaces, and deposited onto transparent substrates [180, 181, 182].

Thickness estimation relies on the observed fringe pattern in the highly transparent spectral region of the examined films, where fringes arise due to the multiple coherent reflections at the film interfaces. A typical example is shown in figure 4.12 .

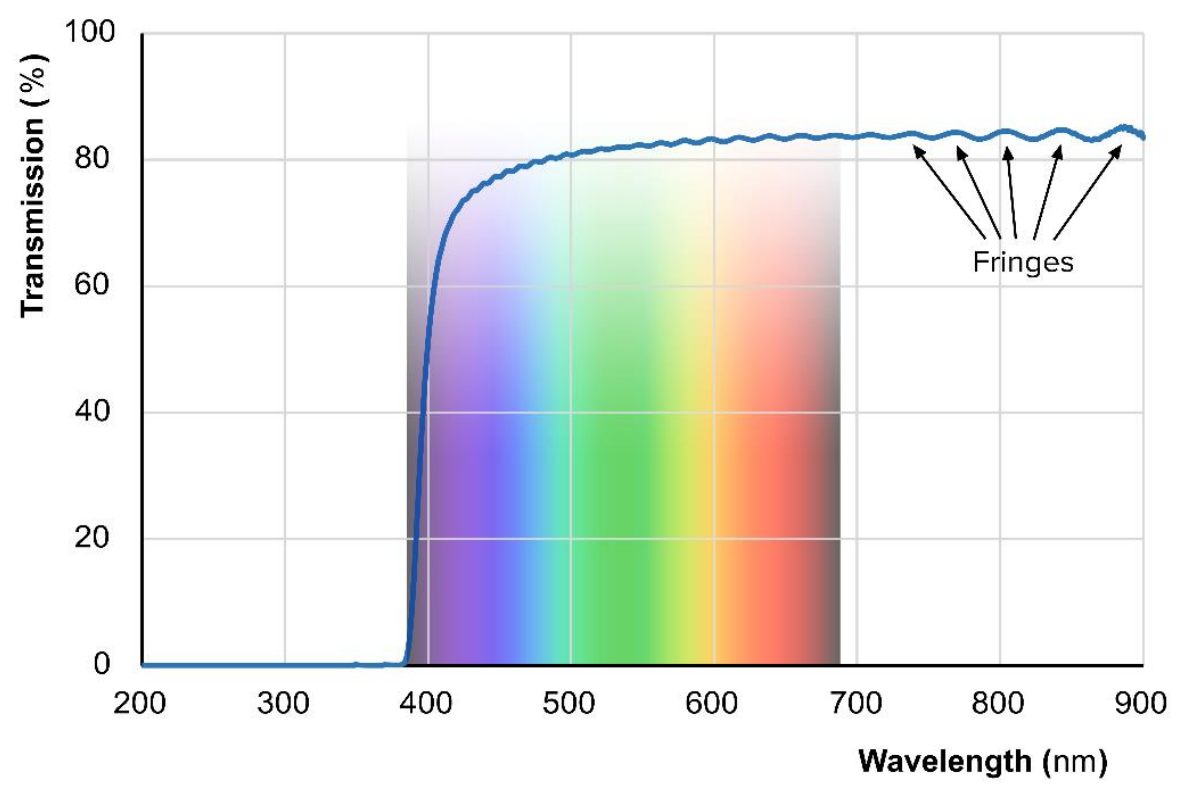

Figure 4.12: The transmittance spectrum of a $\mathrm{ZnO}$ film, with colors resembling the visible range superimposed over their corresponding wavelengths

Given the index of refraction $n$ of the film's material, the film thickness $D$ can be calculated using the following equation [180]: 


$$
D=\frac{M \times \lambda_{1} \times \lambda_{2}}{2\left[n\left(\lambda_{1}\right) \times \lambda_{2}-n\left(\lambda_{2}\right) \times \lambda_{1}\right]}
$$

Equation 4.17

where $M$ is the number of oscillations between two extrema, equal to 1 between two consecutive maxima or minima; $\lambda_{1}, \lambda_{2}, n\left(\lambda_{1}\right)$, and $n\left(\lambda_{2}\right)$ are the corresponding wavelengths and indices of refraction at the two selected extrema, respectively. To estimate the refractive index for a transparent medium at a given wavelength, the Sellmeier equation is used [183]:

$$
n^{2}(\lambda)=A+\frac{B \times \lambda^{2}}{\lambda^{2}-C^{2}}+\frac{F \times \lambda^{2}}{\lambda^{2}-G^{2}}
$$

Equation 4.18

where $A, B, C, F$, and $G$ are the Sellmeier coefficients. For $\mathrm{ZnO}$, these coefficients are $A=2.0066 ; B=1.5748 \times 10^{6} ; C=1 \times 10^{8} ; F=1.5868 ; G=2606.3$, where $\lambda$ is expressed in $\AA$ [184].

UV/VIS transmission measurements can also be used to estimate a material's optical bandgap through Tauc plots [185]. This technique uses the optical absorption spectrum to extract information regarding the electron states in the valence and conduction bands of a sample, as incident photons having energies less than the sample's band gap will not be able to excite electrons to the conduction band, thereby are transmitted. In a typical Tauc plot, $(\alpha h f)^{1 / r}$ is plotted against $h f$ near the absorption edge, where $\alpha$ is the absorption coefficient, which is a function of the wavelength, and $r$ is an exponent that accommodates the model to the correct electron transition type for a given material [186, 187]: 
- $r=1 / 2$ for direct allowed transitions

- $r=3 / 2$ for direct forbidden transitions

- $\quad r=2$ for indirect allowed transitions

- $\quad r=3$ for indirect forbidden transitions

Accordingly, the bandgap is determined by extrapolating the slope of the linear region to $\alpha \rightarrow 0$, as shown in figure 4.13. To calculate the absorption coefficient, the following approximation of the Beer-Lambert law can be used [188]:

$$
R+T=e^{-\alpha D}
$$

Equation 4.19

where $R$ and $T$ are the measured reflection and transmission magnitudes, respectively. For samples that have a high transmission and low reflection properties, $R \approx 0$ and equation 4.19 can be approximated by:

$$
\alpha=\frac{\ln \left(\frac{1}{T}\right)}{D}
$$

Equation 4.20

Using such an approximation, the absorption coefficient of the $\mathrm{ZnO}$ sample whose transmission spectrum was presented in figure 4.12 was calculated and plotted in figure 4.13. This plot was then used to estimate the value of the material's bandgap, which was found to be $3.196 \mathrm{eV}$. 
Discrepancies in this technique lie in the evident tolerance during the interpretation of the graphical representation

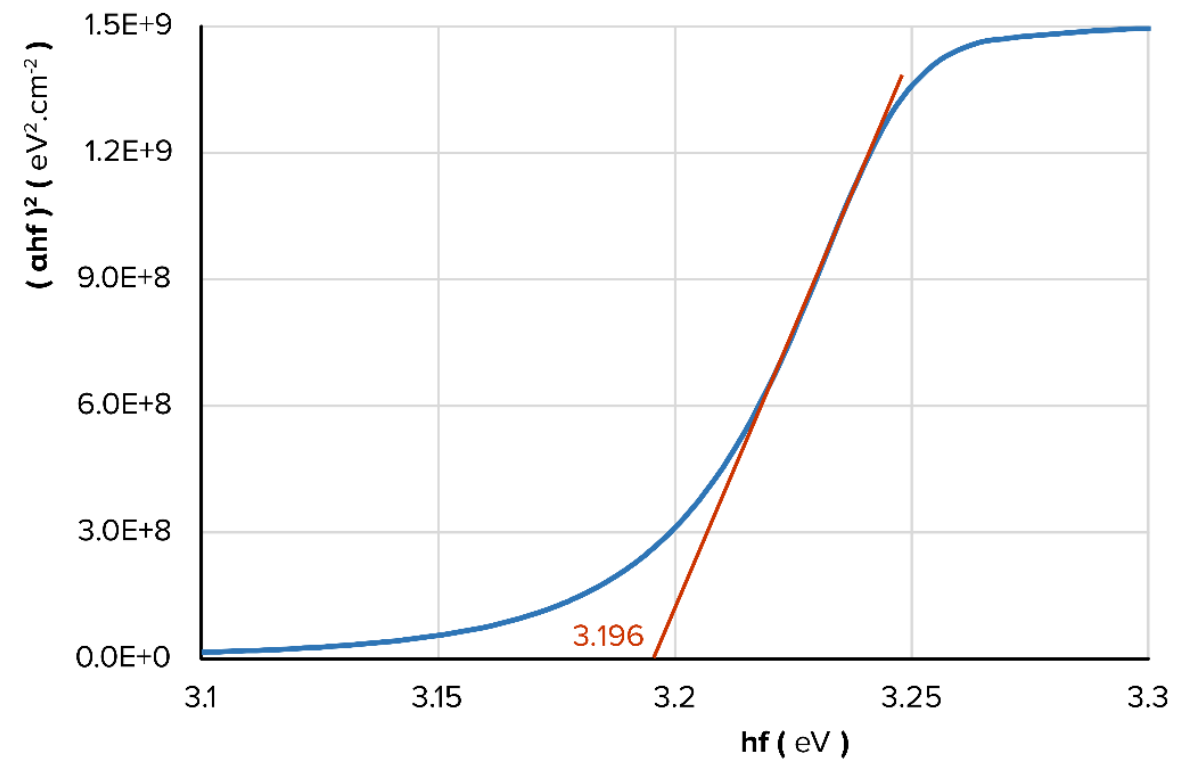

Figure 4.13: The Tauc plot for a zinc oxide sample having a thickness of $5.2 \mu \mathrm{m}$, where the extrapolated line indicates a direct bandgap of $3.196 \mathrm{eV}$. 


\section{CHAPTER 5}

\section{Results A: Sputtering parameter optimization}

The fabrication studies reported in this work aim at optimizing the structure of the sputtered zinc oxide films to suit our device application purposes. Ideally, we want our $\mathrm{ZnO}$ films to be grown in a columnar, smooth, and homogenous manner, with their c-axis aligned perpendicular to the substrate to maximize electromechanical transduction in the thickness mode [11]. Thus, the sputtered films should grow preferentially with an (002) crystal orientation, have a high electrical impedance, and the largest possible crystallite domains to maximize the piezoelectric response along the thickness direction [97, 129, 127].

\subsection{Chapter Objectives}

This chapter highlights the microfabrication steps involved in creating our targeted transducer structure. It also provides an insight to the previous relevant work done at VUW. Finally, the chapter presents the results of several 
studies that were done to optimize the zinc oxide sputtering process. The examined sputtering parameters include the structure of the bottom contact, the purity of the sputtering target, the substrate-target placement, the substrate temperature during sputtering, and the composition of the sputtering gas mixture.

\subsection{Intended Device Processing}

The main structure and steps to be applied in the fabrication process of our $\mathrm{ZnO}$ based transducer structure is shown in figure 5.1.

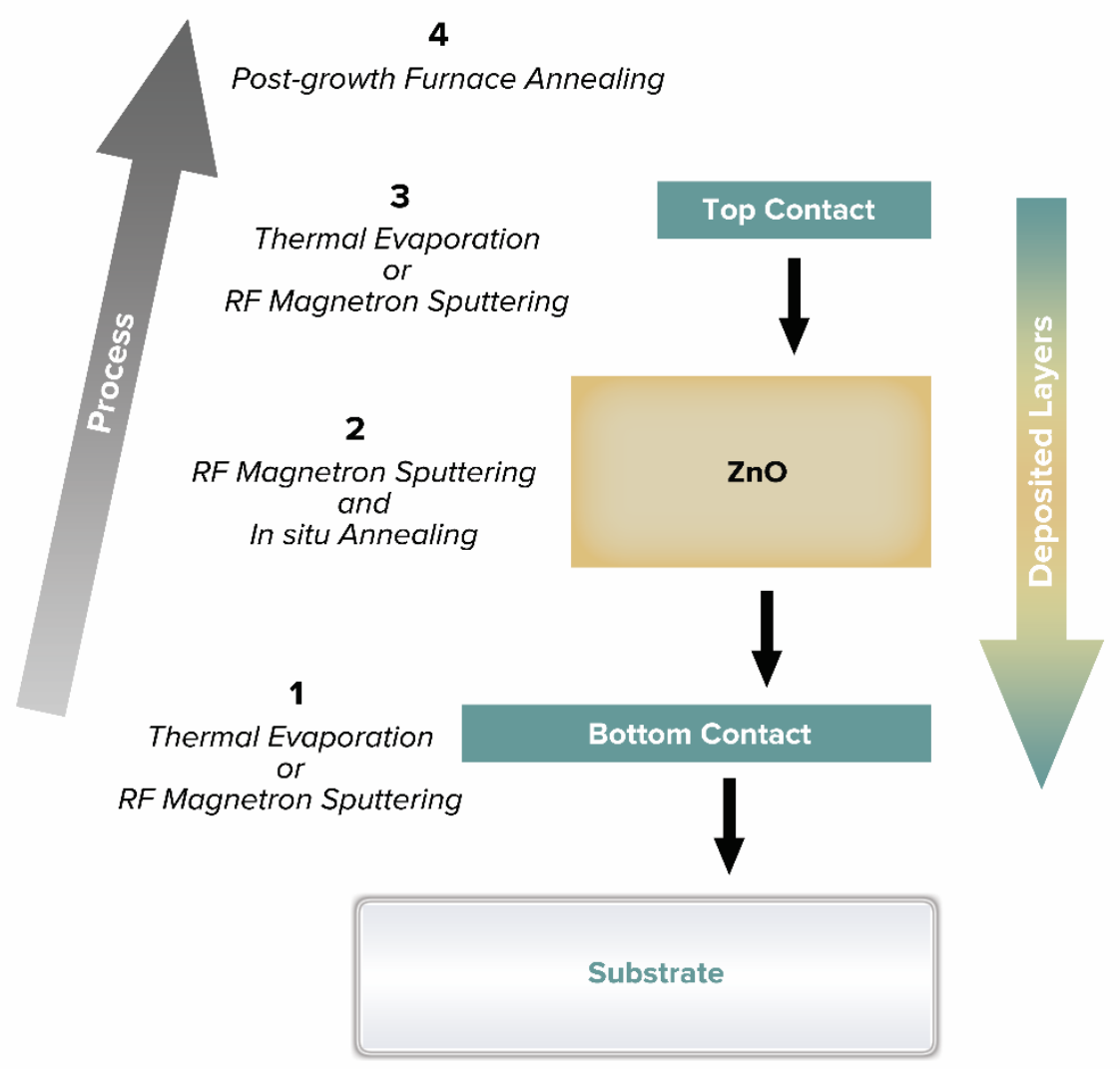

Figure 5.1: Schematic showing the different processing steps and layers of our high-frequency transducer structures 
As this project progressed, different patterning methods were used to form the intended masks for each deposited layer. This included masking by kapton tape, masking by laser-cut stainless steel sheets, and photolithography using various types of photoresists. For further details regarding the developed process, please refer to appendix B.

The general $\mathrm{ZnO}$ transducer fabrication process can be summarized in the following five steps:

1- The substrate is diced and cleaned.

2- The bottom contact mask is applied and the substrate is placed on the suitable deposition holder. Then, the bottom contact is deposited by thermal evaporation or RF magnetron sputtering.

3- The $\mathrm{ZnO}$ layer mask is applied, then the $\mathrm{ZnO}$ layer is deposited by $\mathrm{RF}$ magnetron sputtering. Thermal annealing inside the sputtering chamber can be applied during this step, a process which we refer to as in situ annealing. This annealing process does not require breaking vacuum, and is expected to relieve possible residual stress in the $\mathrm{ZnO}$ layer and to improve the film's morphology, optical, and electrical properties.

4- The top contact mask is applied, then the top contact is deposited by thermal evaporation or RF magnetron sputtering.

5- A post-growth thermal annealing process is finally carried out, to eliminate any residual stresses, grow the crystalline domain size, and improve the morphology of the deposited $\mathrm{ZnO}$ layer. This step is carried out in a tube furnace at higher temperatures and for longer times than possible in the sputtering chamber. 
Upon completing the $4^{\text {th }}$ step, a sample's thickness can be quickly measured using the profiler. A more detailed and localized topographical inspection of the sample's surface is possible using the AFM. X-ray diffraction was the primary characterization method to detect the available crystal orientations in the deposited films, and to estimate their mean crystallite domain sizes and residual stress levels. SEM imaging was also used to provide both compositional and topographical electronic images of the samples. In addition, an electrical probe station and an impedance analyser were used to measure the electrical impedance and current-voltage characteristics of the deposited films. UV/VIS spectroscopy was used to examine the reflectance, transmittance, and absorbance properties of a sample. This provided a means to estimate the bandgap and thickness of highly transparent films.

\subsection{Processing Variables}

The ability to measure the films' properties and understand how they correlate with the different processing variables was essential for establishing our optimized deposition processes. This eventually allowed the creation of homogeneous, stress-free, and c-axis oriented $\mathrm{ZnO}$ films, with a thickness exceeding $5 \mu \mathrm{m}$. Given the available resources, several processing variables were identified. These variables fall into two main categories:

a- Extrinsic variables: parameters that are external to the active layer's fabrication process. Figure 5.2 provides a summary of these variables, which can significantly affect the properties of the deposited zinc oxide films, and are related to the properties of the substrate and bottom and top contacts. Controlling such variables often requires 
choosing different materials or altering the relevant methods of preparation.

b- Intrinsic variables: these include parameters that are directly related to the zinc oxide deposition and annealing processes, and are summarized in figure 5.3. Tuning such variables can be as simple as turning a knob in our small sputtering system or complex by performing hardware modifications to adjust the system accordingly.

It should be noted that the approach relying on identifying the different processing parameters and evaluating each parameter's effects on the resulting film properties is often not sufficient, as these parameters often depend on one another. Therefore, a thorough understanding of each processing parameter's impact on the physical and chemical properties of the deposition process is rather required.

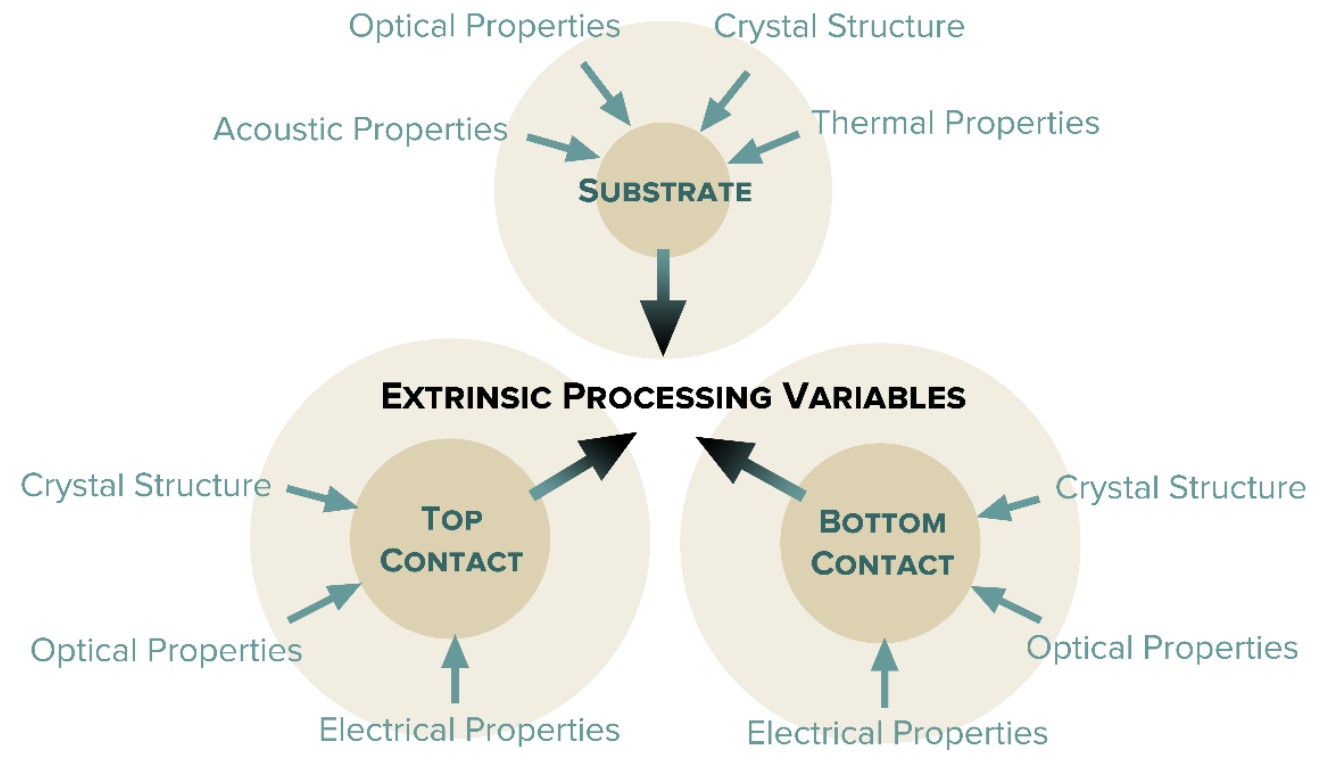

Figure 5.2: Main extrinsic variables and parameters affecting the properties of the $\mathrm{ZnO}$ transducer structures. 


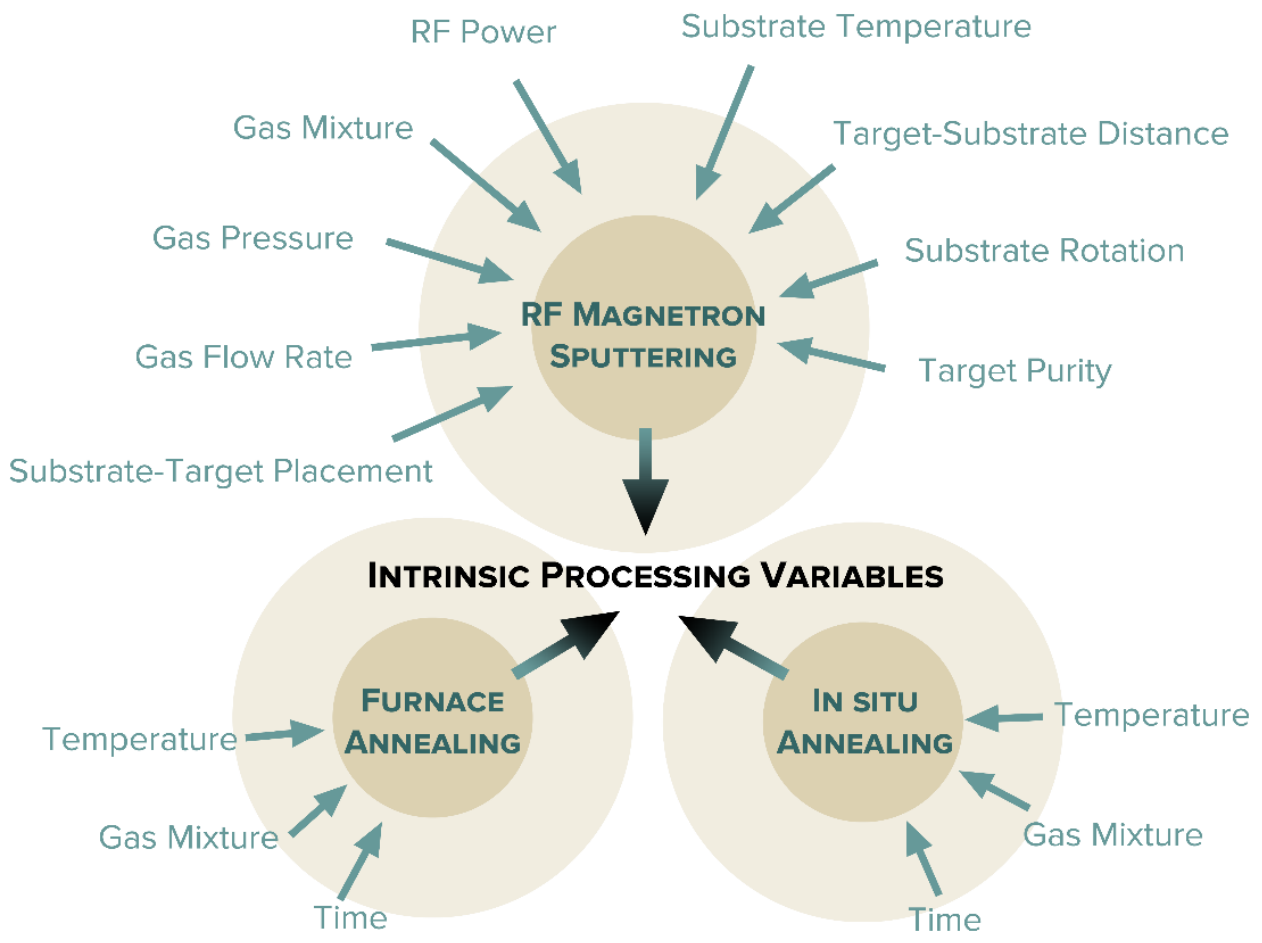

Figure 5.3: Main intrinsic variables and parameters affecting the properties of the $\mathrm{ZnO}$ transducer structures.

As RF magnetron sputtering was selected for depositing the zinc oxide films of our transducer structure, it was essential to control the sputtering plasma properties to achieve favourable film characteristics. Generally, ion bombardment within a plasma sputtering process has three direct effects on film deposition. Firstly, the temperature of the substrate rises due to the kinetic energy of the ions and the glow discharge species $[189,190]$. This will affect the surface mobility of the condensate during deposition. In addition, resputtering atoms off the deposited film is a possibility if the deposited atoms have high enough energy, or due to negative ions present within the process [191, 192]. A third effect for bombardment is the damage to the deposited film, due to the implantation of energetic atoms. Bombardment is usually tuned by means of 
adjusting the RF power, sputtering pressure, sputtering distance, substrate to target placement, and sputtering angle.

\subsection{Previous VUW Study}

Previous work [22] carried out by the same group at Victoria University of Wellington (VUW) was focused on creating c-axis oriented $\mathrm{ZnO}$ films at a moderately high deposition-rate $(1 \mu \mathrm{m} /$ hour or $16.7 \mathrm{~nm} / \mathrm{min})$, to allow the practical fabrication of thick film piezoelectric transducers. The work was done using soda-lime glass substrates, and was motivated by the acquisition of a new sputtering system by the group.

\subsubsection{Scope of the previous study}

An initial evaluation of the HHV Auto500 RF magnetron sputtering system was carried out, and several modifications were made to achieve higher deposition rates. This included placing the sample holder directly above the $\mathrm{ZnO}$ target, at a closer distance of $75 \mathrm{~mm}$-instead of $150 \mathrm{~mm}$, while not applying any substrate rotation. These steps successfully allowed the deposition of zinc oxide films at rates exceeding $34 \mathrm{~nm} / \mathrm{min}$.

Thereafter, an evaluation of the effects of the different deposition parameters on the sputtered films' quality and deposition rate was carried out. Deposition parameters included in that study were:

- $\quad$ Sputtering RF power

- Sputtering argon-flow rate

- Substrate temperature during sputtering 
- Substrate rotation

- Substrate to target placement, which accounts for the separation distance and the lateral relative positions

Most of the work done at that stage was on soda-lime glass substrates, chiefly due to their relative smoothness, acceptable thermal properties, and costeffectiveness. The results were as follows:

- It was found that c-axis oriented films could result only at moderate deposition rates, below $15 \mathrm{~nm} / \mathrm{min}$, and at a low substrate temperature, not exceeding $50^{\circ} \mathrm{C}$.

- The deposition rate was mainly controlled by adjusting the applied RF power level, where a highly c-axis oriented growth was achieved for films deposited at a low RF power. Alternatively, faster deposition rates using an RF power in excess of 240W yielded highly-stressed polycrystalline $\mathrm{ZnO}$ films with several unwanted crystal orientations. These films were useless to the intended piezoelectric application. Similar results were found by Molarius et al. [193] who recommended keeping the deposition rate below $1 \mu \mathrm{m} /$ hour to allow the fabrication of high-quality piezoelectric $\mathrm{ZnO}$ films.

- As to the effects of substrate temperature during sputtering, various literature sources $[194,195]$ argue that the condensing atoms at low or ambient temperatures will not possess enough kinetic energy to reach the position of their lowest surface energy, an aspect that is essential to form the zinc oxide's (002) crystal orientation. Therefore, substrate heating is recommended to boost such growth preferentiality. However, other studies that used similarly small sputtering systems indicated that extra crystal orientations arise while sputtering at higher temperatures 
[196, 189, 192], due to providing enough energy to enable such highenergy states. These extra orientations lead to a deterioration in the films' piezoelectric response in the thickness mode. Results at VUW agreed with the latter case, where increasing the substrate temperature during deposition did not favour the (002) orientation, but rather caused other undesired competing orientations to exist, particularly the (100) and (101) orientations. Therefore, it was recommended to avoid any substrate heating during processing.

- Increasing the substrate temperature while sputtering poses another disadvantage, due to the thermal mismatch between the substrate and the deposited layers; hence, thermal stresses were reduced when sputtering at ambient conditions. It was found that while sputtering in the absence of any heating source, stresses due to thermal expansion mismatch were negligible for the substrate materials used. This was the case for both soda-lime glass and polished borosilicate glass substrates. While sputtering at elevated substrate temperatures (up to $250^{\circ} \mathrm{C}$ ), theoretical calculations indicated that thermal expansion mismatch stresses could be neglected when compared to other sources of stress. These stresses are estimated to be of a similar magnitude even if c-axis oriented sapphire wafers are used [31, 22].

- While adjusting the sputtering gas flow-rate parameter, the highest deposition rates were achieved when having a total argon flow-rate in the 4-6sccm range.

- Maintaining an accurate and reproducible chamber pressure set-point during the deposition process was critical for establishing a good control over the residual stress level in the sputtered zinc oxide films. For a $100 \%$ 
argon gas background, pressures below $1.1 \times 10^{-2}$ mbar resulted in peeled and cracked films, whereas zinc oxide films sputtered at higher pressures were more relaxed and robust. The reduction in the residual film stress due to a higher deposition pressure has been previously reported by other groups [197, 198, 199], and could be attributed to the decreased mean-free-path of the energetic ions within the sputtering plasma, which leads to less energetic film bombardment and stress build-up. Thus, for a chosen sputtering gas flow rate, the high vacuum valve was adjusted to ensure having an optimal chamber pressure and gas flow-rate conditions during the deposition process. This allowed the creation of stress-free zinc oxide films at high deposition rates.

- Introducing oxygen to the sputtering gas mixture caused the zinc oxide films to progressively deteriorate in terms of the mean crystallite domain size and the (002) growth preferentiality. Consequently, it was decided to rely on a pure argon background during the sputtering process.

- Despite adjusting the sputtering parameters according to the aforementioned results, films thicker than $1.5 \mu \mathrm{m}$ often cracked and peeled off the substrate's surface, most likely due to stresses inherent to the sputtering process [200, 201, 202, 197]. Therefore, further process optimization was required to produce more robust $\mathrm{ZnO}$ films with thicknesses larger than $1 \mu \mathrm{m}$. Accordingly, post-growth thermal annealing in an argon-oxygen gas mixture was found useful for reducing the residual film stress level, where optimal results were achieved when annealing at a temperature of $600^{\circ} \mathrm{C}$ for 1 hour. 


\subsubsection{Recommendations of the previous study}

Based on this previous study, a four-step deposition process was suggested [22] to create thick zinc oxide films suited for transducer applications:

1- A 400-700nm thick, high-quality, and c-axis oriented $\mathrm{ZnO}$ buffer/seed layer is deposited at a low RF power of 150W. It was suggested that this buffer layer would serve as a matching substrate for further depositions. This reduces the chance of forming the unwanted orientations associated with higher deposition rates, assuming that these orientations were a direct result of the substrate or bottom contact mismatches.

2- Under the same vacuum cycle, an in situ annealing step at $250^{\circ} \mathrm{C}$ is carried out inside the sputtering chamber, to alleviate any residual stresses within the buffer layer.

3- The substrate is allowed to cool back to the ambient growth temperature, then the rest of the $\mathrm{ZnO}$ film is deposited at a higher RF power of $240 \mathrm{~W}$ to boost the overall deposition rate.

4- After the sample is removed from the sputter coater, a post-growth thermal annealing step at temperatures exceeding $400^{\circ} \mathrm{C}$ is done. This step should remove any residual stress and enhance the electromechanical and electrical properties of the films.

\subsection{Current Results}

Despite using optimal sputtering parameter settings, and following the aforementioned proposed four step deposition process [22], peeling of the deposited $\mathrm{ZnO}$ films remained a major problem especially when the sputtered 
thickness exceeded $2 \mu \mathrm{m}$. In addition, the colour and electrical resistance properties of the deposited films suffered from poor reproducibility, which was exacerbated by using targets with different purities from various vendors. Hence, the first objective of this work was to solve these problems, by isolating and studying the different variables that affect the properties of the sputtered $\mathrm{ZnO}$ layer. The considered sputtering variables included:

- Substrate material choice

- Structure of the bottom contact

- Sputtering target purity

- Substrate-target placement and the resulting film thickness uniformity

- Substrate temperature during sputtering

- Sputtering gas mixture

Throughout the following studies presented in this chapter, all three substrate materials mentioned in section 3.1.1 were used. Except for a single case, the usage of soda-lime glass substrates was discontinued in the studies presented in the next chapters. This was due to its relative low quality resulting in films peeling and having worse structural and electrical properties when compared to borosilicate glass and sapphire substrates.

\subsubsection{Structure of the bottom contact}

At the start of this project, gold was the material of choice for creating the top and bottom contact structures. As gold films tend to have poor substrate adhesion, the use of a thin chrome or titanium layer usually provides a suitable 
remedy [31, 203,24]. Due to its availability and the established processing within our lab, chrome was used for this purpose. To evaluate the importance of having a similar chromium interface layer between the gold contacts and the zinc oxide film, a test study was conducted.

Two batches of samples having the transducer structure shown in Figure 5.4 were made. For that study both borosilicate glass and sapphire substrates were used. The evaporation and sputtering conditions were kept similar, with an exception to whether the thin chromium interface layers between the gold and zinc oxide films were included or omitted. The chromium layer had a thickness of $\sim 1 \mathrm{~nm}$, while the gold contacts were $20 \mathrm{~nm}$ thick. The zinc oxide film had a total thickness of 1.4-1.6 $\mu$ m.

The resulting samples looked similar after processing was complete, with no signs of films flaking or peeling. However, samples lacking the chromium interface layers suffered from significant peeling after external thermal annealing for $1 \mathrm{hr}$ at $400^{\circ} \mathrm{C}$. The large flaking areas were observed at various places of the $\mathrm{Au} / \mathrm{ZnO} / \mathrm{Au}$ structure, irrespective of the substrate material.

As thermal annealing is an important step in the fabrication process of our final transducer structure, depositing chromium interface layers was found essential to improve the adhesion of the contacts to the substrate and to the zinc oxide film. Hence, it was decided to include the chromium layers as part of our final contact structure. 


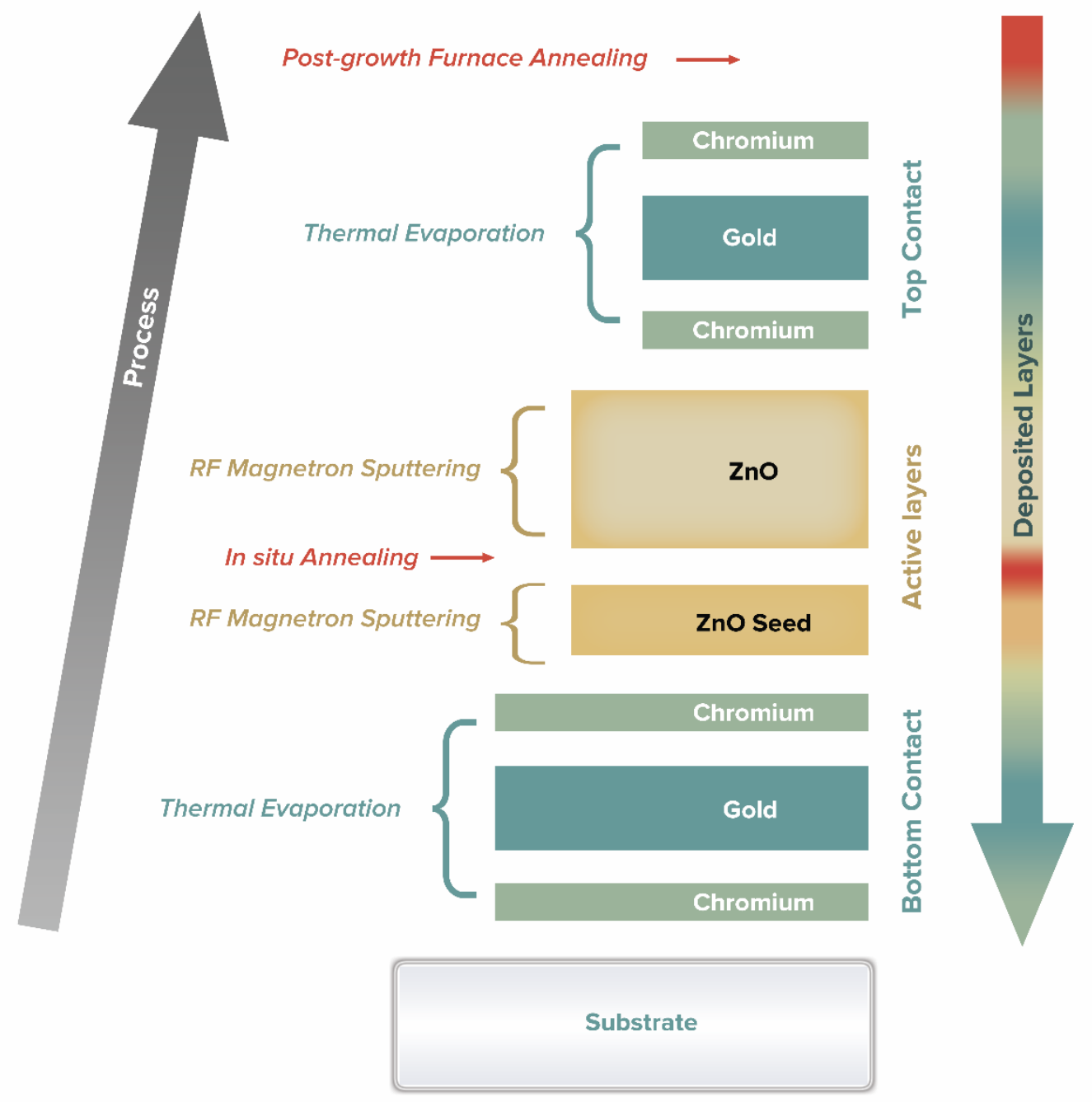

Figure 5.4: A schematic showing the deposited transducer structure

A second contact structure study was carried out to evaluate the effect of the bottom contact thickness on the quality of the sputtered zinc oxide layer. The properties of the bottom contact structure are of significant importance to this work, due to it serving as a substrate layer to the deposited $\mathrm{ZnO}$ film. In this study, both sapphire and borosilicate glass substrates were used for comparison. The deposition and sputtering parameters were kept similar, except for the bottom contact's gold layer thickness, which had three different 
values of $20 \mathrm{~nm}, 50 \mathrm{~nm}$, and $80 \mathrm{~nm}$. The deposited $\mathrm{ZnO}$ films had only the buffer layer included, which had a thickness of $\sim 0.7 \mu \mathrm{m}$. Sputtering was carried for 1hour at an RF power of $150 \mathrm{~W}$.

X-ray diffraction analysis indicated that better $\mathrm{ZnO}$ film properties; i.e., lower $\mathrm{ZnO}$ film stress and larger (002) mean crystallite domain size (t) were achieved when using thinner bottom contacts, as shown below in figure 5.5:
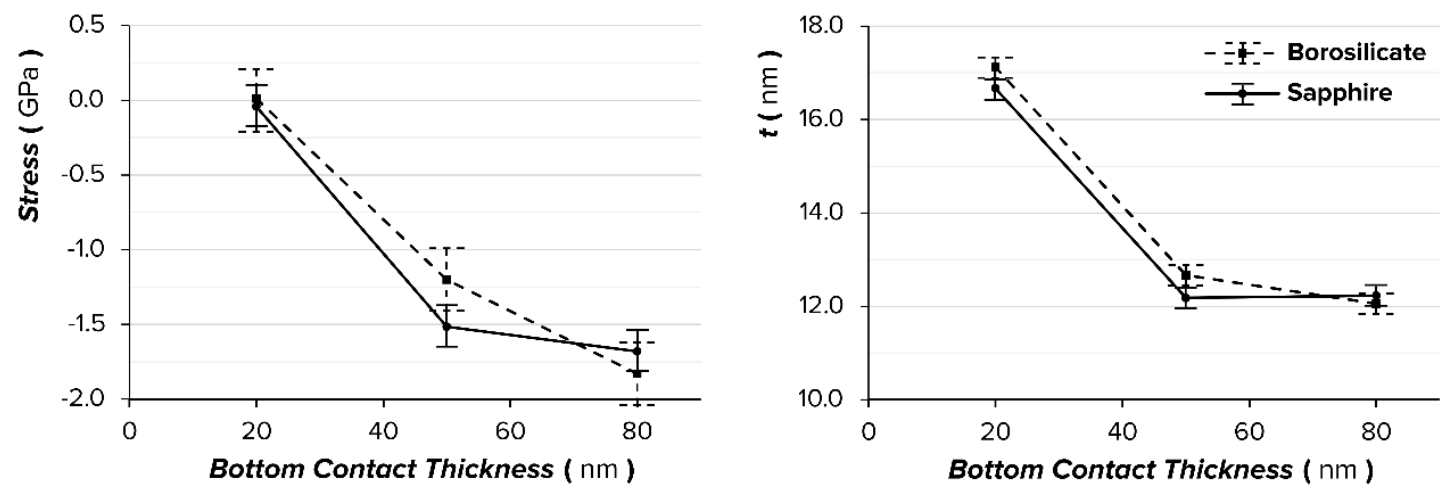

Figure 5.5: Properties of the deposited $\mathrm{ZnO}$ buffer layers on top of the 20,50 , and $80 \mathrm{~nm}$ thick bottom contacts

Evidently, it was decided to use the thinnest gold bottom contact of 20nm for later transducer structures.

\subsubsection{Target purity}

To compare the effects of target purity on the resulting $\mathrm{ZnO}$ films, buffer $\mathrm{ZnO}$ layers were deposited onto soda-lime glass, borosilicate glass, and sapphire substrates. For each sample, a similar sputtering process was conducted for 1hour at a 150W RF power, and using the same conditions, while target purity was kept as a variable. Zinc oxide targets of two different purities, 99.99\% from Ezzi Vision and 99.999\% from Kurt Lesker, were used. 
The profiler indicated that the sputtered films had comparable thicknesses of $\sim 0.7 \mu \mathrm{m}$, and x-ray diffractograms showed that film-growth was along the favourable $\mathrm{ZnO}$ (002) orientation. Expectedly, the purer target consistently yielded $\mathrm{ZnO}$ films with better diffractograms in terms of higher (002) peak intensities and smaller FWHM values. Therefore, it was decided to always use purer targets to establish better $\mathrm{ZnO}$ films.

\subsubsection{Substrate-target placement and thickness uniformity}

It is important that our high-frequency piezoelectric films have smooth morphology and uniform thickness across the deposited area, to maintain uniform electrical and electromechanical properties across the lateral dimensions of the transducer [31, 204, 9], and to allow the reproducible fabrication of complex piezoelectric transducer structures.

Therefore, a study was initiated to measure the thickness profile variation across the deposited $\mathrm{ZnO}$ films, and to evaluate the established thickness reproducibility. In this study, $\mathrm{ZnO}$ sputtering occurred on top of $\mathrm{Cr} / \mathrm{Au} / \mathrm{Cr}(2 \mathrm{~nm} / 20 \mathrm{~nm} / 2 \mathrm{~nm})$ contacts, which were deposited on soda-lime glass substrates $(7.5 \times 2.5 \times 0.1 \mathrm{~cm})$. Six different sample batches were prepared using the same sputtering conditions, where each batch consisted of two adjacent soda-lime substrates. Kapton tape was used to create a rectangular mask pattern for the deposited $\mathrm{ZnO}$ films, as seen in figure 5.6. Sputtering was done at an RF power of $150 \mathrm{~W}$ using an argon flow rate of $6 \mathrm{sccm}$ and a $99.999 \%$ pure $\mathrm{ZnO}$ target. The substrate to target distance was fixed at $66 \mathrm{~mm}$, where substrates were aligned directly above the target. No substrate heating was applied. Finally, a $\mathrm{Cr} / \mathrm{Au}(2 \mathrm{~nm} / 20 \mathrm{~nm})$ top contact structure was thermally evaporated through a metal mask. 
The thickness of the sputtered $\mathrm{ZnO}$ films was measured using the Veeco Dektak 150 surface profiler. In order to establish a thickness profile for each deposited $\mathrm{ZnO}$ batch, thickness was measured at 16 different positions, as indicated in figure 5.6. The sputtering centre was visually identified using the colourful circular pattern on each $\mathrm{ZnO}$ batch.

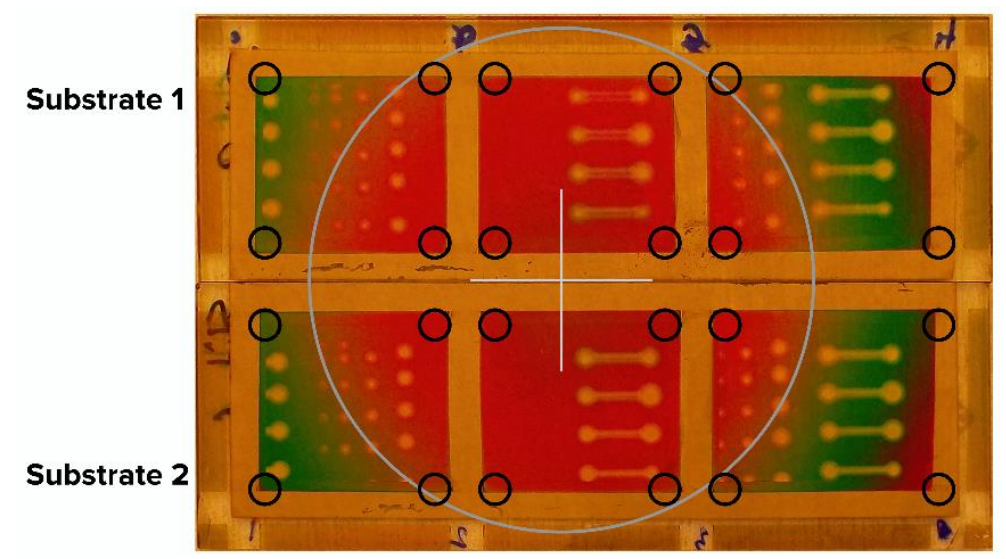

Figure 5.6: Each batch had two adjacent soda-lime substrates. For a given batch, the $16 \mathrm{ZnO}$ film edges where thickness was measured are highlighted by black circles. The central axis of deposition was visually identified using the colour pattern.

Thereafter, the measured thickness at each position was radially mapped with respect to the deposition centre, resulting in a thickness profile plot for each batch, as seen in figure 5.7. Even though the sputtering conditions were kept almost identical, thickness measurements indicated slight differences in the sputtered films thicknesses. This could be attributed to the small chamber pressure variations during deposition.

The thickness of a sputtered film radially decreases across the substrate plane, where such a gradient is expected to become more severe with a decreased target-to-substrate separation [24]. For a central deposition thickness 
of $700 \mathrm{~nm}$, the measured thickness decrease was around $150 \mathrm{~nm}$ at a radial distance of $40 \mathrm{~mm}$; this translates to a $22 \%$ decrease in the film's thickness at a radial distance of $40 \mathrm{~mm}$.

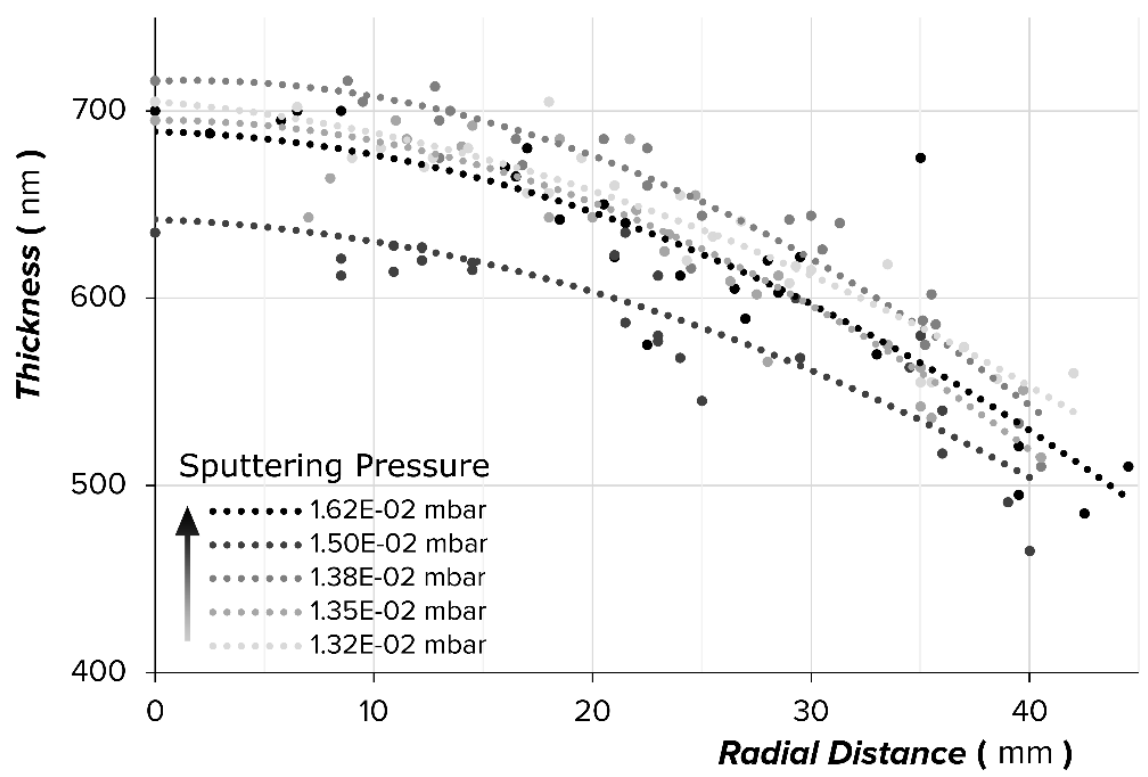

Figure 5.7: Thickness profile plots for the six different sample batches

It should be noted that the targeted single element transducer structures have much smaller lateral dimensions, of the order of a few millimetres. Hence, assuming a similar target to sample distance, the deposited thickness uniformity of our transducer structures is expected to be acceptable, with variations as little as $2 \%$ across a given element.

\subsubsection{Substrate temperature}

Using our small RF magnetron sputtering system, previous results at VUW indicated that substrate heating caused unfavourable crystal orientations to dominate during $\mathrm{ZnO}$ film deposition [22], as described in section 5.4.2. Such 
results were achieved for films deposited on soda-lime glass substrates. However, as substrate properties such as the material composition, crystal structure, and finishing have their own impact on a condensing film's energy conditions [97, 192, 24], we decided to conduct a second substrate temperature study, using borosilicate glass and sapphire substrates.

Thus, for each substrate material, a reference sample was prepared using a three-step deposition process. This process followed the recommendations and settings mentioned in section 5.4.2, while excluding the post-growth thermal annealing step. Accordingly, a thin c-axis oriented $\mathrm{ZnO}$ buffer layer was deposited at an RF power of $150 \mathrm{~W}$. Then, an in situ annealing step at $250^{\circ} \mathrm{C}$ was conducted, using a $100 \%$ oxygen gas background with a total flow rate of 6sccm. These reference samples were then allowed to cool down to a temperature of $67^{\circ} \mathrm{C}$, at which point the rest of the $\mathrm{ZnO}$ film was deposited at a higher RF power of $240 \mathrm{~W}$, for 2 hours.

An identical deposition process was carried for the rest of the samples, with an exception to the last sputtering step, which had the substrates maintained at $150^{\circ} \mathrm{C}, 200^{\circ} \mathrm{C}$, and $250^{\circ} \mathrm{C}$.

After sputtering was complete, the kapton masking tape appeared slightly burnt for samples that had substrate heating in the last deposition step. This caused the resulting $\mathrm{ZnO}$ film to appear black at several spots, as seen in figure 5.8. Several hours later, the films appeared to peel at these spots. 


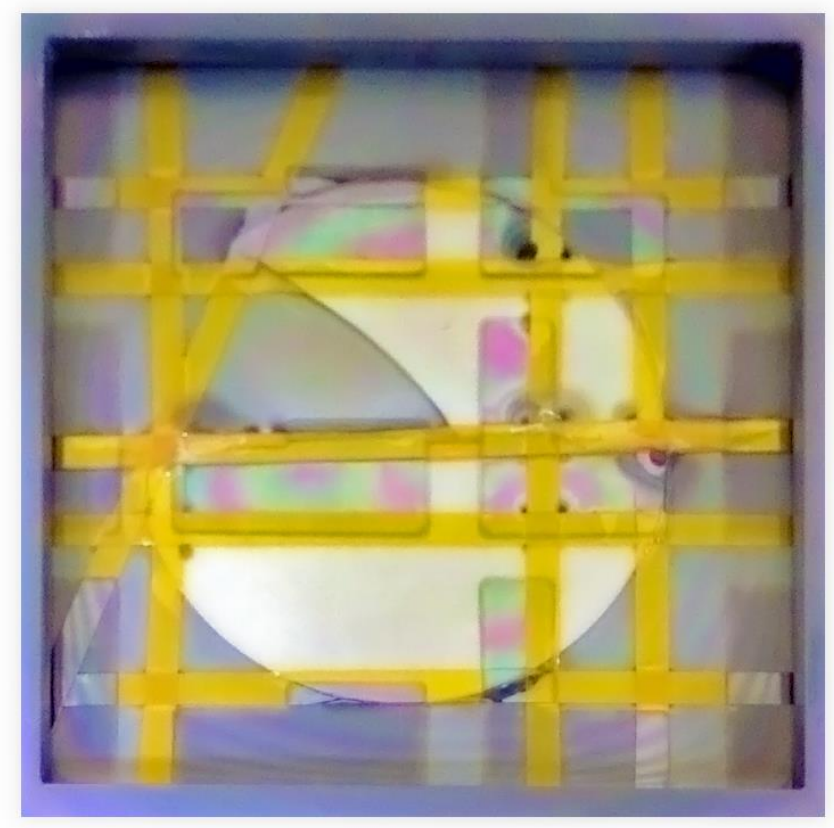

Figure 5.8: A sample after $\mathrm{ZnO}$ deposition for 2-hours at $250^{\circ} \mathrm{C}$.

The dark spots around the kapton tape edges are clearly visible.

Irrespective of the substrate material, x-ray diffractograms of the resulting $\mathrm{ZnO}$ films showed that substrate heating caused several unwanted crystal orientations to dominate, particularly the (100) and (101) orientations. The influence of substrate heating on ZnO's growth along the (002) orientation is highlighted in figure 5.9, where the $(002) /(100)$ and $(002) /(101)$ peak intensity ratios are plotted as a function of the substrate temperature.

These results are backed by the theory that thin-film surface heating due to bombardment during $\mathrm{ZnO}$ sputtering yields optimal combination of adatom mobility and kinetic energy to allow columnar c-axis smooth growth [205]. In such a scenario, having a higher substrate temperature results in excessive adatom energy that causes growth to be less susceptible to the desired shadowing effects. 
These detrimental effects of substrate heating on $\mathrm{ZnO}^{\prime} \mathrm{s}$ (002) selectivity resonate with the previous results achieved at VUW using soda-lime glass substrates [22].
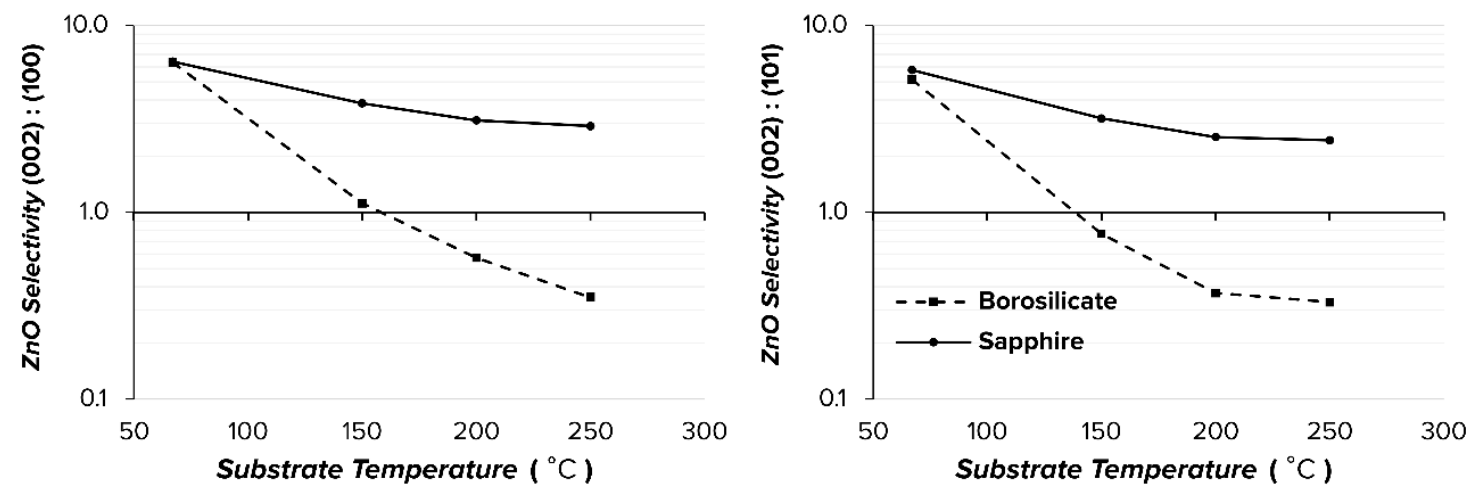

Figure 5.9: Crystal orientation selectivity of $\mathrm{ZnO}$ films deposited on both borosilicate glass and sapphire substrates as a function of the substrate temperature.

It is notable that in spite of the consistent (002) selectivity deterioration with substrate heating, sapphire substrates showed a significant (002) selectivity boost when compared to borosilicate glass substrates. Therefore, using sapphire substrates, zinc oxide growth proceeded along the favourable (002) orientation, even when the substrate temperature reached $250^{\circ} \mathrm{C}$.

As a direct result of this study, substrate heating was excluded from the $\mathrm{ZnO}$ sputtering process on top of these substrates, and kapton tape masking was avoided.

\subsubsection{Sputtering gas mixture}

Having zinc oxide films with no oxygen or zinc deficiencies improves the overall crystal symmetry, thereby, allowing a maximum piezoelectric response 
to be achieved. Moreover, deficiencies and crystal imperfections are expected to have a negative impact on the zinc oxide's semiconducting properties, due to doping effects that decrease the film's resistivity $[97,31]$. As the sputtered $\mathrm{ZnO}$ films are usually expected to be oxygen deficient [97], sputtering using an argon and oxygen gas mixture was investigated, in an attempt to optimize the resulting films' stoichiometry.

Both sapphire and borosilicate glass substrates were considered for this study. Sputtering was carried out for 1-hour at an RF power of $150 \mathrm{~W}$ using a total gas flow rate of $6 \mathrm{sccm}$. Similar deposition parameters were used to sputter the different samples, with exception to the gas mixture which considered three different constitutions:

1- $100 \%$ Ar, to provide a study reference

2- $90 \% \mathrm{Ar}: 10 \% \mathrm{O}_{2}$

3- $80 \%$ Ar : $20 \% \mathrm{O}_{2}$

The resulting $\mathrm{ZnO}$ films had a thickness of $0.6 \mu \mathrm{m}$. X-ray diffractograms indicated that having oxygen in the sputtering gas mixture resulted in films containing several undesired crystal orientations, and poor growth selectivity along the $\mathrm{ZnO}$ (002) orientation, irrespective of the substrate material used. The main competing crystal orientations were the (100) and (101), respectively, as seen in figure 5.10 .

When the oxygen content exceeded $10 \%$, the resulting $\mathrm{ZnO}$ films were no longer (002) oriented, with a slightly better (002) texture observed for films grown on sapphire substrates. 

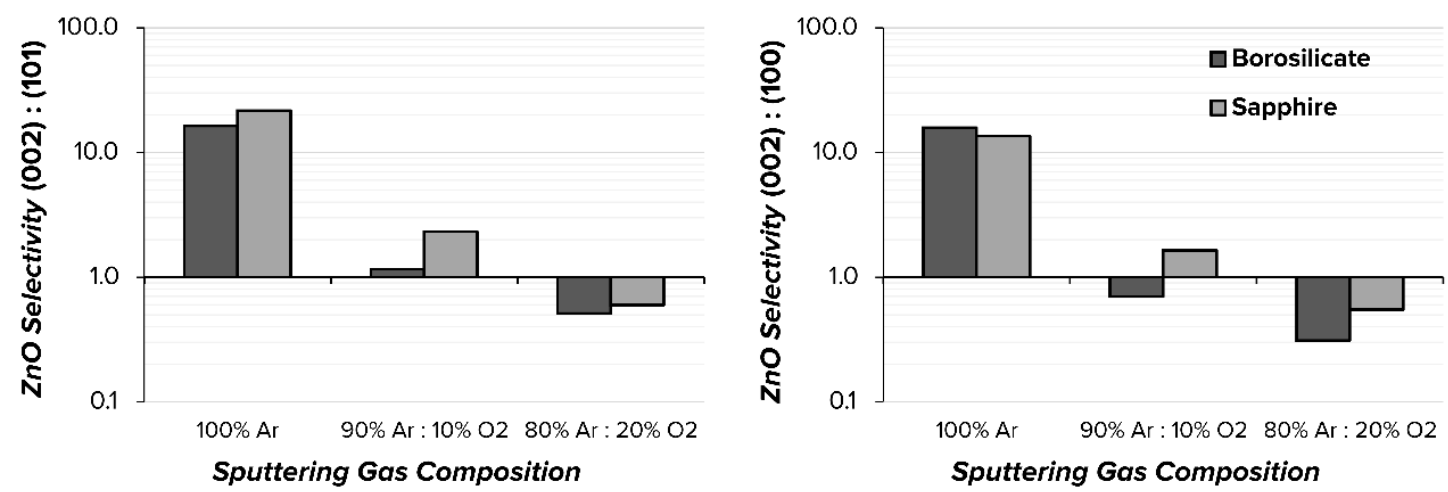

Figure 5.10: The plots show the effect of adding oxygen to the sputtering gas mixture on the resulting $\mathrm{ZnO}$ (002) selectivity.

These results are in agreement with the previous study conducted at VUW [22], where soda-lime glass substrates were used instead.

Post-growth thermal annealing of the $\mathrm{ZnO}$ films was done over 2 hours at $400^{\circ} \mathrm{C}$ and $600^{\circ} \mathrm{C}$. Annealing resulted in increased crystallite domain sizes and maximum peak intensities within the diffractograms. It also eliminated the residual film stress; however, it did not eliminate the undesirable orientations, as the (002) orientation selectivity remained constant. Hence, it was determined that a $\mathrm{ZnO}$ film should possess a high (002) orientation selectivity prior to the annealing process. It should be noted that having oxygen in the sputtering gas mixture resulted in more transparent and electrically resistive films.

As preferential growth along the c-axis orientation is essential for achieving our $\mathrm{ZnO}$ based structures, it was decided to exclude oxygen from the sputtering gas mixture in our system. 


\subsection{Chapter Summary}

This chapter provided a detailed description of the processes involved in the fabrication of our $\mathrm{ZnO}$ transducer structures. In addition, it summarized the results achieved during a previous $\mathrm{ZnO}$ sputtering study by the same research group. Finally, several sputtering studies that were carried out during the early stages of this work were presented. Following are the main findings and recommendations of the studies presented in this chapter:

- RF power exceeding 240W was found inappropriate for producing (002) oriented $\mathrm{ZnO}$ films.

- Substrate heating during sputtering resulted in having unwanted $\mathrm{ZnO}$ crystal orientations, particularly the (100) and (101) orientations. This result was also confirmed when using borosilicate and sapphire substrates.

- Pressures lower than $1.1 \times 10^{-2}$ mbar resulted in highly stressed and flaky films.

- Introducing oxygen to the sputtering gas mixture gave rise to unwanted crystal orientations. This result was confirmed for both sapphire and borosilicate substrates.

- Having a thin chromium layer was found essential for providing a better adhesion between the gold film and the substrate, as well as between the gold and the $\mathrm{ZnO}$ films.

- Having a thinner gold bottom contact layer $(20 \mathrm{~nm})$ resulted in an improved $\mathrm{ZnO}(002)$ selectivity when compared to thicker gold layers. 
- Given the relatively short target to substrate distance and the lack of rotation of the substrate holder, the thickness uniformity during deposition was found acceptable, with a thickness variation of $2 \%$ within a $1 \mathrm{~cm}$ of the film's surface.

As the different sputtering parameters were identified, and the favourable/optimal ranges and values for each of these parameters were determined, the next step was the optimisation of the $\mathrm{ZnO}$ films by means of thermal treatment. 


\section{CHAPTER 6}

\section{Results B: Thermal Annealing Effects on the ZnO Films' Structural and Electrical Properties}

After evaluating the effects of the different sputtering parameters, and optimizing these parameters to ensure creating thin zinc oxide films with properties that match our required device specifications, the next step was to examine the possibility of improving the films' properties using thermal annealing, as suggested by various sources of literature [154, 153, 206, 207, 208], and to deposit films with a thickness exceeding $2 \mu \mathrm{m}$, to test the fabrication process validity for thicker films.

\subsection{Chapter Objectives}

The main objectives of this chapter are: 
- Evaluating the effects of the in situ annealing step on the quality of the resulting $\mathrm{ZnO}$ layer.

- Evaluating the effects of the post-growth anneal step on the quality of the resulting $\mathrm{ZnO}$ layer.

- Evaluating the effects of the gas mixture composition on the results of the thermal annealing steps.

- Evaluating the effects of the substrate material choice on the results of the thermal annealing steps.

\subsection{First Assessment Test}

Soda-lime glass substrates were used to deposit the $\mathrm{ZnO}$ films in this study, where the four-step deposition process was done, according to the fabrication plan proposed in section 5.4.2, and the recommendations of the previous chapter. The deposition of the $\mathrm{ZnO}$ film at the relatively higher RF power of 240 W; i.e., the third fabrication step, was carried out for 2.5 hours. The fourth step was omitted; thus no post-growth external thermal annealing was done.

After processing was concluded, it was found that the kapton tape used to keep the wafers in place had sagged, and the resulting $\mathrm{ZnO}$ films showed visual signs of cracking and delamination, with the films resembling thin powder layers at some regions, as seen in figure 6.1.

Thickness measurements indicated that the resulting $\mathrm{ZnO}$ film had a total thickness of $3 \mu \mathrm{m}$. Peeling was especially severe at the $\mathrm{ZnO}$ film portions grown on top of the bottom metal contact. Therefore, it was crucial to solve this 
peeling problem, before any further advancement in the transducer fabrication plan could be made.
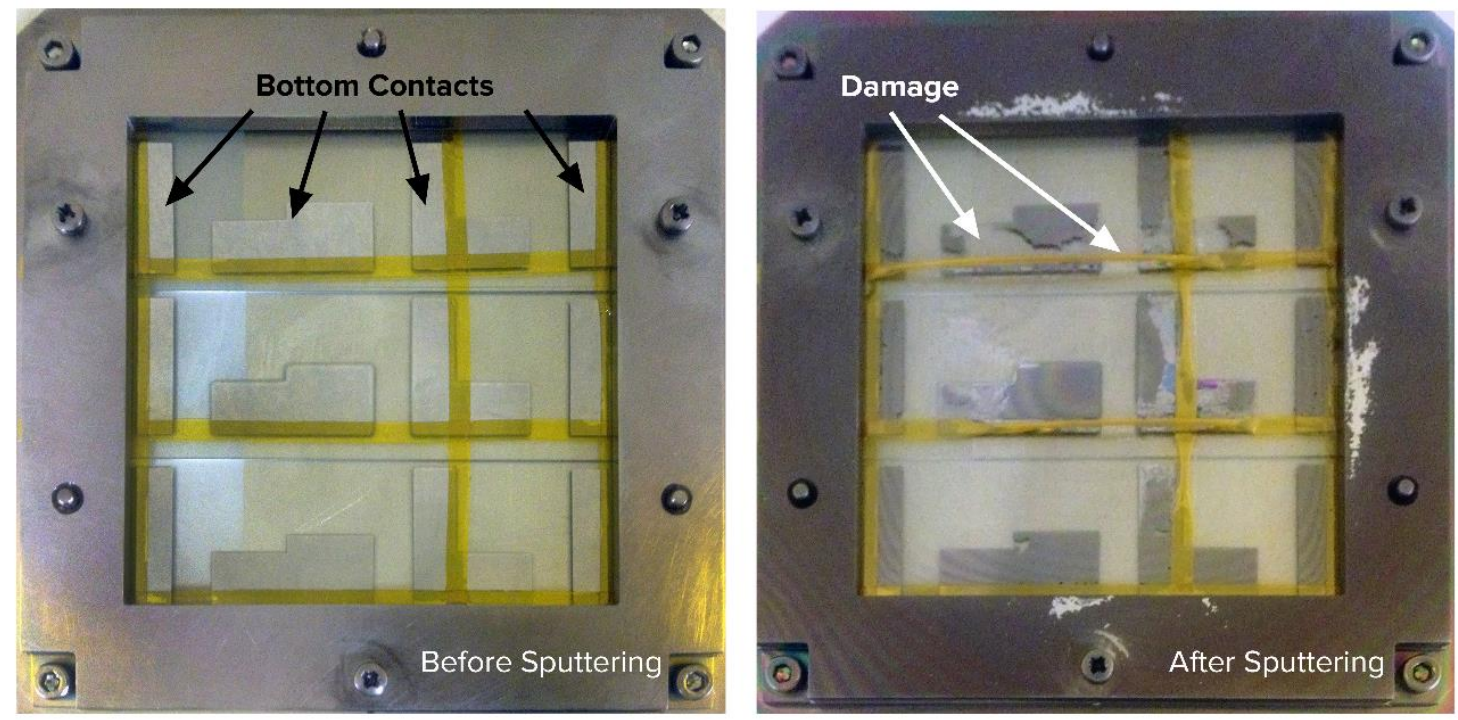

Figure 6.1: Film peeling is clearly visible on top of the metal contacts. The kapton tape sagged during sputtering, which caused non-uniform $\mathrm{ZnO}$ deposition under the sagging tape.

To eliminate the need for kapton tape during processing, a new sample holder and set of masks were made. This allowed the wafers to be kept fixed to the same holder during thermal evaporation of the metallic contacts and $\mathrm{ZnO}$ sputtering; hence, the whole process became cleaner, using robust stainless steel masks.

\subsection{In situ and Post-Growth External Annealing Study}

The in situ annealing step is carried out inside the sputtering chamber, which eliminates the need to break vacuum. The step is aimed at improving the 
structural and electrical properties of the buffer $\mathrm{ZnO}$ layer, to achieve optimized layer properties for further $\mathrm{ZnO}$ deposition at higher RF powers.

The parameters that can be controlled during this annealing step are:

- The maximum substrate temperature, which was around $250^{\circ} \mathrm{C}$, to prevent damage to the chamber components and seals.

- The time duration during which the maximum temperature is maintained. This was set to 1 hour to prevent overheating the chamber for prolonged durations.

- The gas mixture composition, with both oxygen and argon feeds being available.

- The gas flow-rate, which was kept at a total flow of $6 \mathrm{sccm}$.

- The gas pressure, which can be altered by adjusting the chamber's high vacuum valve (details in [209]). When using a total gas flow rate of $6 \mathrm{sccm}$, this chamber pressure was around $2 \times 10^{-2}$ mbar.

As the ambient gas flow-rate and pressure were held constant, and the maximum substrate temperature was used during the 1-hour in situ annealing step, the only adjustable annealing parameter was the ambient gas mixture. Adding more oxygen to the annealing gas background was expected to reduce any oxygen deficiency in the buffer $\mathrm{ZnO}$ layer. Therefore, a comparative study to evaluate the effects of the in situ annealing gas mixture on the structural and electrical properties of the deposited $\mathrm{ZnO}$ buffer layer was initiated. All three substrate materials (soda-lime glass, borosilicate glass, and sapphire) were included in this study. All these samples were later annealed in an external furnace, at temperatures higher than possible in the sputtering system, and in a 
constant 95\%:5\% Ar: $\mathrm{O}_{2}$ gas mixture. X-ray diffraction was the main tool used for characterizing the structural properties of the resulting films (details in section 4.2), whereas an electrical probe station (details in section 4.3) was used to measure the electrical resistance of the films.

\subsubsection{Soda-lime glass}

Sputtering occurred on top of $\mathrm{Cr} / \mathrm{Au} / \mathrm{Cr}(2 \mathrm{~nm} / 50 \mathrm{~nm} / 2 \mathrm{~nm})$ contacts, which were deposited on soda-lime glass substrates (measuring $7.5 \times 2.5 \times 0.1 \mathrm{~cm}^{3}$ ). Five different samples were prepared using the same sputtering conditions. Thus, the RF power was set at $150 \mathrm{~W}$, the argon flow rate was maintained at $6 \mathrm{sccm}$, with a 66mm substrate to target distance, and using a $99.999 \%$ pure $\mathrm{ZnO}$ target. The substrate was not heated to avoid the introduction of unwanted crystal orientations. The only difference between the five samples concerned the composition of the gas mixture during the in situ annealing step, which lasted for one hour at a temperature of $250^{\circ} \mathrm{C}$ :

- Sample-1: total flow 6sccm, 100\% Ar

- Sample-2: total flow 6sccm, 75\% Ar, $25 \% \mathrm{O}_{2}$

- Sample-3: total flow 6sccm, 50\% Ar, 50\% $\mathrm{O}_{2}$

- Sample-4: total flow 6sccm, 25\% Ar, 75\% $\mathrm{O}_{2}$

- Sample-5: no in situ annealing (to act as a reference)

A top contact layer was then deposited to allow the electrical characterization of the $\mathrm{ZnO}$ layers. Profiler measurements indicated that the $\mathrm{ZnO}$ films had a thickness of 500-700nm, which is consistent with the expected deposition rate. 


\subsubsection{Structural properties}

$\mathrm{X}$-ray diffractograms of the different $\mathrm{ZnO}$ samples indicated that the sputtered films had a favourable growth along the (002) orientation, as no other orientations were detected. As seen in figure 6.2, the films possessed residual compressive stresses, where these stresses were largest when no in situ thermal annealing was applied; i.e., in the case of sample-5. As the $\mathrm{ZnO}$ films were deposited on soda-lime glass, a major source of error in residual stress estimation using x-ray diffractograms arises due to the inability to measure an absolute $2 \theta$ value for a given peak, by utilizing well-defined peak positions, such as the (006) sapphire peak (details in section 4.2). The full-width at halfmaximum (FWHM) values were comparable for all the samples, indicating that the crystallite-domain sizes were not affected by the variations in the in situ annealing step.
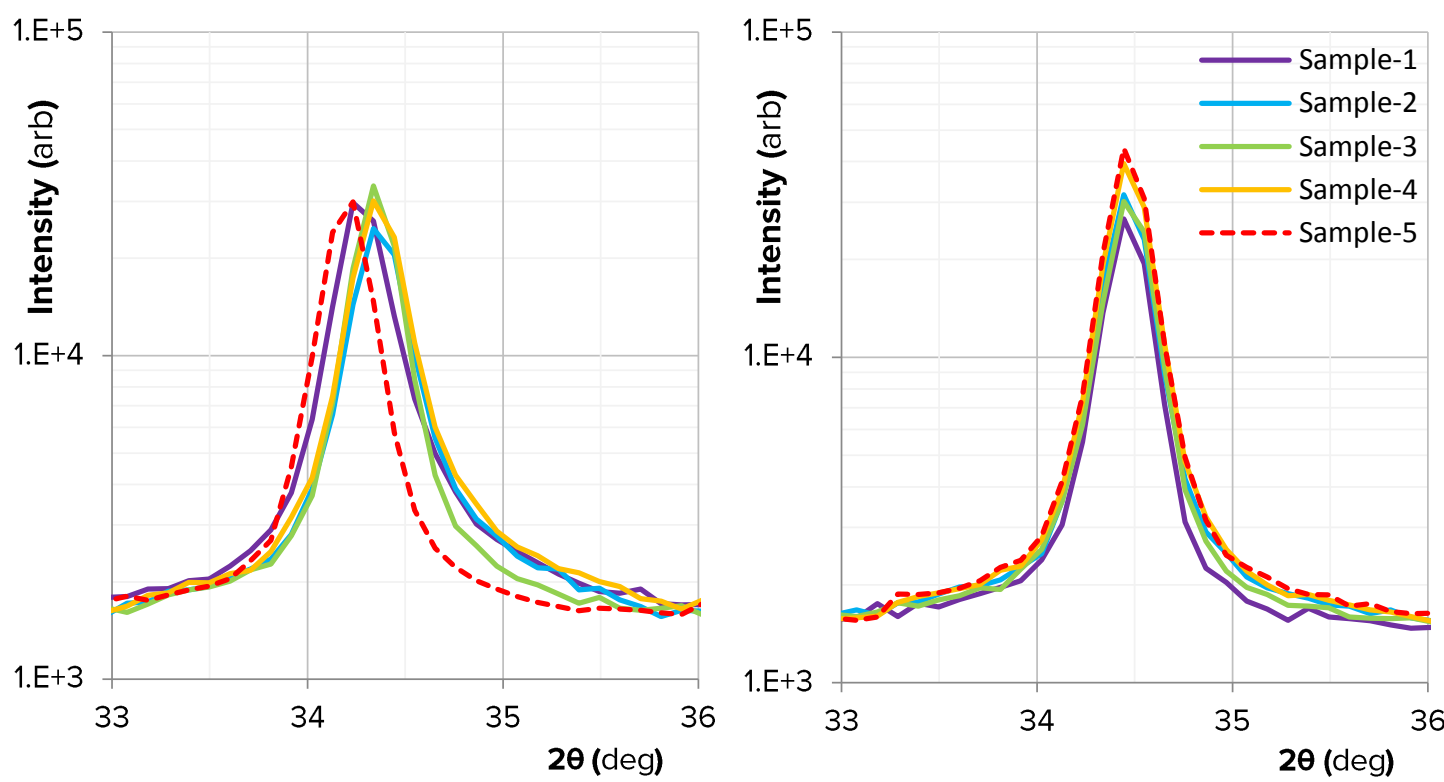

Figure 6.2: X-ray diffractograms of the different $\mathrm{ZnO}$ samples deposited onto soda-lime substrates, showing the $\mathrm{ZnO}$ (002) peak. Left) After the in situ annealing step; Right) After the external annealing step. 
The samples were later annealed in an external furnace, as described in section 3.3 , for one hour at a temperature of $400^{\circ} \mathrm{C}$, and in a $95 \%: 5 \% \mathrm{Ar}: \mathrm{O}_{2}$ gas mixture. After this external annealing step, the estimated $\mathrm{ZnO}(002)$ peak positions for the different samples became well aligned, suggesting that the residual stress became slightly tensile and almost identical in all samples, as seen in figure 6.2 and figure 6.3.

In addition, the estimated FWHM values decreased, with increased maximum count rates of the (002) peaks. Having smaller FWHM values indicated that that average crystallite domain size became larger due to annealing at $400^{\circ} \mathrm{C}$.

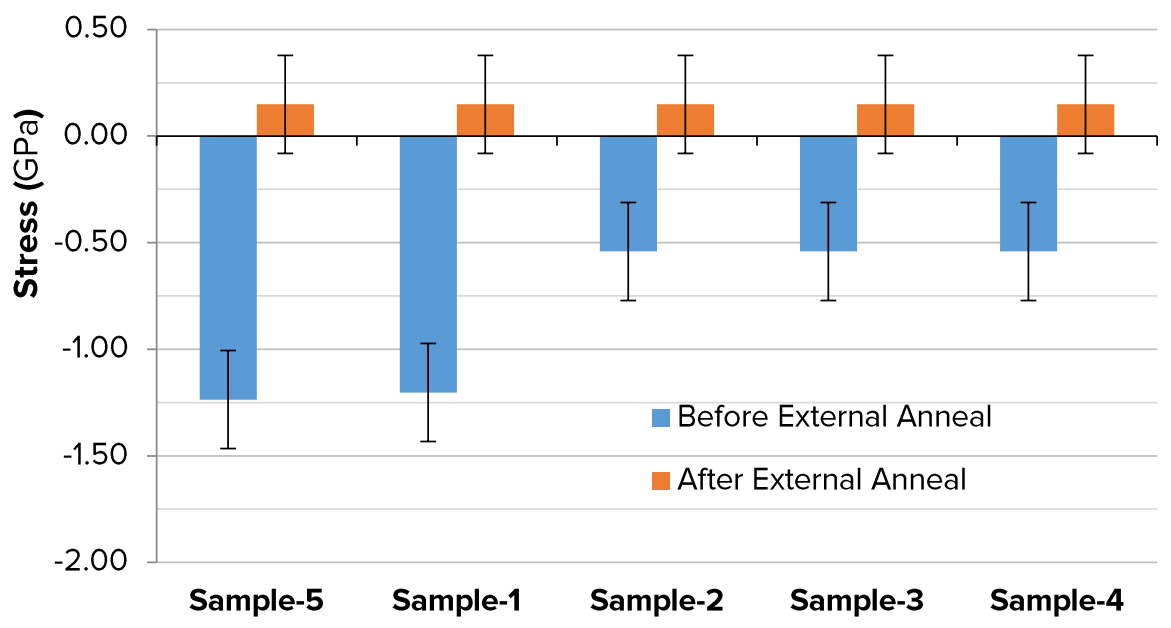

Figure 6.3: The estimated residual stress levels for the different $\mathrm{ZnO}$ samples. Negative stress values indicate compressive stress, whereas positive values indicate tensile stress.

Therefore, this study emphasized the need to carry out the in situ annealing step, as it helped reduce the residual stress level in a sputtered $\mathrm{ZnO}$ buffer layer. The external anneal step at $400^{\circ} \mathrm{C}$ was found enough to eliminate the 
inherent compressive film stress, and resulted in a slightly larger crystallite domain size.

\subsubsection{Electrical properties}

Having highly resistive piezoelectric films with small resistance variation across the films' surface area is an essential requirement for this project. Thus, a parameter analyser (details in section 4.3) was used to measure the electrical resistivity across the different $\mathrm{ZnO}$ films of this study.

Despite being only 700nm thick, electrical resistance measurements showed that the buffer $\mathrm{ZnO}$ films had a resistivity of at least $50 \Omega . \mathrm{cm}$, and up to $1 \times 10^{7} \Omega . c m$, which was actually limited by the analyser's internal resistance. Figure 6.4 shows the measured electrical resistivity versus the radial distance from the sputtering centre for all samples.

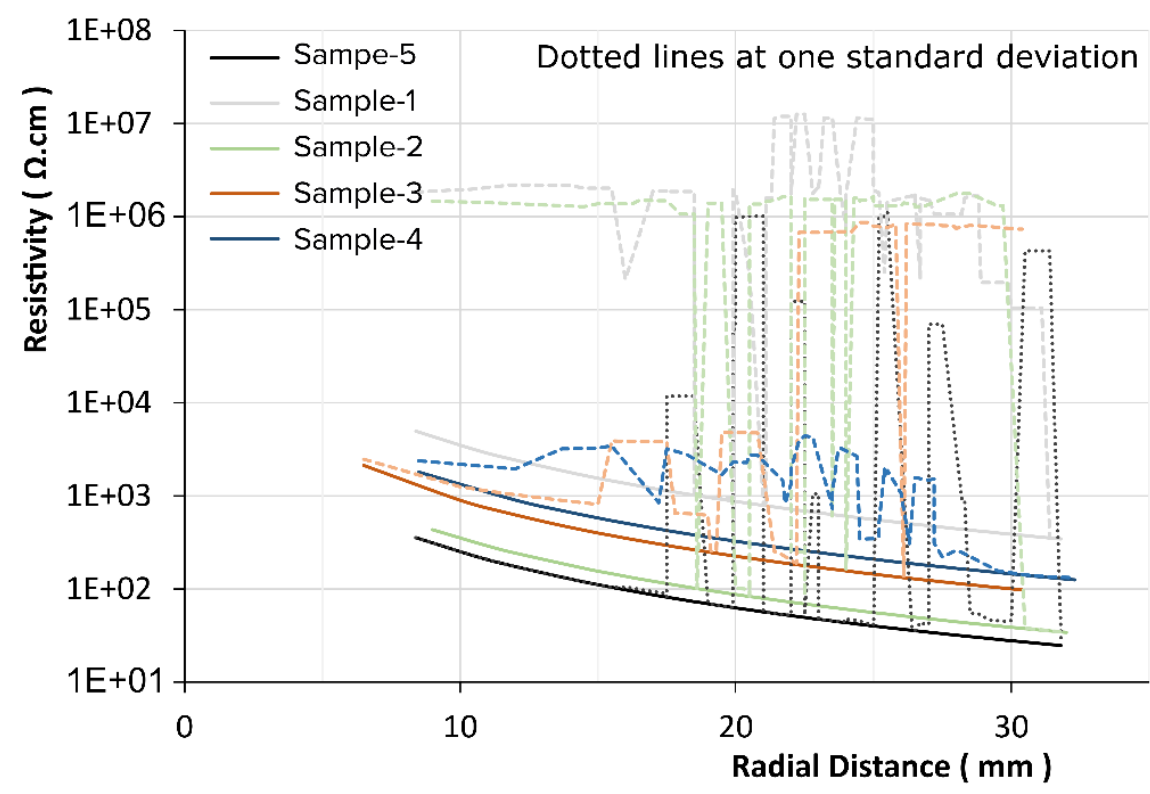

Figure 6.4: The average measured electrical resistivity values versus the radial distance from the sputtering centre for the 5 samples of this study. For each sample, the dotted line is located at a single standard deviation from the mean. 
A more interesting finding was the dependence of the films' electrical resistance on the composition of the gas mixture during the in situ annealing step. Thus, the results indicated that the in situ annealing step was necessary to ensure having a reproducible resistive buffer $\mathrm{ZnO}$ layer. Using more oxygen in the in situ annealing gas-mixture resulted in films having more stable electrical resistivity values across the different areas of the film.

Samples lacking the in situ annealing step or having an oxygen flow lower than $50 \%$ of the total annealing gas-mixture had large variations in the measured resistivity values, sometimes spanning up to 7 orders of magnitude, which imply that such films were not guaranteed to have reproducible resistive properties. Therefore, the in situ annealing step was found necessary to optimize the electrical properties of the buffer $\mathrm{ZnO}$ layer, as having more oxygen in the annealing gas-mixture resulted in stable resistive films.

\subsubsection{In situ anneal of borosilicate and sapphire samples}

Given the same exact settings used for depositing the buffer $\mathrm{ZnO}$ films on top of soda-lime glass substrates (section 6.3.1), two more sample batches were prepared using borosilicate glass and sapphire substrates. Similarly, in situ annealing for one hour at $250^{\circ} \mathrm{C}$ and using different gas mixture compositions resulted in five different samples for each substrate material:

- Sample-1: annealing gas mixture was 100\% Ar

- Sample-2: annealing gas mixture was 70\% $\mathrm{Ar}, 30 \% \mathrm{O}_{2}$

- Sample-3: annealing gas mixture was 30\% $\mathrm{Ar}, 70 \% \mathrm{O}_{2}$ 
- Sample-4: annealing gas mixture was $100 \% \mathrm{O}_{2}$

- Sample-5: no in situ annealing step, to act as a reference

Profiler measurements indicated that the $\mathrm{ZnO}$ films had a total thickness ranging from $450 \mathrm{~nm}$ to $600 \mathrm{~nm}$.

\subsubsection{Structural}

\subsection{Borosilicate glass}

X-ray diffractograms of the different $\mathrm{ZnO}$ samples indicated that the sputtered buffer layers had a poor (002) selectivity, except for the case when only oxygen gas was used during the in situ annealing step (sample-4), as seen in figure 6.5.

Similar to the soda-lime glass samples, it was not possible to adjust the diffractograms of these films to eliminate misalignment errors, due to the amorphous nature of the substrate, and the lack of any well-defined peak (details in section 4.2). Nonetheless, it was found that the in situ annealing step contributed to having films with reduced residual stress levels, where the unannealed reference sample had the highest stress level, while the in situ annealed samples had slight tensile stresses.

Moreover, as in the case of soda-lime glass substrates, the full-width at half-maximum (FWHM) values were comparable for all the samples, indicating that the crystallite-domain sizes were not affected by the in situ annealing step, as seen in figure 6.5 . 

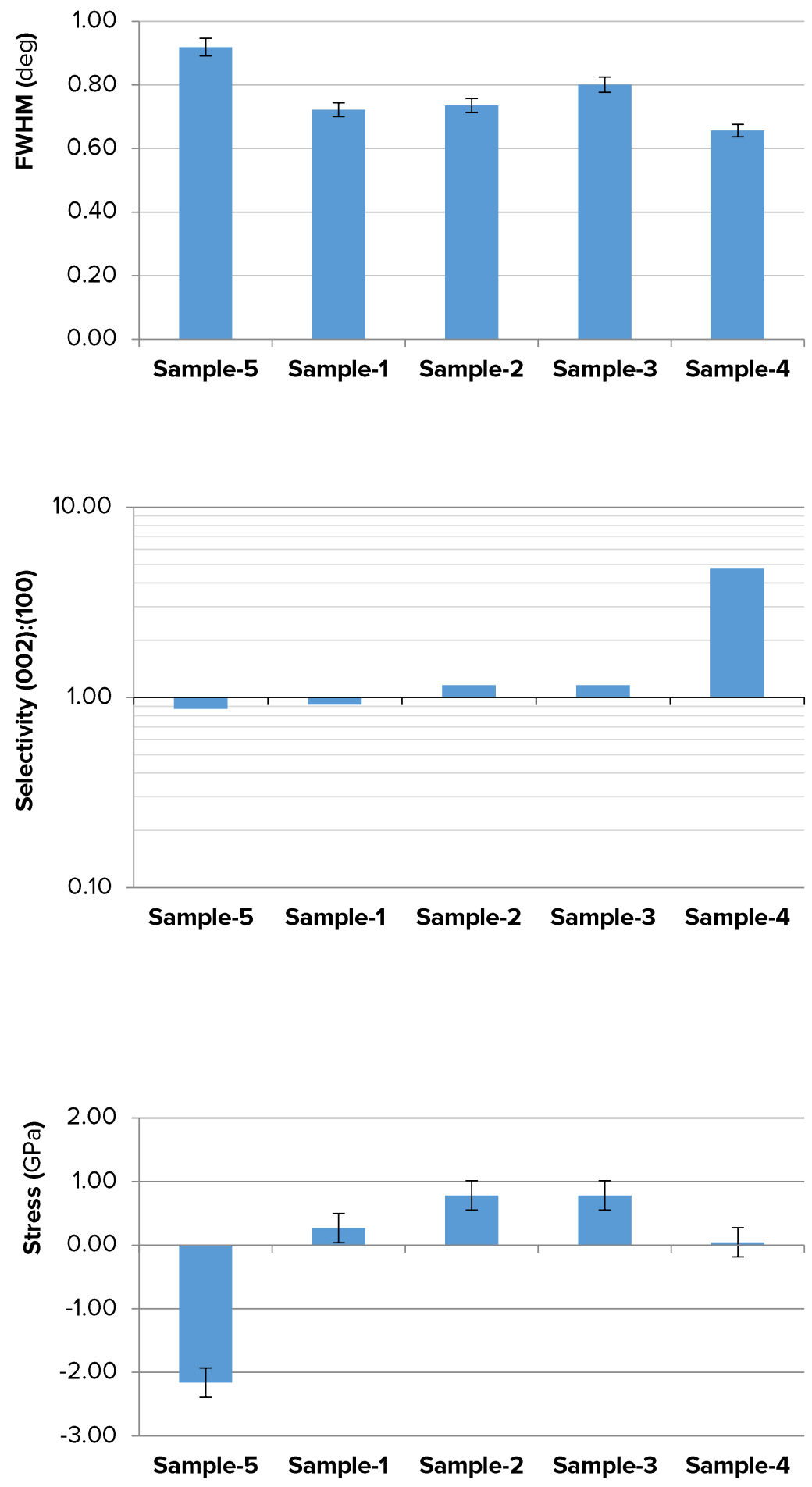

Figure 6.5: Plots showing the estimated structural properties for the 5 different $\mathrm{ZnO}$ samples on top of borosilicate glass; top) FWHM, middle) ZnO (002):(100) selectivity , bottom) Residual stress level 
During sample fabrication in this study, certain regions were intentionally left out without a bottom contact structure, to allow direct $\mathrm{ZnO}$ deposition onto the borosilicate substrate. This gave us the ability to understand the effects of the bottom contact layer on $\mathrm{ZnO}$ growth. The most significant finding from the $\mathrm{x}$ ray diffractograms measured for films directly grown on the substrate was related to the (002) crystal orientation selectivity; the result indicated a significant boost in (002) growth selectivity when $\mathrm{ZnO}$ was sputtered directly on the substrate (Figure 6.6). Therefore, the bottom metal contact had a significant detrimental effect on the $\mathrm{ZnO}$ (002) orientation selectivity.

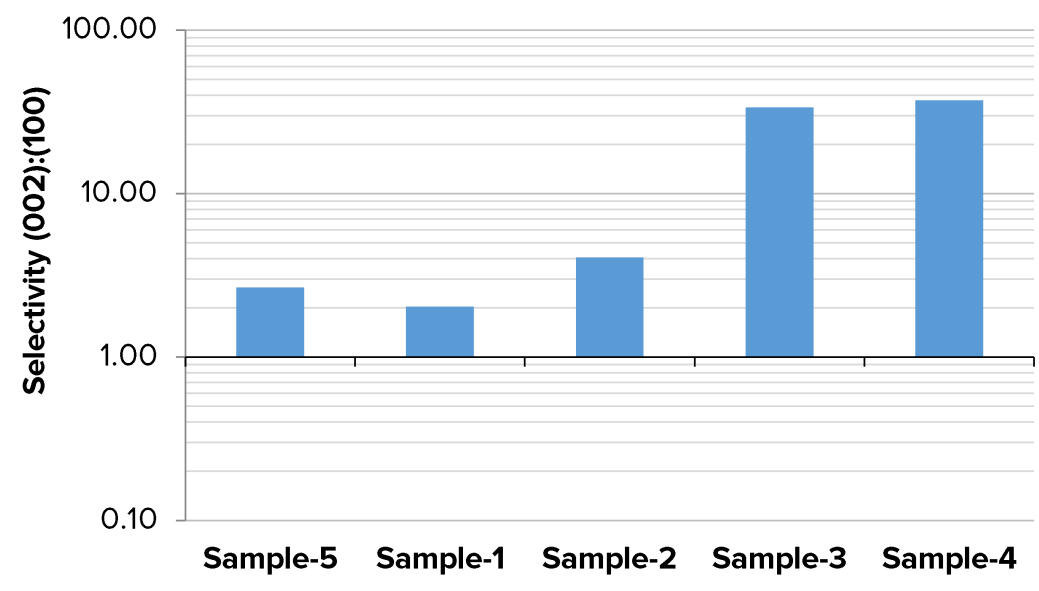

Figure 6.6: Noticeable improvement in the (002) selectivity when growing $\mathrm{ZnO}$ films directly on borosilicate.

\subsection{Sapphire}

$\mathrm{X}$-ray diffractograms of the different $\mathrm{ZnO}$ samples on top of sapphire substrates indicated that the sputtered buffer layers had a poor (002) selectivity, except for the case when only oxygen gas was used during the in situ annealing step. In addition, stress was almost eliminated and the FWHM values were almost unaffected by the in situ anneal step. 

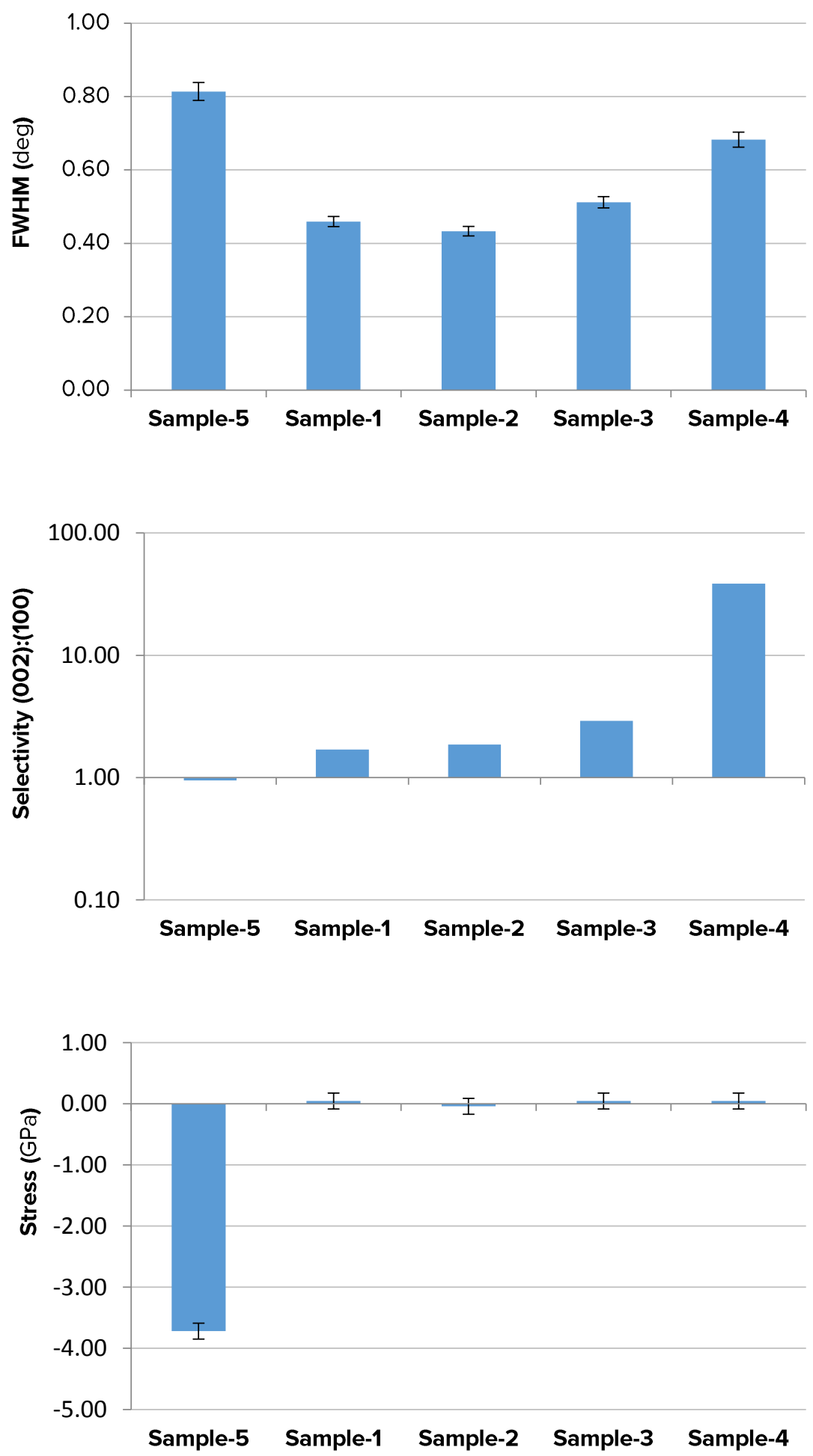

Figure 6.7: Plots showing the structural properties of the different $\mathrm{ZnO}$ samples on top of sapphire; top) FWHM, middle) $\mathrm{ZnO}$ (002):(100) selectivity, bottom) stress level 
These results were almost identical to the case of borosilicate glass, with a slightly better selectivity and lower stress levels in the case of sapphire substrates, as seen in figure 6.7.

As for borosilicate glass, certain regions of these sapphire samples were intentionally left out without a bottom contact structure, to allow direct $\mathrm{ZnO}$ deposition onto the sapphire substrate. X-ray diffractograms indicated a significant boost in (002) growth selectivity when $\mathrm{ZnO}$ was sputtered directly on to the substrate, as shown in figure 6.8. Again, the bottom metal contact had a significant detrimental effect on the (002) orientation selectivity.

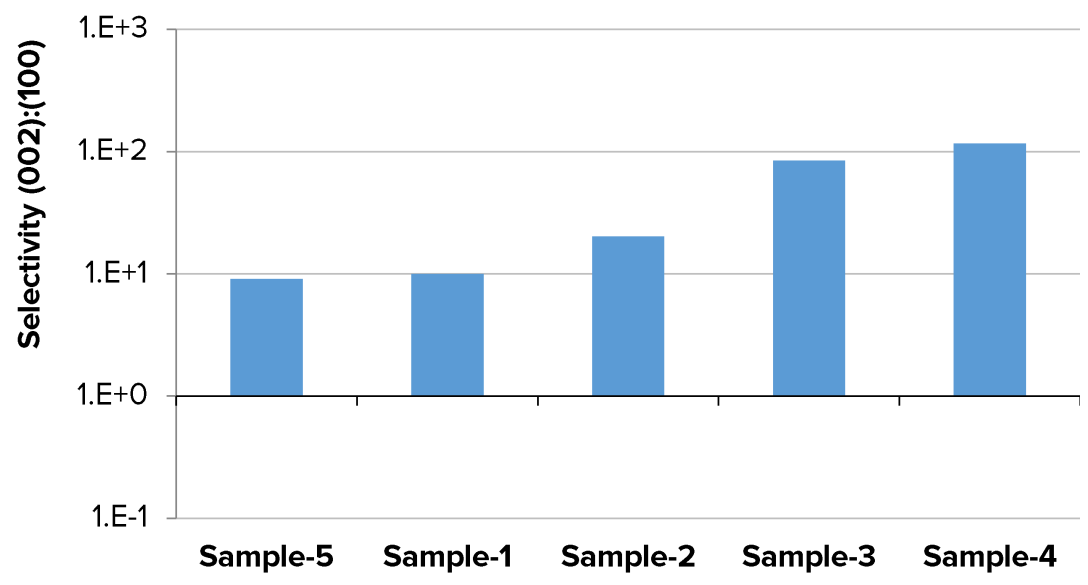

Figure 6.8: Noticeable improvement in the (002) crystal orientation selectivity when depositing $\mathrm{ZnO}$ directly onto sapphire.

\subsubsection{Electrical}

Electrical resistance measurements using the probe station showed that the buffer $\mathrm{ZnO}$ films on top of both borosilicate and sapphire substrates had a minimum resistivity of $500 \Omega . \mathrm{cm}$. Moreover, the results with respect to the benefits of having more oxygen in the in situ annealing gas mixture were found 
similar to the case of the soda-lime glass samples (section 6.3.1.2). Therefore, the same recommendations were deduced.

\subsubsection{Post-growth anneal of borosilicate and sapphire samples}

Samples from the in situ annealing study (section 6.3.2) were thermally annealed in the insulated furnace that was described in section 3.3. Understanding the changes that happen to $\mathrm{ZnO}$ films when annealed at elevated temperatures is crucial for the optimization of our fabrication process, as it is not possible to anneal at such temperatures inside the sputtering system used. Annealing is a significant processing step that can be used to optimize the electrical, structural, and electromechanical properties of our $\mathrm{ZnO}$ films, as mentioned in sections 3.3 and 6.3.

The parameters related to the external annealing system are:

- Annealing gas mixture, which is fixed at a 95\% $\mathrm{Ar}: 5 \% \mathrm{O}_{2}$

- Maximum annealing temperature, which could be adjusted using the furnace's microcontroller.

- Maximum annealing temperature duration, which was set to 2 hours.

- Temperature ramping rate, which was set to a fixed value of $+20^{\circ} \mathrm{C} / \mathrm{min}$.

- For the cooling part, the furnace was automatically shut down, and then allowed to cool down to ambient temperature.

Therefore, it can be deduced that the only variable to control during the external annealing step is the maximum annealing temperature. 
Thus for this study, annealing was carried out at a maximum temperature of $400^{\circ} \mathrm{C}$, and then consecutive annealing steps were carried out at higher temperatures, in steps of $200^{\circ} \mathrm{C}$, and up to $800^{\circ} \mathrm{C}$.

After each annealing process, the samples electrical resistance was characterized using the probe station described in section 4.3, while the structural properties were estimated using x-ray diffraction (section 4.2). This allowed us to examine the evolution of the electrical and structural properties of our $\mathrm{ZnO}$ films, as the annealing temperature increased.

In this study, it was found that the used borosilicate substrates did not withstand temperatures exceeding $400^{\circ} \mathrm{C}$, as they started to flow due to exceeding their glass temperature, rendering these samples useless. Consequently, this post-growth annealing study solely discusses the results of $\mathrm{ZnO}$ films deposited on top of sapphire substrates.

\subsubsection{Structural}

Residual $\mathrm{ZnO}$ film stresses were virtually eliminated on top of sapphire substrates after annealing at $400^{\circ} \mathrm{C}$, as seen in figure 6.9.

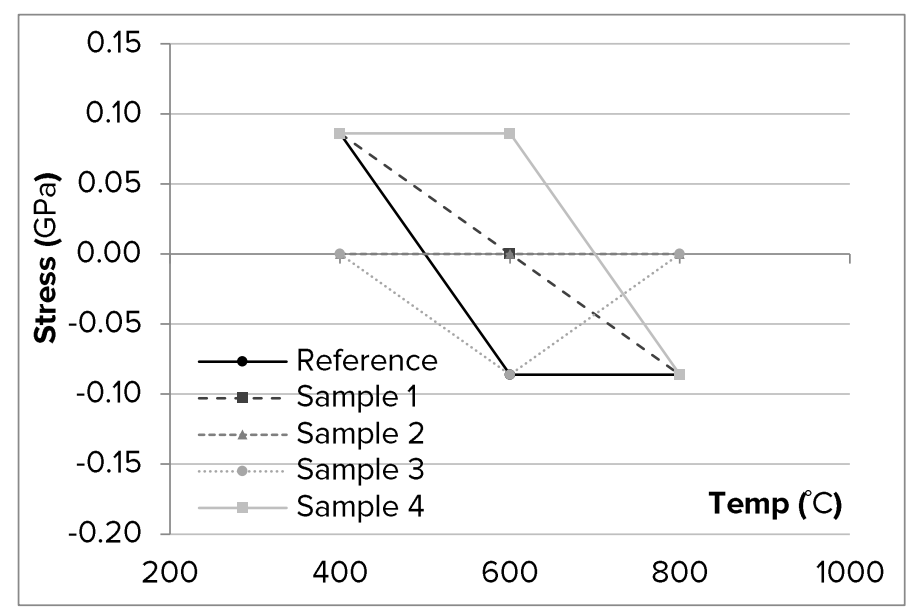

Figure 6.9: Stress for films deposited on sapphire versus the post growth annealing temperature. 
This result was also consistent for the $\mathrm{ZnO}$ films that were deposited directly onto the sapphire substrate.

On the other hand, as the annealing temperature increased, the FWHM of the $\mathrm{ZnO}$ (002) peak was found to significantly decrease, indicating a continuous increase in the average crystallite domain size (CDS), from about $5 \mathrm{~nm}$ at room temperature, to almost $50 \mathrm{~nm}$ at $800^{\circ} \mathrm{C}$, as seen in figure 6.10 . A similar trend was observed when the $\mathrm{ZnO}$ films were directly deposited onto the sapphire.

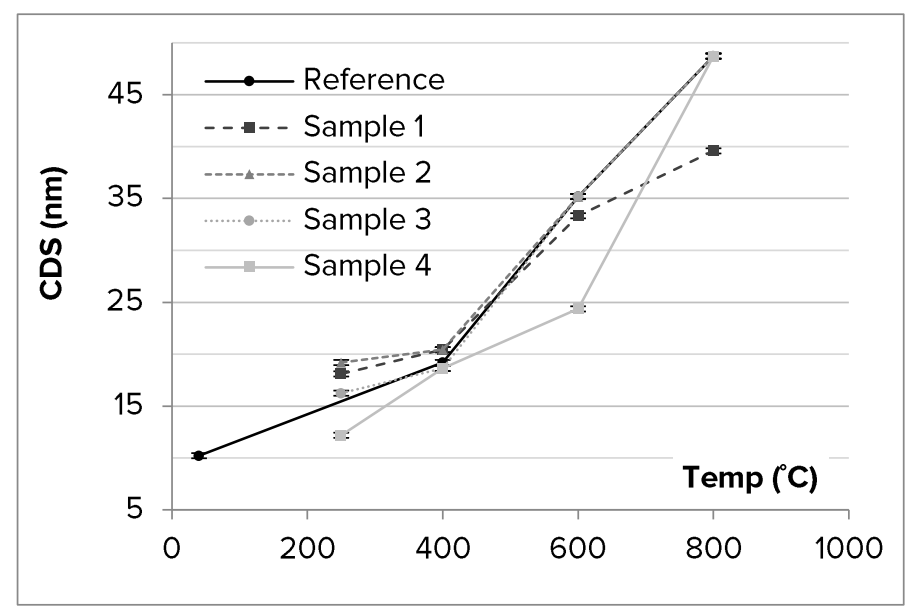

Figure 6.10: The estimated CDS of the $\mathrm{ZnO}$ (002) peaks for the different post-growth annealing temperatures.

Using more oxygen during the in situ annealing step meant having a higher value for the measured (002) peak maxima; this observation was maintained after external annealing, and directly resulted in having higher (002) selectivity values. For $\mathrm{ZnO}$ films grown directly on top of sapphire, the maximum (002) peak value exponentially increased as the annealing temperature increased; however, for the portions sputtered on top of the bottom metal contact, this trend was not observed, especially after annealing at $800^{\circ} \mathrm{C}$, with exception to the film that was in situ annealed in a 100\% oxygen background, as seen in 
figure 6.11. Depositing $\mathrm{ZnO}$ directly on the substrate meant having higher values for the (002) peak maximum, up to one order of magnitude. Therefore, (002) selectivity was found to drop on top of the metal contact, which signalled a significant problem regarding the prospects of growing c-axis $\mathrm{ZnO}$ films on top of the $\mathrm{Cr} / \mathrm{Au}$ contact structure, irrespective of the advantages offered by the sapphire substrate.

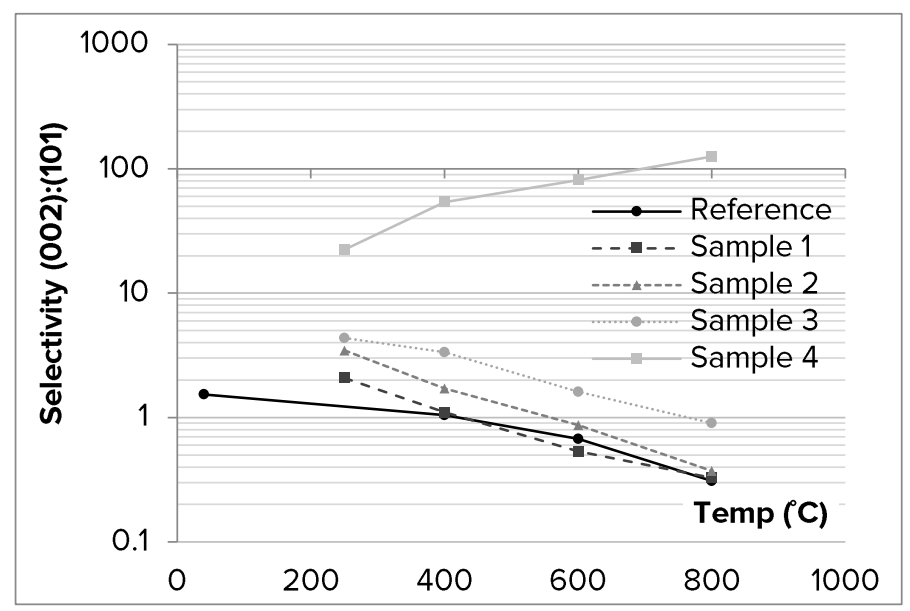

Figure 6.11: The estimated (002):(101) selectivity of the different $\mathrm{ZnO}$ films sputtered on top of c-axis sapphire as a function of the post-growth annealing temperature.

\subsubsection{Electrical}

At temperatures exceeding $600^{\circ} \mathrm{C}$, the metal contacts seemed to deteriorate and look transparent, probably due to them melting and diffusing, or due to agglomeration effects. This prevented further resistance measurements for the samples. Thus, no reliable resistance measurements were possible after annealing at such temperature, which indicate a catastrophic failure of such contacts when treated at high anneal temperatures. 


\subsection{Chapter Summary}

The results presented in this chapter confirmed the importance of having an oxygen-rich environment during the in situ anneal step of sputtered $\mathrm{ZnO}$ films, as it helps to achieve a lower stress, larger mean crystallite domain size, highly selective (002) texture, and high and reproducible electrical resistance. The following specific conclusions were reached:

- Lowering the relative oxygen concentration during the in situ anneal step causes the undesired $\mathrm{ZnO}$ crystal orientations to dominate.

- The inherent compressive stress within the as-grown $\mathrm{ZnO}$ films could be eliminated by annealing at a temperature of $400^{\circ} \mathrm{C}$, however anneal temperatures as high as $800^{\circ} \mathrm{C}$ are needed to ensure a dominant (002) textured film.

- It is suggested that the in-situ anneal in an oxygen atmosphere aids the elimination of oxygen vacancies, which are formed during the sputtering process. The elimination of these vacancies is essential to ensure having the desired (002) texture during recrystallization.

- The borosilicate substrate was found not suitable for high temperature anneal steps, as $600^{\circ} \mathrm{C}$ seemed to exceed its glass transition temperature.

- Selective growth along the (002) direction was found poor for all $\mathrm{ZnO}$ films grown on $\mathrm{Cr} / \mathrm{Au} / \mathrm{Cr}$ contacts. The only exception was the sapphire sample that received a one hour in situ anneal treatment in a $100 \%$ oxygen environment. 



\section{CHAPTER 7}

\section{Results C: Effects of Thermal Annealing on the Metal Contacts}

Based on the results reported in chapters 5 and 6, several questions remained unanswered when it came to optimizing the $\mathrm{ZnO}$ transducer fabrication process to create functional and thick piezoelectric $\mathrm{ZnO}$ films. Those questions mainly concerned the poor selectivity of the (002) crystal orientation of deposited $\mathrm{ZnO}$ on top of a metal $(\mathrm{Cr} / \mathrm{Au} / \mathrm{Cr})$ bottom contact (chapter 6).

Evidence in section 5.5.1 indicated that having a thinner bottom contact leads to an improved (002) selectivity, and section 6.3.3 reported having a better (002) selectivity when the $\mathrm{ZnO}$ was sputtered directly onto the substrate. Hence, the $\mathrm{Cr} / \mathrm{Au} / \mathrm{Cr}$ layer was found detrimental to the fabrication of proper device quality c-axis oriented $\mathrm{ZnO}$ layers. In addition, visual changes of the metal bottom contact layers where observed when the samples had undergone postgrowth thermal annealing; the films seemed to lose colour and become transparent as the annealing temperature became higher. Thus, it was suspected 
that the contacts were being damaged, as it is known that thermal annealing can disintegrate metal thin-films into particles by the process of dewetting [210, 211, 212]. This structural degradation is driven by the reduction of the surface and strain energy [213], and is known to take place at temperatures well below the melting point of the metal (for gold $T_{m}=1064^{\circ} \mathrm{C}[212]$ ), so that a film remains in the solid-state [214]. Thus, the required post-growth thermal annealing process is expected to have both positive effects on the active $\mathrm{ZnO}$ layer, and negative effects on the metal contacts.

\subsection{Chapter Objectives}

This chapter describes a study that was conducted to evaluate the effects of postgrowth external annealing at elevated temperatures on the quality of the $\mathrm{Cr} / \mathrm{Au} / \mathrm{Cr}$ contacts. The study also compares the effects of the in situ annealing conditions on the properties of the resulting metal and $\mathrm{ZnO}$ films. Only sapphire substrates were considered in this work. In addition, this study uniquely provides insights into the microstructural evolution of both bare metal films, and metal films capped with a thicker RF-magnetron sputtered $\mathrm{ZnO}$ layer, as the annealing temperature increased.

\subsection{Microstructure Evolution of Metal/ZnO Films}

This study was initiated to investigate the limits of film optimization of such $\mathrm{Cr} / \mathrm{Au} / \mathrm{Cr} / \mathrm{ZnO}$ structures by means of in situ and post-growth furnace thermal annealing, and to evaluate the quality of the metal contact structure as a function of the annealing temperature, to examine the compatibility of metal films with this essential structure optimization step. This study was also meant 
to examine the evolution of the morphology, crystal properties, and electrical properties of identical $\mathrm{ZnO}$ films grown on top of both the substrate, and a 20nm thin gold layer, as the annealing temperature was increased.

XRD, AFM, SEM, EDS and RF impedance measurements were carried out to examine the structural evolution of thin $\mathrm{Cr} / \mathrm{Au} / \mathrm{Cr}$ films deposited on top of sapphire substrates, as the annealing temperature was increased from $250^{\circ} \mathrm{C}$ to $800^{\circ} \mathrm{C}$.

\subsubsection{Fabrication}

The transducer fabrication process was started by depositing a $\mathrm{Cr}(1 \mathrm{~nm}) / \mathrm{Au}$ $(20 \mathrm{~nm}) / \mathrm{Cr}(1 \mathrm{~nm})$ bottom contact on half of the substrate, while the other half was left bare to evaluate the effects of having a bottom metal contact on the deposited $\mathrm{ZnO}$ layer. Consequently, a 250nm thick $\mathrm{ZnO}$ layer was sputtered at an RF power of $150 \mathrm{~W}$ in an Ar gas background at a pressure of $\sim 1.5 \times 10^{-2}$ mbar.

After this deposition, the films were subjected to an in situ anneal inside the sputtering chamber using a substrate quartz lamp heater. This step was done at $250^{\circ} \mathrm{C}$ in an $\mathrm{Ar}: \mathrm{O}_{2}$ gas mixture for 1 hour. The heating rate was $\sim 3.3^{\circ} \mathrm{C} / \mathrm{min}$, and the temperature set point was held for 1 hour. The samples were allowed to cool in the same gas mixture. Based on the results of the previous chapter, in situ annealing was found to favour the growth and recrystallization of the (002) orientation, where this desired (002) orientation became more dominant as the $\mathrm{O}_{2}$ gas content increased during annealing. The trend indicated an exponentially better selectivity when increasing the oxygen content. This improvement can be attributed to the combination of two effects: when $\mathrm{ZnO}$ is sputtered in pure argon, the resulting films tend to be $\mathrm{Zn}$-rich 
with poor c-axis alignment [215, 216]. On the other hand, the $\mathrm{ZnO}(002)$ orientation is the most closely packed with the lowest free-surface-energy. Thus in a thin wurtzite $\mathrm{ZnO}$ film, the (002) orientation is preferred [205], unless oxygen vacancies are introduced during the process. We therefore suggest that such oxygen vacancies are reduced during annealing in an oxygen-rich gas mixture. To test this theory, two different in situ annealing gas-mixture compositions were used in this study, while a reference substrate was prepared without the in situ annealing step:

- Substrate-1: annealing gas mixture was 100\% Ar.

- Substrate-2: annealing gas mixture was $100 \% \mathrm{O}_{2}$.

- Reference substrate: no in situ annealing step.

Each substrate was then removed from the sputtering chamber and divided into 8 different samples (Figure 7.1), 4 having $\mathrm{ZnO}$ directly deposited onto sapphire, and 4 having $\mathrm{ZnO}$ deposited on top of $\mathrm{Cr} / \mathrm{Au} / \mathrm{Cr}$ layers. Thus, the processing steps resulted in 24 different samples. 


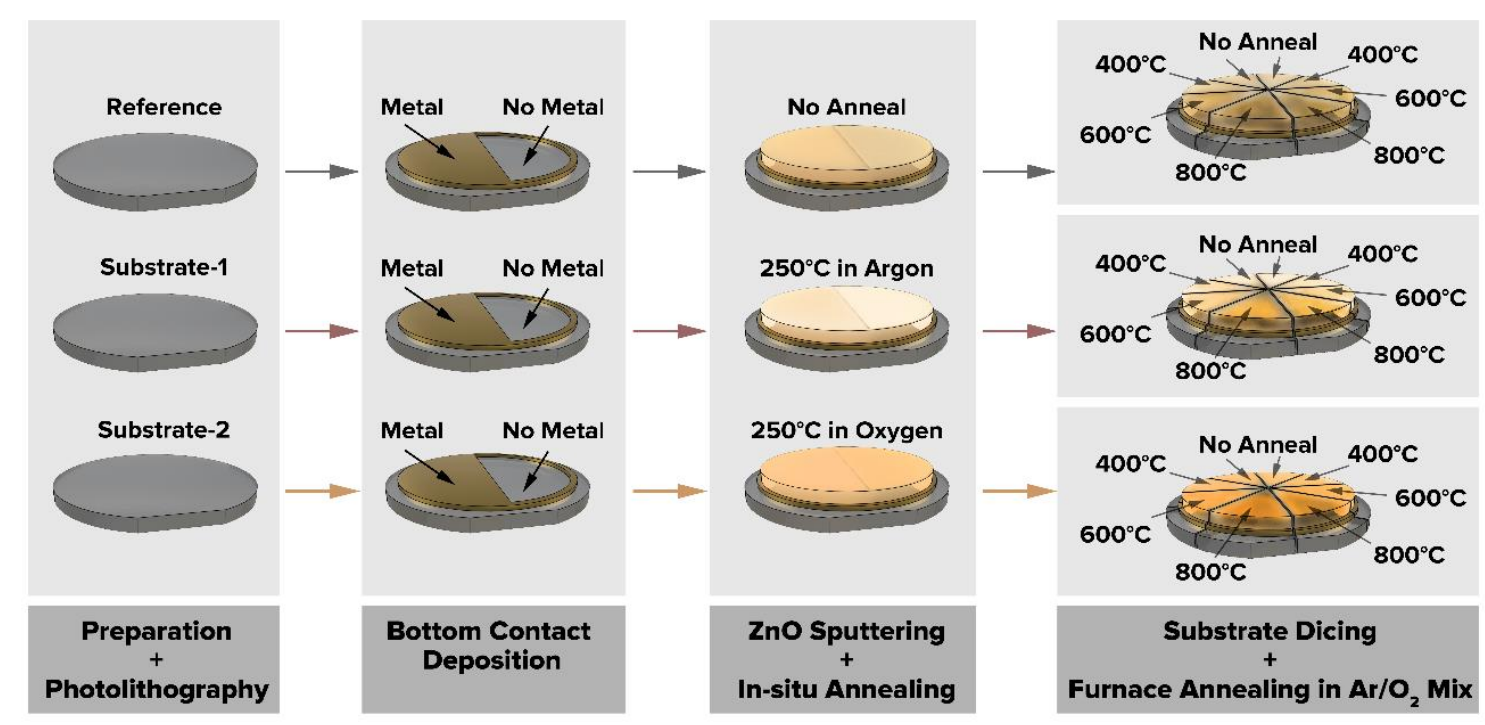

Figure 7.1 A sketch highlighting the different fabrication steps involved in creating the 24 samples involved in this study.

A tube furnace was used to carry a final post-growth anneal for each of the 24 samples ( 3 substrates $\times 8$ samples per substrate). This external annealing step was carried out in a $95 \% \mathrm{Ar}: 5 \% \mathrm{O}_{2}$ gas flow, with a holding time of 2 hours at the highest temperature. The heating rate was $20^{\circ} \mathrm{C} / \mathrm{min}$, and samples were left to cool in the same gas mixture. Three different temperature set points $T_{A}$ of $400^{\circ} \mathrm{C}, 600^{\circ} \mathrm{C}$, and $800^{\circ} \mathrm{C}$ were used, while the fourth sample in each halfsubstrate was left un-annealed to provide a control for measurements.

\subsubsection{SEM}

Imaging was done at a low voltage using the secondary electron detector to examine the topography, grain boundaries, and facets at the films' surfaces. Additional imaging was done using a high voltage electron beam and a backscattered electron detector to examine the bulk nature of the films, and to observe the dewetting progress of the metal films as a function of the annealing 
temperature. An energy dispersive X-ray spectrometer (EDS) was used in combination to confirm the compositional identity of the dewetting films.

\subsubsection{Low Voltage SEM}

To produce low voltage SEM micrographs that are sensitive to sample topography, electrons with $3 \mathrm{keV}$ energy were used for imaging, and the secondary electron detector was used. The morphological examination presented in figure 7.2 revealed that the $\mathrm{ZnO}$ film grains became larger as the annealing temperature increased. At $800^{\circ} \mathrm{C}$, the grains appeared faceted. This observation suggests that the grains were in the solid state during thermal equilibration, similar to the case reported by Sadan et alia [212].

\section{Reference Substrate}

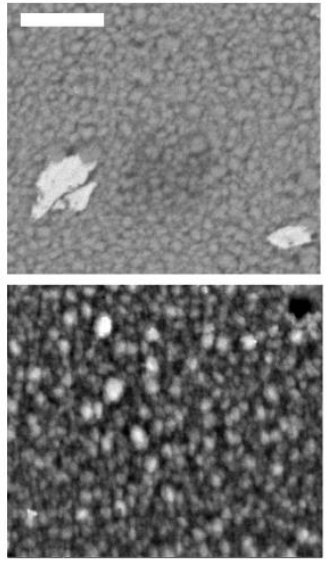

As Sputtered

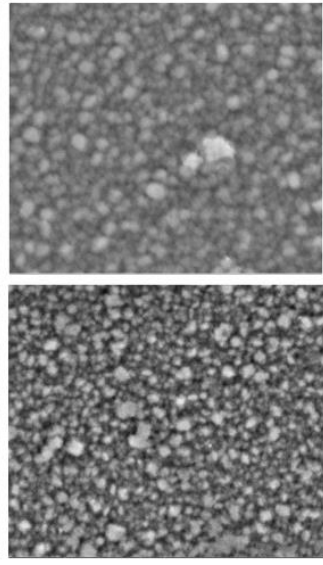

$400^{\circ} \mathrm{C}$
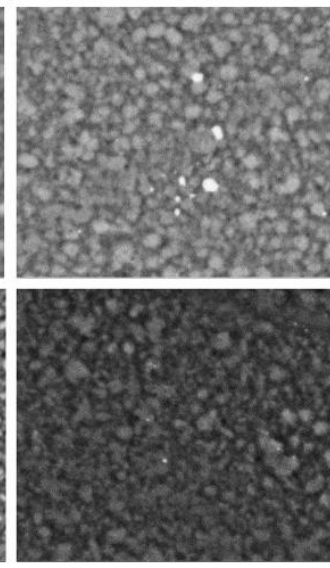

$600^{\circ} \mathrm{C}$

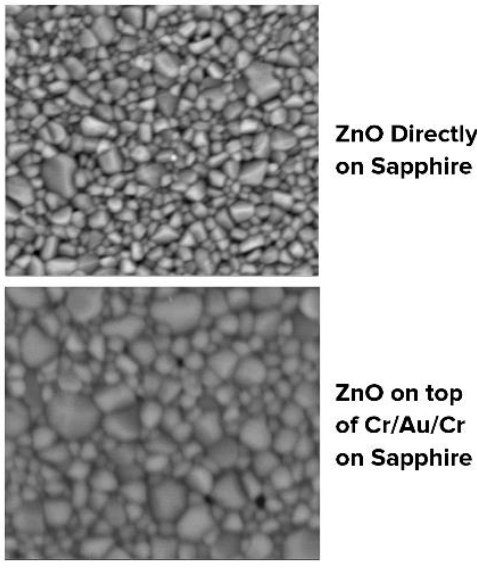

$800^{\circ} \mathrm{C}$

External Annealing Temperature

Figure 7.2: Top-view secondary electrons SEM micrographs of $\mathrm{ZnO}$ films, showing morphology changes as the annealing temperature increased. The scale bar equals $500 \mathrm{~nm}$ and is equivalent for all micrographs.

The micrographs indicate a growth in the grain diameter $\varphi_{\operatorname{gr}(Z n O)}$ from $\sim 50 \mathrm{~nm}$ (as sputtered) to $\sim 150 \mathrm{~nm}$ after annealing at $800^{\circ} \mathrm{C}$. $\mathrm{ZnO}$ sputtered directly on 
sapphire appears to have a slower and less symmetric grain growth with $\varphi_{\operatorname{gr}(\mathrm{ZnO})} \sim 90 \mathrm{~nm}$. There were no significant differences when comparing all three substrates under such imaging conditions.

\subsubsection{High Voltage SEM}

Increasing the accelerating voltage of the electron beam used for imaging increases the depth of the electronic interaction profile within the sample [217], where electrons having energies of $15-20 \mathrm{keV}$ are expected to have a penetration depth exceeding $1 \mu \mathrm{m}[217,179]$; this enables imaging through the different layers of our samples, and allows energy dispersive X-ray spectroscopy.

By using the backscattered electron detector, we were able to obtain micrographs that contained information about the grain boundaries of both $\mathrm{ZnO}$ and gold films, as well as the elemental composition at the different areas of the sample. This enabled imaging the different stages of the dewetting process, as the relatively large gold atom yields more backscattered electrons, thus appearing brighter than $\mathrm{ZnO}$ and sapphire in the micrographs [179].

Based on figure 7.3, the as-deposited films appear intact, and no voids or defects are observed after the in situ annealing step at $250^{\circ} \mathrm{C}$. Void initiation and growth in a branched manner can be seen after the samples were annealed at $400^{\circ} \mathrm{C}$, as darker patches indicating a lack of gold become visible. This branched or fractal void growth mechanism has been described in literature [218], and is characterized by elongated void features that violate the minimum void perimeter preference associated with the capillary process [214]. Void diameters $\varphi_{\text {void }}$ were in the $1-8 \mu \mathrm{m}$ range for all three substrates. 


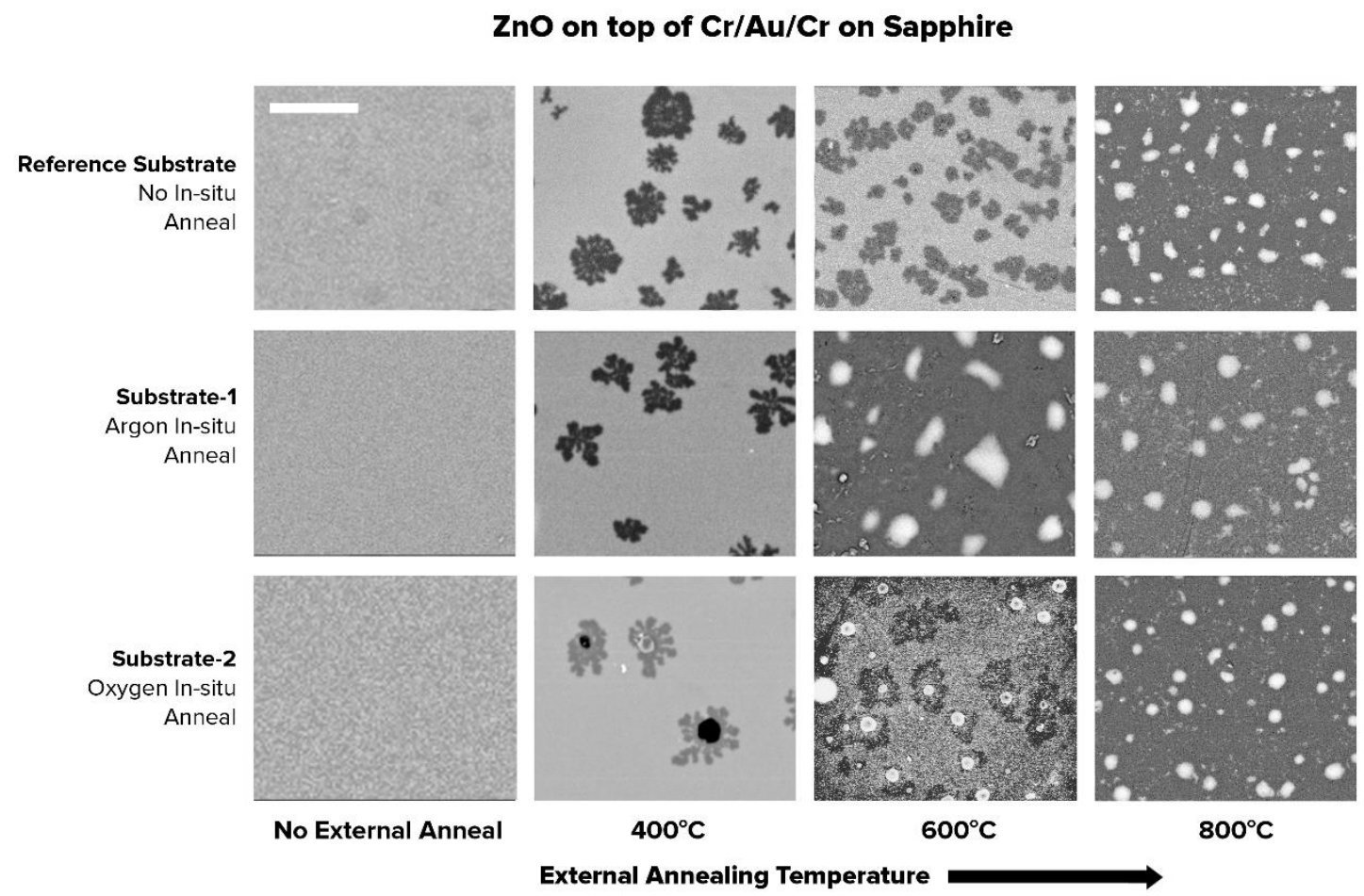

Figure 7.3: Top-view backscattered electron SEM micrographs of $\mathrm{ZnO}$ films, showing gold dewetting as the annealing temperature increased. The scale bar equals $10 \mu \mathrm{m}$ and is equivalent for all micrographs.

The sample from substrate-2, which had a previous pure oxygen in situ anneal, had two different contrast regions in its void regions: a circular central core, and a branched outer area, possibly indicating a slower dewetting progress.

As the annealing temperature is raised to $600^{\circ} \mathrm{C}$, void growth is seen to proceed. Such void growth appears to progress at different rates for each sample, being fastest for the sample from substrate-1, with an almost total agglomeration with separate islands of gold, and slowest for the sample from substrate-2. Coalescence was dominant after annealing at $800^{\circ} \mathrm{C}$, resulting in gold agglomerated particles having an average diameter $\varphi_{\text {gold }} \sim 1.5 \mu m$. 
As the $\mathrm{ZnO}$ layer was sputtered on a smaller circular area than the bottom $\mathrm{Cr} / \mathrm{Au} / \mathrm{Cr}$ contact, there were areas in each of our samples that had an exposed contact surface. By examining the SEM micrographs of such areas (figure 7.4), and comparing them with the previous results (figure 7.3), we could infer a measure of the protective effect against gold dewetting due to having a $250 \mathrm{~nm}$ thick $\mathrm{ZnO}$ layer on top.

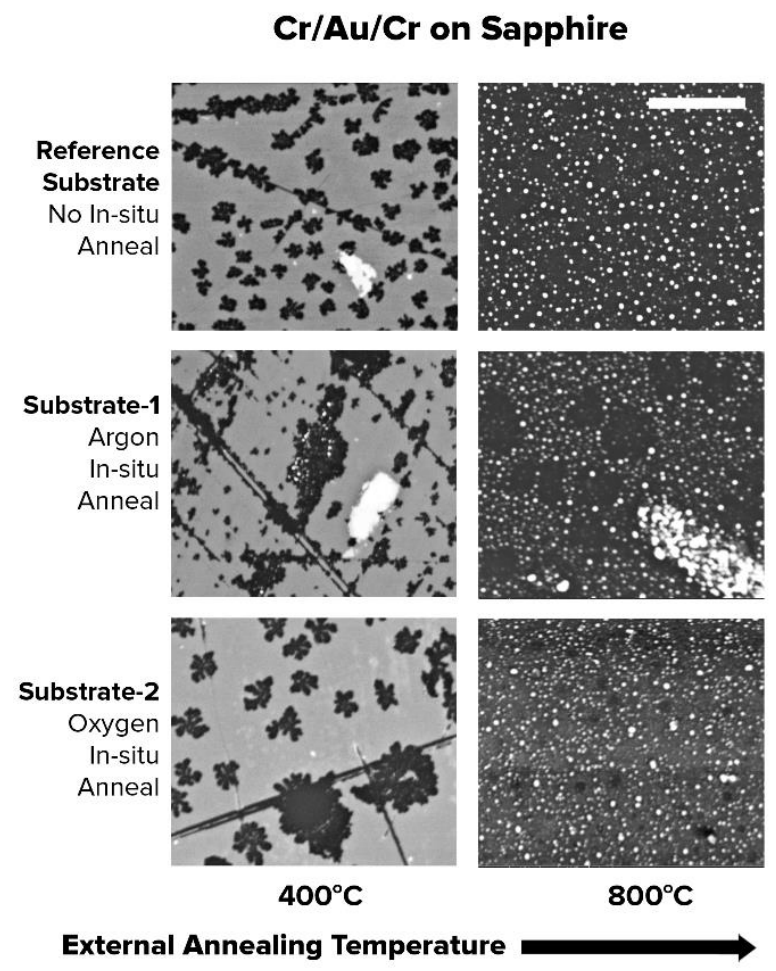

Figure 7.4: Top-view backscattered electron SEM micrographs of $\mathrm{Cr} / \mathrm{Au} / \mathrm{Cr}$ films showing dewetting as the annealing temperature is increased. The scale bar equals $10 \mu \mathrm{m}$ and is equivalent for all micrographs.

Our SEM micrographs indicated that the contact films were intact as deposited, and even after in situ annealing at $250^{\circ} \mathrm{C}$. However, dewetting proceeded in a faster manner relative to the previously shown case where a $\mathrm{ZnO}$ capping layer existed. After annealing at $400^{\circ} \mathrm{C}$, void initiation and growth is observed for 
samples from all substrates, with void diameters $\varphi_{\text {void }}$ in the $1-15 \mu \mathrm{m}$ range. Increasing the annealing temperature to $800^{\circ} \mathrm{C}$ resulted in smaller gold particles with an average diameter $\varphi_{\text {gold }} \sim 500 \mathrm{~nm}$.

\subsubsection{EDS}

To confirm the compositional identity of the observed voids and agglomerates at the different annealing temperatures, energy dispersive X-ray spectroscopy (EDS) mapping was used, as shown in figure 7.5. Given the non-conductive nature of our $\mathrm{ZnO}$ films, carbon coating was applied to minimize charging related drift and allow long acquisition times necessary for EDS mapping.

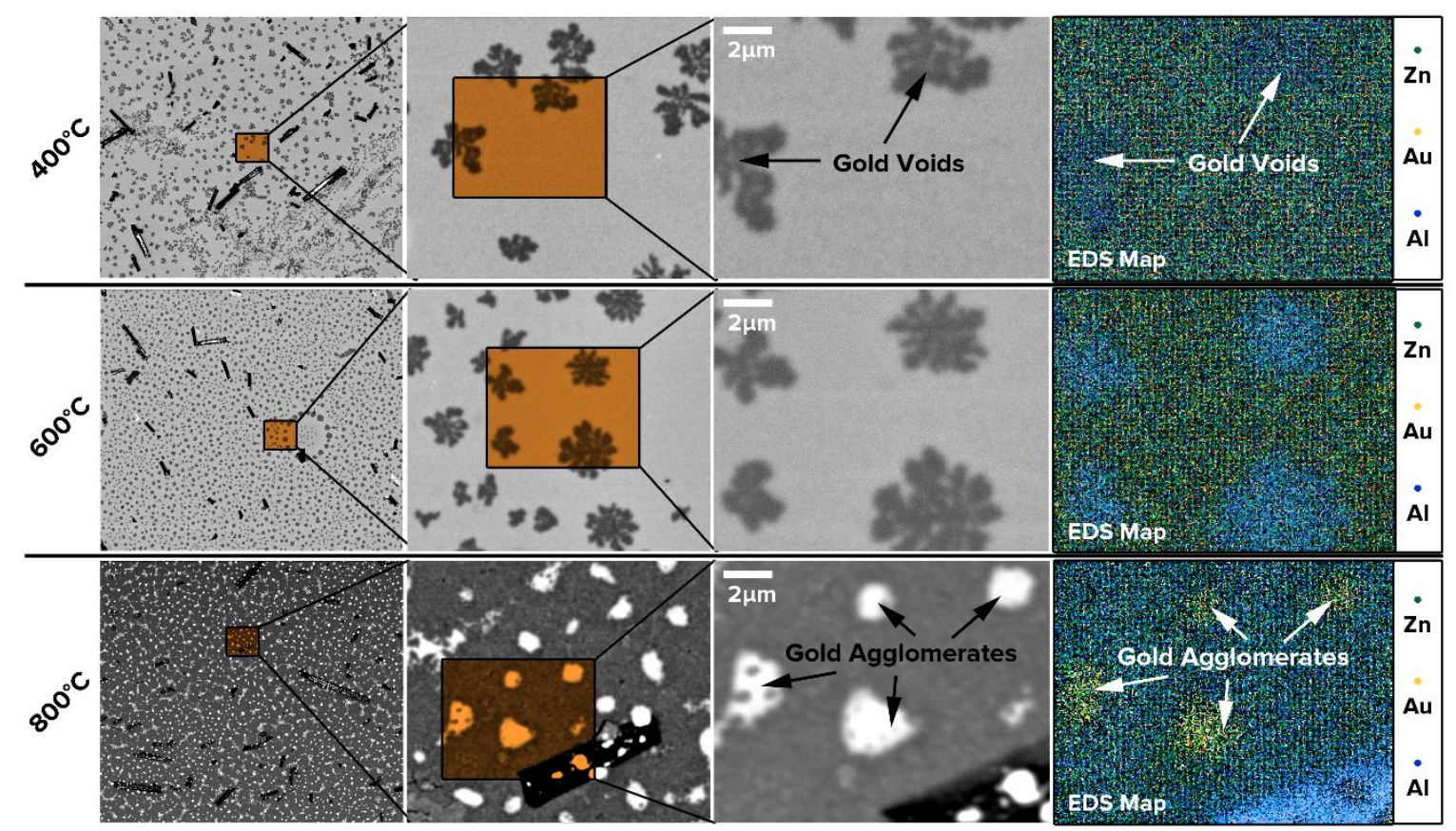

Figure 7.5: Top-view backscattered electron SEM micrographs of $\mathrm{ZnO}$ films showing dewetting of the gold contact underneath as annealing progressed from $400^{\circ} \mathrm{C}$ to $800^{\circ} \mathrm{C}$. The right column shows the overlaid EDS maps of the adjacent imaged areas, with the label indicating each element's colour map. 
Elements used for spectrum identification were: $\mathrm{Al}, \mathrm{O}$ for sapphire; $\mathrm{Cr}, \mathrm{Au}$ for the bottom metal contact; and $\mathrm{Zn}, \mathrm{O}$ for the $\mathrm{ZnO}$ film. Given the relative low frequency of X-rays emitted from the oxygen's K-level [219], and the small amount of $\mathrm{Cr}$ in our samples, spectrum analysis focused on identifying three elements: $\mathrm{Al}$ (sapphire), $\mathrm{Au}$ (bottom contact) and $\mathrm{Zn}$ ( $\mathrm{ZnO}$ film). Comparing the SEM micrographs with their corresponding overlaid EDS maps, it is clear that the darker regions observed after annealing at $400^{\circ} \mathrm{C}$ are indicative of gold voids. These voids became larger and more abundant after the samples got annealed at $600^{\circ} \mathrm{C}$. Finally, annealing at $800^{\circ} \mathrm{C}$ reduced the gold film into micron sized particles, thereby confirming the $\mathrm{Cr} / \mathrm{Au} / \mathrm{Cr}$ film dewetting process. EDS maps indicated no changes in the Zn contents (green map) as the annealing temperature increased.

\subsubsection{Combining Low and High Voltage SEM}

$\mathrm{ZnO}$ grain growth and morphology changes at $800^{\circ} \mathrm{C}$ for the three substrates were compared on top of both sapphire and $\mathrm{Cr} / \mathrm{Au} / \mathrm{Cr}$-on-sapphire, by combining both low voltage (secondary electron detector) and high voltage (backscattered electron detector) SEM micrographs, as seen in figure 7.6.

The first column of micrographs (left) was imaged using a high voltage tension of $18 \mathrm{kV}$ and the backscattered electron detector, showing areas where the gold contact layer still existed after dewetting. The $\mathrm{ZnO}$ grains are visible in an overlaid manner in these micrographs, and show no morphological changes or grain size variations with regards to the existence of gold particles underneath. 


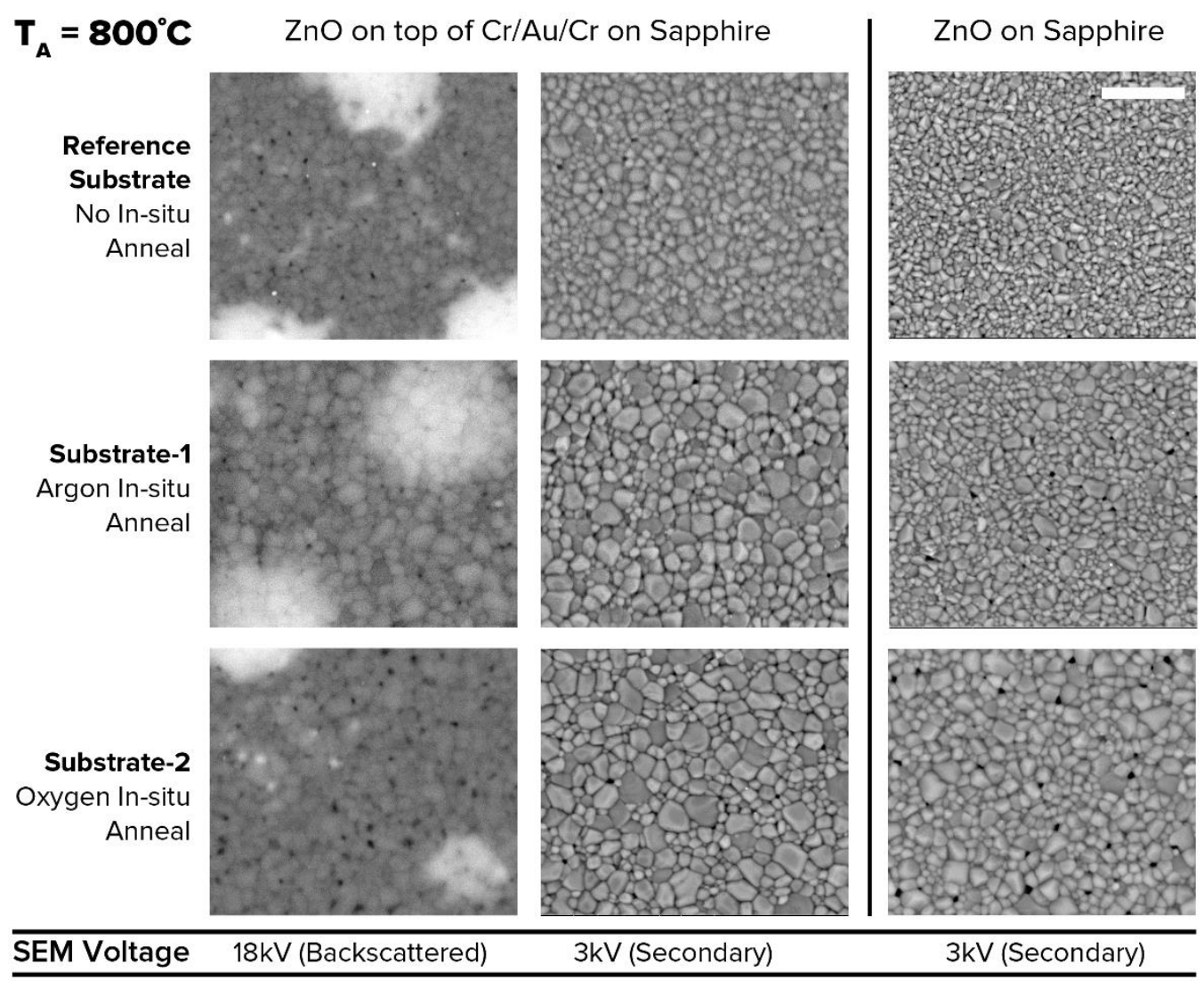

Figure 7.6: Top-view SEM micrographs of $\mathrm{ZnO}$ films after annealing at $800^{\circ} \mathrm{C}$. The voltage used for imaging and the selected detector type are shown for each column. The scale bar equals $500 \mathrm{~nm}$ and is equivalent for all micrographs.

The other two columns of micrographs (middle and right) are imaged using a low voltage tension of $3 \mathrm{kV}$ and the secondary electron detector. These micrographs show a comparable amount of $\mathrm{ZnO}$ grain growth for all three substrates, with a slight increase for the sample from substrate-1. The average grain diameter is estimated at $\varphi_{\operatorname{gr}(\mathrm{ZnO})} \sim 150 \mathrm{~nm}$. Again, $\mathrm{ZnO}$ sputtered directly on sapphire appears to have a slower and less symmetric grain growth with an average grain diameter $\varphi_{\operatorname{gr}(\mathrm{Zno})} \sim 90 \mathrm{~nm}$. 


\subsubsection{Atomic Force Microscopy}

AFM images were taken to provide a quantitative measure of the samples' surface variations as the annealing temperature increased. The minimum height measured was offset to zero in our scans. Figure 7.7 reveals the metal contact dewetting progress with respect to the annealing temperature, where voids become visible after annealing at $400^{\circ} \mathrm{C}$. Dewetting is seen to progress at $600^{\circ} \mathrm{C}$, and complete agglomeration occurs at $800^{\circ} \mathrm{C}$.

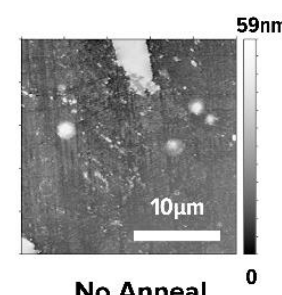

No Anneal

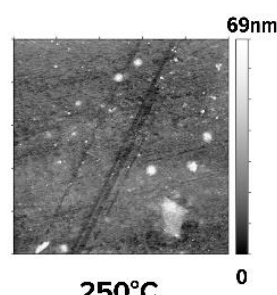

$250^{\circ} \mathrm{C}$

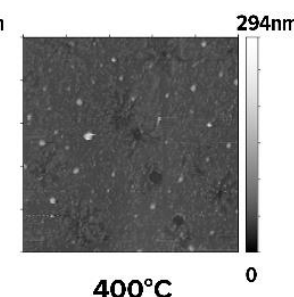

$400^{\circ} \mathrm{C}$

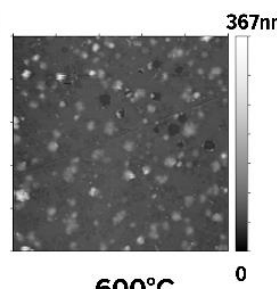

$600^{\circ} \mathrm{C}$

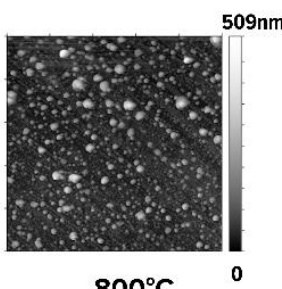

$800^{\circ} \mathrm{C}$
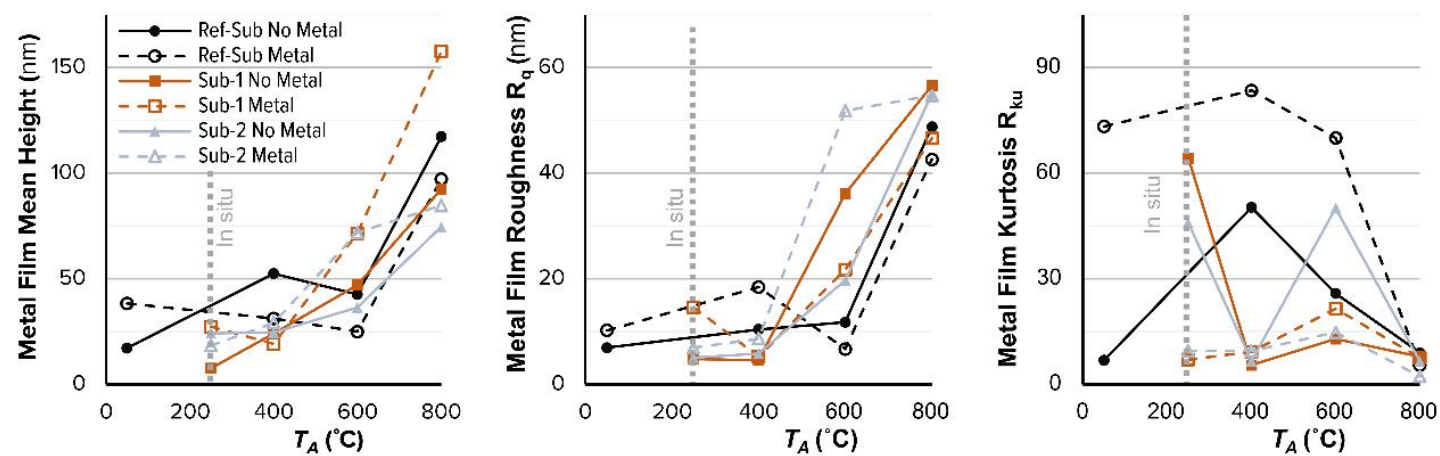

Figure 7.7: AFM images of the exposed metal contact as the annealing temperature was increased. The scale bar equals $10 \mu \mathrm{m}$ and is equivalent for all images. The lower graphs show the mean height, roughness and kurtosis of the metal surfaces in all samples.

The mean height of the metal contact films for different samples increased when the annealing temperature was raised. The sharpest increase was observed after annealing at $800^{\circ} \mathrm{C}$. Similarly, the film roughness $R_{q}$ increased 
from $\sim 10 \mathrm{~nm}$ at $T_{A} \leq 250^{\circ} \mathrm{C}$ up to $\sim 50 \mathrm{~nm}$ at $800^{\circ} \mathrm{C}$. While the annealing temperature increased, the kurtosis of the different samples converged to values close to zero, indicating uniform film agglomeration with a normal distribution for the resulting particle size.

As to $\mathrm{ZnO}$ films, AFM imaging confirmed the grain growth as the annealing temperature increased, as shown in figure 7.8.
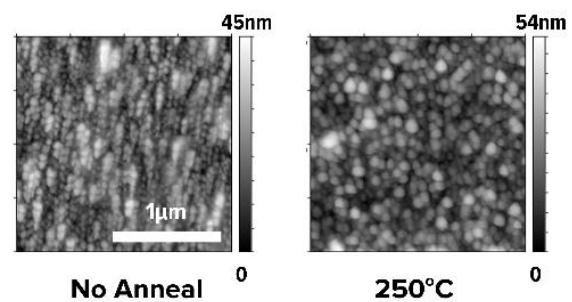

$250^{\circ} \mathrm{C}$
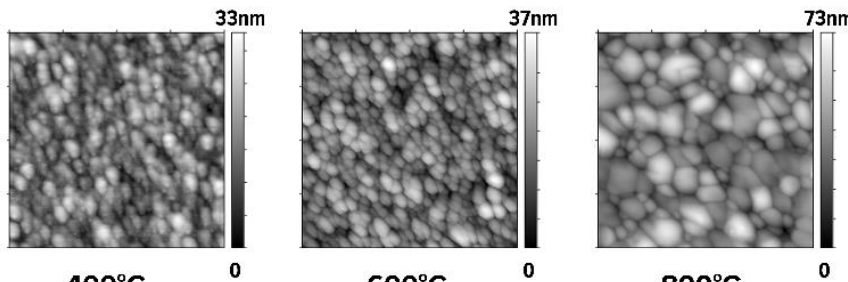

Annealing Temperature

$800^{\circ} \mathrm{C}$

Figure 7.8: AFM images showing the grains of the $\mathrm{ZnO}$ films as the annealing temperature increased. The scale bar equals $1 \mu \mathrm{m}$ and is equivalent for all images.

$\mathrm{ZnO}$ grain growth was faster and larger when the bottom metal layer existed, seen in figure 7.9. The grains grew from $\sim 55 \mathrm{~nm}$ as sputtered to $\sim 95 \mathrm{~nm}$ on top of sapphire and $\sim 147 \mathrm{~nm}$ with the metal underlayer after annealing at $800^{\circ} \mathrm{C}$, i.e., it was consistent with the SEM results. 


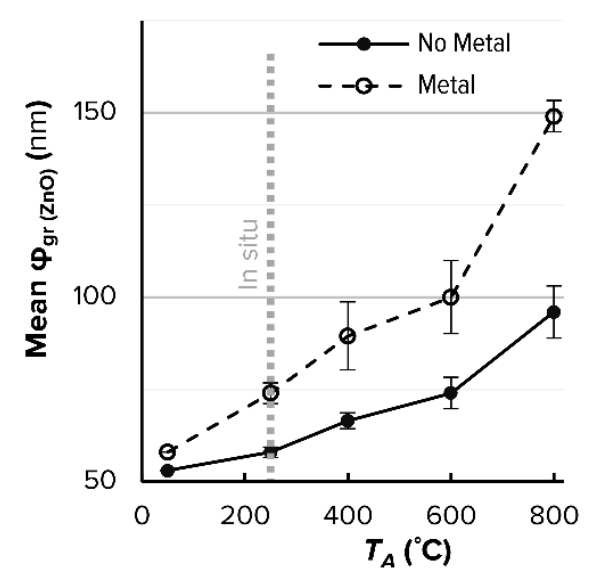

Figure 7.9: AFM measurements plot showing $\mathrm{ZnO}$ grain growth due to annealing for samples with and without the metal underlayer.

\subsubsection{X-Ray Diffraction}

\subsubsection{ZnO Films}

\subsection{Stress}

Measurements of the samples from the reference substrate indicated that $\mathrm{ZnO}$ is slightly more compressed when deposited directly on the substrate than when a bottom metal layer existed (figure 7.10). The same result was observed in the annealing studies of chapter 6 . However, as the bottom contact was thicker in the annealing study (50nm gold), it was found that reducing the gold thickness (20nm now) reduces the stress by $36 \%$ on top of the metal. 


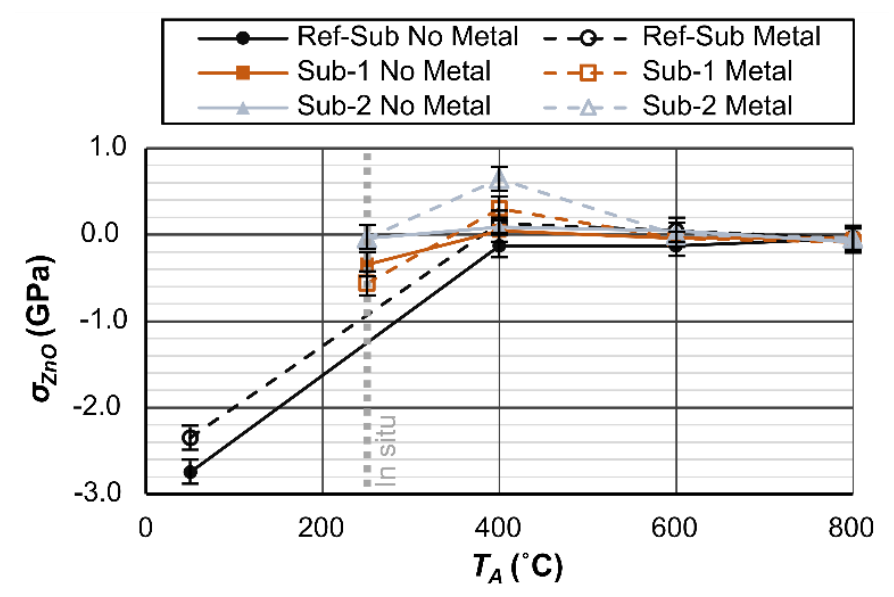

Figure 7.10: The estimated stress of the $\mathrm{ZnO}$ films in all samples as a function of the annealing temperature.

For samples from substrate-2, the in situ annealing step was found sufficient to eliminate the inherent $\mathrm{ZnO}$ film stress. As to samples from substrate-1, a 0.5GPa compressive stress remained in the films after the in situ annealing step. For $\mathrm{T}_{\mathrm{A}}$ $\geq 600^{\circ} \mathrm{C}$, the stress values converged to null for all substrates, settling at $0.08 \mathrm{GPa}$, a result that is very similar to the annealing study of the previous chapter.

\subsection{Selectivity}

The (002):(100) orientation selectivity was significantly better (200\% to $400 \%$ ) when the $\mathrm{ZnO}$ was deposited on the metal layer rather than directly on sapphire, as seen in figure 7.11. This result is opposite to the previous annealing study, where sputtering directly on sapphire gave a $1000 \%$ improvement in selectivity, compared to when sputtering on the metal. However, the thickness of the $\mathrm{ZnO}$ layer was 3 times more in the previous study $(750 \mathrm{~nm})$, and the metal layer was 2.5 times thicker (50nm), which renders the comparison unfair. 


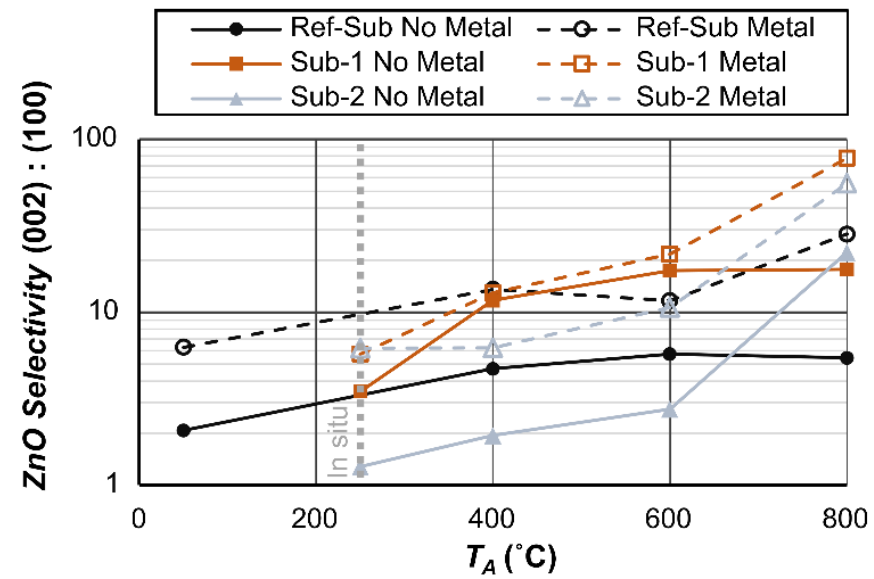

Figure 7.11: The deposited $\mathrm{ZnO}$ (002):(100) selectivity for all samples as obtained from the reflections in the XRD diffractograms.

Previously, it was found that having more oxygen in the in situ annealing step improved this selectivity. However, no relevant differences can be spotted in this study, where using oxygen gave slightly worse selectivity than Ar. It should be noted that the rate of selectivity improvement was fastest (exponential) for samples having $\mathrm{ZnO}$ deposited on the metal layer in the 600$800^{\circ} \mathrm{C}$ annealing temperature range. This is possibly due to the disintegration of the underlying metal layer. Selectivity improvement due to annealing was lower for $\mathrm{ZnO}$ films deposited on sapphire, where the highest improvement was seen for samples from substrate-2 (oxygen in situ).

\subsection{Crystallite Domain Size}

All samples indicated an exponential increase in the $\mathrm{ZnO}$ (002) crystallite domain size (CDS) as the annealing temperature increased, as shown in figure 7.12 . 


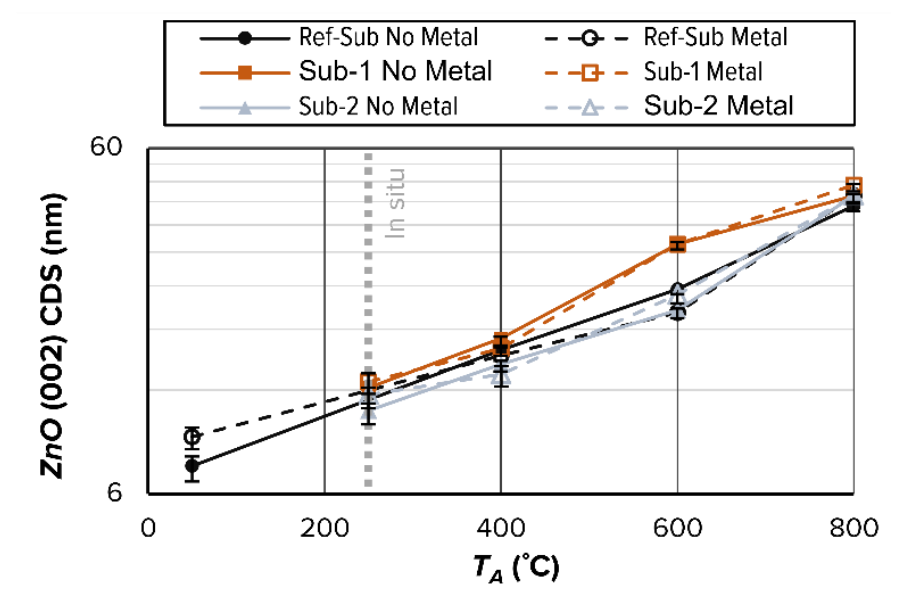

Figure 7.12: The estimated $\mathrm{ZnO}$ (002) crystallite domain size for the different samples as a function of the annealing temperature.

The reference samples that had no annealing showed a CDS of $7 \mathrm{~nm}$ when deposited on sapphire, and 9nm when the bottom metal layer existed. The CDS was slightly larger for $\mathrm{ZnO}$ films sputtered on top of the metal layer rather than directly onto sapphire. Samples from substrate-2 (oxygen in situ) had the smallest CDS. This result is resonant with the results achieved in the annealing studies of the previous chapter, where adding more oxygen resulted in smaller CDS. However, after annealing at $800^{\circ} \mathrm{C}$, all samples were comparable in terms of their CDS (41-45nm), which is around $18 \%$ of the $\mathrm{ZnO}$ film thickness. This improvement in CDS indicates that our $\mathrm{ZnO}$ films became increasingly columnar as the annealing temperature increased.

\subsubsection{Gold Films}

\subsection{Crystallite Domain Size}

The gold film had a dominant $\mathrm{Au}$ (111) orientation with a mean crystallite domain size of $6 \mathrm{~nm}$ after deposition, as shown below in figure 7.13. 


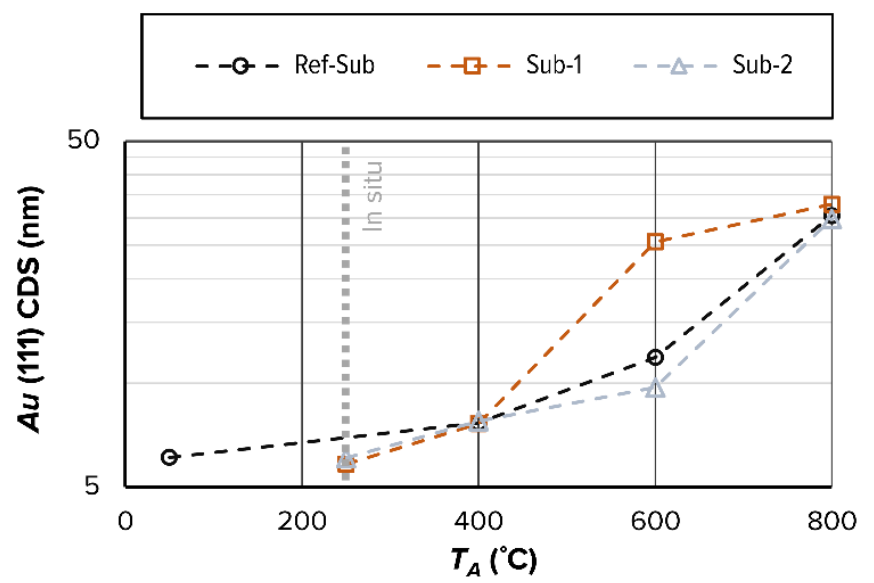

Figure 7.13: The estimated crystallite domain size of $\mathrm{Au}$ (111) for the different samples as a function of the annealing temperature.

There was no growth in the gold's CDS due to the in situ annealing step $\left(250^{\circ} \mathrm{C}\right)$. However, an exponential increase in the CDS was measured as $T_{A}$ increased, where after annealing at $800^{\circ} \mathrm{C}$, all samples had gold particles with a mean CDS of $\sim 31 \mathrm{~nm}$. Samples from substrate-1 (argon in situ) showed a faster growth of CDS after annealing at $600^{\circ} \mathrm{C}$, which might be indicative of faster dewetting. This reasoning is backed up by the evidence from the SEM micrographs in figure 7.2).

\subsection{Intensity}

The intensity of the (111) reflection of gold increased exponentially as the annealing temperature was increased, as seen in figure 7.14. This increase is indicative to film dewetting and is similar to the results reported by Muller et al and Mizsei et al [210, 220]. The intensity increase was previously attributed to the increase in particle height and texture particularly in the vicinity of holes $[220,214]$, and indeed it was found to correlate with the estimated growth of the $\mathrm{Au}$ (111) crystallite domain size as well as the measured increase in the gold films' height profiles as described in our AFM measurements. 


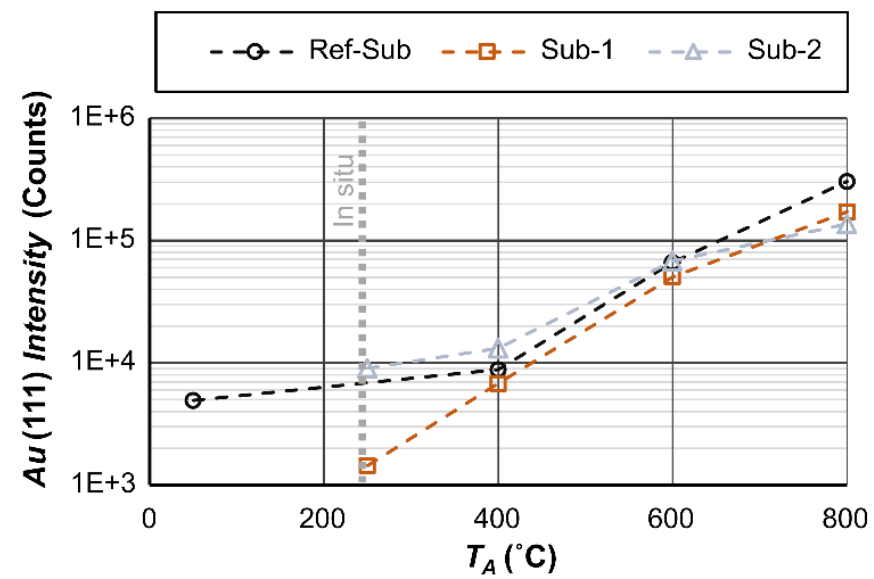

Figure 7.14: Measured Au (111) peak intensity for different samples as a function of the annealing temperature.

\subsubsection{Electrical Resistance Measurements}

Resistance measurements for each sample were carried out at a distance of $1 \mathrm{~cm}$ across the surface of the bare metal ring surrounding the $\mathrm{ZnO}$ film. These measurements are expected to show a sharp increase in resistivity as the conductive film disintegrates [221]. Such resistivity increase of conductive thinfilms due to annealing can be described by a percolation model [222]. One limitation for relying solely on resistive measurements to study film dewetting is the inability to measure any changes beyond the point when the films become discontinuous.

In order to evaluate the protective effect against metal dewetting due to having a $\mathrm{ZnO}$ overlayer, we measured the contact impedance as a function of the annealing temperature, for samples having $\mathrm{ZnO}$ deposited onto the metal layer, as well as samples having $\mathrm{ZnO}$ deposited directly on sapphire. In the latter case, although there was no $\mathrm{Cr} / \mathrm{Au} / \mathrm{Cr}$ layer under the $\mathrm{ZnO}$ film, but a thin metallic ring existed around the $\mathrm{ZnO}$ covered area to allow these measurements, as seen in figure 7.15. Since this contact area was suspected to 
agglomerate at temperatures lower than the metal areas covered by $\mathrm{ZnO}$, it was interesting to compare resistance variations for both types of samples used in this study: samples with a full metal contact under the $\mathrm{ZnO}$ layer, and samples with $\mathrm{ZnO}$ directly sputtered onto the sapphire substrate.
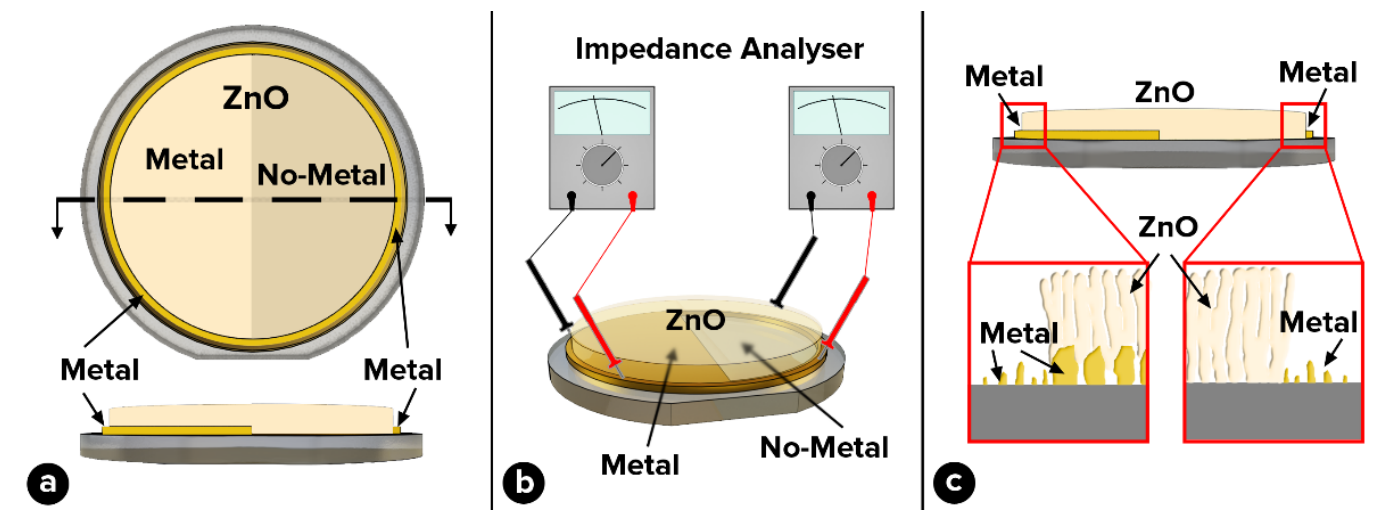

Figure 7.15: A sketch showing the impedance measurement setup on the different parts of the samples. a) Shows the top view of a given substrate and the corresponding cross-section; b) shows the full structure of the substrates, and the way the probes of the impedance analyser are fixed during measurement; c) the cross-section of a substrate showing both types of samples: $\mathrm{ZnO}$ sputtered directly on the substrate, and $\mathrm{ZnO}$ sputtered on top of the metal contact. This sketch also visualizes the morphological and dewetting related changes in a given sample after annealing.

The interest was in examining whether the encapsulated metal film would provide a path of lower electrical resistance during these measurements, as shown in Figure 7.16. 

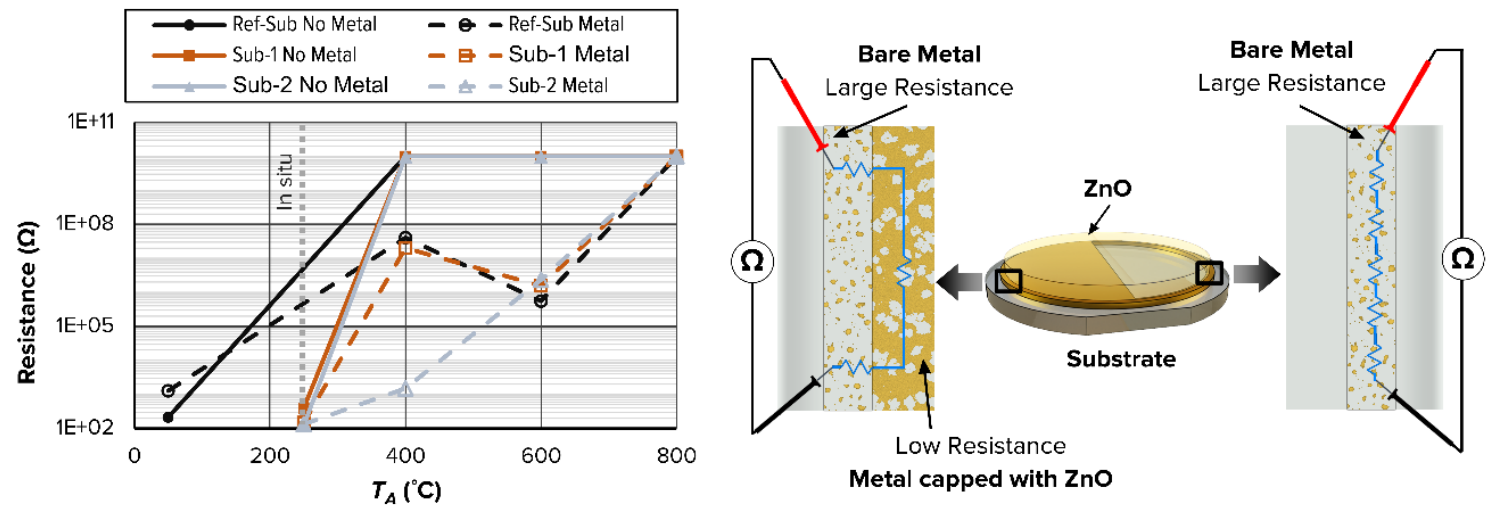

Figure 7.16: The measured surface resistance of our samples as a function of the annealing temperature. A sketch showing a perspective view and a top view of our sample structure, to highlight the metal contact geometry used for resistance measurements. The $\mathrm{ZnO}$ layer is drawn transparent for clarity. The left part shows a sample with a metal layer capped with $\mathrm{ZnO}$, while the right part shows a sample with no metal layer under the $\mathrm{ZnO}$ film.

The resistance measurements confirmed our latter speculation, where samples with $\mathrm{ZnO}$ directly grown onto sapphire had a resistance less than $1 \mathrm{k} \Omega$ for $T_{A} \leq$ $250^{\circ} \mathrm{C}$. Annealing at a higher temperature seemed to cause total metal layer agglomeration, as an open-circuit was measured. This condition was plotted at $10 \mathrm{G} \Omega$, which is equivalent to the input impedance of our analyser.

In contrast, samples with the encapsulated metal layer showed slower and gradual resistance increase as the annealing temperature was raised. At $T_{A}=400^{\circ} \mathrm{C}$, resistance was around $1 \mathrm{k} \Omega$ for the sample from substrate- 2 , while the other two samples had a resistance of $\approx 10 \mathrm{M} \Omega$. This difference in the measured resistance value for substrate- 2 supports the idea suggested by the high voltage SEM findings with regards to a slower dewetting progress for that sample, as the area fraction still covered by the metal film has been shown to vary in a linear relationship with the normalized surface resistance of the film 
[223]. As the annealing temperature was increased to $600^{\circ} \mathrm{C}$, the resistance for all three samples was $\approx 1 \mathrm{M} \Omega$. Such decrease in resistance value can be plausibly attributed to the metal film's grain growth, thereby minimizing electron scattering at grain boundaries [224]. This result also indicates that the $\mathrm{ZnO}$ encapsulation slows down the metal dewetting process, as an ohmic behaviour was still measured at such annealing temperature. Finally, an opencircuit was measured for samples that were annealed at $800^{\circ} \mathrm{C}$, indicating the total agglomeration of the encapsulated metal layer.

Thus, our resistance measurements indicate that total metal film agglomeration occurs when the annealing temperature is in excess of $250^{\circ} \mathrm{C}$. However, with the $\mathrm{ZnO}$ overlayer, this temperature threshold is pushed to $600^{\circ} \mathrm{C}$.

\subsection{Chapter Summary}

- Measurements confirmed the optimization of the $\mathrm{ZnO}$ films with annealing, where the average grain diameter increased from $\approx 50 \mathrm{~nm}$ for the as-sputtered films to $\approx 150 \mathrm{~nm}$ when annealed at $800^{\circ} \mathrm{C}$. The residual stress of the (002) textured $\mathrm{ZnO}$ films was eliminated by heating at temperatures $\geq 400^{\circ} \mathrm{C}$, and the $\mathrm{ZnO}(002)$ crystallite domain size increased from $8 \mathrm{~nm}$ as sputtered to $45 \mathrm{~nm}$ when heated at $800^{\circ} \mathrm{C}$.

- Such active layer structural optimization was accompanied by a gradual degradation of the $\mathrm{Cr} / \mathrm{Au} / \mathrm{Cr}$ contact structure underneath, due to dewetting. AFM, SEM, and EDS imaging indicated void initiation at $400^{\circ} \mathrm{C}$, after which void growth proceeded, and coalescence was 
dominant at $800^{\circ} \mathrm{C}$, resulting in $\mathrm{Au}-\mathrm{Cr}$ particles having an average diameter $\approx 1.5 \mu \mathrm{m}$.

- This metal layer agglomeration process was accelerated in areas where the $\mathrm{ZnO}$ overlayer was absent, leading to more dispersed and smaller particles with an average diameter of $\approx 500 \mathrm{~nm}$ at $800^{\circ} \mathrm{C}$.

- XRD measurements indicated an exponential increase in the Au (111) reflection intensity as the annealing temperature increased. The Au (111) crystallite domain size increased from $6 \mathrm{~nm}$-as deposited- to 31nm after annealing at $800^{\circ} \mathrm{C}$.

- Electrical measurements indicated that the resistance of the $\mathrm{Cr} / \mathrm{Au} / \mathrm{Cr}$ films increased from $\approx 100 \Omega$ for the as deposited films to $\approx 10 \mathrm{M} \Omega$ at 400 $600^{\circ} \mathrm{C}$ for films capped with a $\mathrm{ZnO}$ layer, to an open circuit at $800^{\circ} \mathrm{C}$. For areas that had no $\mathrm{ZnO}$ on top, resistance was found to be infinite for temperatures $\geq 400^{\circ} \mathrm{C}$.

Thus, we report severe agglomeration and dewetting of thin gold films occurring at temperatures well below the melting point. This process was found to be slower yet similar when the metal films were encapsulated with a thicker zinc oxide layer. Based on these findings, the highest recommended annealing temperature before agglomeration/functional failure of the metal contact layer was $400^{\circ} \mathrm{C}$. Given the importance of high temperature thermal annealing on the quality of the resulting $\mathrm{ZnO}$ films, and the incompatibility of the $\mathrm{Cr} / \mathrm{Au} / \mathrm{Cr}$ contacts with such a procedure, new contact materials with suitable properties are investigated in the next chapter. 


\section{CHAPTER 8}

\section{Results D: Conductive Zinc Oxide Contacts}

As concluded from the study presented in chapter 7 , gold metal contacts are found incompatible with the thermal annealing step, which is required for improving the structural and electrical properties of the active zinc oxide layer in our device structure. Thus, alternatives were sought, and it was decided to use n-type doped zinc oxide films for creating our contacts.

Both aluminium doped zinc oxide $(\mathrm{AZO})$ and gallium doped zinc oxide (GZO) options were considered, as these materials would possess the same crystal structure as our active zinc oxide layer, which would provide well matched ohmic contact materials [225, 226, 227] for our device purposes, and act as seed layers for growing c-axis oriented zinc oxide films. Another advantage for using n-type doped zinc oxide contacts is their optical transparency. This transparency supplements the secondary objective of this work, by allowing us to create fully transparent transducer structures on transparent c-axis oriented sapphire substrates. 


\subsection{Chapter Objectives}

This primary objective of the work presented in this chapter is:

- The deposition of electrically conductive doped zinc oxide contacts.

The secondary objectives of the presented work are:

- The deposition of doped $\mathrm{ZnO}$ contacts that are (002) oriented.

- The deposition of optically transparent doped $\mathrm{ZnO}$ contacts.

\subsection{TCO Fabrication}

In this part of the study, aluminium doped and gallium doped zinc oxide targets were used to sputter the contact layers. Both 3 inch targets were supplied from Kurt-Lesker, and had a 2\% Ga or $\mathrm{Al} / 98 \% \mathrm{ZnO}$ composition, with a $99.999 \%$ purity. RF magnetron sputtering using our HHV system was employed.

The first run for each target utilized the same sputtering conditions used in chapters 5 and 6 for creating the buffer $\mathrm{ZnO}$ layer; i.e., 150W RF power, $6 \mathrm{sccm}$ argon gas flow at $1.5 \mathrm{E}-2 \mathrm{mbar}, 66 \mathrm{~mm}$ substrate to target distance, with no substrate heating, and for a duration of 1 hour. This resulted in films having a thickness of $\approx 500-700 \mathrm{~nm}$. While the gallium doped film looked more transparent, both resulting films had a strong brown tint and were not electrically conductive.

Therefore, quick dry runs were employed to produce a recipe that allows the creation of uniform, transparent, and conductive doped zinc oxide films. As literature [228, 229, 226] indicated that better (002) selectivity and electrical 
conductivity were achieved when sputtering aluminium-doped $\mathrm{ZnO}$ films at lower pressures in the range of 1 - $5 \mu$ bar, a run was carried out using a pressure of $1 \mathrm{E}-3 \mathrm{mbar}$, which is one order of magnitude lower than the first sputtering attempt. The resulting gallium and aluminium doped samples were still tinted and nonconductive. In addition, both samples had four concentric circular regions with alternating transparency levels, as seen in figure 8.1.

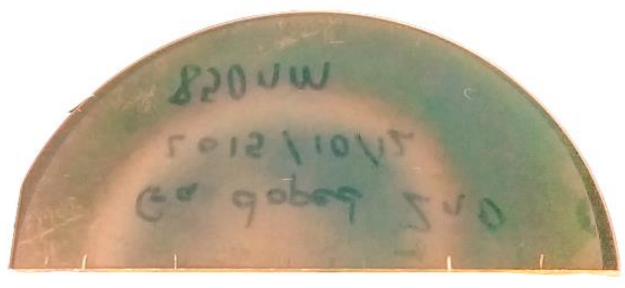

Sample Image

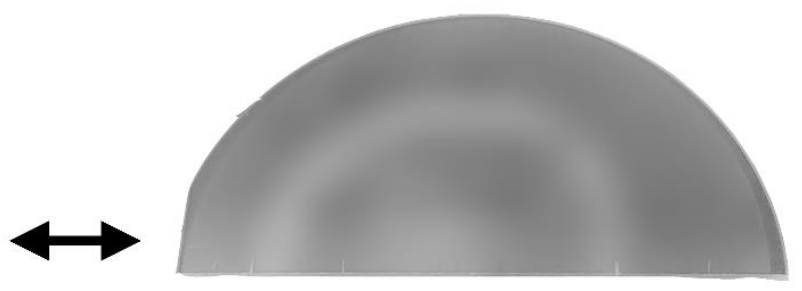

Grayscale Processed Image

Figure 8.1: Direct and processed (for clarity) sample images, showing the four different regions of alternating transparency.

In this figure, image processing was done to remove the chromatic tones and dull out speckles, markings, and scratches, while preserving the useful grayscale information. Lighter regions seen in these images are milky and translucent, whereas darker areas are highly transparent. Examination using optical microscopy revealed a flaky and damaged nature for the translucent parts of the films, especially in the first central region, while the films looked smooth and clear in the highly transparent regions. Thickness measurements using the profiler showed no thickness variations around the features, where 3 different locations were measured within each region. As these concentric regions are a recurrent theme in this chapter, they are denoted early on as regions 1, 2, 3, and 4; i.e., R1 - R4, starting from the centre outwards (figure 8.2). 
Despite being previously discouraged from heating the substrate during $\mathrm{ZnO}$ sputtering to avoid the undesired (100) and (101) crystal orientations (section 5.5.4), the next iteration was based on the reported suggestion that heating is required to produce electrically conductive doped $\mathrm{ZnO}$ films [227, 230, 191]. Thus, a gallium doped zinc oxide film was sputtered using the same low pressure of $1 \mathrm{E}-3 \mathrm{mbar}$, but at a substrate temperature of $200^{\circ} \mathrm{C}$. The resulting film looked generally more transparent; however, the four concentric regions seemed pushed outside, while the outer milky ring (region 3) became wider and less transparent. The whole sample was not electrically conductive, except for the small and transparent region 4 near the edge of the substrate. That region was the closest part of the substrate to the heat source, and was both transparent and conductive, with a resistivity of $\approx 4.8 \Omega$. $\mathrm{cm}$.
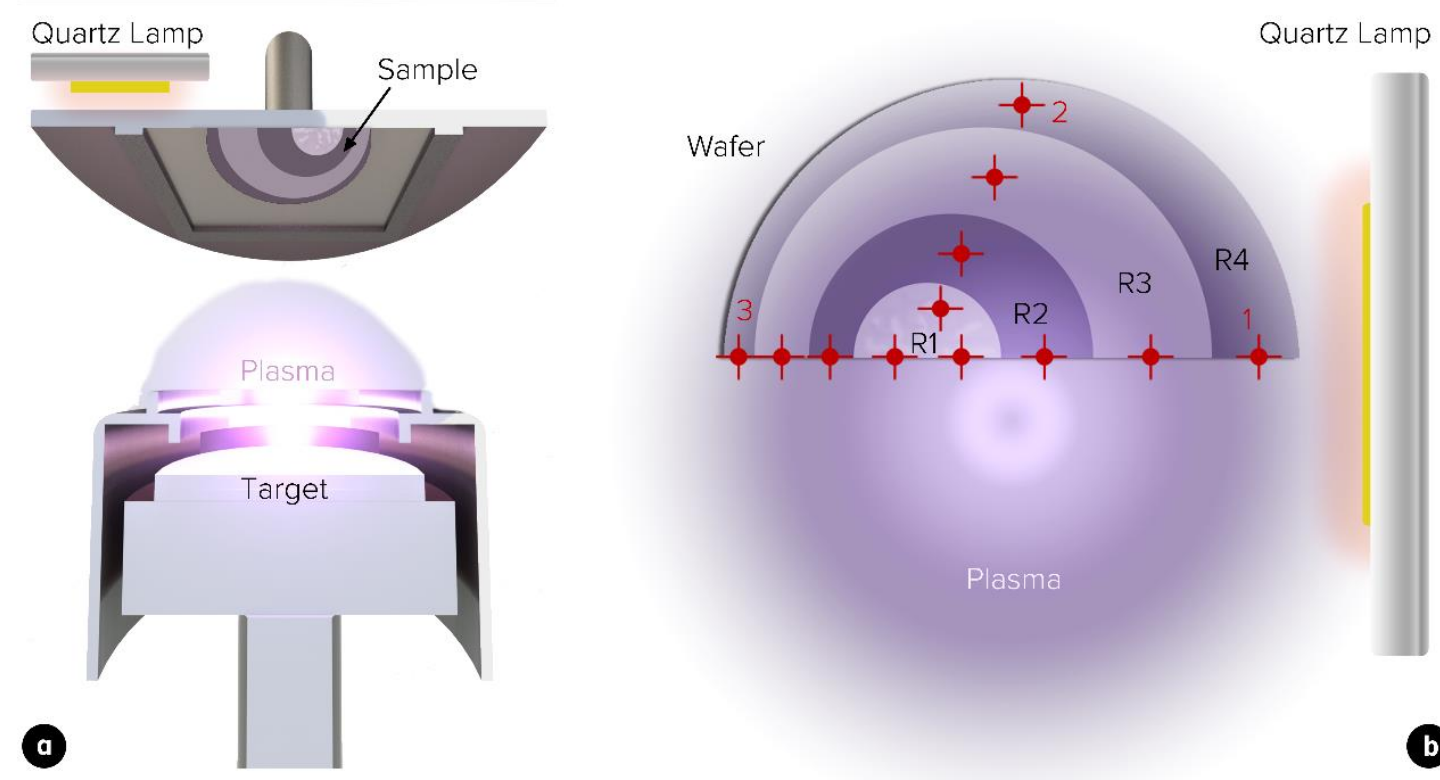

Figure 8.2: Schematics showing the sample placement inside the sputter coater, and the different regions (R1-R4) of each film. The dots represent the places at which thickness and resistivity measurements were carried out. (a) side view, (b) plan view 
Given its favourable optical and electrical properties, the next goal was to maximize the area of region 4, while reducing the footprint of the damaged regions.

Literature indicated that the observed film damage can be attributed to bombardment by energetic oxygen ions during doped and undoped $\mathrm{ZnO}$ sputtering [205, 191, 231]. In these reports, film damage is observed at substrate positions facing the racetrack eroded target area, and is caused by the $\mathrm{O}^{-}$ions accelerating from the target towards the substrate. However, to our knowledge, there is no previous work reporting the observed concentric damaged regions as found in our study. This damage pattern is assumed to be due to the lateral distribution of the different species of negative ions $\left(\mathrm{O}^{-}\right.$and $\mathrm{O}_{2}^{-}$in particular $)$ within the RF magnetron sputtering plasma [232], where these ions are mostly generated near the target centre and the eroded racetrack area.

The next step was to sputter while placing the substrate further from the target to minimize the radiation damage, and raise the substrate temperature to possibly increase the area of region 4 . Thus, both gallium and aluminium doped zinc oxide films were sputtered at a substrate-target distance of $71 \mathrm{~mm}$ and a temperature of $250^{\circ} \mathrm{C}$. The resulting films were comparable, with the aluminium doped zinc oxide sample appearing slightly more transparent. All regions seemed to be pushed towards the centre, as if the plasma profile became tighter. This meant that the conductive and transparent region 4 became bigger. The damaged regions 1 and 3 had a resistivity of $\approx 5 \mathrm{k} \Omega . \mathrm{cm}$ and $1.8 \mathrm{k} \Omega . \mathrm{cm}$, respectively; while the undamaged regions 2 and 4 had a resistivity of $115 \Omega . \mathrm{cm}$ and $4 \Omega . \mathrm{cm}$, respectively. Thus, it became evident that the sputtering plasma profile could be effectively tweaked to minimize the film damage due to bombardment by negative ions. This would be achieved 
through controlling the substrate temperature and the substrate-target distance and placement.

It should be noted that at this stage, substrate heating was applied using a single quartz lamp, fixed at one side on top of the holder. As temperature increased, the centre of the observed film pattern seemed to be pushed away from the heat source. This meant that region 4 was larger in the vicinity of the heat source. Thus, to increase the area of this conductive and transparent region, the sample holder was modified, to allow the heater to sit directly on top of the whole substrate. This favourable change had a significant impact on increasing the attainable heating rate and final temperature, while permitting the quartz lamp to act as an isotropic heat source for the whole substrate.

The next parts of this doped zinc oxide growth experiment involved varying the plasma's RF power between $150 \mathrm{~W}$ and $250 \mathrm{~W}$, which directly affects the voltage and energy of the bombarding ions. In addition, the target-tosample distance was varied between $55 \mathrm{~mm}$ and $92 \mathrm{~mm}$, and the substrate temperature was varied between $250^{\circ} \mathrm{C}$ and $325^{\circ} \mathrm{C}$. Finally, sputtering off the plasma-axis was attempted using various conditions to avoid having the eroded film area. The main processing iterations to improve the film qualities are highlighted in figure 8.3 .

The optimal conditions obtained through these iterations allowed us to consistently deposit electrically conductive and visibly transparent doped zinc oxide films with a good $\mathrm{ZnO}$ (002) selectivity, and with no noticeable damage. The final resistivity of these films was in the range of $0.3 \Omega . \mathrm{cm}$, where optical transmission was as high as $75 \%$ down to the near UV range, while the $\mathrm{ZnO}$ (002):(100) selectivity was larger than 200. The general results of these sputtering iterations are presented and discussed in the following sections of this chapter. 


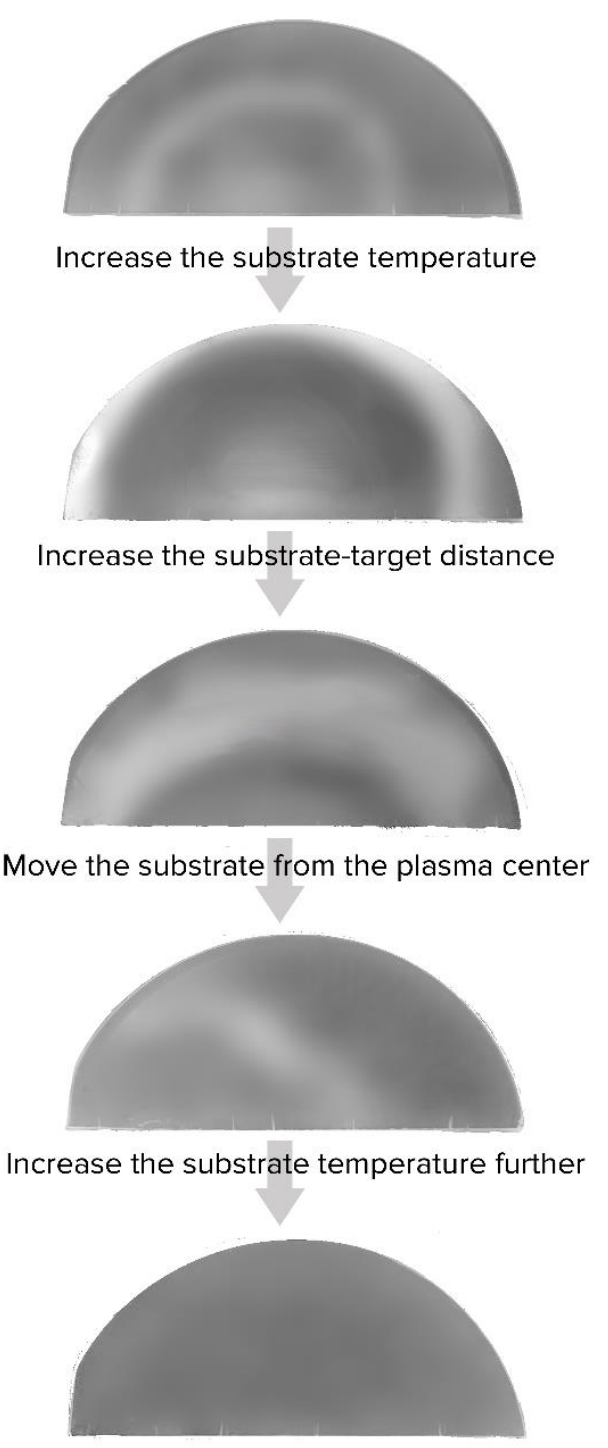

Figure 8.3: Photographs showing the different regions of each sample, as processing was adjusted to create conductive and transparent doped zinc oxide films. The photographs have been digitally processed to remove the small-scale features and enhance contrast between the different regions.

\subsection{Film characterization}

X-ray diffraction, scanning electron microscopy, optical absorbance and transmission, and electrical resistivity measurements were conducted for all 
samples included in this study. Over the course of the following sections, the main relevant processing parameters are isolated, in order to evaluate their effects on the final film qualities.

\subsubsection{XRD}

Having the doped $\mathrm{ZnO}$ bottom contact grown along the c-axis direction is very important to the next steps of our device fabrication, given that this layer would act as a well-matched substrate material to the piezoelectric $\mathrm{ZnO}$ film.

XRD measurements indicated that regions 1,2 , and 3 were generally comparable in their properties, while the outer region (4), furthest away from the plasma centre, had the largest crystallite domain size, $\mathrm{ZnO}$ (002) peak intensity, and $\mathrm{ZnO}(002)$ selectivity.

\subsubsection{Crystallite domain size}

For instance, using different sputtering RF powers at $200^{\circ} \mathrm{C}$ led to a similar mean crystallite domain size in regions 1-3, which was about $8 \mathrm{~nm}$, while the fourth region was found to have a larger mean domain size of up to $35 \mathrm{~nm}$, corresponding to increased sputtering power, as seen in figure 8.4. 


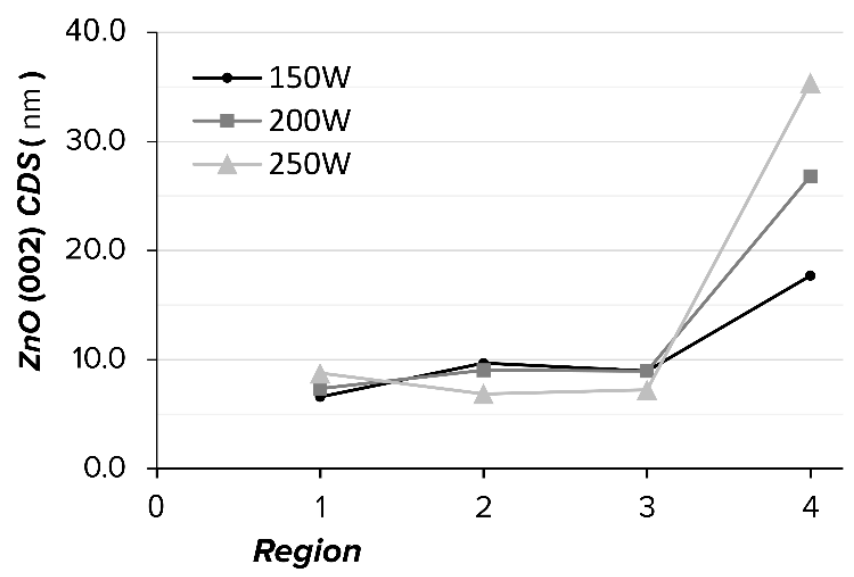

Figure 8.4: The average $\mathrm{ZnO}$ (002) crystallite domain size in each region of the aluminium doped $\mathrm{ZnO}$ samples, as a function of the sputtering RF power.

The mean crystallite domain size in this region was estimated at $18 \mathrm{~nm}, 26 \mathrm{~nm}$, and $35 \mathrm{~nm}$ for the RF powers of $150 \mathrm{~W}, 200 \mathrm{~W}$, and 250W, respectively.

\subsubsection{ZnO (002) peak intensity}

In a similar fashion, increasing the sputtering RF power seemed to have little to no effect on the measured $\mathrm{ZnO}$ (002) peak intensities of regions 1-3, while it was found to directly correlate with the (002) peak intensity of region 4 , as seen in figure 8.5. This increase was as large as one order of magnitude per 50W increase, confirming that the deposited doped $\mathrm{ZnO}$ films have relatively large (002) crystallites [201] in region 4, over a wide range of the sputtering RF power. 


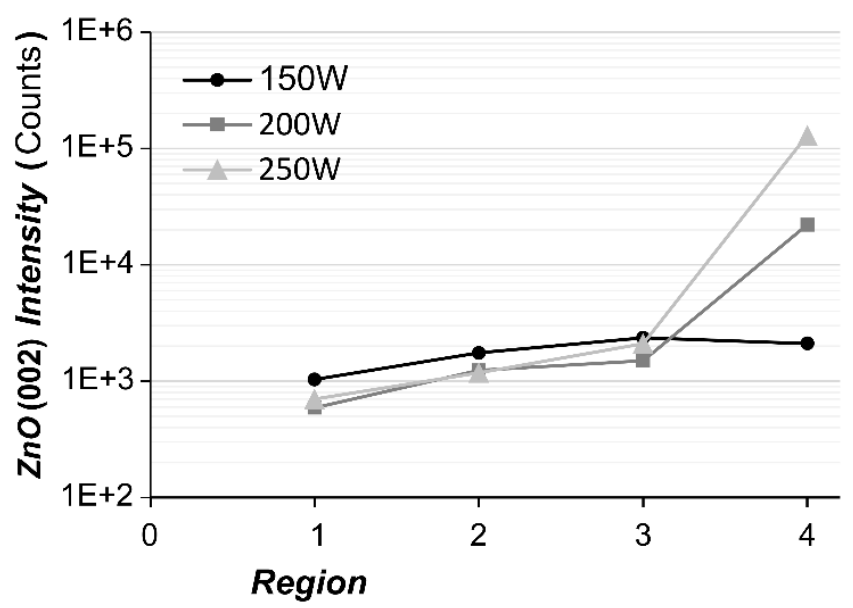

Figure 8.5: The measured $\mathrm{ZnO}(002)$ peak intensity in each region of the aluminium doped $\mathrm{ZnO}$ samples as a function of the sputtering RF power.

\subsubsection{3 $\mathrm{ZnO}(002)$ selectivity}

With regards to the crystal orientation selectivity, RF magnetron sputtering using the aforementioned parameters was found to generally favour the $\mathrm{ZnO}$ (002) phase. Therefore, the doped zinc oxide films grew along the desired caxis. However, $\mathrm{x}$-ray diffraction measurements indicated that oxygen radiation damage was detrimental to this orientation selectivity, where region 4 was found to be significantly more (002) oriented in comparison to other regions, as seen in figure 8.6.

Increasing the RF power from $150 \mathrm{~W}$ to $250 \mathrm{~W}$ had insignificant effect on the $\mathrm{ZnO}$ orientation selectivity in regions 1 through 3, where a slight but consistent increase in the (002):(100) ratio was observed, along the radial direction, from about 6 to 10 .

However, the (002) selectivity in region 4 was found to increase with increasing the sputtering RF power, where it was around 20 at $150 \mathrm{~W}$, and increased to approximately 170 at $250 \mathrm{~W}$. 
These results are resonant with other literature reports [205, 233, 234, 229], which suggest that orientations with lower free-surface-energy are more prone to damage due to ion bombardment. In this case, the $\mathrm{ZnO}^{\prime} \mathrm{s}$ (002) orientation, being the most closely packed with the least free-surface-energy is easily attacked by the negative energetic ions during deposition. This preferential damage causes the less packed (100) and (101) orientations to become more abundant in the affected regions.

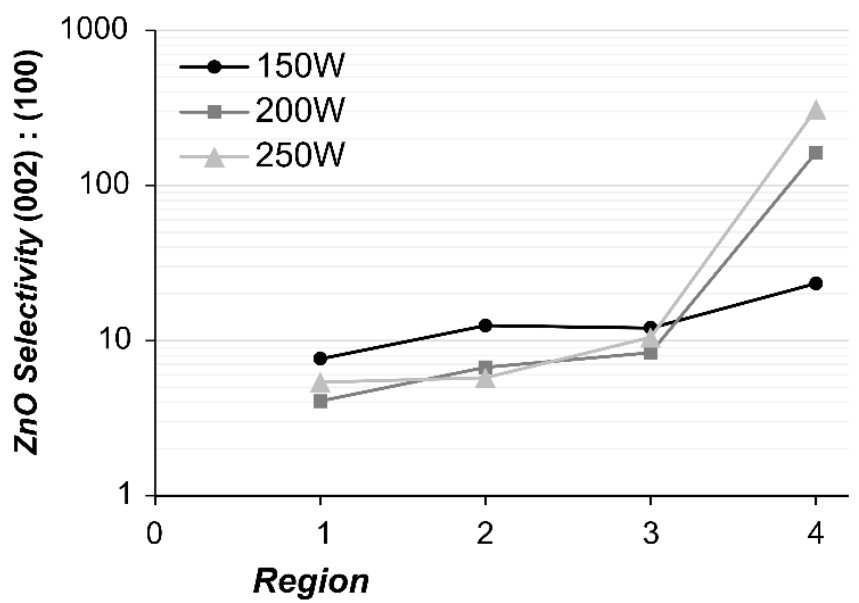

Figure 8.6: The estimated $\mathrm{ZnO}$ (002):(100) selectivity in each region of the aluminium doped $\mathrm{ZnO}$ samples, as a function of the sputtering RF power.

This significant (002) selectivity boost in region 4 due to the increased RF power can also be attributed to the increased sputtering voltage, which is expected to result in longer mean-free-paths for the different plasma species, provided that other sputtering conditions remain fixed. Thus, assuming that most negative oxygen ions are generated near the centre of the target (region 1) and the eroded racetrack area (region 3), and given a constant target to sample distance, having a longer mean-free-path and a stronger accelerating field are expected to 
limit the plasma species lateral movement. Consequently, this reduces the spread of radiation damage to the other regions, particularly to region 4 .

When comparing the major undesired $\mathrm{ZnO}$ crystal orientations found in our films; i.e., the (100) and (101) orientations, the latter was found to generally have a higher peak intensity, possibly due to it being less geometrically packed, and thus less susceptible to oxygen radiation damage during sputtering.

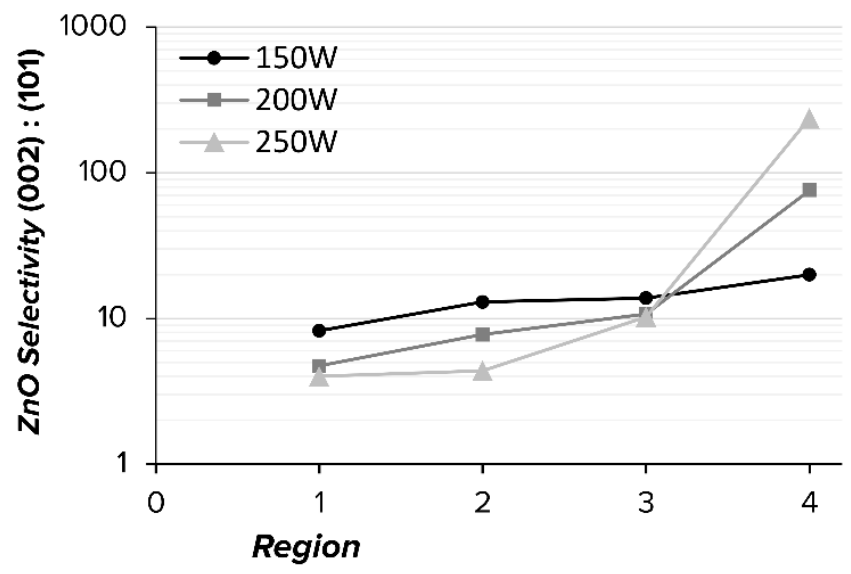

Figure 8.7: The estimated $\mathrm{ZnO}$ (002):(101) selectivity in each region of the aluminium doped $\mathrm{ZnO}$ samples, as a function of the sputtering RF power.

As the optimization of the processing parameters progressed, better $\mathrm{ZnO}(002)$ selectivity and higher (002) peak intensity were observed, particularly for the least damaged area; i.e., region 4. In addition, it was noted that radiation damage seemed negatively correlated with selectivity. Thus, increasing the area of the undamaged regions by moving the sample away from the plasma centre (off-axis sputtering) was crucial, to ensure having large film areas with highly (002) oriented doped zinc oxide. 


\subsubsection{SEM}

Morphological examination using the secondary electron detector of the scanning electron microscope revealed that the $\mathrm{ZnO}$ film grains became larger when moving outwards from the centre of the observed damage pattern, as shown in figure 8.8. The sample in this figure is the same aluminium doped sample sputtered at 200W in the previous XRD section.

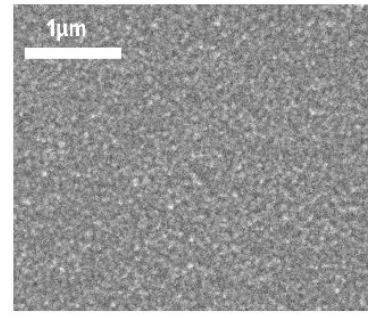

R1

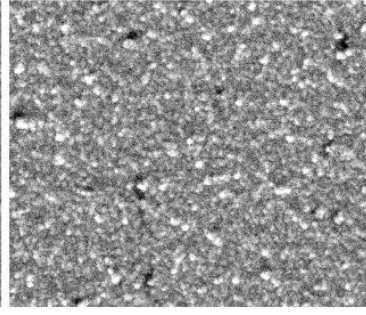

R2

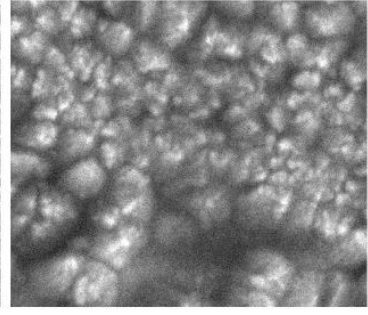

R3

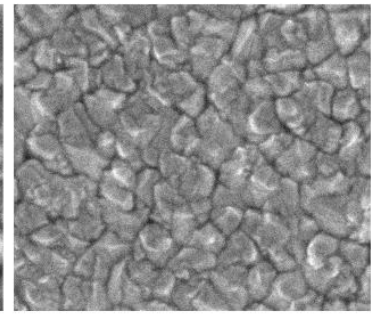

R4

Figure 8.8: SEM images of the different regions within an aluminium doped sample.

Thus, region 1 had the smallest grain diameter, which was estimated at $\varphi_{\operatorname{gr}(\mathrm{Zno})} \sim 35 \mathrm{~nm}$, while region 2 had slightly larger grains of about $50 \mathrm{~nm}$. Region 3 showed clear signs of surface damage, where large variations in the grain sizes and shapes were observed. These grains had diameters ranging from $50 \mathrm{~nm}$ to $200 \mathrm{~nm}$. Finally, grains in region 4 appeared faceted, indicating a highly-ordered crystal nature, and had an average diameter of $\sim 260 \mathrm{~nm}$.

These images confirmed the previous XRD results, which showed that the crystallite domain size and the $\mathrm{ZnO}(002)$ peak intensity grew in a gradual manner when moving from regions 1 to 3 , while region 4 showed significantly larger values. 


\subsubsection{UV/VIS Spectroscopy}

Without the use of off-centre sputtering, transparency was visibly non-uniform across a given sample, due to damage by energetic oxygen ions. As mentioned previously, the samples were damaged and translucent near the plasma centre, and the area directly on top of the racetrack damage on the doped $\mathrm{ZnO}$ target; i.e., regions 1 and 3, respectively.

Optical transmission measurements for a typical sample are shown in figure 8.9. These measurements, for a gallium doped sample in this instance, reflect a clear disparity between the more transparent and less transparent regions.
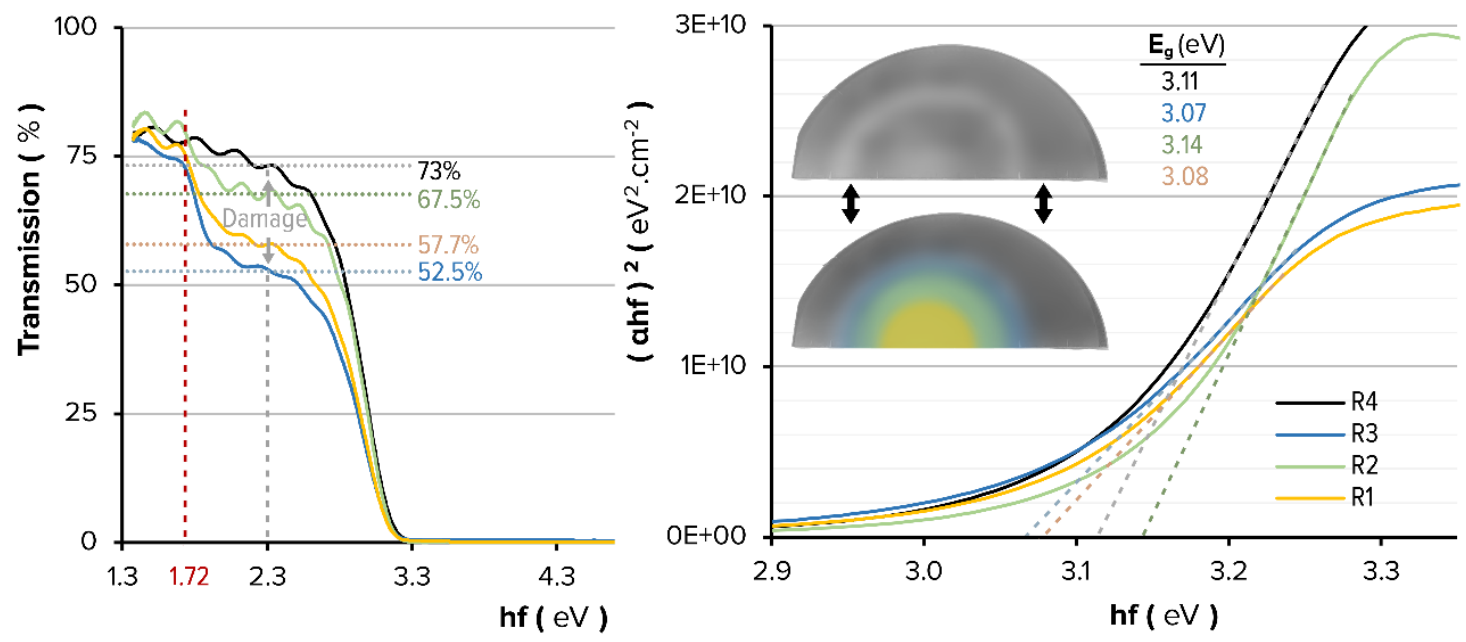

Figure 8.9: The measured optical transmission level (left) and estimated bandgap (right) in each region of a typical doped $\mathrm{ZnO}$ sample. This sample is shown in the inset, where the same image was colorized to reflect the different regions. Here yellow, green, blue, and black correspond to regions 1, 2, 3, and 4 , respectively. 
The highly transparent regions 2 and 4 show a slight decrease in transmission for incident photons with energies below the bandgap, from about $80 \%$ at $1.3 \mathrm{eV}$ to $68 \%$ at $3 \mathrm{eV}$. On the other hand, the radiation damaged regions 1 and 3 show a sudden drop in photon transmission percentage at $1.72 \mathrm{eV}$, which corresponds to a photon wavelength of $720 \mathrm{~nm}$. This transmission drop was about 20 percent for each region, and was found persistent until the bandgap edge.

Estimating the film material's bandgap at each region using Tauc plots indicated slight bandgap variations. Regions 2 and 4 had higher bandgaps of 3.14 and 3.11, respectively, while the damaged regions 1 and 3 had a bandgap of 3.08 and 3.07 respectively.

\subsubsection{Gallium versus aluminium doping}

As sputtering parameters changed, gallium and aluminium doped $\mathrm{ZnO}$ films were compared. UV/Vis measurements indicated that given identical sputtering conditions, gallium doped $\mathrm{ZnO}$ possessed a lower bandgap than aluminium doped $\mathrm{ZnO}$. This bandgap difference was about $0.05-0.15 \mathrm{eV}$ in all four regions of the compared samples. 


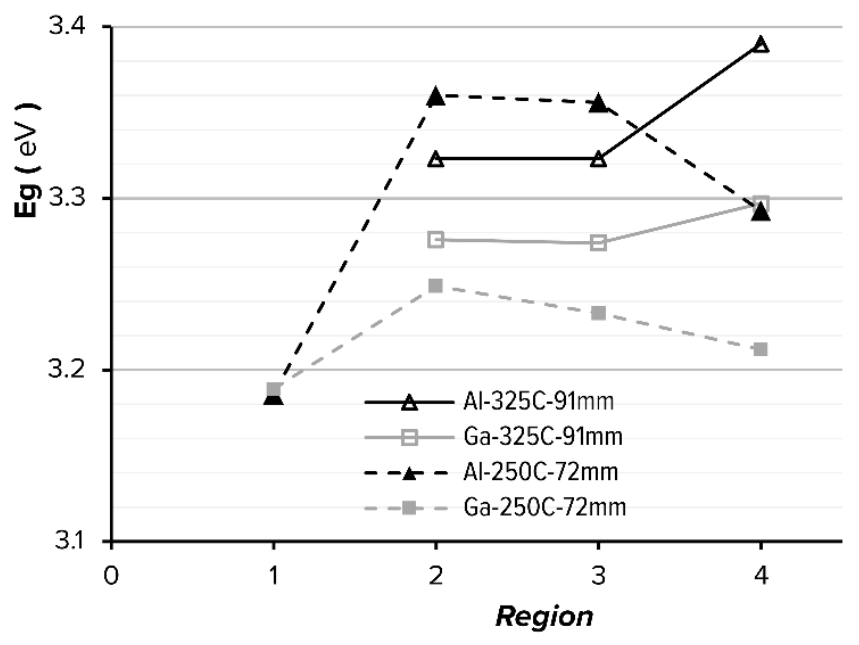

Figure 8.10: The estimated bandgap in each region of two gallium doped and two aluminium doped $\mathrm{ZnO}$ samples.

In the plots shown in figure 8.10, the two compared batches of samples differed by the substrate temperature and substrate to target distance. The first batch was processed at $325^{\circ} \mathrm{C}$ and at a distance of $91 \mathrm{~mm}$, while the other batch was processed at $250^{\circ} \mathrm{C}$ and a distance of $72 \mathrm{~mm}$. Increasing the sputtering temperature and the substrate to target distance seems to bring the gallium and aluminium doped samples' bandgaps closer to $3.3 \mathrm{eV}$.

\subsubsection{The effects of RF power}

When keeping the rest of the sputtering parameters identical, optical measurements indicated that damage near the plasma centre became more wide-spread as the sputtering RF power increased, as shown in figure 8.11. 

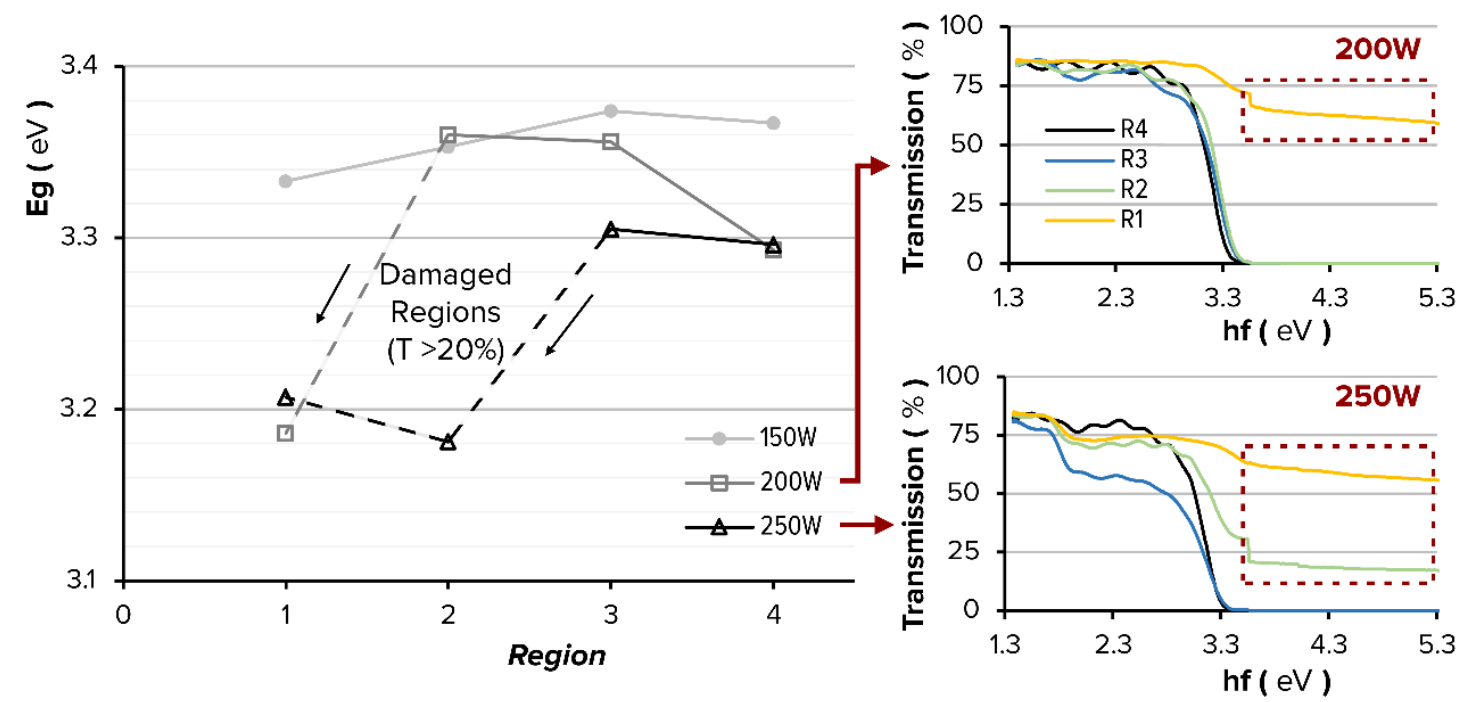

Figure 8.11: The estimated optical bandgap in each region of three aluminium doped $\mathrm{ZnO}$ samples, sputtered at 150W, 200W, and 250W. For each sample with weakly absorbing damaged regions (transmission $>20 \%$ ), the measured transmission is shown to the right side. The red dotted box highlights transmission through the damaged regions. Here yellow, green, blue, and black correspond to regions 1, 2, 3, and 4 , respectively.

This damage was manifested in not achieving complete absorption of photons with energies that exceed the material's bandgap. Thus, as the sputtering RF power increased from $150 \mathrm{~W}$ to $200 \mathrm{~W}$, the film in region 1 became unable to absorb most of the incident photons with energies higher than the bandgap indicated by the absorption edge at $3.18 \mathrm{eV}$. Similarly, when the sputtering RF power increased to $250 \mathrm{~W}$, both regions 1 and 2 became affected, and non-zero transmission higher than $20 \%$ was observed for photons with energies higher than the observed bandgap.

It should be noted that the three samples included in this section were aluminium doped, and these samples displayed a slight decrease in the estimated bandgap as the sputtering RF power increased. 


\subsubsection{The effects of substrate temperature}

Controlling the substrate temperature during sputtering of the doped $\mathrm{ZnO}$ films was found critical for defining the resulting films' optical properties. As the substrate temperature increased, the measured bandgap was found to increase in all regions, as seen in figure 8.12.

Thus, the bandgap increased from about $3.1 \mathrm{eV}$ at $30^{\circ} \mathrm{C}$ to $3.28 \mathrm{eV}$ at $325^{\circ} \mathrm{C}$. In addition, bandgap differences across the different regions of a given sample were found to decrease as temperature increased.

It is noted that the samples included in this study were gallium doped, with the $325^{\circ} \mathrm{C}$ sample being sputtered off-axis to avoid plasma damage near the centre; therefore, the point indicating region 1 is not present.

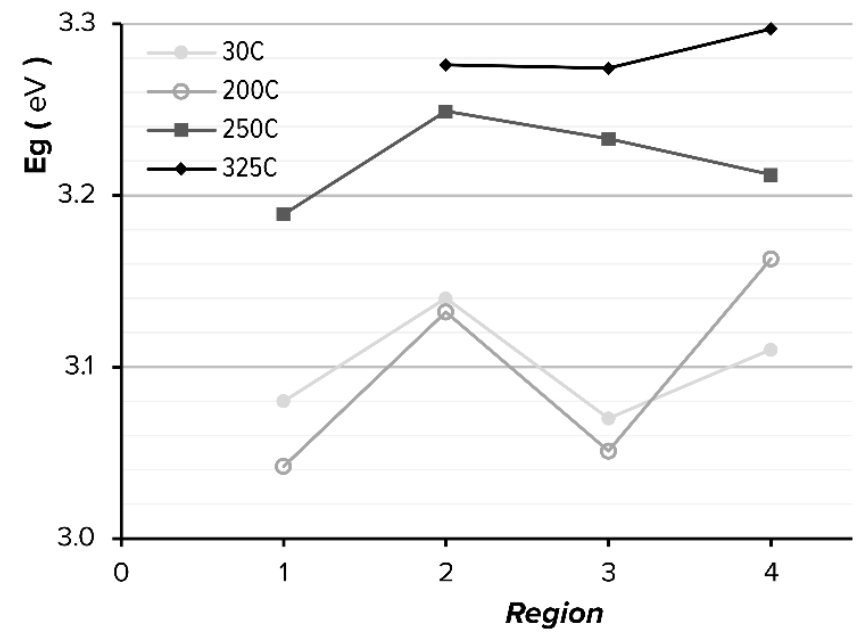

Figure 8.12: The estimated optical bandgap in each region of four gallium doped $\mathrm{ZnO}$ samples, sputtered at the temperatures of $30^{\circ} \mathrm{C}, 200^{\circ} \mathrm{C}, 250^{\circ} \mathrm{C}$, and $325^{\circ} \mathrm{C}$.

In summary, the size and location of the damaged circular pattern within the sputtered doped zinc oxide films was chiefly affected by the sputtering RF power, sputtering substrate temperature, sputtering pressure, as well as the 
sample to target distance. Having a higher sputtering power resulted in more severe damage, which was manifested by the lack of complete optical absorbance beyond the films' bandgap in the central regions. This damage increase can be attributed to the higher accelerating fields available at higher plasma powers. This eminently results in faster and more energetic negative ions bombarding the sample's surface during sputtering [232]. Accordingly, a maximum plasma RF power of 200W was adopted during the sputtering of our doped zinc oxide contacts. In contrast, using a higher sputtering pressure up to $\approx 10^{-2}$ mbar seemed to reduce the damage severity, probably due to the associated shorter mean-free-path of the plasma species. A similar result was reported by Hada et al. [229]. However, this approach was not a viable process remedy as it resulted in films having poor (002) selectivity. On the other hand, increasing the substrate temperature seemed to push the centre of the damage away from the heating source. For the relevant samples, this led to a larger region 4 at the side closer to the quartz lamp. Increasing the sample to target distance reasonably led to larger yet visibly less damaged and more transparent regions 1 and 3 . This is probably due to the longer vertical distance that the damaging negative ions would have to travel, which allows for more collisions to occur. Therefore, a large sample to target distance (maximum of $92 \mathrm{~mm}$ ) was favourable to create our transparent device contacts.

\subsubsection{Resistivity}

Resistivity measurements conducted in this chapter indicated that the doped zinc oxide films had significant variations in the measured electrical conductivity when moving across the different regions of a single sample. Resistivity was found to decrease by several orders of magnitude when moving from region 1 to region 4 , up to almost 7 orders of magnitude in some cases. It 
should be noted that the thickness variation between regions 1 and 4 was less than $20 \%$.

\subsubsection{The effects of RF power}

When considering the effect of the sputtering RF power, figure 8.13 shows that samples deposited at 150W, 200W, and 250W had similar resistivities of $\approx$ $4 \times 10^{6} \Omega . \mathrm{cm}$ and $\approx 3 \times 10^{3} \Omega . \mathrm{cm}$ in regions 1 and 2 , respectively. However, the more-conductive regions 3 and 4 had lower resistivity values when using higher RF powers, with the 150W sample showing a significantly high resistivity of $\approx 2 \times 10^{6} \Omega$.cm in the radiation damaged region 3 . Region 4 was highly conductive for all samples, where resistivity was measured to be smaller than $5 \Omega . c m$.

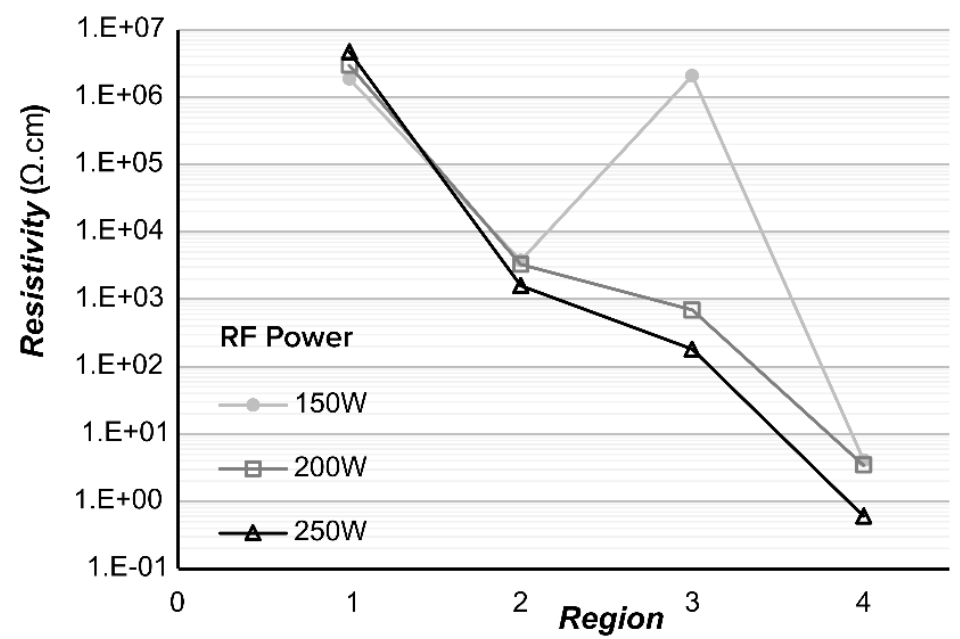

Figure 8.13: The measured resistivity in each region of three aluminium doped $\mathrm{ZnO}$ samples, sputtered at 150W, 200W, and 250W.

Samples included in this study were all aluminium doped and sputtered under similar conditions at $250^{\circ} \mathrm{C}$, except for the sputtering RF power. 


\subsubsection{The effects of substrate temperature}

A second resistivity study was aimed at isolating and understanding the effects of the substrate temperature on the achieved films' resistivity. Four different gallium doped zinc oxide films were sputtered using identical conditions, excluding the substrate temperature, which was varied between $30^{\circ} \mathrm{C}$ and $325^{\circ} \mathrm{C}$. Results indicated that using higher temperatures lead to significantly more conductive films, as shown in figure 8.14.

Similar to the previous study that examined the effects of the sputtering RF power, it was found that resistivity decreased when moving from region 1 to region 4. Interestingly, the highly-damaged region 1 had almost the same resistivity of $\approx 3 \times 10^{3} \Omega$.cm for all samples, except for the $325^{\circ} \mathrm{C}$ sample, which was deposited off-axis to avoid having this region altogether.

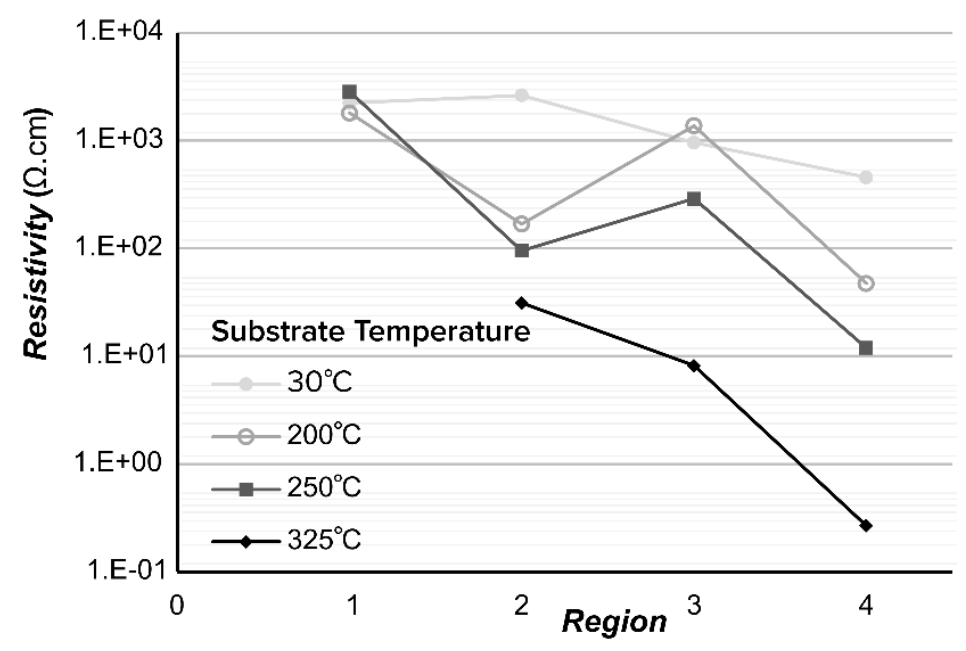

Figure 8.14: The measured resistivity in each region of four gallium doped $\mathrm{ZnO}$ samples, sputtered at the temperatures of $30^{\circ} \mathrm{C}, 200^{\circ} \mathrm{C}, 250^{\circ} \mathrm{C}$, and $325^{\circ} \mathrm{C}$.

Region 4 was the most conductive for all samples, with a resistivity dropping from $\approx 6 \times 10^{2} \Omega . \mathrm{cm}$ at $30^{\circ} \mathrm{C}$ to $\approx 0.3 \Omega$.cm for the sample sputtered at $325^{\circ} \mathrm{C}$. 
By examining the results of the previous two resistivity studies; i.e., the effects of the sputtering RF power and the effects of substrate temperature, it can be seen that the range of variation of resistivity in any aluminium doped sample is larger than that attainable in gallium doped $\mathrm{ZnO}$. Thus, aluminium doped $\mathrm{ZnO}$ showed a resistivity variation of 6-7 orders of magnitude between regions 1 and 4, while gallium doped samples typically showed a difference of 3 orders of magnitude. Since the measured bandgaps for both types of films differed only slightly, as indicated in the section 8.3.3.1, it can be deduced that the doping profile is more uniform across the surface of gallium doped zinc oxide films.

As highly conductive doped $\mathrm{ZnO}$ was desired, it was evident that sputtering should maximize the area of region 4 while avoiding the other regions. Sputtering off the plasma axis provided such a solution, along with increasing the substrate temperature and the substrate to target distance.

\subsection{Chapter Summary}

- Sputtering doped $\mathrm{ZnO}$ films with similar conditions used for pure $\mathrm{ZnO}$ sputtering led to surface damage. This damage is attributed to the film's bombardment by energetic oxygen ions during sputtering. The damage caused four concentric circular regions with alternating transparency to exist on every sample. Those regions were denoted as R1 to R4, starting from the centre of a given sample, then moving outwards.

- The outermost region R4 had the most favourable properties in regards to our defined objectives. For a given sample, R4 had generally good $\mathrm{ZnO}$ (002) orientation, the highest optical transmission, and the lowest electrical resistance. This high (002) selectivity in R4 is attributed to the 
lower severity of oxygen ion bombardment, as bombardment damage affects crystal faces with the least free-surface-energy.

- Increasing the substrate temperature during sputtering was favourable for maximizing the area of the outermost region $\mathrm{R} 4$, and decreasing the resistivity of that region.

- Increasing the substrate to target distance resulted in less damaged films, with a larger R4 region.

- Increasing the sputtering RF power resulted in an increased (002) selectivity and a lower resistivity in R4; however, it resulted in worse optical transmission properties, particularly in R1 and R2.

- The damaged regions R1 and R3 were less transparent than the undamaged regions R2 and R4.

- Moving the substrate away from the target centre was an effective method for reducing the film's surface damage, and increasing the area of R4.

- Given identical processes, gallium doped $\mathrm{ZnO}$ possesses a lower bandgap than aluminium doped $\mathrm{ZnO}$.

- By increasing the substrate temperature to $325^{\circ} \mathrm{C}$ during sputtering, while setting the substrate at a distance of $92 \mathrm{~mm}$ away from the target resulted in conductive doped $\mathrm{ZnO}$ films with a resistivity of $\sim 0.3 \Omega . \mathrm{cm}$. These films did not show any noticeable damage, and had an optical transmission in excess of $75 \%$, down to the near UV range. The $\mathrm{ZnO}$ (002) selectivity of these contacts was larger than 200. 
Given the favourable properties of the developed conductive $\mathrm{ZnO}$ contacts, the next step was to attempt the creation of all-ZnO transducer structures. 


\section{CHAPTER 9}

\section{Device Thickness Films}

\subsection{Chapter Objectives}

The main objective of the studies presented in this chapter is the creation of full device structures, as proposed in our design. These structures should have ohmic metal contacts, with a $3-10 \mu$ m thick $\mathrm{ZnO}$ layer in between. This $\mathrm{ZnO}$ layer should have the suitable electrical and structural properties to be fitting as a transducer for high frequency piezoelectric applications.

Thus, the main objectives of this chapter are:

- Primarily test the validity of the developed fabrication processes for delivering the required device structure.

- If the primary objective is met, then a secondary objective would be the development of an all-ZnO transducer structure, which opens the potential for creating a transparent acoustic and optic lens design. 


\subsection{First Structure Attempt}

At an earlier stage of this project, a full device structure was attempted based on the results described in chapters 5 and 6. This device structure had gold based bottom and top contacts, and was meant as a test experiment to evaluate the fabrication recipe limits, and to check for any unforeseeable problems. This fabrication process followed the optimal recipe suggested in chapter 5, and required a total of 12 hours to complete. For comparison purposes, both sapphire and borosilicate substrates were used.

Wafers were diced and cleaned before the bottom $\mathrm{Cr}(1 \mathrm{~nm}) / \mathrm{Au}(20 \mathrm{~nm}) /$ $\mathrm{Cr}(1 \mathrm{~nm})$ contact layer was deposited. Thereafter, a buffer $\mathrm{ZnO}$ layer was sputtered over a period of 1-hour at an RF power of $150 \mathrm{~W}$, and using a $6 \mathrm{sccm}$ argon gas flow-rate with a corresponding sputtering pressure of $1.5 \times$ $10^{-2}$ mbar. Next, an in situ annealing step at $250^{\circ} \mathrm{C}$ for 1 -hour in a $100 \%$ oxygen gas background $(6 \mathrm{sccm})$ was carried out. Subsequently, the substrate was allowed to cool down to room temperature, before sputtering the rest of the $\mathrm{ZnO}$ film during 8 hours. This long sputtering session used similar conditions to the buffer layer step, except for having a higher RF power of $240 \mathrm{~W}$. Finally, a $\mathrm{Cr}(1 \mathrm{~nm}) / \mathrm{Au}(20 \mathrm{~nm})$ top contact was deposited, to complete the device structure.

The resulting samples were visibly transparent, as seen in figure 9.1, and did not show signs of peeling when inspected using a 50X confocal microscope. 


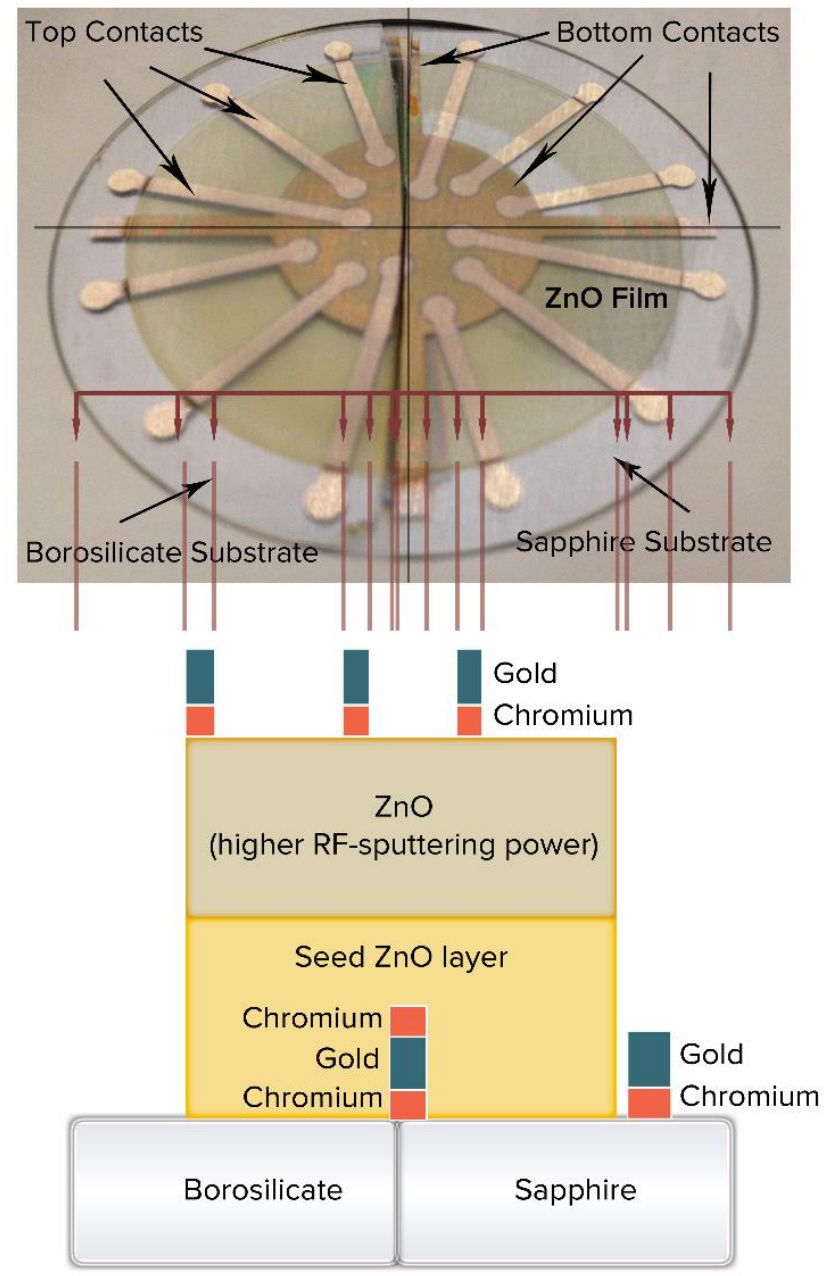

Figure 9.1: The $5 \mu \mathrm{m}$ thick $\mathrm{ZnO}$ films were sputtered on amorphous borosilicate glass (left) and c-axis oriented sapphire (right).

In this mask design, the bottom contact is a disk with four rectangular arms that extend to allow actuation. The $\mathrm{ZnO}$ layer is sputtered through a circular mask, while the top contact structure consists of equal sized dumbbell shaped contacts. Profiler measurements indicated that the $\mathrm{ZnO}$ layers had a total thickness of $5 \mu \mathrm{m}$. 


\subsubsection{Electrical}

While the mean electrical resistance measured between the top and bottom contacts was around $105 \mathrm{k} \Omega$, the minimum resistance was measured at $95 \Omega$. It is noteworthy to mention that excluding the contacts situated near the substrate edges, a resistance of at least $4 \mathrm{~K} \Omega$ was measured across the $\mathrm{ZnO}$ film thickness. This suggests that the lower measured resistance values at each substrate's edges were simply outliers, probably due to localized structural imperfections.

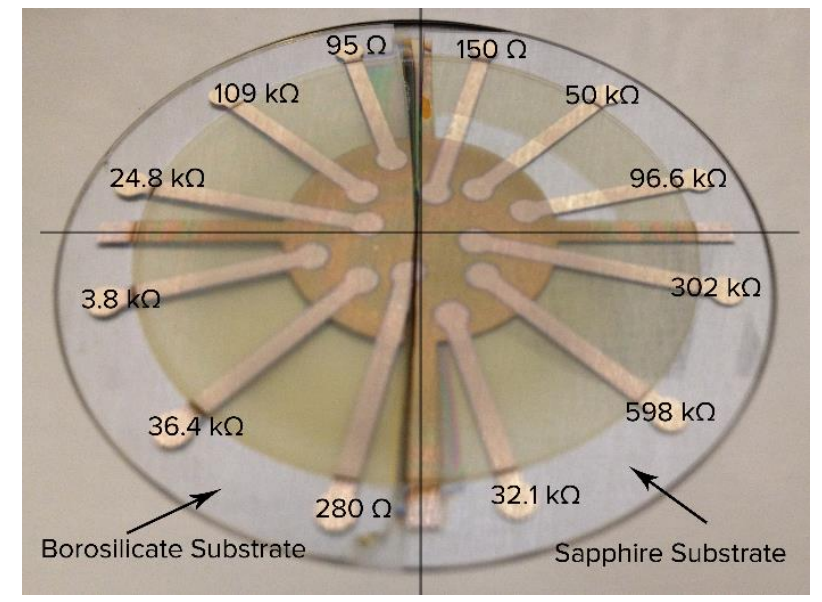

Figure 9.2: Resistance values across the $\mathrm{ZnO}$ film at each contact

\subsubsection{XRD}

X-ray diffraction analysis of both $5 \mu \mathrm{m}$ thick $\mathrm{ZnO}$ films indicated that growth on top of sapphire gave better results in terms of lower residual stress levels, both on the bottom contact layer and directly on the substrate, as seen in figure 9.3. A promising observation was the lack of dependence of the estimated residual stress levels on the $\mathrm{ZnO}$ layer thickness. For both substrate materials, the stress was significantly lower when the films were grown on top of the bottom contact. Hence, this recipe produced $5 \mu \mathrm{m}$ thick $\mathrm{ZnO}$ films with comparable 
residual stress levels to the submicron films presented in the studies of chapters 5 and 6.

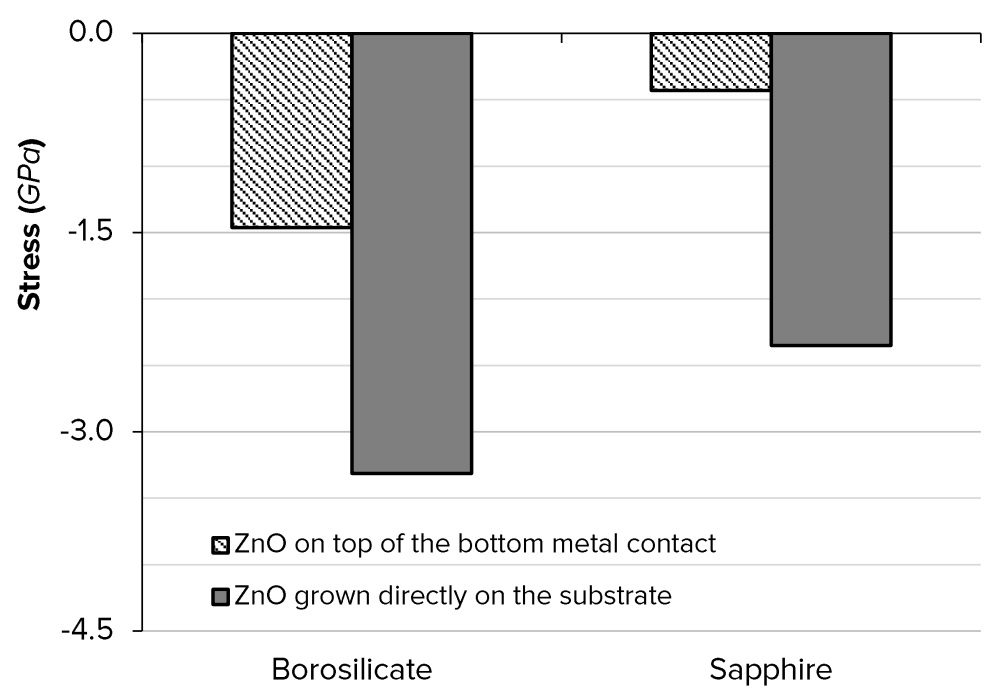

Figure 9.3: The estimated residual stress levels of the $5 \mu \mathrm{m} \mathrm{ZnO}$ layer deposited on both borosilicate and sapphire substrates.

As seen in figure 9.4, the estimated crystallite domain sizes from the $\mathrm{ZnO}(002)$ peaks were similar for films grown on both substrates, with no significant difference between sputtering on top of the bottom contact or directly onto the substrates.

Finally, the $\mathrm{ZnO}$ (002) selectivity was evaluated with respect to the highest measured undesirable orientation in each sample; i.e., the $\mathrm{ZnO}$ (100) orientation. This selectivity was found to be problematically low, particularly when the $\mathrm{ZnO}$ film was deposited on top of the bottom contact. In this latter case, the films were rather (100) oriented; thereby, unfit for our transducer application. Comparing both substrate materials, selectivity was slightly better on top of sapphire, as seen in figure 9.5. 


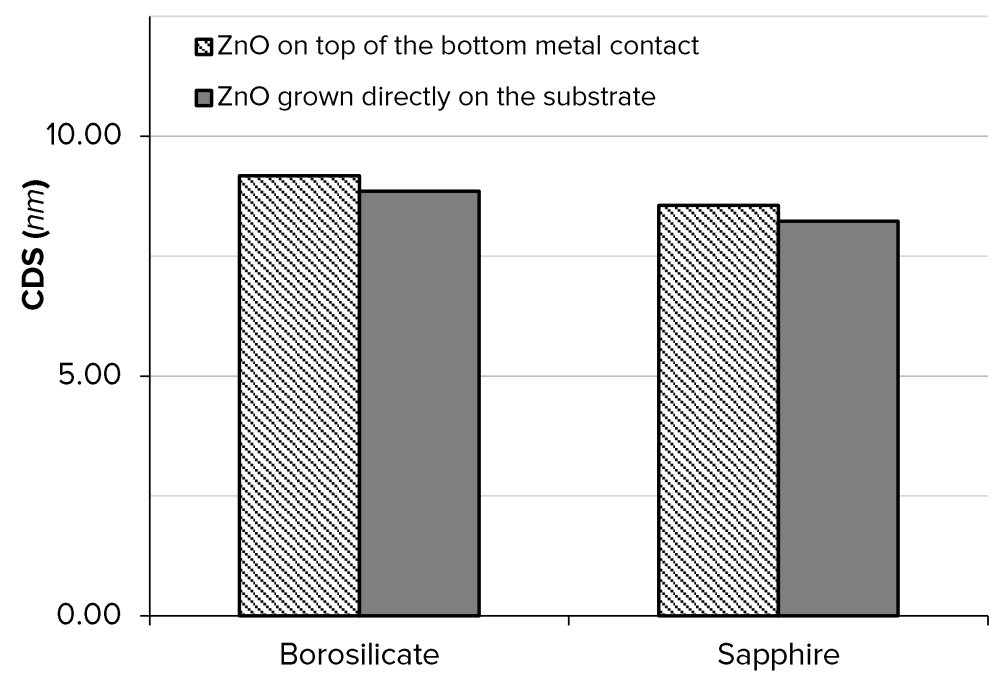

Figure 9.4: The estimated CDS values from the $\mathrm{ZnO}$ (002) peaks for films deposited on both substrates.

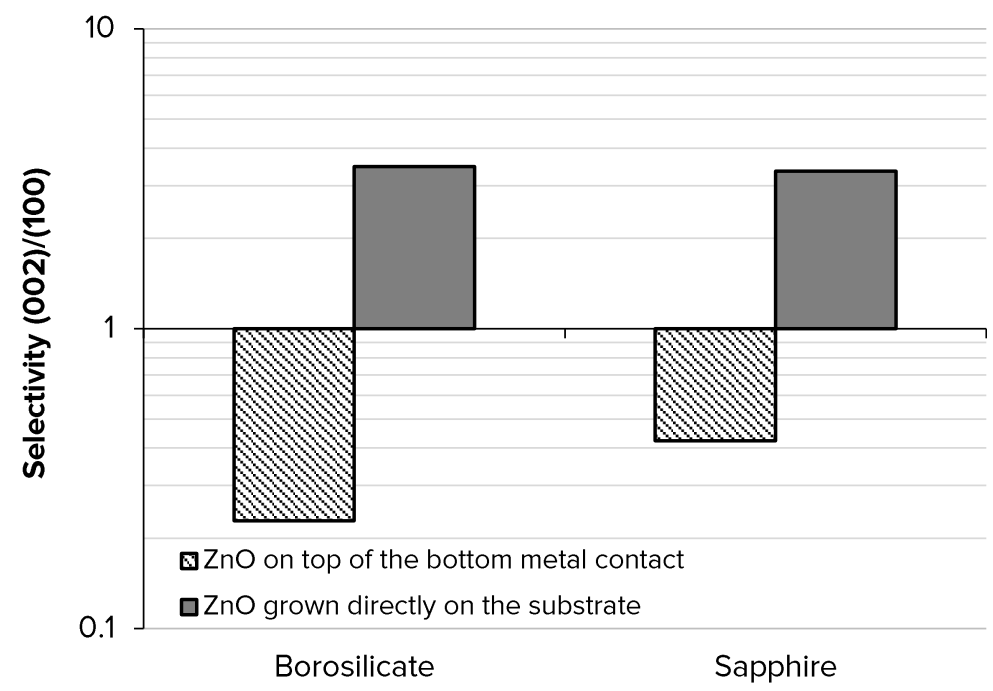

Figure 9.5: The (002)/(100) selectivity for the $5 \mu \mathrm{m} \mathrm{ZnO} \mathrm{films}$ grown on both borosilicate and sapphire substrates.

Therefore, despite our success in depositing structurally stable $\mathrm{ZnO}$ thick films with a thickness of at least $5 \mu \mathrm{m}$ and with acceptable stress levels and crystallite domain sizes, these films were not grown along the c-axis. This problem needed 
to be tackled before the fabrication process is considered suitable for creating the required c-axis oriented device-thick films.

\subsection{Second Structure Attempt}

Following the gold contact dewetting and the transparent conductive oxide studies, we attempted to create a fully transparent transducer structure using aluminium doped contacts. Thus, a two-inch c-axis sapphire wafer was used as a substrate for deposition. The bottom contact layer was sputtered at 200W using the $2 \%$ aluminium doped $\mathrm{ZnO}$ target, in an argon background with a 9sccm flowrate and a pressure of $3 \times 10^{-3}$ mbar. The substrate temperature was maintained at $275^{\circ} \mathrm{C}$, while the sample-target distance was $92 \mathrm{~mm}$. The substrate was placed 1 inch away from the target centre, to minimize the damage associated with energetic oxygen ions.

Upon reaching the required thickness, the chamber was left to cool down, to prepare for the undoped $\mathrm{ZnO}$ layer deposition. This layer was sputtered with the same settings used for depositing the bottom contact, except for having an ambient substrate temperature of $\approx 40^{\circ} \mathrm{C}$, and an RF power of 240W. This deposition lasted for 6 hours, to yield the required film thickness. In this process, the reasoning behind skipping the $\mathrm{ZnO}$ seed layer deposition and the in situ annealing steps was the assumption that the bottom contact layer would be $\mathrm{ZnO}$ (002) oriented, as supported by the results presented in section 8.3.1. Therefore, this bottom contact layer would act as a dense seed layer for $\mathrm{ZnO}$ crystal growth along the c-axis. Finally, the top contact layer was deposited using settings identical to the bottom contact sputtering process. The resulting sample came out visibly transparent and uniform, with no signs of flaking or peeling, as seen in figure 9.6. 


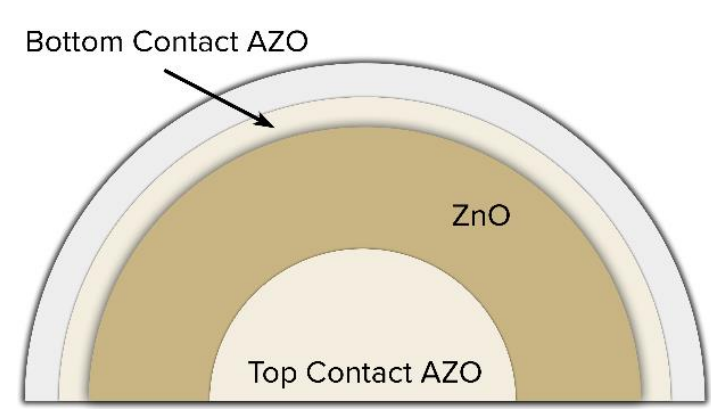

Wafer

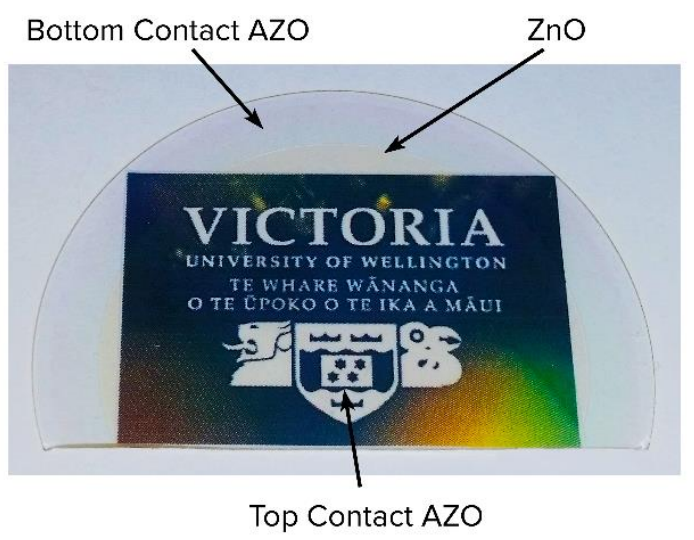

Top Contact AZO

Figure 9.6: (left) a sketch illustrating the top view of the device structure; (right) an image showing the resulting fully transparent device structure. A VUW logo has been placed under the sample to highlight its transparency.

Profiler measurements confirmed that this structure had a $\mathrm{ZnO}$ film thickness of $5 \mu \mathrm{m}$, while the aluminium doped $\mathrm{ZnO}$ bottom and top contacts had a thickness of 300nm each.

\subsubsection{UV/VIS Spectroscopy}

UV/VIS spectroscopy measurements confirmed the highly transparent nature of our deposited film structure. Several positions within our sample were inspected using the spectrometer, and similar optical transmission results were obtained. The recorded optical transmission measurement at the centre of the sample is shown in figure 9.7. 

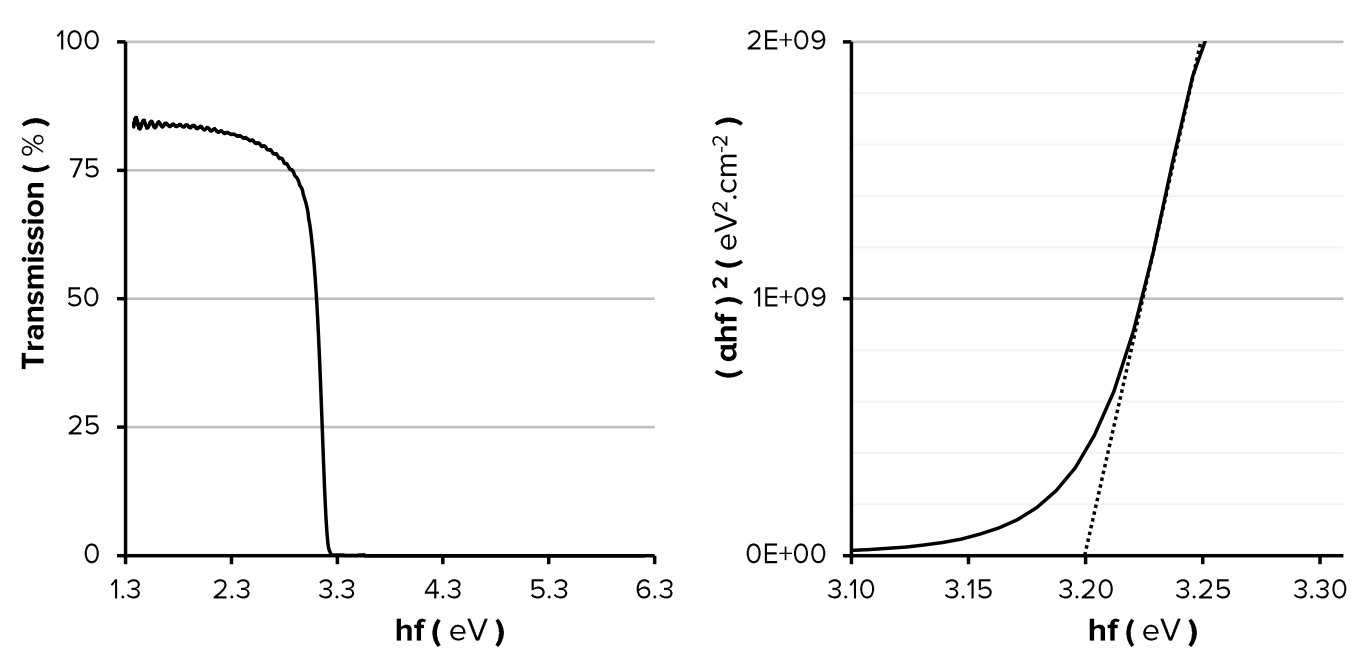

Figure 9.7: (left) Optical transmission through the $5 \mu \mathrm{m}$ thick $\mathrm{ZnO}$ film; (right) the corresponding Tauc plot showing a bandgap of $3.2 \mathrm{eV}$.

As seen in this figure, the absorbance edge through the whole device structure was estimated at $3.2 \mathrm{eV}$, while optical transmission was consistently larger than $80 \%$ for photons having a lower energy. The sample was completely opaque for shorter light wavelengths (higher energies).

\subsubsection{XRD}

As the whole device structure was zinc oxide based, it was important to reduce the $x$-ray spot size to accurately characterize the properties of each layer separately. At $40 \mathrm{kV}$, the $\mathrm{x}$-ray beam is expected to penetrate the whole sample, which makes the characterization of the top contact layer imprecise, as it is relatively thin and entirely located on top of the other two layers.

XRD measurements revealed a large disparity between the properties of the bottom contact layer, and the thick $\mathrm{ZnO}$ and the top contact layers. For instance, the estimated (002) crystallite domain size was around $23 \mathrm{~nm}$ for the 
bottom contact layer, while it was less than $5 \mathrm{~nm}$ for both undoped $\mathrm{ZnO}$ layer and the top contact layer, as seen in figure 9.8.

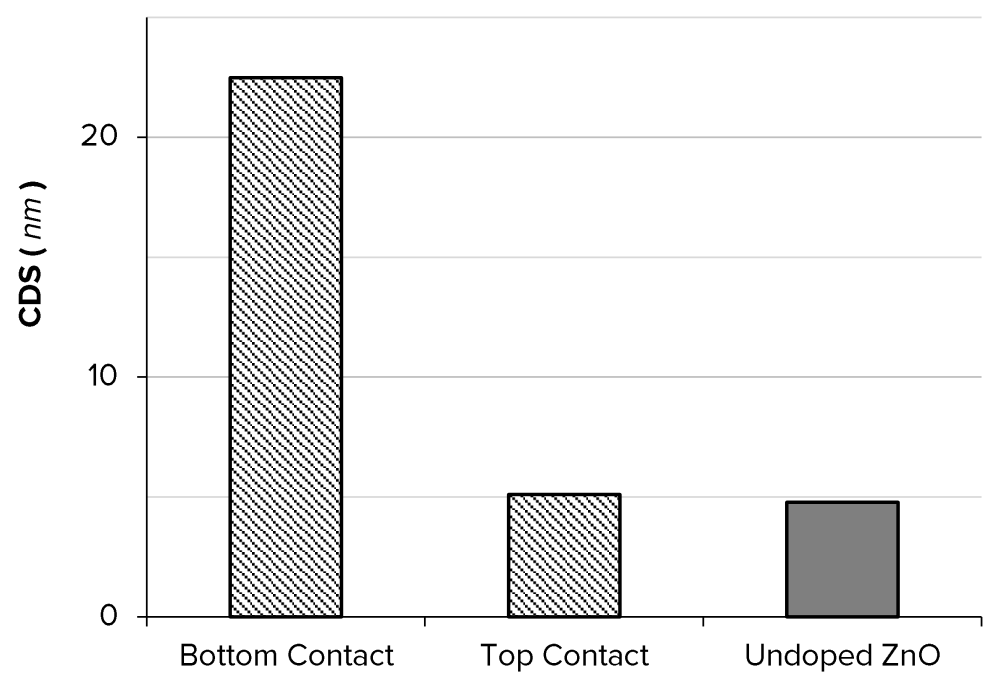

Figure 9.8: The CDS values estimated from the $\mathrm{ZnO}$ (002) peaks for each layer of the device structure.

The more critical difference concerned the crystal orientation of the different layers of the structure, as shown in figure 9.9. Thus, despite having a highly $\mathrm{ZnO}(002)$ oriented bottom contact layer, the $5 \mu \mathrm{m}$ undoped layer was found to be rather (100) and (101) oriented. This selectivity problem was resonant with the previously achieved result in section 9.2.2. Finally, the top contact layer was also measured to be (100) and (101) oriented. 


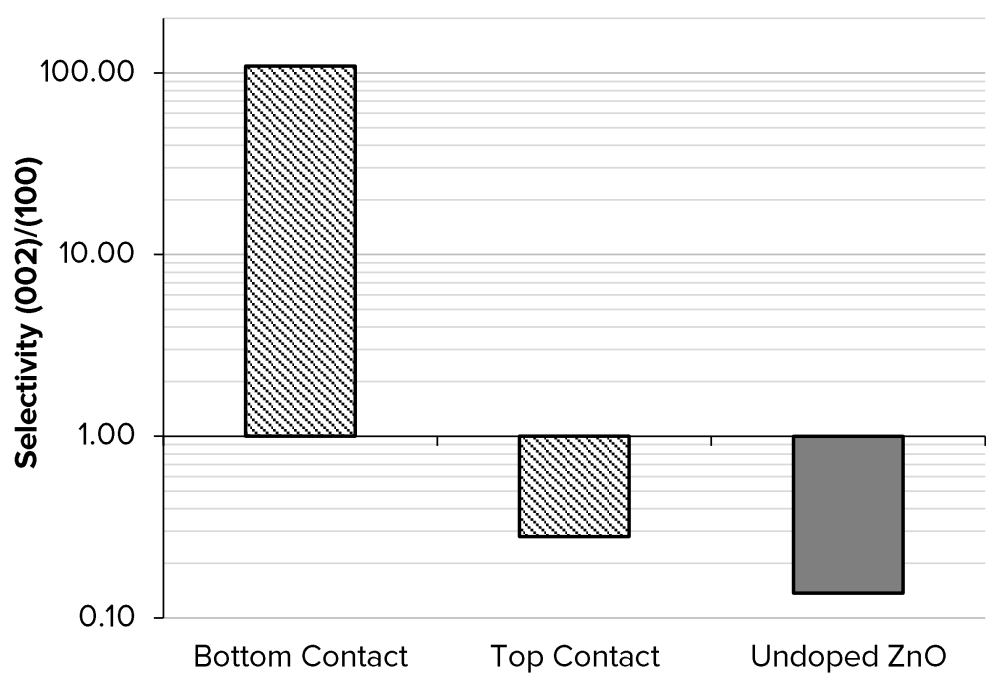

Figure 9.9: The $(002) /(100)$ selectivity for the different layers of the $\mathrm{ZnO}$ based device structure.

Accordingly, the zinc oxide's c-axis was perpendicular to the substrate in the initial growth stage, driven by surface energy minimization due to the large surface to volume ratio. However, as the film thickness increased to several microns, the (100) and the (101) orientations became dominant, which indicates that other factors start to dictate the growth dynamics. Lee et al. [205] argues that the energetic oxygen ion bombardment during $\mathrm{ZnO}$ growth chiefly affects the most packed orientation; i.e., the (002) texture. Thus, the loosely packed crystallites, such as the (101) and (110) grow less disturbed and act as seeds for further growth. In addition, they report that their (002) films changed to a mix of (002) and (101) when the thickness was between 3-6 $4 \mathrm{~m}$. Finally, the (110) diffraction peak became predominant at $7 \mu \mathrm{m}$. Thus, $\mathrm{ZnO}$ growth during sputtering changed from columnar below $1 \mu \mathrm{m}$, to elongated at $3 \mathrm{um}$, and finally faceted at $7 \mathrm{um}$.

As our proposed device structure relies on having a c-axis oriented zinc oxide layer for operation, and since our achieved results were in agreement 
with the aforementioned arguments regarding the dependence of the (002) texture orientation on the film thickness, the next evident goal was to produce a $\mathrm{ZnO}$ growth recipe that counters the unwanted film growth factors, and yields the required thick c-axis oriented columnar films.

\subsection{Cyclic Growth Study}

Our sputtering process conditions successfully produced one micron thick caxis oriented zinc oxide films on top of dense wafers. This is attributed to the high surface area to volume ratio within this thickness regime, which makes the drive for surface energy minimization to be the dominant growth factor. Accordingly, the most packed wurtzite arrangement; i.e., the (002) orientation is expected to dominate the growth texture. However, as the film grows thicker, surface energy minimization becomes less significant, and other variables such as oxygen ion induced damage and surface defects gradually become more important. This priority change was identified as the main culprit to our crystal orientation problem; thereby, the following study was initiated.

Here, we aimed to evaluate the effects of thermal annealing on the $\mathrm{ZnO}$ film's densification through the reduction of its surface and strain energy [213], which can occur within the solid-state at temperatures well below the material's melting point [214]. By having a denser film structure through thermal annealing, we hope to reinstate the initial growth factors' priorities, whereby the annealed $\mathrm{ZnO}$ film would act as a dense substrate to the next deposited $\mathrm{ZnO}$ layer. In other words, we wanted to deposit a $1 \mu \mathrm{m}$ thick $\mathrm{ZnO}$ film, which we know should be c-axis oriented. Then, sputtering is paused to allow film densification by thermal annealing. Next, the substrate is allowed to cool down to the initial set point, before the process is started all over again. Therefore, the 
required $\mathrm{ZnO}$ film thickness is grown in $1 \mu \mathrm{m}$ cycles, where each cycle consists of a sputtering step and a thermal annealing step.

As this study aimed to compare the conventional undoped zinc oxide sputtering process used in section 9.3 to the aforementioned cyclic deposition technique, two identical c-axis sapphire wafers were used as substrates for deposition.

On both wafers, the bottom contact layer was sputtered at 200W using the $2 \%$ aluminium doped $\mathrm{ZnO}$ target, in an argon background with a $9 \mathrm{sccm}$ flowrate and a pressure of $3 \times 10^{-3}$ mbar. The substrate temperature was maintained at $275^{\circ} \mathrm{C}$, while the sample-target distance was set at $92 \mathrm{~mm}$. The substrate was placed 1 inch away from the target centre, to minimize the damage associated with energetic oxygen ions. Upon reaching the required thickness, the chamber was left to cool down, to prepare for the undoped $\mathrm{ZnO}$ deposition for each sample.

The first sample was sputtered using the conventional single-step deposition. Sputtering was carried out with the same settings used for depositing the bottom contact, except for having an ambient substrate temperature of $\approx 40^{\circ} \mathrm{C}$. This deposition lasted for 5 hours, to yield the required film thickness of $4 \mu \mathrm{m}$. Again, the bottom contact layer is assumed to be a good seed layer for $\mathrm{ZnO}$ crystal growth along the c-axis.

The second sample was sputtered using the proposed cyclic deposition method. Therefore, the process was initiated by sputtering a $1 \mu \mathrm{m}$ thick $\mathrm{ZnO}$ film using identical settings to the first sample. This was followed by a one hour annealing step at $325^{\circ} \mathrm{C}$, in an oxygen background with a $2 \mathrm{sccm}$ flow rate. Then, the chamber was allowed to cool back to room temperature, to prepare for the next cycle. This deposition cycle was repeated three times, yielding a $3 \mu \mathrm{m}$ thick 
$\mathrm{ZnO}$ film. It should be noted that a slightly smaller mask was applied for each cycle, to ensure having an exposed inspection area, as seen in figure 9.10.
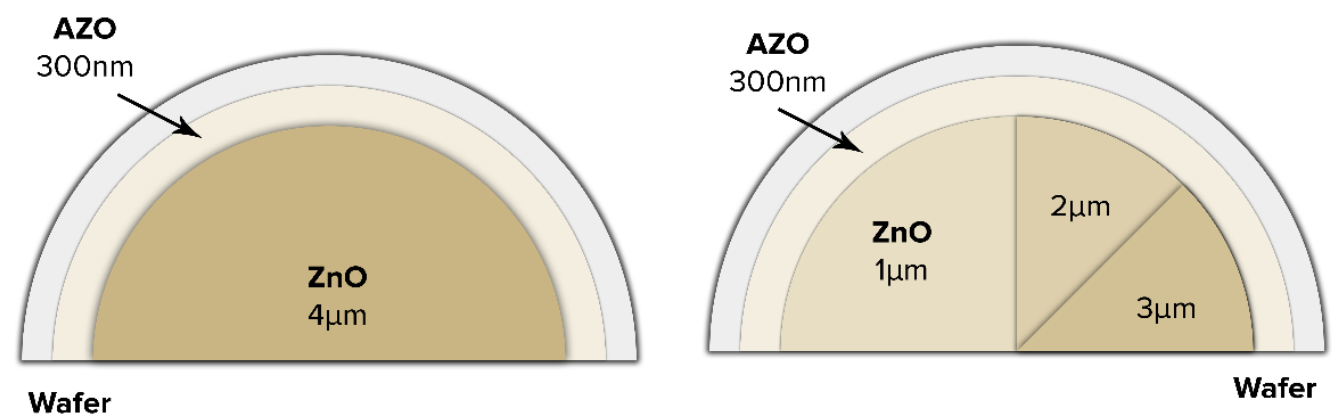

Figure 9.10: A sketch illustrating the mask design for the single deposition sample and the cyclic deposition sample included in this study.

Profiler measurements confirmed that the single deposition sample had a thickness of $4 \mu \mathrm{m}$, while the cyclic deposition sample had three regions, with a thickness of 1,2, and $3 \mu \mathrm{m}$, respectively.

\subsubsection{XRD}

The estimated crystallite domain sizes from the $\mathrm{ZnO}(002)$ peaks indicated that cyclic deposition consistently resulted in larger domain sizes, in the range of $10 \mathrm{~nm}$, for each of the 1,2 , and $3 \mu \mathrm{m}$ thick regions of the film. In comparison, the first sample that was deposited in a single step had a $\mathrm{ZnO}$ (002) crystallite domain size of $5 \mathrm{~nm}$, as seen in figure 9.11. 


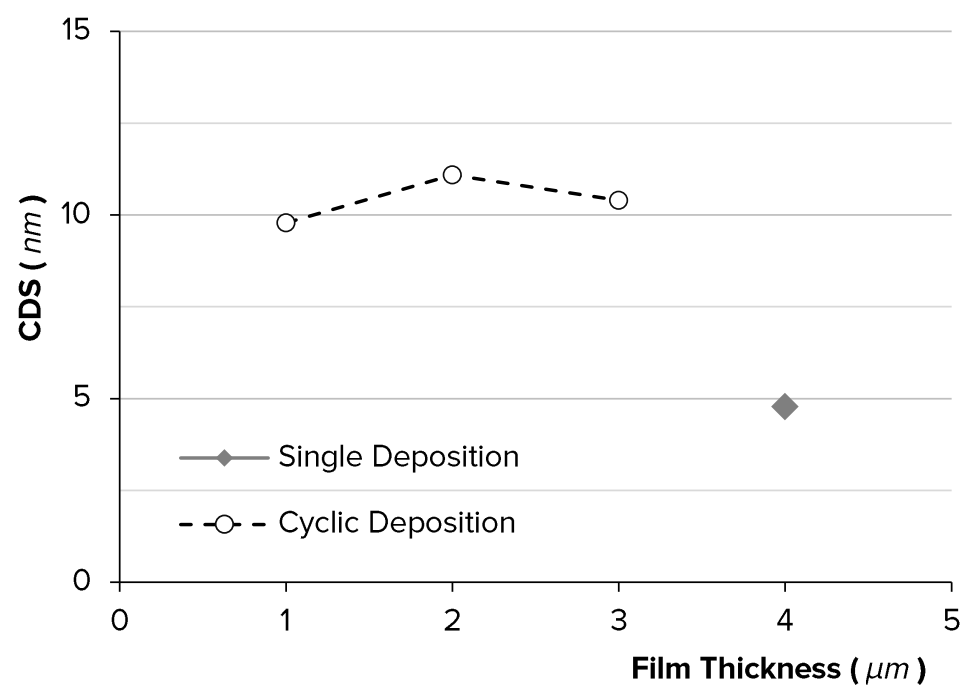

Figure 9.11: The average $\mathrm{ZnO}(002)$ crystallite domain size in each region of the cyclic deposited film, and the single step deposited film.

As the sputtering conditions were kept identical during both processes, the measured domain size disparity is attributed to the one hour annealing step at $325^{\circ} \mathrm{C}$ within each growth cycle.

The most significant result of this study was the successful deposition of a $3 \mu \mathrm{m}$ thick c-axis oriented $\mathrm{ZnO}$ film using the cyclic deposition approach, as shown in figure 9.12. Thus, while the conventional single deposition approach resulted in a (100) and (101) oriented $4 \mu \mathrm{m} \mathrm{ZnO}$ film, the cyclic deposition approach resulted in a $3 \mu \mathrm{m}$ thick (002) textured film. The measured $(002) /(100)$ selectivity for the cycle deposited film at the 1,2 , and $3 \mu \mathrm{m}$ layer surfaces was 5 , 14 , and 11 , respectively. 

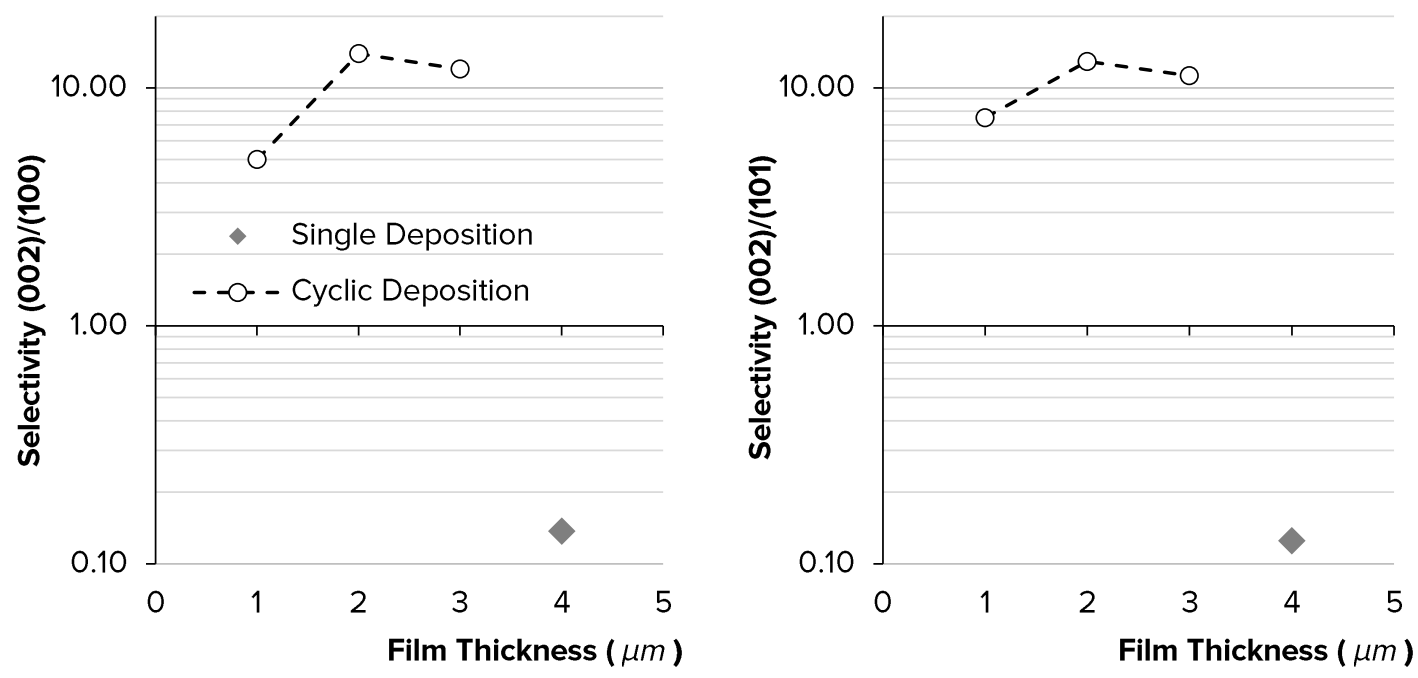

Figure 9.12: (left) the (002)/(100) selectivity and (right) the $(002) /(101)$ selectivity for the single deposited $4 \mu \mathrm{m} \mathrm{ZnO}$ film, and the $3 \mu \mathrm{m}$ cycle deposited $\mathrm{ZnO}$ film.

This favourable selectivity is almost two orders of magnitude higher than what is possible using the single deposition step. Consequently, cyclic deposition presents a realistic RF magnetron sputtering technique to fabricate $\mathrm{ZnO}$ films that satisfy all the structural requirements to develop our desired device structure.

\subsubsection{SEM}

SEM examination revealed stark morphological differences between the zinc oxide films of each sample, as seen in figure 9.13. The first $\mathrm{ZnO}$ film deposited in a single step was found to have a random cotton like morphology. Nonetheless, grain boundaries within the first $\sim 1.5 \mu \mathrm{m}$ were generally more uniform and aligned in a direction perpendicular to the substrate, indicating caxis oriented growth. As the film became thicker, the grains seemed to have merged and grew in all directions, indicating a Volmer-Weber like growth 
mode [24] (section 3.2.1.1). This thickness dependent morphological change is confirmed by other sources [205, 234, 235], and is attributed to energy loss minimization per unit area, in a response to the negative ion bombardment and the decreasing surface to volume ratio.

In contrast, SEM images showed a highly uniform and columnar nature of the $\mathrm{ZnO}$ film that was prepared using the cyclic deposition process. Such a columnar microstructure is associated with growth along the c-axis direction, which was confirmed in the XRD measurements shown in figure 9.12.
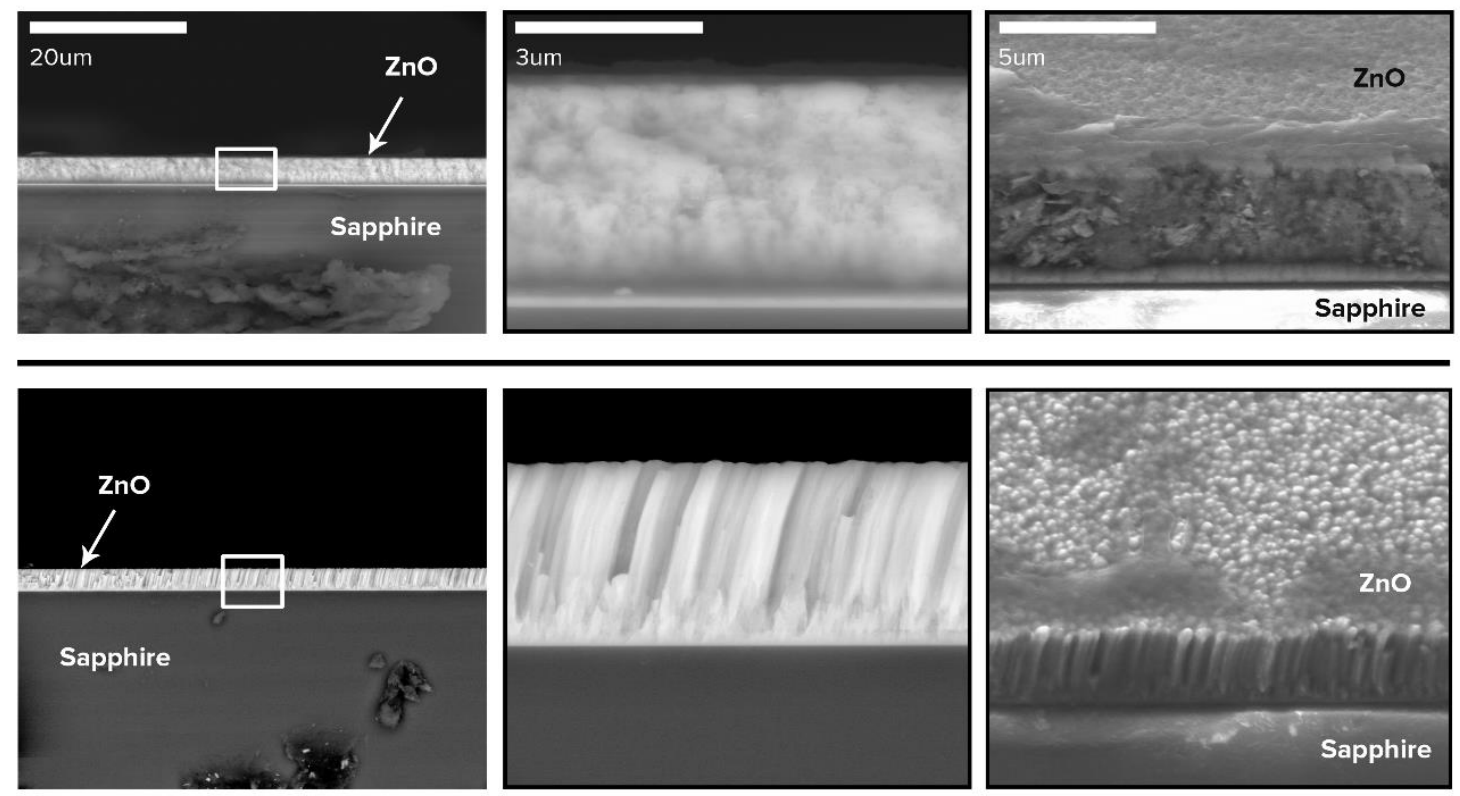

Figure 9.13: Cross-sectional and $20^{\circ}$ tilted secondary electrons SEM micrographs of both $\mathrm{ZnO}$ samples, highlighting the morphological differences between the single step deposited film (top), and the cycle deposited film (bottom).

Such film qualities are highly desirable for our transducer application, as uniformity and correct orientation are expected to maximize the piezoelectric response along the thickness of the transducer. 
Further interesting SEM images were taken off the sample's edge, where the $\mathrm{ZnO}$ film was occasionally broken in a way that uncovered the $1 \mu \mathrm{m}$ layers associated with each cycle, as seen in figure 9.14.
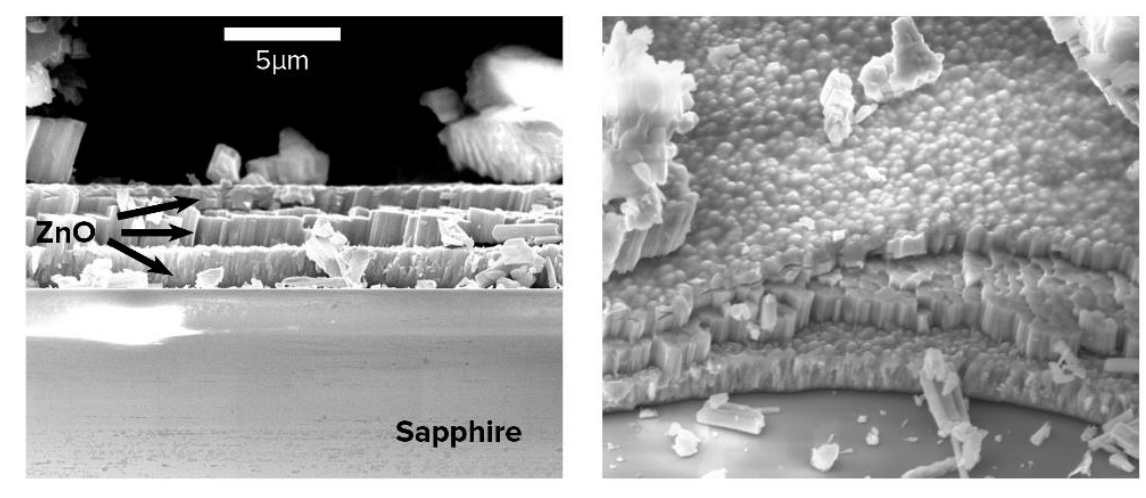

Tilt

$45^{\circ}$

Figure 9.14: Cross-sectional and $45^{\circ}$ tilted secondary electrons SEM micrographs of the cycle deposited sample. The different $1 \mu \mathrm{m} \mathrm{ZnO}$ layers are clearly distinguishable.

Bright lines exist between the layers, suggesting a relatively large interface area. In addition, it is revealed through the visible layers' lamination that the binding force between the grains of each layer is stronger than the binding force between the adjacent layers. Both observations point to a successful densification of each $\mathrm{ZnO}$ layer.

\subsection{Third Structure Attempt}

After the doped and undoped $\mathrm{ZnO}$ sputtering processes were optimized to allow the creation of films having the required structural, optical, and electrical properties for the different layers of the proposed device structure (chapter 5), 
the next step was to attempt the creation of a single element transducer structure. The top and bottom contact masks were altered to allow probing using ground-signal-ground probes. These probes are required for the accurate electrical characterization at such a frequency range $(300 \mathrm{MHz}$ to $1 \mathrm{GHz})$, since the wavelength is typically shorter than the circuit's dimensions.

A two-inch c-axis sapphire wafer was used as a substrate for deposition. The bottom contact layer was sputtered at 200W using the $2 \%$ aluminium doped $\mathrm{ZnO}$ target, in an argon background with a 9sccm flowrate and a pressure of $3 \times 10^{-3}$ mbar. The substrate temperature was maintained at $275^{\circ} \mathrm{C}$, while the sample-target distance was $92 \mathrm{~mm}$. The substrate was placed 1 inch away from the target centre, to minimize the damage associated with energetic oxygen ions. Upon reaching the required thickness, the chamber was left to cool down, to prepare for the undoped $\mathrm{ZnO}$ layer deposition.

The undoped $\mathrm{ZnO}$ film was sputtered using the aforementioned cyclic deposition method, where the sputtering parameters were identical to that of the second sample in the previous study, presented in section 9.4. Again, the deposition cycle was repeated three times, yielding a $3 \mu \mathrm{m}$ thick $\mathrm{ZnO}$ film.

Finally, the top contact layer was deposited using settings identical to the bottom contact sputtering process. The resulting sample came out visibly transparent and uniform, with no signs of flaking or peeling, as seen in figure 9.15 .

It should be noted that photolithography was used to pattern the different layers of this device structure, and a photoresist spacer layer was embedded in the device structure to allow future electrical probing.

Profiler measurements confirmed that the contacts had a thickness of $300 \mathrm{~nm}$ while the undoped $\mathrm{ZnO}$ film had a total thickness of $3 \mu \mathrm{m}$. 

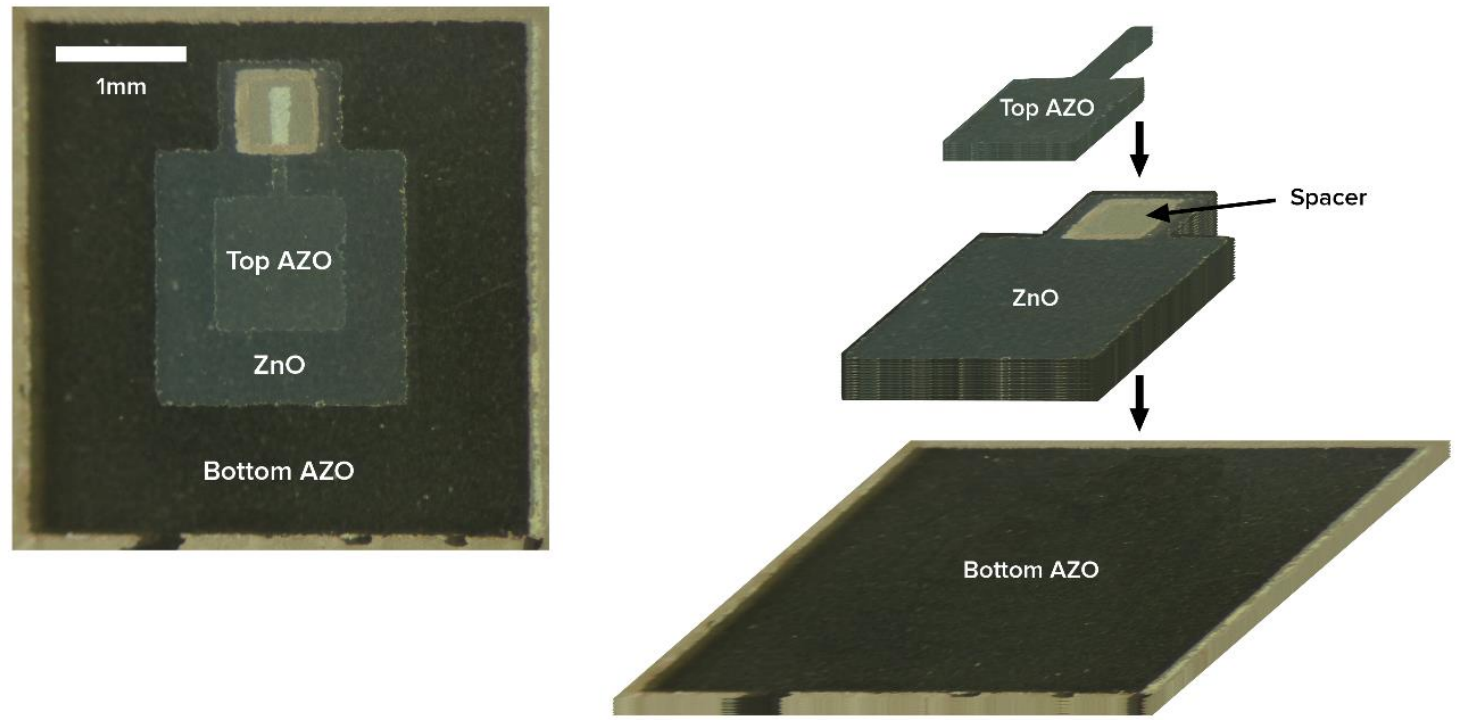

Figure 9.15: An image showing our transparent single element structure, photographed on top of a black paper. For further clarification, a 3D digital reconstruction is shown to the right.

\subsubsection{UV/VIS Spectroscopy}

UV/VIS spectroscopy was used to evaluate the optical transparency of our single element structure. The transmission and absorption plots are presented in figure 9.16.

It can be seen that the structure is transparent, with a transparency around $40 \%$ until the absorbing edge. The estimated absorption edge for photons passing through the whole element structure is at $3.2 \mathrm{eV}$. 

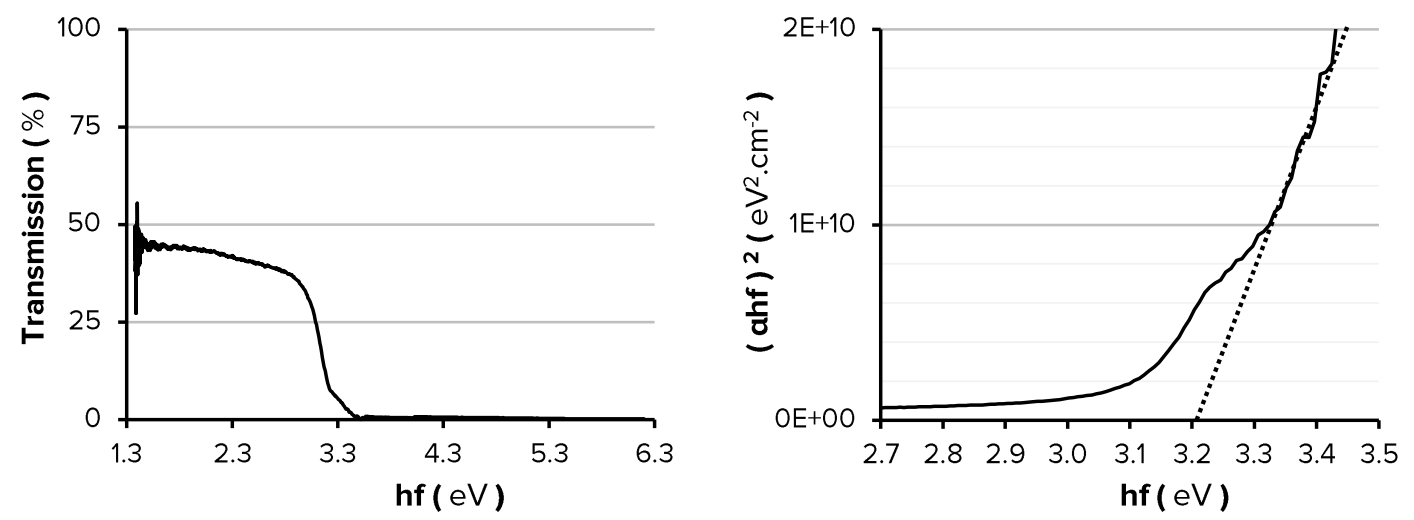

Figure 9.16: (left) Optical transmission through the single element structure; (right) Tauc plot showing a bandgap of 3.2eV

Note that transmission through this sample was almost half of that of the conventionally deposited structure described in section 9.3. This decrease in transmission could be attributed to reflection and scattering effects at the different interfaces of the structure, particularly between the multiple $1 \mu \mathrm{m}$ layers. In addition, a non-transparent photoresist spacer layer was included in the structure as seen in figure 9.15, which can contribute to the decrease in transmission, depending on the spectrometer's spot-size.

\subsubsection{XRD}

XRD measurements revealed results similar to the previous section (section 9.4.1), with an estimated average CDS of $9.5 \mathrm{~nm}$. The most significant result was the confirmed (002) selectivity, where the (002):(101) selectivity was 7.3, while the (002):(100) selectivity was 8.2. These results indicate that our $3 \mu \mathrm{m}$ thick device structure has suitable structural and electrical properties, which satisfy the theoretical conditions for developing a piezoelectric $\mathrm{ZnO}$ film. 


\subsection{Chapter Summary}

- The metal contacts were not suitable for developing the required transducer structure, as poor (002) selectivity resulted, and they were found unsuitable for the critical post-growth anneal process.

- The cyclic growth study was successful in creating thick and (002) oriented $\mathrm{ZnO}$ films.

- An all-ZnO transparent single element transducer structure was developed. 


\section{CHAPTER 10}

\section{Discussions and Conclusions}

The fabrication of thin piezoelectric films that can operate in the thickness mode at frequencies higher than $300 \mathrm{MHz}$ presents several challenges, as the films must maintain their transducing properties at small and reproducible geometric dimensions, possess enough structural integrity and a high degree of uniformity to allow reliable operation, and permit matched acoustic and electrical coupling to their driving circuits through conductive contacts. Our targeted transducer structure that aims for the $300 \mathrm{MHz}$ to $1 \mathrm{GHz}$ frequency range requires process optimization to push the limits of technology, which presents a complex task that is met by many challenges, and is highlighted through the lack of commercial transducers operating within this frequency range.

Over the course of this work, we have optimized the microfabrication process to pave the way for creating high-frequency ultrasound transducers, with operating frequencies exceeding $300 \mathrm{MHz}$, and up to $1 \mathrm{GHz}$. Given its 
versatile and suitable properties, zinc oxide was selected to make our active layer, while several materials were investigated to construct the relevant ohmic contacts. The processes combine radio frequency magnetron sputtering and thermal annealing, and make it possible to reproducibly create reliable, relatively cheap and mechanically stable thin-films, with final thicknesses exceeding $5 \mu \mathrm{m}$, and having preferential crystal structure and orientation, which are essential for a practical piezoelectric response.

The conclusions and recommendations achieved throughout this study are summarized in the following sections.

\subsection{Sputtering Optimization}

The general fabrication process was described, and the relevant parameters were identified. Previous VUW results were summarized, where it was found that c-axis oriented films were achieved at a relatively high deposition rate of $15 \mathrm{~nm} / \mathrm{min}$. RF power exceeding $240 \mathrm{~W}$ was found inappropriate for producing (002) oriented films, substrate heating contributed to having unwanted orientations, and setting the pressure lower than 1.1E-2 mbar resulted in highly stressed and peeling films. Introducing oxygen to the sputtering gas mixture gave rise to unwanted orientations. Accordingly, a four-step deposition process was recommended, to enhance the films crystal properties while maintaining a moderate deposition rate. Thereafter, the sputtering related studies carried out throughout this work were presented. It was found that chromium formed an essential interface layer between the gold contacts and the $\mathrm{ZnO}$ film. Having thinner gold bottom contacts of $20 \mathrm{~nm}$ allowed $\mathrm{ZnO}$ films on top to grow with a better (002) selectivity. The thickness uniformity of the sputtered $\mathrm{ZnO}$ as a function of the substrate to target distance and placement was found acceptable 
given the selected distances and the stationary sample holder, where thickness variation within a $1 \mathrm{~cm}$ device was expected to be of the order of $2 \%$. Substrate heating and oxygen-containing sputtering gas mixture were confirmed to be precursors of unwanted orientations on top of borosilicate and sapphire wafers.

\subsection{Thermal Annealing Effects on the ZnO Layer}

It was indicated that both in situ and post-growth external annealing steps were necessary to improve the films' crystal properties.

The results of this chapter highlighted the importance of having an oxygen-rich environment during the in-situ anneal step of sputtered $\mathrm{ZnO}$ films. This simple factor helped us to create films having low stress, large crystallite domain sizes, (002) texture and high electrical resistance. A lowering of the relative oxygen concentration during this step caused the undesired crystal orientations to dominate, which is detrimental to our piezoelectric application. Compressive stress inherent in as-grown films was eliminated by annealing at a temperature of $400^{\circ} \mathrm{C}$; however, annealing at $800^{\circ} \mathrm{C}$ was needed to ensure having a dominant (002) textured film. It is suggested that the in-situ anneal in an oxygen atmosphere aids the elimination of oxygen vacancies which are formed during the sputtering process. The elimination of these vacancies was found essential to ensure favouring the desired (002) texture during recrystallization 


\subsection{Metal Contact Dewetting due to Annealing}

Our measurements confirmed the optimization of the $\mathrm{ZnO}$ films with annealing, where the average grain diameter increased from $\approx 50 \mathrm{~nm}$ for the assputtered films to $\approx 150 \mathrm{~nm}$ when annealed at $800^{\circ} \mathrm{C}$. The residual stress of the (002) textured $\mathrm{ZnO}$ films was eliminated by heating at temperatures $\geq 400^{\circ} \mathrm{C}$, and the $\mathrm{ZnO}(002)$ crystallite domain size increased from $8 \mathrm{~nm}$ as sputtered to $45 \mathrm{~nm}$ when heated at $800^{\circ} \mathrm{C}$.

Such active layer structural optimization however was accompanied by a gradual degradation of the $\mathrm{Cr} / \mathrm{Au} / \mathrm{Cr}$ contact structure underneath due to dewetting. AFM, SEM, and EDS imaging indicated void initiation at $400^{\circ} \mathrm{C}$, after which void growth proceeded, and coalescence was dominant at $800^{\circ} \mathrm{C}$, resulting in Au-Cr particles having an average diameter $\approx 1.5 \mu \mathrm{m}$. This agglomeration process was accelerated in areas where the $\mathrm{ZnO}$ overlayer was absent, leading to more dispersed and smaller particles with diameters of $\approx 500 \mathrm{~nm}$ at $800^{\circ} \mathrm{C}$. XRD measurements indicated an exponential increase in the $\mathrm{Au}$ (111) reflection intensity as the annealing temperature increased. The $\mathrm{Au}$ (111) crystallite domain size increased from 6nm -as deposited- to 31nm after annealing at $800^{\circ} \mathrm{C}$. Electrical measurements indicated that the resistance of the $\mathrm{Cr} / \mathrm{Au} / \mathrm{Cr}$ films increased from $\approx 100 \Omega$ for the as deposited films to $\approx 10 \mathrm{M} \Omega$ at $400-600^{\circ} \mathrm{C}$ for films capped with a $\mathrm{ZnO}$ layer, and up to an open circuit at $800^{\circ} \mathrm{C}$. As to the areas that had no $\mathrm{ZnO}$ on top, an open circuit was measured for temperatures $\geq 400^{\circ} \mathrm{C}$.

Thus, we report severe agglomeration and dewetting of thin gold films occurring at temperatures well below the melting point. This process was found to be slower yet similar when the metal films were encapsulated with a thicker 
zinc oxide layer. Based on these findings, the highest recommended annealing temperature before agglomeration of the metal contact layer was $400^{\circ} \mathrm{C}$.

\subsection{Transparent Conductive Oxide Contacts}

This chapter described the processing study that aimed to produce transparent and conductive electrical contacts for our transducer structure. Thus, gallium doped and aluminium doped zinc oxide films were deposited using RF magnetron sputtering. At the onset of this study, the films were neither conductive, $\mathrm{ZnO}$ (002) oriented, nor fully transparent. Therefore, the process was gradually adjusted to improve the quality of the resulting samples. A main problem throughout this study was the lack of uniformity across a film's surface, where a concentric damage pattern due to oxygen ion bombardment was visible. This damage was mostly located at the areas facing the plasma centre, as well as the eroded racetrack within the target, which meant that surface damage had an alternating profile, with the sample's surface divided into 4 distinguishable regions. The damaged regions 1 and 3 suffered from lower $\mathrm{ZnO}$ (002) selectivity and optical transparency, and higher electrical resistivity. On the other hand, the outermost region within each sample; i.e.,

region 4, had the best optical, electrical, and crystal properties. The size and severity of this damage pattern depended on several sputtering parameters, particularly the RF power, the sputtering pressure, substrate temperature, and sample-target distance.

Accordingly, surface damage was minimized in our sputtering system by limiting the RF power to $200 \mathrm{~W}$, and using the maximum possible substrate temperature of $325^{\circ} \mathrm{C}$, and the largest feasible sample-target distance of $92 \mathrm{~mm}$. 
In addition, off-axis sputtering was utilized to minimize the energetic oxygen ion bombardment; thereby, maximizing the area of region 4 .

These optimal conditions enabled us to consistently deposit conductive (resistivity $\approx 0.3 \Omega . \mathrm{cm})$ and transparent (75\% transparency) doped zinc oxide films, with a high $\mathrm{ZnO}(002)$ selectivity (>200) and unnoticeable surface damage.

\subsection{Device Thickness Films}

This chapter documented our attempts for creating device thickness film structures at the different project stages. These structures had a $\mathrm{ZnO}$ layer thickness exceeding $3 \mu \mathrm{m}$ and were mostly deposited on top of a bottom contact layer.

The first attempt was conducted following the studies reported in chapters 5 and 6 . Thus, a $5 \mu \mathrm{m}$ thick $\mathrm{ZnO}$ film was sputtered on top of a $\mathrm{Cr} / \mathrm{Au} / \mathrm{Cr}$ bottom contact, using both sapphire and borosilicate wafers. The resulting films were uniform, with acceptable stress levels. However, the film suffered from a very poor $\mathrm{ZnO}(002)$ selectivity.

The second attempt was almost identical to the first attempt, except for the bottom contact layer, which was deposited from an aluminium doped zinc oxide target. The resulting sample was highly transparent and uniform. However, despite having a highly c-axis oriented bottom contact layer, the undoped $\mathrm{ZnO}$ layer was (100) and (101) oriented.

Consequently, a sputtering study was carried out, to isolate the mechanisms that dictate the favourable orientations during $\mathrm{ZnO}$ crystal growth. This study was largely successful, and identified alternate sputtering and annealing processes to remedy the (002) selectivity problem. Accordingly, a 
$3 \mu \mathrm{m}$ thick c-axis oriented $\mathrm{ZnO}$ film was grown on top of an $\mathrm{AZO}$ bottom contact.

Finally, an attempt to fabricate a fully transparent single element transducer using the developed processing recipe was made. The attempt was successful, and relied on photolithography to pattern the developed structure. As the all-ZnO structure was transparent, and c-axis oriented, there was an urgent interest for proposing an integrated optical-acoustic imaging system that would open the window for creating a novel dual microscope that uses both ultrasound and optical imaging to inspect samples.

\subsection{Integrated Optical-Acoustic Imaging}

The use of transparent conductive $\mathrm{ZnO}$ layers to replace the traditional metal contacts has been shown to enable the use of relatively high post-growth anneal temperatures and thus improve the quality of the active $\mathrm{ZnO}$ films. In addition, it also serves as a lattice-matched buffer layer, thus facilitating the deposition of (002) oriented films.

In addition, an even more important advantage that can be gained from the use of these contact layers may be in the ability to build a transducer structure with both optical and acoustical imaging ability. The inability of conventional acoustical microscopes to simultaneous form an optical image makes for a slow process in alignment and scanning of the acoustic beam on the desired sample area. The ability to simultaneously obtain both images may then facilitate the use of acoustic microscopy.

A first design of such an integrated optical acoustical microscope lens based on a $\mathrm{AZO} / \mathrm{ZnO} / \mathrm{AZO}$ device structure is shown in figure 10.1. 


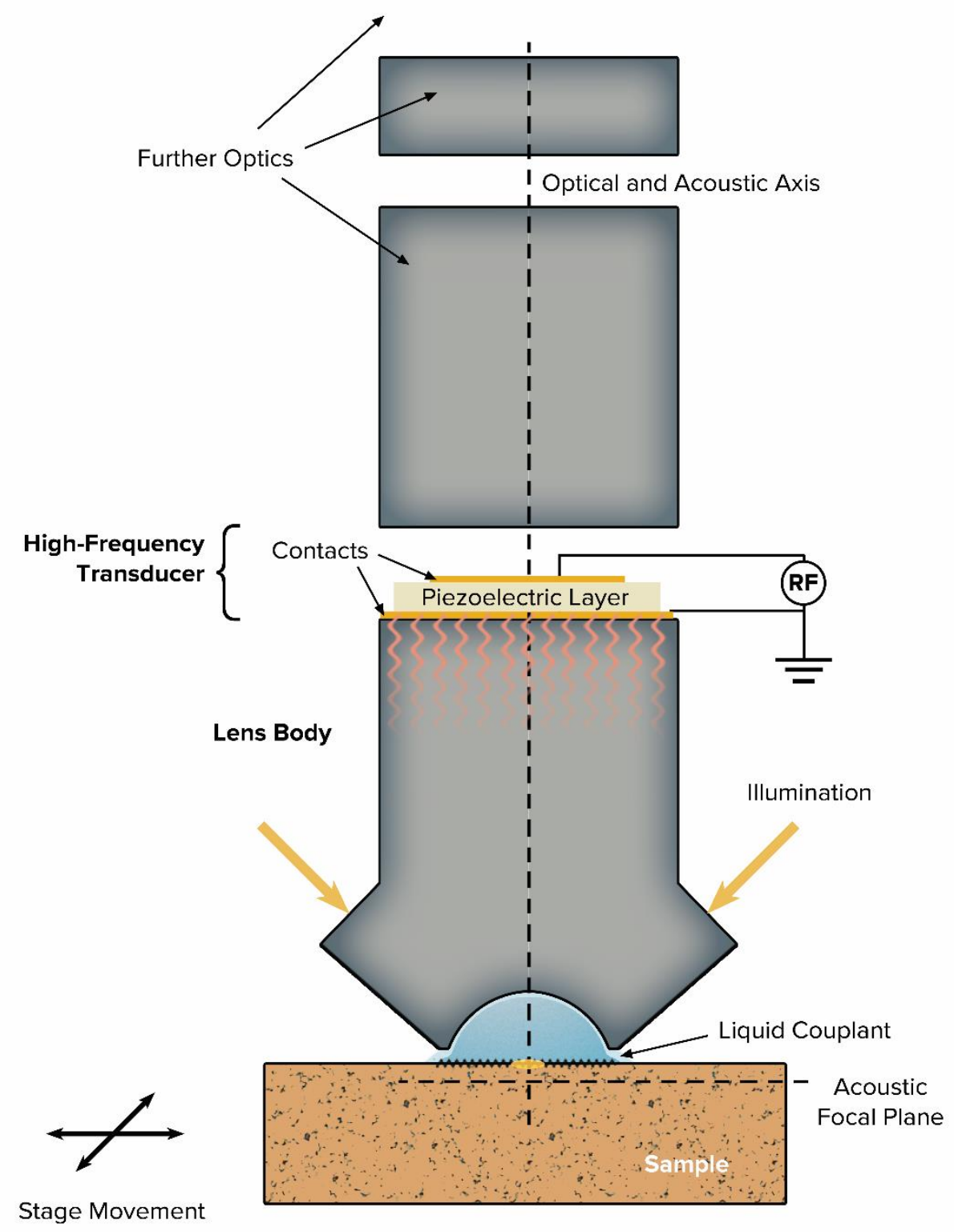

Figure 10.1: Illustration of the proposed acoustic-optical lens

The dual functionality $[33,34,35]$ of such a microscope is highly useful for sample characterization, as many NDE and medical related characterization techniques already rely on the combination of both imaging modalities, using separate acoustic and optical microscopes [10]. Given such a versatility, the resulting acoustic microscope would not require a scanning ability to establish a raster image of the sample; instead, the optical microscope could be used to 
quickly navigate the examined samples, while the acoustic microscope would only be used to image the areas of interest.

\subsection{Future Work}

I would put future work in the following categories:

- Piezoelectric measurements to quantify the piezoelectric activity of the film, and correlate these measurements to the developed microstructures.

- Develop various transducer structures and integrate them with the suitable acoustic lenses and matching layers.

- The development of an integrated optical-acoustical device as proposed above.

- Develop the suitable transparent coupling layers to maximize the acoustic matching between the sapphire faceplate and the investigated samples. 



\section{APPENDIX A}

\section{Appendix A: Ethics and Resourcing}

\subsection{Ethics}

There were no foreseeable ethics approval requirements for this study.

\subsection{Budget and Resources}

All the details related to the direct project costs were organized and provided by external project income. This covered lab materials and equipment operating costs. Useful textbooks and online literature materials were provided by the university library. A scholarship was provided through a subcontract with Callaghan Innovation. 



\section{APPENDIX B}

\section{Appendix B: Microfabrication Processes}

\subsection{Metal Contact Thermal Evaporation Recipe}

The following steps are followed during the deposition of the metal films:

1- The substrates are mounted onto the thermal evaporation holder

2- The correct stainless steel spacers and evaporation mask are mounted on top of the substrates using Philips screws.

3- Nitrogen gas is blown onto the holder's surface, to remove any debris from the exposed substrate surfaces.

4- The prepared sample holder is installed in the evaporation chamber.

5- The required evaporation sources are placed in the chamber.

6- The chamber is securely closed using hinges, then air is pumped out until the vacuum level reaches $2 \times 10^{-6}$ torr (usually overnight). 
7- The metal layers are evaporated at the fastest possible rates that ensure that the chamber pressure doesn't go above $1 \times 10^{-5}$ torr. For the bottom contact, a thin chromium layer $(0.5-2 \mathrm{~nm})$ is evaporated on top of the wafers, followed by the main contact material $(20-50 \mathrm{~nm}$ of gold). Then another chromium layer is deposited $(0.5-2 \mathrm{~nm})$. The top contact consists of a similarly thin (0.5-2nm) chromium layer, followed by the main contact material (20-50nm of silver or gold).

8- The chamber is vented into atmospheric pressure using nitrogen gas.

9- The sample holder is removed, and the sample is visually inspected, then prepared for the next processing step.

\subsection{Zinc Oxide Sputtering Recipe}

The following steps are followed to sputter zinc oxide films:

1- The substrates are mounted onto the sputtering holder. The same holder was used in the new mask design, to eliminate the need of substrate removal between thermal evaporation and sputtering steps. Also, the new mask design eliminated the need of using kapton tape for substrate placement.

2- The correct stainless steel spacers and sputtering mask are mounted on top of the substrates.

3- Nitrogen gas is blown onto the holder's surface, to remove any debris from the exposed substrate surface.

4- The required zinc oxide target is placed in the chamber. 
5- The prepared sample holder is installed in the sputtering chamber, at a constant sample-target distance, ranging from $51 \mathrm{~mm}$ to $92 \mathrm{~mm}$.

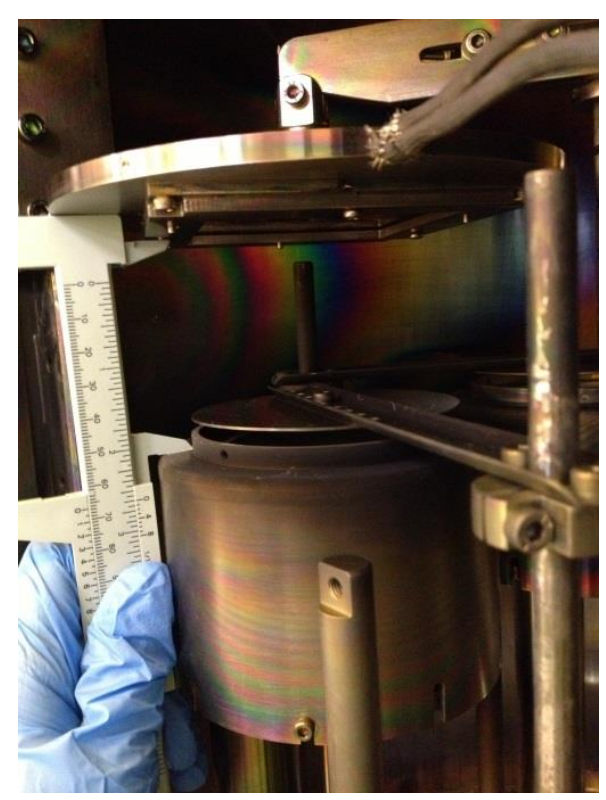

6- The necessary thermocouple and heating quartz lamp are installed in the chamber.

7- The chamber is securely closed using hinges, then air is pumped out until the vacuum level reaches a base pressure of at least $5 \times$ $10^{-6}$ mbar, which usually requires $4-5$ hours.

8- The sputtering and reactive gas flow rates are adjusted and the chamber is placed in the throttling mode, to allow the gases to flow through the high-vacuum valve. Typically, an argon gas flow rate of $6-8 \mathrm{sccm}$ is used for sputtering the buffer zinc oxide layer, where the high vacuum valve is manually adjusted to ensure having a sputtering chamber pressure of $1.5 \times 10^{-2}$ mbar. Then, the RF magnetron source is switched on, at the required power. This ranges from $150 \mathrm{~W}$ for the buffer layer, and up to $250 \mathrm{~W}$ for the rest of the 
film. During sputtering, the reflected power is monitored to ensure it staying below $2 \mathrm{~W}$.

9- Once the plasma has struck, the target is allowed to pre-sputter for $60 \mathrm{sec}$ to clean the target surface and get rid of any debris. Then the shutter is opened to start the deposition process.

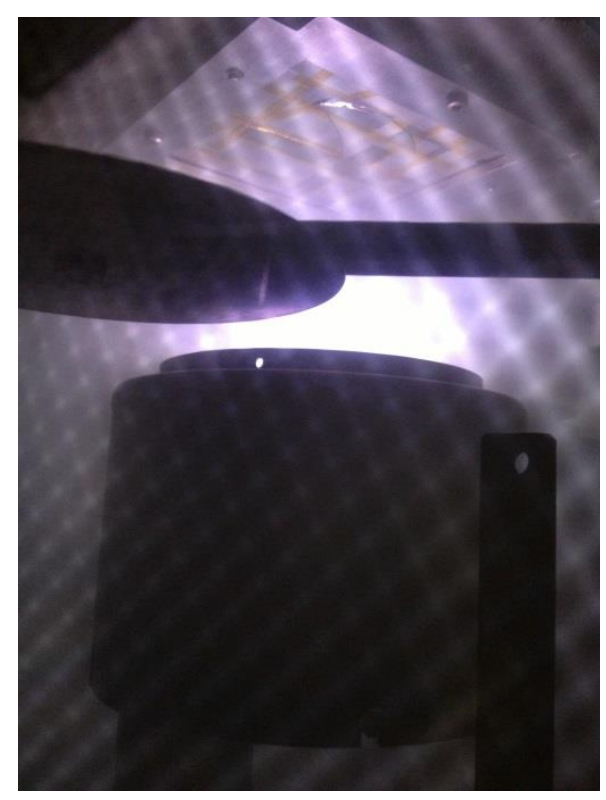

10 - The buffer layer is usually sputtered over $60 \mathrm{~min}$, during which the reflected RF power, substrate temperature, gas flow rate, and chamber pressure are monitored.

11-After the buffer layer is sputtered, the RF source is shut down, and the gas flow-rates are adjusted to prepare for the in situ annealing step. During that annealing step, a microcontroller is used to ramp the substrate temperature using a quartz lamp to the required level, which is limited to a maximum of $325^{\circ} \mathrm{C}$. Thereafter, substrate temperature is maintained for the required annealing time. After the annealing time has passed, the quartz lamp is turned off, and the substrates are allowed to cool to $40^{\circ} \mathrm{C}$. 
12- The sputtering and reactive gases are readjusted to prepare for the next zinc oxide film deposition. This layer is sputtered at a higher deposition rate, and sputtering usually lasts for several hours depending on the required film thickness.

13-Once sputtering is complete, the RF source and gases are shut down; then the chamber is vented into atmospheric pressure using nitrogen gas.

14- The sample holder is removed, and the sample is visually inspected, then prepared for the next processing step.

\subsection{Doped Zinc Oxide Sputtering Recipe}

The following steps are followed to sputter doped zinc oxide films:

1- The substrates are mounted onto the sputtering holder.

2- The correct stainless steel spacers and sputtering mask are mounted on top of the substrates.

3- Nitrogen gas is blown onto the holder's surface, to remove any debris from the exposed substrate surface.

4- The required doped zinc oxide target is placed in the chamber.

5- The prepared sample holder is installed in the sputtering chamber, at a constant sample-target distance, preferably at $92 \mathrm{~mm}$. The centre of the sample is moved 1 inch away from the target's central axis, to minimize the associated oxygen damage. 
6- The necessary thermocouple and heating quartz lamp are installed in the chamber.

7- The chamber is securely closed using hinges, then air is pumped out until the vacuum level reaches a base pressure of at least $5 \times$ $10^{-6}$ mbar, which usually requires 4-5 hours.

8- The sputtering and reactive gas flow rates are adjusted and the chamber is placed in the throttling mode, to allow the gases to flow through the high-vacuum valve. Typically, an argon gas flow rate of 9-12sccm is used for sputtering the doped zinc oxide layer, where the high vacuum valve is manually adjusted to ensure having a sputtering chamber pressure of $1.5 \times 10^{-3}$ mbar. The heating microcontroller is used to ramp the substrate temperature using a quartz lamp to the required level, which is limited to a maximum of $325^{\circ} \mathrm{C}$. Then, the RF magnetron source is switched on, at the required power. This ranges from $150 \mathrm{~W}$ to $200 \mathrm{~W}$. During sputtering, the reflected power is monitored to ensure it staying below $2 \mathrm{~W}$.

9- Once the plasma has struck, the target is allowed to pre-sputter for $60 \mathrm{sec}$ to clean the target surface and get rid of any debris. Then the shutter is opened to start the deposition process.

10-The doped zinc oxide layer is usually sputtered over $40-60 \mathrm{~min}$, during which the reflected RF power, substrate temperature, gas flow rate, and chamber pressure are monitored.

11-Once the required thickness is reached, the shutter is closed, and the $\mathrm{RF}$ power is shut down. Then, the chamber is allowed to cool down to room temperature. 
12- The chamber is vented into atmospheric pressure using nitrogen gas.

13- The sample holder is removed, and the sample is visually inspected, then prepared for the next processing step. 


\section{Bibliography}

[1] D. Ensminger and F. B. Stulen, Ultrasonics: Data, Equations, and their Practical Uses, CRC Press, Taylor and Francis Group, 2009.

[2] BCC Research, "Ultrasonics Technologies Markets MFG013F," BCC Research, 2016. [Online]. Available: http://www.bccresearch.com/marketresearch/manufacturing/ultrasonics-tech-market-mfg013f.html.

[3] G. Feng, C. Sharp, Q. Zhou, W. Pang, E. Kim and K. Shung, "Fabrication of mems ZnO dome-shaped-diaphragm transducers for high-frequency ultrasonic imaging," Journal of Microelectromechanics and Microengineering, vol. 15, p. 586, 2005.

[4] K. Shung, J. Cannata and Q. Zhou, "Piezoelectric materials for high frequency medical imaging applications: a review," Journal of ELectroceramics, vol. 19, pp. 139-145, 2007.

[5] A. Jakob, M. Bender, T. Knoll, R. Lemor, T. Lehnert, M. Koch, M. Veith, Q. Zhou, B. Zhu, J. Han and K. Shung, "Comparison of different piezoelectric materials for $\mathrm{GHz}$ acoustic microscopy transducers," IEEE International Ultrasoncis Symp. Proc., p. 1722, 2009.

[6] K. Shung and M. Zipparo, "Ultrasonic transducers and arrays," IEEE Eng. Med. Biol. Mag., vol. 15, p. 20, 1996.

[7] D. Turnbull, B. Starkoski, K. Harasiewicz, J. Semple, L. From, A. Gupta, D. Sauder and F. Foster, "A 40-100 MHz B-scan ultrasound backscatter microscope for skin imaging," Ultrasound in Medicine and Biology, vol. 21, p. 79, 1995.

[8] A.-J. Fresnel, "Mémoire sur la diffraction de la lumière," Annales de la Chemie et de Physique, vol. 1, no. 2, pp. 239-281, 1816.

[9] J. Cheeke, Fundamentals and Applications of Ultrasonic Waves, CRC Press, 2002. 
[10] K. Miura, H. Nasu and S. Yamamoto, "Scanning acoustic microscopy for characterization of neoplastic and inflammatory lesions of lymph nodes," Nature: Scientific Report 01255, 2013.

[11] Q. Zhou, S. Lau, D. Wu and K. K. Shung, "Piezoelectric films for high frequency ultrasonic transducers in biomedical applications," Progress in Material Science, no. 56, pp. 139-174, 2011.

[12] E. Klein, "Some Background History of Ultrasonics," The Journal of the Acoustical Society of America, vol. 20, no. 5, pp. 601-604, 1948.

[13] H. R. Atta, "High Frequency Ultrasound," British Journal of Ophthalmology, no. 79, pp. 967969, 1995.

[14] S. Manneville, L. Becu and A. Colin, "High-frequency ultrasonic speckle velocimetry in sheared complex fluids," The European Physical Journal, no. 28, pp. 361-373, 2004.

[15] Y. Goldberg, Properties of Advanced Semiconductor Materials GaN, AlN, InN, BN, SiC, SiGe, New York: John Wiley and Sons, 2001.

[16] D. Gerlich, S. Dole and G. Slack, J. Phys. Chem. Solids, vol. 47, no. 5, pp. 437-441, 1986.

[17] B. Aronov and R. Pasynkov, "The Great Soviet Encyclopedia, 3rd Edition," 1979. [Online]. Available: http://encyclopedia2.thefreedictionary.com/Piezoelectric+Materials.

[18] R. Lemons and C. Quate, "Acoustic microscope - scanning version," Appl. Phys. Lett., vol. 24, p. 163, 1974.

[19] I. Mina, H. Kim, I. Kim, S. Park, K. Choi, T. Jackson and R. Tutwiler, "High-frequency piezoelectric MEMs Ultrasound trasducers," IEEE Trans Ultrason Ferroelectr Freq Control, vol. 54, no. 12, p. 2422, 2007.

[20] F. Dauchy and R. Dorey, Journal Electroceram, vol. 19, p. 383, 2007.

[21] S. Lau, X. Li, Q. Zhou, K. Shung, J. Ryu and D. Park, "Aerosol-deposited KNN-LSO leadfree piezoelectric thick film for high frequency transducer applications," Sensors and Actuators A: Physical, vol. 163, p. 226, 2010.

[22] D.-M. Kivell, "Microstructural Control of ZnO Films Deposited by RF Magnetron 
Sputtering for Ultrasound Transducers," Victoria University of Wellington, 2012, 2012.

[23] M. Madou, Fundamentals of Microfabrication, Florida: CRC Press LLC, 2002.

[24] M. Ohring, Materials Science of Thin Films, San Diego: Academic Press, 2002.

[25] B. Su, "Novel Fabrication Processing for Improved Lead Zirconate Titanate (PZT) Ferroelectric Ceramic Materials," Facutly of Engineering of the University of Birmingham, PhD Thesis, 1998.

[26] D. Hahn, D. Park, J. Choi, W. Yoon, J. Ryu and D. Kim, "Effects of Zr/Ti ratio and postannealing temperature on the electrical properties of PZT thick films fabricated by aerosol deposition," Journal of Materials Research, vol. 23, no. 1, p. 226, 2008.

[27] IBMT Fraunhofer, [Online]. Available: http://www.ibmt.fraunhofer.de/en/Fields-ofwork/ibmt-ultrasound/ibmt-medical-ultrasound/ibmt-high-frequency-piezosystems.html. [Accessed 273 2014].

[28] E. P. Papadakis, "Fabrication and Characterization of Transducers," in Ultrasonic Instruments and Devices, Academic Press, 1999, p. 471.

[29] Imasonic, [Online]. Available: http://www.imasonic.com/Medical/IMHighFreq.php. [Accessed 273 2014].

[30] P. Muralt, "Piezoelectric films for innovations in the field of MEMS and biosensors," in Piezoelectricity: Evolution and Future of Technology, Berlin, Springer-Verlag, 2008, p. 351.

[31] U. Özgür, Y. I. Alivov, C. Liu, A. Teke, M. A. Reshchikov, S. Dogan, V. Avrutin, S.-J. Cho and $\mathrm{H}$. Morkoç, "A comprehensive review of $\mathrm{ZnO}$ materials and devices," Journal of Applied Physics, pp. 98, 041301, 2005.

[32] S. Pearton, D. Norton, K. Ip, Y. Heo and T. Steiner, "Recent progress in processing and properties of ZnO," Progress in Materials Science, no. 50, pp. 293-340, 2005.

[33] G. Thalhammer, R. Steiger, M. Meinschad, M. Hill, S. Bernet and M. Ritsch-Marte, "Combined acoustic and optical trapping," Biomed Opt Express, vol. 2, no. 10, p. 2859, 2011.

[34] C. R. P. Courtney, B. Drinkwater, C. C. S. Demore, A. Grinenko and P. Wilcox, "Dexterous manipulation of microparticles using Bessel-function acousitc pressure fields," Appl. Phys. 
Lett., vol. 102, 2013.

[35] L. Wang, "Multiscale photoacoustic microscopy and computed tomography," Nat Photonics, vol. 3, p. 503, 2009.

[36] P. Curie and P. J. Curie, "Comptes Rendus Hebdomadaires des Seances de l'Academie des Sciences," vol. Paris 91, p. 294, 1880.

[37] W. G. Cady, Piezoelectricity: An Introduction to the Theory and Applications of Electromechanical Phenomena in Crystals, New York: McGraw-Hill Book Company, Inc., 1946.

[38] W. Voigt, Lehrbuch der Kristallphysik, B.G. Teubner, 1910.

[39] W. Bragg and R. Gibbs, "The structure of alpha and beta quartz," Proc. R. Soc. Lond., vol. 109, pp. 405-427, 1925.

[40] A. Von Hippel, R. Breckenridge, F. Chesley and L. Tissa, Ind. Eng. Chem., no. 38, p. 1097, 1946.

[41] IEEE, "Standard definitions of terms associated with ferroelectric and related materials," IEEE Trans. Ultrason. Ferroelect. Freq. Cont., p. 1613, 2003.

[42] J. P. Joule, "On the effects of Magnetism upon the Dimensions of Iron and Steel Bars," Phil. Mag., vol. 3, no. 30, pp. 76-87, 1847.

[43] M. Ambersley and C. Pitt, "Piezoelectric $\mathrm{ZnO}$ transducers produced by rf magnetron sputtering," Thin solid films, vol. 80, p. 183, 1981.

[44] J. Galleo-Juarez, "Piezoelectric ceramic and ultrasound transducers," J. Phys. E: Sci. Instrum., vol. 22, p. 804, 1989.

[45] U. Ito, K. Kushida, K. Sugawara and H. Takeuchi, "A 100-MHz Ultrasonic Transducer Array Using ZnO Thin Films," IEEE Transactions on Ultrasonis, Ferroelectrics, and Frequency Control, vol. 42, no. 2, p. 316, 1995.

[46] Y. Hsu, J. Lin and A. Tang, "RF sputtered piezoelectric zinc oxide thin film for transducer applications," Journal of material science: materials in electronics, vol. 19, p. 653, 2007. 
[47] J. Tressler, S. Alkoy and R. Newnham, "Piezoelectric sensors and sensor materials," Journal of Electroceramics, vol. 2, p. 257, 1998.

[48] P. Muralt, R. Polcawich and S. Trolier-McKinstry, "Piezoelectric thin films for sensors, actuators, and energy harvesting," MRS Bulletin, vol. 34, no. 9, p. 658, 2009.

[49] W. Chang, Y. Chen, R. Lin, K. Cheng, K. Kao, B. Wu and Y. Huang, "Design and fabrication of piezoelectric transducer for wind-power generator," Thin solid films, vol. 519, p. $4687,2011$.

[50] G. Heckmann, "The lattice theory of solids," Ergeb. Exackt. Naturwiss., vol. 4, pp. 100-153, 1925.

[51] A. P. Dawson, "High Frequency Ultrasonic Wave Propagation in Anisotropic Materials," School of Chemical and Physical Sciences at Victoria University of Wellington, Wellington, 2010.

[52] S. Ramo, J. Whinnery and T. Van Duzer, Fields and Waves in Communication Electronics, New York: John Wiley and Sons, 1994.

[53] R. Newnham and L. Cross, "Ferroelectricity: the foundation of a field from form to funtion," MRS Bulletin, vol. 30, p. 845, 2005.

[54] W. Mason and R. Thurston, Physical Acoustics: Principles and Methods Vol. 15, 1981.

[55] W. Mason, "Sonics and Ultrasonics: Early History and Applications," IEEE Transactions on Sonics and Ultrasonics, Vols. SU-23, no. 4, p. 224, 1976.

[56] W. P. Mason, "Sonics and Ultrasonics: Early History and Applications," 1976 Ultrasonics Symposium, pp. 610-617, 1976.

[57] B. Vul and I. Goldman, C. R. Acad. Sci. USSR, no. 51, p. 21, 1946.

[58] J. D. N. Cheeke, "Ultrasonics: an Overview," in Fundamentals and Applications of Ultrasonic Waves, CRC Press, 2002.

[59] J. Pulskamp, R. Polcawich, R. Rudy, S. Bedair, R. Proie, T. Ivanov and G. Smith, "Piezoelectric PZT MEMS technologies for small-scale robotics and RF applications," MRS Bulletin, vol. 37, no. November, p. 1062, 2012. 
[60] Q. Su, P. Kirby, E. Komuro, M. Imura, Q. Zhang and R. Whatmore, "Thin-film bulk acoustic resonators and filters using $\mathrm{ZnO}$ and lead zirconium titanate thin films," IEEE Microwave Theory, vol. 49, p. 769, 2001.

[61] K. Lakin, "Thin film resonator technology," IEEE T Ultrason Ferr, vol. 52, p. 707, 2005.

[62] B. Jaffe, R. Roth and S. Marzullo, "Piezoelectric properties of Lead Zirconate-Lead Titanate solid-solution ceramics," Journal of Applied Physics, no. 25, p. 809, 1954.

[63] I. McColm, "Special Ceramics for Modern Applications: Which? Why? How?," in Ceramic Processing, Chapman and Hall, 1995, pp. 1-33.

[64] R. Newnham, "Molecular Mechanisms in Smart Materials," MRS Bulletin, vol. 22, pp. 2034, 1997.

[65] B. Jaffe, W. Cook Jr. and H. Jaffe, Piezoelectric Ceramics, New York: Academic Press, 1971.

[66] S. Swartz, T. Shrout and T. Takenaka, "Electronic Ceramics R\&D in the U.S., Japan," Am. Ceram. Soc. Bull., vol. 76, pp. 59-65, 1997.

[67] T. Shrout, "Innovations in piezoelectric materials for ultrasound transducers," Applications of Ferroelectrics, vol. 3, pp. 1-4, 2008.

[68] H. Chen, K. Udayakumar, C. Gaskey, L. Cross, J. Bernstein and L. Niles, "Fabrication and Electrical Properties of Lead Zirconate Titanate Thick Films," J. Am. Ceram. Soc., vol. 79, p. 2189, 1996.

[69] X. Hao, J. Zhai, F. Zhou, X. Song and S. An, "Thickness and frequency dependence of electric-field-induced strains of sol-gel derived (Pb0.97La0.02)(Zr0.95Ti0.05)O3 antiferroelectric films," Journnal of Sol-Gel Science and Technology, vol. 53, p. 366, 2010.

[70] B. Zhu, D. Wu, Q. Zhou, J. Shi and K. Shung, "Lead zirconate titanate thick film with enhanced electrical properties for high frequency transducer applications," Appl. Phys. Lett., vol. 93, p. 012905, 2008.

[71] X. Donglin, L. Meidong, Z. Yike, L. Churong, C. Shi., L. Shaobo and H. Yanqui, "Fabrication and electrical properties of lead zirconate titanate thick films using a new solgel processing technique," Journal of Materials Science: Materials in Electronics, vol. 12, p. 
$587,2001$.

[72] G. Haertling, "Intelligent Processing of Ferroelectric Films," AM. Ceram. Soc. Bull., vol. 73, p. 68,1994 .

[73] F. Lang, "Liquid Precursors for Ceramics: kinetically limited crystallisation, spherical particles, fibres, and thin films," in Chemical Processing of Advanced Materials, John Wiley and Sons, Inc., 1992, pp. 611-626.

[74] G. Yi and M. Sayer, "Sol-gel Processing of Complex Oxide Films," AM. Ceram. Soc. Bull., vol. 70, p. 1173, 1991.

[75] J. Akedo, "Room Temperature Impact Consolidation (RTIC) of Fine Ceramic Powder by Aerosol Deposition Method and Applications to Microdevices," Journal of Thermal Spray Technology, vol. 17, p. 181, 2008.

[76] M. O'Neill, "EU lead ban status (March 2004)," [Online]. Available: http://www.ami.ac.uk/courses/ami4982_lfi/u02/resources/Rohm_USA_3.pdf.

[77] EU Document, "Official Journal of the European Union," 17 2011. [Online]. Available: http://eur-lex.europa.eu/LexUriServ/LexUriServ.do?uri=OJ:L:2011:174:0088:0110:en:pdf.

[78] R. French, "Electronic Band Structure of Al2O3 With Comparison to AlON and AlN," Journal of American Ceramic Society, vol. 73, pp. 477-489, 1990.

[79] G. Slack, "Nonmetallic crystals with high thermal conductivity," Journal of Physical Chemical Solids, vol. 34, pp. 321-335, 1973.

[80] C. Eon and S. Trolier-McKinstry, "Thin-film piezoelectric MEMS," MRS Bulletin, vol. 37, no. November, p. 1007, 2012.

[81] G. Bu, D. Ciplys, M. Shur, L. Schowalter, S. Schujman and R. Gaska, "Surface acoustic wave velocity in single-crystal AIN substrates," IEEE Trans. Ultrason. Ferroelectr. Freq. Control, vol. 53, p. 251, 2006.

[82] P. Martin, M. Good, J. Johnston, G. Posakony, L. Bond and S. Crawford, "Piezoelectric films for 100-MHz ultrasonic transducers," Thin Solid Films, vol. 379, pp. 253-258, 2000.

[83] F. Martin, P. Muralt, M. Dubois and A. Pezous, "Thickness dependence of properties of 
highly c-axis textured AlN thin films," J. Vac. Sci. Tech. A, vol. 22, pp. 361-365, 2004.

[84] G. Piazza, V. Felmetsger, P. Muralt, R. Olsson III and R. Ruby, "Piezoelectric aluminum nitride thin films for microelectromechanical systems," MRS Bulletin, vol. 37, no. November, p. 1051, 2012.

[85] B. Bower, "Serbian site may have hosted first copper makers," 25 June 2010. [Online]. Available: https://www.sciencenews.org/article/serbian-site-may-have-hosted-firstcopper-makers.

[86] L. Schuler, "Ph.D. Thesis: Properties and Characterisation of Sputtered ZnO," University of Canterbury, Christchurch, 2008.

[87] P. Craddock, "Mining and Metallurgy," in The Oxford Handbook of Engineering and Technology in the Classical World, Oxford University Press, 2008, p. 111.

[88] Etacude, "Information on zinc oxide, philosopher's wool," etacude.com, 2014. [Online]. Available: http://chemicals.etacude.com/z/zinc_oxide.php.

[89] C. Jagadish and S. Pearton, Zinc Oxide Bulk, Thin Films and Nanosctructures, Elsevier, 2006.

[90] C. Bunn, "The lattice-dimensions of zinc oxide," P Phys Soc, vol. 47, pp. 835-842, 1935.

[91] E. Mollwo, "Z. Angew. Phys.," vol. 6, p. 257, 1954.

[92] A. Hutson, "Piezoelectricity and conductivity in $\mathrm{ZnO}$ and CdS," Physical Review Letters, vol. 4, no. 10, p. 505, 1960.

[93] S. Porto, B. Tell and T. Damen, "Near-Forward Raman Scattering in Zinc Oxide," Phys. Rev. Lett., vol. 16, pp. 450-452, 1966.

[94] F. Porter, Zinc Handbook: Properties, Processing, and Use in Design, CRC Press, 1991.

[95] K. Ueda, H. Tabata and T. Kawai, "Magnetic and electric properties of transition-metaldoped ZnO films," Appl. Phys. Lett., vol. 79, pp. 988-990, 2001.

[96] K. Kim, H. Kim, D. Hwang, J. Lim and S. Park, "Realization of p-type ZnO films via phosphorus doping and thermal activation of the dopant," Appl. Phys. Lett., vol. 83, pp. 63- 
65, 2003.

[97] H. Morkoç and U. Özgür, Zinc Oxide: Fundamentals, Materials and Device Technology, Weinheim: Wiley VCH Verlag GmbH, 2009.

[98] D. Thomas, "The exciton spectrum of zinc oxide," Journal of Physics and Chemistry of Solids, vol. 15, p. 86, 1960.

[99] D. Look, D. Reynolds, J. Hemsky, R. Jones and J. Sizelove, "Production and annealing of electron irradiation damage in ZnO," Appl. Phys. Lett., vol. 75, p. 811, 1999.

[100] A. Polyakov, N. Smirnov, A. Govorkov, E. Kozhukhova, V. Vdovin, K. Ip, M. Overberg, Y. Heo, D. Norton, J. Pearton, M. Zavada and V. Dravin, "Proton implantation effects on electrical and recombination propreties of undoped ZnO," J. Appl. Phys., vol. 94, p. 2895, 2003.

[101] M. Kadota, T. Miura and M. Minakata, "Piezoelectric and optical properties of ZnO films deposited by an electron-cyclotron-resonance sputtering system," J. Cryst. Growth, pp. 523$527,2002$.

[102] D. Look, "Recent advances in $\mathrm{ZnO}$ materials and devices," Materials Science and Engineering: B, vol. 80, pp. 383-387, 2001.

[103] I. Jeong, J. Kim and S. Im, "Ultraviolet-enhanced photodiode employing n-ZnO/ p-Si structure," Appl. Phys. Lett., vol. 83, p. 2946, 2003.

[104] A. Cheng, "One Dimensional Zinc Oxide Nanostructures for Optoelectronic Applications: Solar Cells and Photodiodes," Dissertation at the Electrical and Computer Engineering Department at Auburn University, 2008.

[105] Z. Wang, "Novel nanostructures of $\mathrm{ZnO}$ for nanoscale photonics, optoelectronics, piezoelectricity, and sensing," Materials Science and Processing, vol. 88, 2007.

[106] J. Cannata, J. Williams, Q. Zhou, L. Sun, K. Shung, H. Yu and E. Kim, "Self-focused ZnO transducers for ultrasonic biomicroscopy," Journal of Applied Physics, vol. 103, p. 084109, 2008.

[107] W. Hu, Z. Liu, J. Sun, S. Zhu, Q. Xu, D. Feng and Z. Ji, "Optical properties of pulsed laser deposited ZnO thin films," Journal of Applied Physics and Chemistry of Solids, vol. 58, pp. 853- 
857, 1997.

[108] R. Triboulet and J. Perriere, "Epitaxial growth of ZnO films," Progress in Crystal Growth and Characterization Materials, vol. 47, pp. 65-138, 2003.

[109] W. Water and S. Chu, "Physical and structural properties of ZnO sputtered films," Material Letters, vol. 55, p. 67, 2002.

[110] B. Khuri-Yakub, J. Smits and T. Barbee, "Reactive Magnetron Sputtering of ZnO," Journal of Applied Physics, vol. 52, pp. 4772-4774, 1982.

[111] C. Klingshirm, A. Waag, A. Hoffmann and J. Geurts, "Zinc Oxide: from funamental properties towards novel applications," Springer Series in Materials Science, vol. 120, 2010.

[112] Z. Al-Ahmed, "First-principles determination of the chemical potentials and vacancy formation energy in lead titanate and barium titanate and structural, electrical, and electromechanical responses in wurtzite $\mathrm{ZnO}$ under in-plane strain," University of Arkansas, 2007.

[113] O. Dulub, L. Boatner and U. Diebold, "STM study of the geometric and electronic structure of ZnO surfaces," Surface Science, vol. 519, no. 3, pp. 201-217, 2002.

[114] F. K., Quartz buffer rod lens focused 50MHz transducer, Waltham, MA: Panametrics Inc., 1971.

[115] J. Cannata, "Development of a 35-MHz Piezo-Composite ULtrasound Array for Medical Imaging," IEEE Transactions on Ultrasonics, Ferroelectric, and Frequency Control, vol. 53, no. 1, p. 224, 2006.

[116] J. Ketterling, O. Aristizabal, T. D.H. and F. Lizzie, IEEE Trans Ultrason Ferroelect Freq Contr, vol. 52, p. 672, 2005.

[117] R. Schwartz, "Chemical solution deposition of perovskite thin films," Chem Mater, vol. 9, p. $2325,1997$.

[118] D. Barrow, T. Petroff, P. Tandon and M. Sayer, J Appl Phys, vol. 81, p. 876, 1997.

[119] D. Barrow, T. Petroff and M. Sayer, Surf Coat Technol, vol. 113, no. 8, p. 76, 1995.

[120] R. Dorey and R. Whatmore, J Eur Ceram Soc, vol. 24, p. 1091, 2004. 
[121] J. Akedo and M. Lebedev, Appl Phys Lett, vol. 17101712, p. 77, 2000.

[122] K. Kajiyoshi, T. Ishizawa and Yoshumura, Jpn J Appl Phys, p. 30, 1990.

[123] Q. Zhang, F. Djuth, Q. Zhou, C. Hu, J. Cha and K. Shung, Ultrasonics, vol. 44, p. 711, 2006.

[124] J. Ryu, J. H. D. Choi, D. Park, W. Yoon and K. Kim, Appl Phys Lett, vol. 152901, p. 90, 2007.

[125] M. Lukacs, M. Sayer and F. Foster, "Single-element and linear array transducer design for ultrasound biomicroscopy," Proc. Med. Imag.: Ultrason. Transd. Eng., vol. 3341, p. 272, 1998.

[126] S. Michau, P. Mauchamp and R. Dufait, "Piezocomposite 30MHz linear array for medical imaging: design challanges and performances evaluation of a 128 element array," Proc IEEE Ultraon Symp, p. 898, 2004.

[127] D. Wu, Q. Zhou, X. L. C. Geng, F. Djuth and K. Shung, IEEE Trans Ultrason Ferroelectr Freq Contr, vol. 25, p. 2304, 2009.

[128] A. Dann, D. Bennett, R. Sing, J. Lemaire and W. Grundfest, Proc SPIE, p. 7629, 2010.

[129] M. Pan, T. Wu, T. Bui and W. Shih, "Fabrication of highly c-axis textured ZnO thin Tilms piezoelectric transducers by RF sputtering," Journal of Materials Science: Materials in Electronics, vol. 23, no. 2, p. 418, 2012.

[130] J. Zhang, W. Xu, G. Han, J. Carlier, X. Ji, S. Chen and B. Xu, "Micromachined highfrequency $\mathrm{ZnO}$ ultrasonic linear arrays," Ultrasonics Symposium (IUS) IEEE, pp. 512-515, 2013.

[131] Q. Zhou, C. Sharp, J. Cannata, K. Shung, G. Feng and E. Kim, "Self-focused high frequency ultrasonic transducers based on $\mathrm{ZnO}$ piezoelectric films," Applied Physics Letters, vol. 90, no. 113502, 2007.

[132] H. Morkoc, Nitride Semiconductors and Devices, Berlin: Springer, 1999.

[133] P. Fons, K. Iwata, S. Niki, A. Yamada and K. Matsubara, "Growth of high-quality epitaxial ZnO films on alpha-Al2O3," J. Crystal Growth, vol. 201, pp. 627-632, 1999.

[134] Y. Chen, D. Bagnall, H. Koh, K. Park, K. Hiraga, Z. Zhu and T. Yao, "Plasma assisted molecular beam epitaxy of $\mathrm{ZnO}$ on c-plane sapphire: Growth and characterization," J. 
Appl. Phys., vol. 84, p. 3912, 1998.

[135] K.-K. Kim, J.-H. Song, H.-J. Jung, W.-K. Choi, S.-J. Park, J.-H. Song and J.-Y. Lee, "Photoluminescence and heteroepitaxy of $\mathrm{ZnO}$ on sapphire substrate (0001) grown by rf magnetron sputtering," Journal of Vacuum Science \& Technology A, vol. 18, no. 6, p. 2864, 2000.

[136] R. Vispute, V. Talyansky, S. Choopun, R. Sharma, T. Venkatesan, M. He, X. Tang, J. Halpern, M. Spencer, Y. Li, L. Salamanca-Riba and A. Iliadis, "Heteroepitaxy of ZnO on GaN and its implications for fabrication of hybrid optoelectronic devices," Appl. Phys. Lett., vol. 73, p. 348, 1998.

[137] M. Kasuga and S. Ogawa, "Electronic Properties of Vapor-Grown Heteroepitaxial ZnO Film on Sapphire," Jpn. J. Appl. Phys., vol. 22, pp. 794-798, 1983.

[138] N. Takahashi, K. Kaiya, T. Nakamura, Y. Momose and H. Yamamoto, "Growth of ZnO on Sapphire (0001) by the Vapor Phase Epitaxy Using a Chloride Source," Jpn. J. Appl. Phys., vol. 38, p. 454, 1999.

[139] Y. Liu, C. Gorla, S. Liang, N. Emanetoglu, Y. Lu, H. Shen and M. Wraback, "Ultraviolet detectors based on epitaxial ZnO films grown by MOCVD," Journal of Electronic Materials, vol. 29, no. 1, pp. 69-74, 2000.

[140] J. Vossen, Physics of Thin Films, Vol. 9, New York: Academic Press, 1977.

[141] D. Kang, J. Kim, S. Jeong, Y. Roh, S. Jeong and J. Boo, "Structural and electrical characteristics of R.F. magnetron sputtered ZnO films," Thin Solid Films, vol. 475, pp. 160165, 2005.

[142] R. Lad, P. Funkenbusch and C. Aita, "Postdeposition annealing behavior of rf sputtered ZnO films," J. Vac. Sci. Technol., vol. 17, p. 808, 1980.

[143] A. Anders, "A structure zone diagram including plasma-based deposition and ion etching," Thin Solid Films, vol. 518, no. 15, pp. 4087-4090, 2010.

[144] B. Movchan and A. Demchishin, "Study of the Structure and Properties of Thick Vacuum Condensates of Nickel, Titanium, Tungsten, Aluminum Oxide, and Zirconium Dioxide," Fizika Metallov i Metallovedenie (English Translation), vol. 28, pp. 83-90, 1969. 
[145] J. Thornton, "Influence of apparatus geometry and deposition conditions on the structure and topography of thick sputtered coatings," J. Vac. Sci. Technol., vol. 11, p. 666, 1974.

[146] L. Freund and S. Suresh, Thin Film Materials: Stress, Defect Formation and Surface Evolution, Cambridge: University Press, 2003.

[147] S. Campbell, The science and engineering of microelectronic fabrication, Oxford: University Press, 2001.

[148] A. Cimpoiasu, N. Van der Pers, T. deKeyser, A. Venema and M. Vellekoop, "Stress control of piezoelectric ZnO films on silicon substrates," Smart Materials and Structures, vol. 5, pp. 744-750, 1996.

[149] W. King, "Piezoelectric Sorption Detector," Anal. Chem., vol. 36, no. 9, pp. 1735-1739, 1964.

[150] B. Wacogne, M. Roe, T. Pattinson and C. Pannell, "Effective piezoelectric activity of zinc oxide films grown by radio frequency planar magnetron sputtering," J. Appl. Phys., vol. 67, 1995.

[151] S. Rossnagel, "Sputtering and Sputter Deposition," in Handbook of Thin Film Deposition Processes and Techniques, Norwich, Noyes Publications, 2002, pp. 319-348.

[152] B. Aufderheide, "Coating and Processing Techniques," in Coatings Technology Handbook, Third Edition, CRC Press, 2005, pp. 30-1.

[153] T. Miysoshi, Jpn J Appl Phys, vol. 46, p. 7023, 2007.

[154] R. Hong, J. Huang, H. He and Z. S. J. Fan, "Influence of different post-treatments on the structure and optical properties of zinc oxide films"," Applied Surface Science, vol. 242, p. 346, 2005.

[155] Veeco, "Dektak 150 Surface Profiler," 2009. [Online]. Available: http://www.upc.edu/pct/documents_equipament/d_81_id-399.pdf.

[156] B. Cullity and S. Stock, "Properties of X-rays," in Elements of X-ray Diffraction, New Jersey, Prentice Hall, 2001, pp. 1-28.

[157] W. Roentgen, Uber Eine Neue Art Von Strahlen, Wurzburg Stahel, 1896. 
[158] R. A. Novelline and L. F. Squire, Squire's Fundamentals of Radiology, Harvard University Press, 2004.

[159] M. Eckert, "Disputed discovery: the beginnings of X-ray diffraction in crystals in 1912 and its repercussions," Acta Crystallographica: Foundations of Crystallography, no. A68, pp. 30-39, 2012.

[160] A. H. Compton, "A Quantum Theory of the Scattering of X-rays by Light Elements," Phys. Rev., no. 21, pp. 483-502, 1923.

[161] D. E. Billings, A guide to the solar corona, New York: Academic Press, 1966.

[162] W. L. Bragg, "The diffraction of short electromagnetic waves by a crystal," Proc. Cambridge Phil. Soc., vol. 17, pp. 43-57, 1913.

[163] B. Cullity and S. Stock, "Diffraction III: Real Samples," in Elements of X-Ray Diffraction, New Jersey, Prentice Hall, 2001, pp. 167-184.

[164] B. Cullity and S. Stock, "Diffractometer Measurement," in Elements of X-Ray Diffraction, New Jersey, Prentice Hall, 2001, p. 186.

[165] B. Cullity and S. Stock, "Diffraction II: Intensities of Diffracted beams," in Elements of X-ray Diffraction, New Jersey, Prentice Hall Inc., 2001, p. 123.

[166] PANalytical, "PANalytical-X'Pert Powder," PANalytical, [Online]. Available: http://www.panalytical.com/XPert-Powder.htm.

[167] B. Cullity and S. Stock, "Precise Parameter Measurements," in Elements of X-Ray Diffraction, New Jersey, Prentice Hall, 2001, p. 367.

[168] S. A. Speakman, "Basics of X-Ray Powder Diffraction," MIT, [Online]. Available: http://prism.mit.edu/xray/1\%20Basics\%20of\%20X-Ray\%20Powder\%20Diffraction.pdf.

[169] B. Cullity and S. Stock, "Appendix 3," in Elements of X-ray Diffraction, New Jersey, Prentice Hall, 2001, p. 633.

[170] ZnO Powder, Calculated from ICSD using POWD-12++, Reference code: 01-070-8072, 2001.

[171] M. Chen, Z. Pie, X. Wang, C. Sun and L. Wen, "Dependence of structural, electrical, and optical properties of $\mathrm{ZnO}$ films on substrate temperature," Journal of Materials Research, vol. 
16, p. 2118, 2001.

[172] A. Golubovic, S. Nikolic, S. Djuric and A. Valcic, "The growth of sapphire single crystals," J. Sehem. Soc., vol. 66, no. 6, pp. 411-418, 2001.

[173] A. L. Patterson, "The Scherrer Formula for X-ray Particle size Determination," Phys. Rev., no. 56, pp. 978-982, 1939.

[174] P. Scherrer, "Bestimmung der Groesse und der inneren Struktur von Kolloidteilchen mittels Roentgenstrahlen," Nachr. Ges. Wiss. Goettingen, no. 26, pp. 98-100, 1918.

[175] J. Langford and A. Wilson, "Sherrer after Sixty Years: A Survey and Some New Results in the Determination of Crystallite Size," J. Appl. Cryst., no. 11, pp. 102-113, 1978.

[176] Agilent Technologies, Agilent 4156C Precision Semiconductor Parameter Analyzer, User's Guide, Palo Alto: Agilent Technologies, Inc., 2005.

[177] L. van der Pauw, "A method of measuring the resistivity and hall coefficient on lamellae of arbitrary shape," Philips Technical Review, vol. 20, p. 220, 1958.

[178] NIST, "Physical Measurement Laboratory," NIST, April 2010. [Online]. Available: http://www.nist.gov/pml/div683/hall_algorithm.cfm. [Accessed 06 2016].

[179] J. Goldstein, D. Newbury, D. Joy, C. Lyman, P. Echlin, E. Lifshin, L. Sawyer and J. Michael, Scanning Electron Microscopy and X-ray Microanalysis, 3rd Edition, New York: Plenum Publishers, 2003.

[180] J. Manifacier, J. Gasiot and J. Fillard, "A simple method for the determination of the optical constants $\mathrm{n}, \mathrm{k}$ and the thickness of a weakly absorbing film," J. Phs. E: Sci. Instrum., vol. 9, p. $1002,1976$.

[181] R. Swanepoel, "Determination of the thickness and optical constants of amorphous silicon," J. Phys. E: Sci. Instrum., vol. 16, p. 1214, 1983.

[182] I. Chambouleyron, J. Martinez, A. Moretti and M. Mulato, "Retrieval of optical constants and thickness of thin films from transmission spectra," Applied Optics, vol. 36, no. 31, p. 8238, 1997.

[183] G. Gorachand, E. Michiyuki and I. Takashi, "Temperature-dependent Sellmeier 
Coefficients and chromatic dispersions for some optical fiber glasses," Journal of Lightwave Technology, vol. 12, no. 8, p. 1338, 1994.

[184] X. Sun and H. Kwok, "Optical properties of epitaxially grown zinc oxide films on sapphire by pulsed laser deposition," Journal of Applied Physics, vol. 86, no. 1, p. 408, 1999.

[185] J. Tauc, R. Grigorovici and A. Vancu, "Optical Properties and Electronic Structure of Amorphous Germanium," Phys. Stat. Sol., vol. 15, p. 627, 1966.

[186] T. McLean, "A.F. Gibson (Ed.), Progress in Semiconductors, Vol. 5," London, Heywood and Co. Ltd., 1960, pp. 55-102.

[187] E. Davis and N. Mott, "Conduction in non-crystalline systems V: conductivity, optical absorption and photoconductivity in amorphous semiconductors," Philosophical Magazine, vol. 22 , no. 179 , p. $903,1970$.

[188] B. Grolik and J. Kopp, "Optical Properties of Thin Semiconductor Films," 31102003. [Online]. Available: http://home.fnal.gov/ jkopp/tum/pdf/F/hl_spekt.pdf. [Accessed 2006 2016].

[189] R. Singh, M. Kumar and S. Chandra, "Growth and characterization of high resistivity caxis oriented $\mathrm{ZnO}$ films on different substrates by RF magnetron sputtering for MEMS applications," Journal of Materials Science, vol. 42, no. 12, pp. 4675-4683, 2007.

[190] A. Anderson and D. Oates, "RF magnetron sputtering of ZnO for SAW: effects of magnetic field strength and configuration," Ultrasonics Symposium, p. 329, 1982.

[191] K. Tominaga, K. Kuroda and O. Tada, "Radiation effect due to energetic oxygen atoms on conductive Al-doped ZnO films," Japanese Journal of Applied Physics, vol. 27, no. 7, pp. 11761180, 1988.

[192] E. Yong, B. Jae, J. Young, K. Hyung, C. Jong and J. Hyeong, "Microstructural evolution and preferred orientation change of radio-frequency-magnetron sputtered $\mathrm{ZnO}$ thin films," $J$. Vac. Sci. Technol. A., vol. 14, no. 3, p. 1943, 1996.

[193] J. Molarius, J. Kaitila, T. Pensala and M. Ylilammi, "Piezoelectric ZnO films by rf sputtering," Journal of Materials Science: Materials in Electronics, vol. 14, p. 431, 2003. 
[194] S. Cho, "Effects of growth temperature on the properties of $\mathrm{ZnO}$ thin films grown by radio-frequency magnetron sputtering," Transactions on Electrical and Electronic Materials, vol. 10, p. 185, 2009.

[195] S. Singh, R. Srinivasa and S. Major, "Effect of substrate temperature on the structure and optical properties of $\mathrm{ZnO}$ thin films deposited by reactive rf magnetron sputtering," Thin Solid Films, vol. 515, p. 8718, 2007.

[196] S. Lin, J. Huang and D. Luii, "The effects of RF power and substrate temperature on the properties of ZnO films," Surf Coat Tech, vol. 176, p. 179, 2003.

[197] J. H. Jou, M. Y. Han and D. J. Chen, "Substrate dependent internal stress in sputtered zinc oxide thin films," Journal of Applied Physics, vol. 71, p. 4333, 1992.

[198] R. Cebulla, R. Wendt and K. Ellmer, "Al-doped zinc oxide films deposited by simultaneous rf and dc excitation of magnetron plasma: Relationships between plasma parameters and structural and electrical properties," Journal of Applied Physics, vol. 83, p. 1087, 1998.

[199] H. Windischmann, "Intrinsic stress in sputtered thin films," Journal of Vacuum Science Technology A: Vacuum, Surfaces, vol. 9, p. 2431, 1991.

[200] R. Kock, "The intrinsic stress of polycrystalline and epitaxial thin metal films," Journal of Physics: Condensed Matter, vol. 6, p. 9519, 1994.

[201] K. Muller, "Stress and microstructure of sputter deposited thin films: Molecular dynamics investigations," Journal of Applied Physics, vol. 62, p. 1796, 1987.

[202] W. Nix, "Mechanical properties of thin films," Metallurgical and materials transactions, vol. 20, p. 2217, 1989.

[203] J. D. Rancourt, Optical thin films: user handbook, Washington, USA: SPIE Press, 1996.

[204] L. Garcia-Gancedo, J. Pedros, Z. Zhu, A. Flewitt and W. Milne, "Room-temperature remote-plasma sputtering of c-axis oriented zinc oxide thin films," Journal of Applied Physics, vol. 112, p. 014907, 2012.

[205] Y. E. Lee, J. Lee, Y. Kim, H. Yang, J. Park and H. Kim, "Microstructural evoluion and preferred orientation change of radio-frequency-magnetron sputtered $\mathrm{ZnO}$ thin films," 
American Vacuum Society, vol. 14, no. 3, pp. 1943-1948, 1996.

[206] L. Chun-Cheng, C. Chia-Chiang, W. Chin-Jyi, T. Zong-Liang, T. Jian-Fu, C. Sheng-Yuan, C. Yi-Chun and Q. Xiaoding, "In-situ post-annealing technique for improving piezoelectricity and ferroelectricity of $\mathrm{Li}$-doped $\mathrm{ZnO}$ thin films prepared by radio frequency magnetron sputtering system," Applied Physics Letters, vol. 102, p. 102107, 2013.

[207] L. Yu Tong, G. Zhiqang, Q. Wei Wei, W. Qiu Jun, J. Ma Xian, W. D., C. Xiaoqiang, F. Hu Xue and Z. Wei, "Nano Size Related Piezoelectric Efficiency in a Large ZnO Thin Film, Potential for Self-Powered Medical Device Application," Biochemistry and Analytical Biochemistry, vol. 5, no. 1, p. 1000243, 2016.

[208] A. Aarti, A. Anil, Dwivedi, V.K., P. George and G. Vinay, "Effect of post deposition annealing temperature on the properties of $\mathrm{ZnO}$ films prepared by $\mathrm{RF}$ magnetron sputtering," Physics of Semiconductor Devices, p. 500, 2007.

[209] HHV Ltd., Auto 500 RF Sputter Coater: Instruction Manual, West Sussex, 2006.

[210] J. Mizsei and V. Lanto, "In situ AFM, XRD, and resistivity studies of the agglomeration of sputtered silver nanolayers," Journal of Nanoparticle Research, vol. 3, p. 271, 2001.

[211] C. M. Muller and R. Spolenak, "Microstructure evolution during dewetting in thin Au films," Acta Materialia, vol. 58, p. 6035, 2010.

[212] H. Sadan and W. Kaplan, "Au-Sapphire (0001) solid-solid interfacial energy," Journal of Material Science, vol. 41, p. 5099, 2006.

[213] D. Danielson, D. Sparacin, J. Michel and L. Kimerling, "Surface-energy-driven dewetting theory of SOI agglomeration," Journal of Applied Physics, vol. 100, p. 083507, 2006.

[214] C. Thompson, "Solid-state dewetting of thin films," Annu. Rev. Mater. Res., vol. 42, p. 399, 2012.

[215] W. P. G.A. Rozgonyi, "Preparation of ZnO Thin Films by Sputtering of the Compound in Oxygen and Argon," Applied Physics Letters, vol. 8, no. 9, p. 220, 1966.

[216] G. L. K.S. Chou, "Effects of Excess Zinc on the Structure of Reactively Sputtered ZincOxide Films," Materials Chemistry and Physics, vol. 37, no. 2, pp. 156-160, 194. 
[217] K. Kanaya and S. Okayama, "Penetration and energy-loss theory of electrons in solid targets," Journal of Physics D: Applied Physics, vol. 5, p. 43, 1972.

[218] J. Kwon, T. Yoon, K. Kim and S. Min, "Comparison of the agglomeration behavior of Au and $\mathrm{Cu}$ films sputter deposited on silicon dioxide," Jounral of Applied Physics, vol. 93, p. 3270, 2003.

[219] H. Moseley, "The high-frequency spectra of the elements, Part 1," Philosophical Mag, vol. 26, 1913.

[220] C. Muller and R. Spolenak, "Microstructure evolution during dewetting in thin Au films," Acta Materialia, vol. 58, p. 6035, 2010.

[221] R. Belser and W. Hicklin, "Temperature coefficients of resistance of metallic films in the temperature range $25^{\circ}$ to $600^{\circ} \mathrm{C}$," Journal of Applied Physics, vol. 30, p. 313, 1959.

[222] C. Pennetta, L. Reggiani and G. Trefan, "Scaling and universality in electrical failure of thin films," Physical Review Letters, vol. 84, p. 5006, 2000.

[223] K. Sieradzki, K. Bailey and T. Alford, "Agglomeration and percolation conductivity," Applied Physics Letters, vol. 79, p. 3401, 2001.

[224] A. Mayadas and M. Shatzkes, "Electrical-resistivity model for polycrystalline films: the case of arbitrary reflection at external surfaces," Phys Rev B, vol. 1, p. 1382, 1970.

[225] L. Brillson and Y. Lu, "ZnO Schottky barriers and Ohmic contacts," Applied Physics Reviews, vol. 109, p. 121301, 2011.

[226] P. Gondoni, M. Ghidelli, F. Di Fonzo, M. Carminati, V. Russo, A. Li Bassi and C. Casari, "Structure-dependent optical and electrical transport properties of nanostructured Aldoped ZnO," Nanotechnology, vol. 23, p. 365706, 2012.

[227] T. Minami, "Transparent conducting oxide semiconductors for transparent electrodes," Semicond. Scie. Technol., vol. 20, p. S35, 2005.

[228] K. Ellmer, "Magnetron Sputtering of transparent conductive zinc oxide: relation between the sputtering parameters and the electronic properties," J. Phys. D. Appl. Phys., vol. 33, p. $17,2000$. 
[229] T. Hada, K. Wasa and S. Hayakawa, "Structures and electrical properties of zinc oxide films by low pressure sputtering system," Thin Solid Films, vol. 7, p. 135, 1970.

[230] T. Minami, H. Sato, H. Imamoto and S. Takata, "Substrate temperature dependence of trasparent conducting $\mathrm{Al}$-doped $\mathrm{ZnO}$ thin films perpared by magnetron sputtering," Jpn. J. Appl. Phys., vol. 31, p. 257, 1992.

[231] T. Nakazawa and K. Ito, J. Vac. Soc. Jpn., vol. 26, p. 889, 1983.

[232] T. Welzel, R. Kleinhempel, T. Dunger and F. Richter, "Ion Energy Distributions in Magnetron Sputtering of Zinc Aluminum Oxide," in PSE Proceedings, 2009.

[233] M. Marinov and D. Dobrev, Thin Solid Films, vol. 42, p. 265, 1977.

[234] R. Kaltofen and G. Weise, J. Nuclear Mater., vol. 200, p. 375, 1993.

[235] J. Harper, J. Cuomo and H. Hentzell, Apply. Phys. Lett., vol. 43, p. 547, 1983.

[236] G. W. J. Brodie, Y. Qiu, S. Cochran, G. C. Spalding and M. MacDonald, "Optically Transparent Piezoelectric Transducer for Ultrasonic Particle Manipulation," IEEE Transactions on Ultrasonics, Ferroelectrics, and Frequency Control, vol. 61, no. 3, p. 389, 2014. 